\title{
Coral calcification: insights from inorganic experiments and coral responses to environmental variables
}

\author{
By \\ Michael Holcomb \\ B.S., University of Idaho, 2004 \\ Submitted in partial fulfillment of the requirements for the degree of \\ Doctor of Philosophy \\ at the \\ MASSACHUSETTS INSTITUTE OF TECHNOLOGY \\ and the \\ WOODS HOLE OCEANOGRAPHIC INSTITUTION
}

February 2010

(c) 2010 Michael Holcomb. All rights reserved.

The author hereby grants MIT and WHOI permission to reproduce and to distribute publicly paper and electronic copies of this thesis document in whole or in part in any medium now known or hereafter created.

Author.

Joint Program in Oceanography/Applied Ocean Science and Engineering Department of Geology and Geophysics Massachusetts Institute of Technology and Woods Hole Oceanographic Institution December 7, 2009

Certified by

Anne L. Cohen

Research Specialist, Department of Geology and Geophysics, WHOI

Thesis Co-Supervisor

Certified by

Daniel C. McCorkle

Associate Scientist, Department of Geology and Geophysics, WHOI

Thesis Co-Supervisor

Accepted by

Bradford H. Hager

Professor of Earth, Atmospheric, and Planetary Sciences, MIT

Chairman, Joint Committee for Geology and Geophysics 


\section{Table of Contents}

Abstract...................................................................... 5

Acknowledgements...................................................... 7

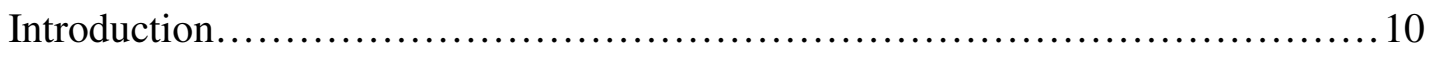

Chapter 1. Compositional and morphological features of aragonite precipitated experimentally from seawater and biogenically by corals ......... 16

Chapter 2. Timing of daily growth band formation in coral skeletons ....... 62

Chapter 3. Long-term effects of nutrient and $\mathrm{CO}_{2}$ enrichment on the temperate coral Astrangia poculata (Ellis and Solander, 1786)............. 103

Chapter 4. Coral gender: an unexplored factor in coral calcification? ........ 138

Synthesis...................................................... 168

\section{Appendices}

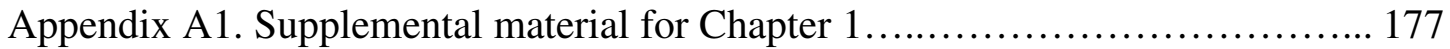

Appendix A2. Supplemental experiments for Chapter $2 \ldots \ldots \ldots \ldots \ldots \ldots \ldots \ldots \ldots . \ldots 6$

Appendix A3. Additional data for Chapter 3............................ 213

Appendix A4. Additional data for Chapter 4............................. 215

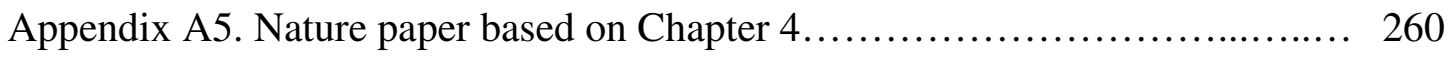

Appendix A6. Supplemental material for Appendix 5........................ 266 


\title{
Coral calcification: insights from inorganic experiments and coral responses to environmental variables
}

By

\author{
Michael Holcomb
}

Submitted to the MIT/WHOI Joint Program in Oceanography/Applied Ocean Science and Engineering on November 18, 2009 in partial fulfillment of the requirements for the degree of Doctor of Philosophy in Marine Geology

\begin{abstract}
The mechanisms involved in the formation of coral skeletons are examined using a laboratory model for coral calcification and the growth of living corals under different environmental conditions. Abiogenic aragonite was precipitated from seawater over a range of saturation states (Chapter 1). Abiogenic aragonite formed at high saturation state $(\Omega>\sim 20)$ had a granular appearance and was enriched in trace elements, similar to the crystals found within the centers of calcification and dark bands in coral skeletons. Abiogenic aragonite formed fibrous crystals at lower saturation states, similar to the crystals which radiate out from the centers of calcification. These similarities suggest that the internal calcifying environment of the coral experiences a wide range of saturation states. To estimate when periods of high or low saturation state occur within the coral, corals were stained to mark the skeleton deposited during specific time intervals (Chapter 2). Dark bands are shown to form between dusk and dawn. A conceptual model is proposed in which daytime saturation state is limited by the availability of $\mathrm{CO}_{2}$ due to the uptake of $\mathrm{CO}_{2}$ by photosynthesis. To test the potential for photosynthesis to limit $\mathrm{CO}_{2}$ availability to calcification, corals were grown under experimentally manipulated $\mathrm{CO}_{2}$ and nutrient levels. Elevated $\mathrm{CO}_{2}$ levels were found to decrease calcification in zooxanthellate colonies of the coral Astrangia poculata, however the addition of inorganic nutrients reduced the negative impact of $\mathrm{CO}_{2}(\mathrm{Chapter}$ 3 ), while reduced calcification rates were associated with elevated nutrients at ambient $\mathrm{CO}_{2}$ levels. Together these results suggest that nutrient availability may limit photosynthesis under elevated $\mathrm{pCO}_{2}$ conditions, while at ambient conditions additional stimulation of photosynthesis may limit the $\mathrm{CO}_{2}$ supply to calcification. To further test the interaction of photosynthesis with calcification, the effects of nutrients, $\mathrm{CO}_{2}$, and temperature were tested on both zooxanthellate and azooxanthellate coral colonies (Chapter 4). No clear pattern of nutrient enhancement of photosynthesis or calcification was found. However, a pronounced gender difference was found in the effect of $\mathrm{CO}_{2}$ on calcification in spawning corals, with female corals being more sensitive to elevated $\mathrm{CO}_{2}$.
\end{abstract}




\begin{abstract}
Abbreviated abstract
Coral calcification is examined using a laboratory model and living corals. In the laboratory model, abiogenic aragonite formed at high saturation state $(\Omega>\sim 20)$ had a granular appearance and was enriched in trace elements, similar to centers of calcification and dark bands in corals. Abiogenic aragonite formed fibrous crystals at lower saturation states, similar to crystals which radiate out from centers of calcification. These similarities suggest the calcifying environment of the coral experiences a range of saturation states. To estimate when high or low saturation states occur within the coral, living corals were stained, staining patterns suggest dark bands form between dusk and dawn. A model is proposed in which daytime saturation state is limited by the availability of $\mathrm{CO}_{2}$. To test the potential for photosynthesis to limit $\mathrm{CO}_{2}$ availability to calcification, corals were grown under altered $\mathrm{CO}_{2}$ and nutrient levels. Elevated $\mathrm{CO}_{2}$ levels decreased calcification in zooxanthellate corals, however addition of nutrients reduced the negative impact of $\mathrm{CO}_{2}$. This suggests nutrient availability may limit photosynthesis under elevated $\mathrm{pCO}_{2}$ conditions. The effects of nutrients, $\mathrm{CO}_{2}$, and temperature were further tested on both zooxanthellate and azooxanthellate coral colonies. Unexpectedly, a gender difference was found in the effect of $\mathrm{CO}_{2}$ on calcification.
\end{abstract}

\title{
Thesis Supervisors:
}

Dr. Anne L. Cohen, Research Specialist, WHOI

Dr. Daniel C. McCorkle, Associate Scientist, WHOI

\section{Defense Chair:}

Dr. Laura Robinson, Assistant Scientist, WHOI

\section{Thesis Committee:}

Dr. Tanja Bosak, Assistant Professor, MIT

Dr. Glenn A. Gaetani, Associate Scientist, WHOI

Dr. Scott Gallager, Associate Scientist, WHOI

Dr. Ann M. Tarrant, Assistant Scientist, WHOI 


\section{Acknowledgements}

I must thank my family for allowing me the opportunity to explore my interest in corals from an early age, for tolerating my numerous 'experiments', the flipped circuit breakers, corroded tools, and occasional floods. Ruby Brackett, Tom Cole, Paul Fischer, Joann Montgomery, and Rusty Taylor, all of whom helped to enrich my education by supporting extracurricular academic activities. Leroy and Sally Jo Headley for maintaining the Geothermal Aquaculture Research Foundation where I had the opportunity to volunteer, work with a wide range of corals in captivity, and overcome any fears I may have had of applying superglue to living corals. The University of Idaho and especially Rolf Ingermann and Joseph Cloud who gave me an incredible opportunity to conduct independent research as an undergraduate, who were extremely supportive, and from whom I learned a great deal - I don't think I could have found a better introduction to research anywhere. The National Museum of Natural History, and my project advisors: John Pandolfi, Ann Budd, and Ian Macintyre who gave me my first introduction to coral paleo-proxies - particularly banding structures, trace element and isotopic compositions of the skeleton, which have been an interest ever since. The works of Peta Clode, William Fitt, Paola Furla, Joanie Kleypas, Alan Marshall, Francesca Marubini, Anders Meibom, and Lynne Whitehead which fueled my curiosity about coral calcification throughout my undergraduate education and continue to influence my research. The direction my research has taken over the last few years has in large part been shaped by my advisor, Anne Cohen, who has sent me in directions I would have never imagined my research taking when I first came in to the Joint Program. Little did I realize coming in that I would be spending winters diving in Woods Hole while ice floated on the surface of the water just to measure the winter time growth of a local coral, nor had I imagined that I would spend my time looking at crystal morphologies, and using crystal morphology to estimate saturation state within the calcifying environment of the coral and in turn trying to model saturation state variations within the calcifying environment. My advisor, Dan McCorkle also aided my work in ways I would have never imagined - who would have thought I'ld get a 3 month vacation from taking care of some 40 tanks of Astrangia poculata to go spend a winter on a tropical island off the coast of Australia, and doing so would actually be the suggestion of my advisor? If it weren't for Dan, it wouldn't have been possible to do studies in both locations at the same time. Dan also helped me to better understand and characterize carbonate chemistry, which has been critical for all of my projects. Both of my advisors have been very supportive of my work and have helped to improve it, especially the presentation of results. Thanks to the members of my thesis committee, Tanja Bosak, Glenn Gaetani, Ann Tarrant, and Scott Gallager have helped me over the past few years - Glenn helped greatly with inorganic aragonite studies, both with suggestions and use of his lab for the inorganic precipitation experiments and specimen preparation on which so much of my thesis is based, Ann has provided many valuable suggestions for my Astrangia work, and Scott has helped save me countless hours of data processing, made equipment available 
to me, not to mention helping to keep ESL running. Everyone involved in keeping ESL running, especially Molly Jacobs, Fred Keller, Kevin Thompson, and Amber York, as well as the security guards (Rick, Bill, Joe, Linda, Rob, John, Gary, Bill, Scot, Norman) who handle many a late night call to Fred or Kevin to take care of repairs, as well providing rides at odd hours. The WHOI dive program and divers - Stace Beaulieu, Lars Behrendt, Michael Brosnahan, Rod Catanach, Diane DiMassa, Scott Gallager, Greg Gerbi, Laura Hmelo, Erich Horgan, Annette M. Hynes, Pat Lohmann, Larry Madin, Scott McCue, Matthew McIlvin, Kelton McMahon, Anna Michel, Christine Mingione, Emily Miller, Todd Morrison, Byron Pedler, Kelly Rakow, Casey Saenger, Peter Schultz, Ann Tarrant, Nan Trowbridge, Kristen Whalen, Sandy Williams, Amber York, and Terry Rioux who have all helped with my various Astrangia projects. The academic programs office for helping to guide me through the logistics of the Joint Program and for financial support. Anne Hoggett, Lyle Vail and everyone at the Lizard Island Research Station who helped to provide one of the most rewarding experiences of my graduate career and in particular Lance and Maryann Pearce, Adel Heenan, Harriet Salomonsen, Dan Bayley, and Monica Gagliano. To Rebecca Gast, Dawn Moran, Mark Dennett and David Beaudoin for helping me with my initial work isolating sequences for aquaporins and calcium binding proteins and for microscopy assistance. To Jeffrey Hutter and Shailesh Nene for helping me to get started with AFM. To Rinat Gabitov for my first introduction to carrying out inorganic precipitation experiments and for samples. And to many others who have allowed me access to equipment, provided training and advice, analyzed samples, given me computer code, checked on my corals, edited rough drafts or helped improve presentations, which include: Fern Gibbons, Janelle Homburg, Greg Hirth, Nobumichi Shimizu, Stan Hart, Scot Birdwhistell, Sheri White, Benjamin Van Mooy, Simon Thorrold, Tom Kleindist, Jason Smith, Jerzy Blusztajn, David Wellwood, Rebecca Belastock, Fred Sayles, Joanne Goudreau, Paul Henderson, Justin Ries, Sharon Lamont, Sonya Dyhrman, Karen Casciotti, Neal Cantin, Ed Boyle, Andrew McDonnell, Jonathan Blythe, Erin Banning, Joanna Gyory, Elizabeth Orchard, Kelton McMahon, Casey Saenger, Meredith White, Evelyn Mervine, Emily Roland, Sharon Hoffman, Louis Kerr, Christina McGraw, Tim McClure, David Housman, Katie Boissonneault, Philip Alatalo, Mark St. Pierre, Darlene Ketten, Summer Praetorius, William Martin, Scott Doney, David Glover, David Kulis, Joan Bernhard, Jerry McManus, Alyson Bodendorf, Hana Keys, Ellen Roosen, Rick Galat, Alfonso Mucci, Holly Wichman, LuAnn Scott, and Susan Humphris. The administrative staff who have helped with so many of the day-day tasks: Christina Cuellar, Maryanne Ferreira, Lynn Stellrecht, Kelly Servant, Suellen Garner. To Katrina Goudkamp, the Great Barrier Reef Marine Park Authority, and other employees of the Australian government for permitting assistance and being responsive to questions. To WHOI computer and information systems personnel who have helped out with so many computer glitches over the years. My office mates who were always there, whether it be 6am or 1am, who created many an original song and who proved to me that even I can keep a plant alive, my fellow Joint Program students, my neighbors Ruth and Ryan and the 2004 MIT Biology cohort who provided endless diversions. To Carol Brackett for being there, or not there, and for, well, putting up with me. To all my friends and colleagues who have all helped to enrich my time here. And most 
importantly to Astrangia poculata a wonderful, amazing, and utterly confusing coral which has kept me busy for many years and will no doubt occupy my thoughts for years to come.

Funding for this research was provided by a National Science Foundation Graduate Research Fellowship, Academic programs, a MIT Presidential Fellowship, WHOI Interdisciplinary Award \#39040300, a WHOI Coastal Ocean Institute Award, the WHOI Ocean Life Institute, National Science Foundation grant \#OCE-0648157, a Lizard Island Doctoral Fellowship, and an International Society for Reef Studies/Ocean Conservancy Doctoral Fellowship. 


\section{Coral calcification: insights from inorganic experiments and coral responses to environmental variables}

\section{Introduction}

Despite decades of research, the role of symbiosis in coral calcification remains elusive.

It is well established that calcification rates increase in zooxanthellate corals during periods in which photosynthesis is occurring (Barnes and Chalker 1990). Comparisons

of facultatively symbiotic corals with and without zooxanthellae similarly show increased calcification in the presence of symbionts (Jacques et al., 1983). Yet, comparisons of zooxanthellate corals to azooxanthellate corals show that some azooxanthellate corals can grow just as rapidly as zooxanthellate corals, bleached corals are known to continue calcifying for some time at rates similar to those prior to bleaching, and in corals such as acroporids, the fastest growing regions are largely azooxanthellate (Gladfelter 1982; Marshall 1996; Mortensen and Rapp 1998; Rodrigues and Grottoli 2006). Thus, it is not clear that symbionts per se enhance calcification. There are several mechanisms which could be responsible for the increase in calcification often associated with zooxanthellae, ranging from changes in the synthesis of organic matrix components to changes in carbonate chemistry (e.g., Gattuso et al., 1999); here the focus will be on carbonate chemistry and saturation state. Calcification could increase due to photosynthate supplied by the zooxanthellae being used to fuel the transport of $\mathrm{Ca}^{2+}$ to the skeleton or protons away from the site of calcification (Goreau 1961), or due to the drawdown in $\mathrm{CO}_{2}$ as a direct result of photosynthesis, leading to increased $\mathrm{pH}$, shifting the dominate $\mathrm{CO}_{2}$ species from bicarbonate to carbonate ion and increasing the saturation state 
(Kawaguti and Sakumoto 1948). But, zooxanthellae may also limit calcification by competing for $\mathrm{CO}_{2}$, thus leading to a situation in which increased $\mathrm{pH}$ does not correspond to increased carbonate ion (Marubini and Davies 1996; Langdon and Atkinson 2005). To better understand different aspects of how zooxanthellae influence calcification, four projects were conducted to examine various aspects of the calcification process.

In Chapter 1 abiogenic aragonite precipitates formed under monitored conditions are compared with coral skeletons. The morphology and composition of the abiogenic precipitates are used to infer potential conditions under which the coral skeleton forms (Holcomb et al., 2009). Abiogenic aragonite precipitates from seawater as spherulites, with clusters of submicron granular materials occupying their centers and elongate (fibrous) needles radiating out to the edge. In each spherulite, the granular material formed first, at the highest fluid saturation state, whereas subsequent needle growth occurred at lower saturation state. The morphology of individual fibers also changes with saturation state, with fibers becoming thinner with increasing saturation state. Coral skeletons are shown to share the same crystal morphologies seen in abiogenic precipitates, with fine granular materials occupying centers of calcification and 'dark' bands which transect fibers, and fibrous aragonite radiating out from the centers of calcification. In addition to sharing morphological similarities, the elemental composition $(\mathrm{Mg} / \mathrm{Ca}$ and $\mathrm{Ba} / \mathrm{Ca}$ ratios) is shown to have similar patterns in both abiogenic and coral aragonite, with granular regions being enriched in trace elements relative to fibers. 
Based on the similar morphologies and compositions of centers of calcification and dark bands in corals, and centers and bands in abiogenic aragonite grains, I propose that granular crystal formation in corals is a result of high saturation state and that 'daily' banding patterns in corals reflect a cyclic change in saturation state.

In Chapter 2 skeletal growth on diurnal cycles is examined to (1) establish the timing of 'daily' band formation, and the relationship between band formation and photosynthesis in zooxanthellate corals and (2) to test the hypothesis that cyclic changes in saturation state could cause the observed banding patterns in corals. Calcium binding dyes were introduced to the seawater surrounding a coral to mark the skeleton formed while the dye was present. Patterns of dye incorporation suggest that crystal nucleation and growth have a diurnal cycle, with nucleation (dark band formation) occurring between dusk and dawn. In conjunction with skeletal extension data (Barnes and Crossland 1980; Vago et al., 1997), staining between dusk and dawn indicates that dark bands form near dusk, coinciding with maximal skeletal extension rates. Together with the abiogenic precipitation experiments (Chapter 1), this implies a high saturation state near dusk, to drive crystal nucleation. These observations can be explained by a conceptual model of coral calcification in which $\mathrm{CO}_{2}$ is limiting at the site of calcification during the day, and a non-carbonate buffer system in the calcifying environment allows a $\mathrm{CO}_{2}$ influx, associated with a decline in photosynthesis near dusk, to generate an increase in saturation state and thus drive crystal nucleation. 
In Chapter 3 the effects of $\mathrm{CO}_{2}$ and nutrients on the facultatively symbiotic coral Astrangia poculata are investigated. In zooxanthellate colonies, calcification is adversely affected by elevated $\mathrm{CO}_{2}$, but the addition of inorganic nutrients can reduce the impact of $\mathrm{CO}_{2}$ on calcification. This suggests that under elevated $\mathrm{CO}_{2}$ conditions, zooxanthellae can become nutrient limited, and thus less efficient at drawing down internal $\left[\mathrm{CO}_{2}\right]_{\mathrm{aq}}$. In this situation, adding inorganic nutrients could enable zooxanthellae to utilize more $\mathrm{CO}_{2}$, this enhanced photosynthesis may reduce $\left[\mathrm{CO}_{2}\right]_{\mathrm{aq}}$, and supply energy or alkalinity to help drive calcification.

In Chapter 4, the effects of $\mathrm{CO}_{2}$, nutrients, and temperature on the facultatively symbiotic coral Astrangia poculata are examined. No positive effect of nutrient addition was found for corals under elevated $\mathrm{pCO}_{2}$, potentially reflecting a lack of nutrient limitation of the zooxanthellae due to high ambient nutrient levels. In Chapter 3, ambient nutrient levels were $3 \pm 5 \mu \mathrm{M} \mathrm{NH}_{4}, 0.8 \pm 0.7 \mu \mathrm{M} \mathrm{NO}_{3}, 0.5 \pm 0.3 \mu \mathrm{M} \mathrm{PO}_{4}$, while for the treatment phase of Chapter 4, ambient nutrient levels were $1 \pm 0.4 \mu \mathrm{M} \mathrm{NH}_{4}, 4 \pm 0.4 \mu \mathrm{M} \mathrm{NO}_{3}, 0.6 \pm 0.1 \mu \mathrm{M} \mathrm{PO}_{4}$ for the $24^{\circ} \mathrm{C}$ tank, thus nitrate is consistently higher during the treatment phase of Chapter 4. Phosphate though slightly higher, also shows greater variability in Chapter 3 , reflecting intervals with low and high phosphate concentrations, so the apparent positive effect of nutrients under $\mathrm{CO}_{2}$ enriched conditions found in Chapter 3 may be driven by intervals in which ambient nutrient levels are low enough to be limiting. In Chapter 4, coral calcification generally decreased in response to $\mathrm{CO}_{2}$, but the pattern differed greatly between male and female corals, with reproductively active female corals being much 
more sensitive to $\mathrm{CO}_{2}$ than males. This suggests a greater cost for egg production relative to sperm production which takes resources away from calcification, potentially limiting energy availability for calcification. If this pattern applies to gonochoric coral species under natural conditions, rising atmospheric $\mathrm{CO}_{2}$ levels could lead to a greater reduction in the growth rates of female corals, making them more vulnerable to disturbance events. With successive disturbance events and reduced recovery rates of female corals, gender ratios could shift toward male dominated reefs, potentially reducing rates of sexual reproduction, making corals more vulnerable to ocean acidification than initial changes in calcification rates would suggest. 


\section{References:}

Barnes DJ, Chalker BE (1990) Calcification and photosynthesis in reef building corals and algae. in coral reefs ed Z Dubinsky. 109-131

Barnes DJ, Crossland CJ (1980) Diurnal and seasonal variations in the growth of a staghorn coral measured by time-lapse photography. Limnol. Oceanography 25: 1113-1117

Cohen AL, McConnaughey TA (2003) Geochemical perspectives on coral mineralization. Reviews in Mineralogy Geochem: Biomineralization 54: 151-187

Gattuso JP, Allemand D, Frankignoulle M (1999) Photosynthesis and calcification at cellular, organismal and community levels in coral reefs: a review of interactions and control by carbonate chemistry. American Zoologist 39: 160-183

Gladfelter EH (1983) Skeletal development in Acropora cervicornis II. diel patterns of calcium carbonate accretion. Coral Reefs 2: 91-100

Goreau TF (1961) On the relation of calcification to primary productivity in reef building organisms. In The biology of hydra and some other coelenterates: Lenhoff, H.M., Loomis, W.H. Ed pp. 269-285

Jacques TG, Marshall N, Pilson MEQ (1983) Experimental ecology of the temperate scleractinian coral Astrangia danae II. Effect of temperature, light intensity and symbiosis with zooxanthellae on metabolic rate and calcification. Marine Biol. 76: $135-148$

Kawaguti S, Sakumoto D (1948) The effect of light on the calcium deposition of corals. Bull. Oceanograpaical Inst. Taiwan: 65-70

Kleypas JA, Buddemeier RW, Archer D, Gattuso JP, Langdon C, Opdyke BN (1999) Geochemical consequences of increased atmospheric carbon dioxide on coral reefs. Science 284: 118-120

Langdon C, Atkinson MJ (2005) Effect of elevated pCO2 on photosynthesis and calcification of corals and interactions with seasonal change in temperature/irradiance and nutrient enrichment. J. Geophys. Res. Ocean

Marshall AT (1996) Calcification rates in corals. Science 274: 117-118

Marubini F, Davies PS (1996) Nitrate increases zooxanthellae population density and reduces skeletogenesis in corals. Marine Biol. 127: 319-328

Mortensen PB, Rapp HT (1998) Oxygen and carbon isotope ratios related to growth line patterns in skeletons of Lophelia pertusa (L) (Anthozoa, scleractinia): implications for determination of linear extension rates. Sarsia 83: 433-446

Muscatine L (1990) The role of symbiotic algae in carbon and energy flux in reef corals. in coral reefs ed Z Dubinsky. 75-87

Risk MJ, Pearce TH (1992) Interference imaging of daily growth bands in massive corals. Nature 358: 572-573

Rodrigues LJ, Grottoli AG (2006) Calcification rate and the stable carbon, oxygen, and nitrogen isotopes in the skeleton, host tissue, and zooxanthellae of bleached and recovering Hawaiian corals. Geochim. Cosmochim. Acta 70: 2781-2789

Vago R, Gill E, Collingwood JC (1997) Laser measurements of coral growth. Nature 386: $30-31$ 
Chapter 1: Compositional and morphological features of aragonite precipitated experimentally from seawater and biogenically by corals

\begin{abstract}
The morphology and composition of abiogenic (synthetic) aragonites precipitated experimentally from seawater and the aragonite accreted by scleractinian corals were characterized at the micron scale. The synthetic aragonites precipitated from supersaturated seawater solutions as spherulites, typically 20-100 $\mu \mathrm{m}$ in diameter, with clusters of submicron granular materials occupying their centers and elongate (fibrous) needles radiating out to the edge. Using Sr isotope spikes, the formation of the central granular material was shown to be associated with high fluid $\mathrm{pH}$ and saturation state whereas needle growth occurred at lower $\mathrm{pH}$ and saturation state. The granular regions have significantly higher $\mathrm{Mg} / \mathrm{Ca}$ and $\mathrm{Ba} / \mathrm{Ca}$ ratios than the surrounding fibers. $\mathbf{S r}$

Two types of crystals are identified in the coral skeleton: sub-micron granular material and elongate (fibrous) crystals that radiate out from the granular regions. Granular materials are found in "centers of calcification" and in fine bands that transect the fibers. They have significantly higher $\mathrm{Mg} / \mathrm{Ca}$ and $\mathrm{Ba} / \mathrm{Ca}$ ratios than the surrounding fibers.

The observed relationship between seawater saturation state and crystal morphology and composition in the synthetic aragonites was used as a framework to interpret observations of the coral skeleton. We propose that coral skeletal growth can be viewed as a cyclical process driven by changes in the saturation state of the coral's calcifying fluids. When saturation state is high, granular crystals precipitate at the tips of the existing skeletal elements forming the centers of calcification. As the saturation state decreases, aragonitic fibers grow, radiating out from the centers of calcification.
\end{abstract}

Published as: M. Holcomb, A.L. Cohen, R.I. Gabitov and J.L. Hutter, Compositional and morphological features of aragonite precipitated experimentally from seawater and biogenically by corals. Geochimica et Cosmochimica Acta. v. 73, p. 4166-4179, 2009.

doi:10.1016/j.gca.2009.04.015 


\section{INTRODUCTION}

Early studies of the ultrastructure of the scleractinian coral skeleton identified two distinct structures: the centers of calcification which appear as dark spots in petrographic thin-section, and the fibrous crystals which radiate out from the centers (e.g., Ogilvie, 1896; Vaughan and Wells, 1943; Wells, 1956). The centers of calcification and their bundles of fibers are called sclerodermites and are considered to be the basic building blocks of the skeleton (Wells, 1956). Bryan and Hill (1941) noted the striking similarity between the spherulitic morphology observed in a range of mineral systems, and coral sclerodermites. Both exhibit fibrous crystals radiating from a common center.

Spherulitic growth in mineral systems is associated with diffusion-controlled growth from highly supersaturated solutions (Keith and Padden, 1963; Chernov, 1984). Such observations led Barnes (1970) and Constantz (1986), among others, to describe the precipitation of aragonite by scleractinian corals as a process analogous to crystal growth from highly supersaturated solutions.

Over the past few decades, a range of imaging and analytical techniques have been employed to examine coral skeletons. SEM imaging of materials occupying the centers of calcification showed that these are morphologically distinct from the surrounding fibers. The materials at the centers of calcification have been variously described as small, nano-crystals, very fine, or granular (e.g., Wainwright, 1964; Constantz, 1986; Cohen et al., 2001; Clode and Marshall, 2003). Selective analyses of centers of calcification and adjacent fibers indicate that centers of calcification are also 
compositionally distinct. Several elements, notably $\mathrm{Mg}, \mathrm{Sr}, \mathrm{S}, \mathrm{Ba}$, and $\mathrm{N}$, are enriched in the centers of calcification (e.g., Cuif et al., 2003; Gagnon et al., 2007; Meibom et al., 2004, 2006, 2007) and several isotope ratios, such as $\delta^{13} \mathrm{C}, \delta^{18} \mathrm{O}$, and $\delta^{11} \mathrm{~B}$, are depleted in centers of calcification (e.g., Adkins et al., 2003; Rollion-Bard et al., 2003; Meibom et al., 2006; Blamart et al., 2007) relative to the surrounding fibers. These findings have been used to support the hypothesis that centers of calcification are formed by a process distinct from that responsible for the formation of fibers (e.g., Meibom et al., 2006) and, thus, the formation of aragonite by corals is a process distinct from the inorganic precipitation of aragonite from a highly supersaturated solution. Lacking from these studies, however, is a comparison between coral aragonite and synthetic aragonite precipitated from a highly supersaturated solution.

Here, results from such a study are presented. A range of imaging techniques: light microscopy, fluorescence microscopy, scanning electron microscopy (SEM) and atomic force microscopy (AFM), as well as elemental measurements using secondary ion mass spectrometry (SIMS) were employed to characterize the morphology and chemistry of synthetic (i.e. precipitated experimentally from seawater) and biogenic aragonites (i.e. precipitated by tropical corals).

\section{METHODS}

\subsection{Synthetic Aragonite Precipitates}

Techniques employed for precipitating synthetic aragonites from seawater were adopted from Kinsman and Holland (1969), as modified by Gaetani and Cohen (2006). 
The details for some of the experiments employed in this study differ slightly, so the specifics of each experiment are briefly described below.

Aragonite was precipitated from $0.45 \mu \mathrm{m}$ filtered Vineyard Sound (Woods Hole, MA, USA) seawater (salinity 30.8-32.1). A PTFE or PET beaker containing $600 \mathrm{ml}$ of filtered seawater was placed into a Lauda RE-106 isothermal bath, and stirred continuously with a PTFE stirrer. Note that initial experiments showed no effect of the plastic type on the development of a run, so later runs used transparent PET which allowed the onset of crystallization to be more readily assessed. Several different experiments were conducted, each representing one of three categories of experiments, described below.

\subsubsection{Timing of Precipitate Formation}

To establish the relationship between fluid chemistry, crystal morphology and crystal composition, experiment 1 used a pair of Sr isotope spikes to constrain the timing of precipitation. In experiment 1 , seawater was held at $25^{\circ} \mathrm{C}$ in a PTFE beaker and stirred continuously at $40 \mathrm{rpm}$. Evaporated seawater and a $0.04 \mathrm{M} \mathrm{Na}_{2} \mathrm{CO}_{3}$ (Alfa Aesar) solution were introduced simultaneously at a rate of $4.8 \mathrm{ml} \mathrm{h}^{-1}$ for 37.5 hours. Evaporated seawater was prepared by placing seawater in a PTFE beaker held in a $80^{\circ} \mathrm{C}$ water bath (for some experiments, concentrated seawater was prepared in a polypropylene beaker held at $60^{\circ} \mathrm{C}$ - similar results were obtained with both methods) until half the mass had been lost. The evaporated seawater was used to maintain the salinity at a roughly constant value as the $0.04 \mathrm{M} \mathrm{Na}_{2} \mathrm{CO}_{3}$ solution was added (initial 
salinity 31.85 , final salinity 31.88 ). During this experiment, strontium isotope spikes $\left({ }^{84} \mathrm{Sr}\right.$ and $\left.{ }^{86} \mathrm{Sr}\right)$ were introduced at separate times into the seawater mixture. These spikes are incorporated into the growing crystals and serve as a marker from which the timing and rate of crystal growth can be determined. After the first $65 \mathrm{ml}$ were added, $100 \mu \mathrm{l}$ of ${ }^{84} \mathrm{Sr}$ solution was added to nearly double the ${ }^{84} \mathrm{Sr}$ concentration. After the final $\mathrm{Na}_{2} \mathrm{CO}_{3}$ addition, $100 \mu \mathrm{l}$ of ${ }^{86} \mathrm{Sr}$ was added to approximately double the ${ }^{86} \mathrm{Sr}$ concentration. Precipitation of aragonite was allowed to proceed for an additional 24 hours after pumping stopped. Precipitates were separated from solution by filtration through a 0.7 $\mu \mathrm{m}$ glass fiber filter and rinsed briefly with distilled water and ethanol. Sr isotopes were purchased from Oak Ridge National Lab.

\subsubsection{Cyclic Saturation States}

In experiments 2 and 3, the saturation state was cycled over the course of each run by pulsed addition of $\mathrm{Na}_{2} \mathrm{CO}_{3}$ in an attempt to produce precipitates with alternating regions of growth formed at high and low saturation states (bands). Experiment 2 was conducted under variable salinity conditions at either $55^{\circ} \mathrm{C}$ or $65^{\circ} \mathrm{C}$. A $0.01 \mathrm{M} \mathrm{Na}_{2} \mathrm{CO}_{3}$ solution was added to the beaker of seawater in two $125 \mathrm{ml}$ steps, 1 day apart, at a rate of $\sim 1 \mathrm{ml} \mathrm{min}{ }^{-1}$, and the solution was stirred at $120 \mathrm{rpm}$. This experiment is described in detail by Gaetani and Cohen (2006). In Experiment 3, a $\sim 0.04 \mathrm{M} \mathrm{Na}_{2} \mathrm{CO}_{3}$ solution and evaporated seawater were added in two steps to seawater (initial salinity 31.3 , final 32.1 ) held in a PET beaker in a $25^{\circ} \mathrm{C}$ water bath. The solution was stirred continuously at 120 rpm. ${ }^{84} \mathrm{Sr},{ }^{86} \mathrm{Sr}$ and ${ }^{137} \mathrm{Ba}$ isotope spikes were added at different times over the course of 
each run. Pumping durations, volumes, times of isotope spike addition and solution chemistry are given in the electronic annex Table EA1, Fig. EA1 and EA2.

\subsubsection{Effect of Pumping Rate on Morphology}

To examine the effects of saturation state on the morphology of precipitates, experiments 4, 5, 6 and 7 were conducted using different pumping rates to generate different 'steady state' conditions under which the bulk of the precipitate formed in each experiment. In experiments 4, 5 and 6, a syringe pump was used to continuously add a $\sim 0.04 \mathrm{M} \mathrm{Na}_{2} \mathrm{CO}_{3}$ solution and evaporated seawater to seawater in a PTFE beaker (final salinities ranged from 31.4-32.8). Experiments 4-6 were conducted at $25^{\circ} \mathrm{C}$ while stirring at 120rpm. Pumping rates for the bulk of each experiment were: high $=360 \mathrm{ml} \mathrm{h}^{-}$

${ }^{1}$ for experiment 4 , intermediate $=2 \mathrm{ml} \mathrm{h}^{-1}$ for experiment 5 , and low $=0.2 \mathrm{ml} \mathrm{h}^{-1}$ for experiment 6. Injection volumes ranged from 76 to $181 \mathrm{ml}$. Aragonite seed crystals (fish otoliths, cleaned by sonication and ground to 5-300 $\mu \mathrm{m}$ ) were added to experiment 6 in an effort to speed nucleation. Precipitation was allowed to continue for 21-35 h after pumping stopped.

In experiment 7, a 0.04 $\mathrm{M} \mathrm{Na}_{2} \mathrm{CO}_{3}$ solution and evaporated seawater were added continuously at a rate of $0.4 \mathrm{ml} / \mathrm{hr}$ to seawater (initial salinity 31.3 , final 33.5 ) held in a PET beaker in a $25^{\circ} \mathrm{C}$ water bath. The solution was stirred continuously at $120 \mathrm{rpm}$. Isotope spikes $\left({ }^{84} \mathrm{Sr},{ }^{86} \mathrm{Sr}\right.$, and $\left.{ }^{137} \mathrm{Ba}\right)$ were added at different times over the course of the run. Pumping durations, volumes, times of isotope spike addition and solution chemistry are given in the Table EA1, Fig. EA1 and EA2. 


\subsubsection{Growth Conditions of the Synthetic Aragonites}

Solution pH (NBS scale) was monitored throughout aragonite synthesis experiments 1, 3, 4, 5, 6, and 7 (Table 1, Fig. 1a, electronic annex Table EA-1, Fig. EA1). Saturation state was also determined periodically by measuring alkalinity and calcium concentration, data are presented in Fig. 1a, Table EA-1 and Figure EA-1. Solution $\mathrm{pH}$ increased as $\mathrm{Na}_{2} \mathrm{CO}_{3}$ was added until nucleation occurred. Following nucleation, solution $\mathrm{pH}$ fell to a quasi-steady state value related to pumping rate (Fig. EA-1, EA-2). At the end of each experiment, when pumping of the $\mathrm{Na}_{2} \mathrm{CO}_{3}$ solution ceased, the $\mathrm{pH}$ of each experimental solution dropped but remained above the initial value ( 8.0). Saturation states follow a trend similar to that seen in $\mathrm{pH}$ data (Table. EA1). Solution chemistry for some of the runs and starting materials are provided in Table EA-1. Raman spectra of the precipitates reveal a peak at $\sim 705 \mathrm{~cm}^{-1}$, consistent with the presence of aragonite (e.g., Clarkson et al., 1992). Raman data are presented in Fig. EA3.

\subsection{Coral Samples}

The skeletons of three coral species Diploria labyrinthiformis (brain coral), Porites lutea and Porites solida were examined. D. labyrinthiformis (sample \#BER002) was collected live from John Smith's Bay, Bermuda at a depth of $13 \mathrm{~m}$ (Cohen et al., 2004). Porites lutea (sample \#JA4) was collected live from Johnston Atoll, north central Pacific (Cohen et al., 2001). Porites solida (sample \#141-B05-53) was provided by Dr 
David Barnes (AIMS). Porites solida was collected live in June 1989 from the Great Barrier Reef, Australia $\left(151^{\circ} \mathrm{E}, 21^{\circ} \mathrm{S}\right)($ Cohen and Hart, 1997).

\subsection{Polishing of Synthetic and Coral Aragonites}

All samples were placed inside $2.5 \mathrm{~cm} \mathrm{Al} \mathrm{rings} \mathrm{and} \mathrm{imbedded} \mathrm{in} \mathrm{Epo-thin} \mathrm{epoxy}$ (Buehler) prior to polishing. Double sided adhesive tape (Buehler) was used to hold specimens and rings in place as epoxy was added. Corals were prepared by breaking $\sim 1$

$\mathrm{cm}^{2}$ sections of coral skeleton, roughly parallel to the axis of growth, and placing the sections on the adhesive. Synthetic aragonites were mounted by applying a light dusting of grains to the adhesive. Polishing was done on nylon cloths with alumina (Mark V Laboratories) suspended in heptane (Alfa Aesar), except for the coral used for light microscopy and SIMS analysis, which was prepared commercially (OMNI Laboratories). A small amount of water was used to initially apply the alumina grit. Each specimen was polished through a range of grit sizes down to $0.3 \mu \mathrm{m}$, or $0.05 \mu \mathrm{m}$ for specimens imaged with Atomic Force Microscopy. Following mechanical polishing, a final polish with 0.02 $\mu \mathrm{m}$ colloidal silica was applied to all samples, with the exception of those used in the acridine staining experiments (see below). Colloidal silica is a water-based polishing compound, hence there is dissolution of the aragonite in addition to mechanical abrasion. An initial comparison of different techniques revealed that a colloidal silica etch on the synthetic grains gave good AFM images (Fig. EA-4). In addition to the dissolution induced by the colloidal silica, corals were etched for 30-50 s prior to AFM imaging using $0.1 \%$ formic acid and 3\% gluteraldehyde in water (Cuif et al., 2003). Etching was 
stopped by rinsing with water. This additional etching step improved contrast between features in coral samples, as observed in preliminary comparisons of different techniques (Fig. EA-5). Samples for SEM imaging were either mounted on SEM stubs without any further preparation, or were polished as above and etched for $10 \mathrm{~s}$ with $0.1 \mathrm{~N} \mathrm{HCl}$.

\subsection{Imaging:}

\subsubsection{Light Microscopy and Scanning Electron Microscopy (SEM)}

Polished sections of corals and synthetic aragonites were examined in reflected and transmitted light with a Nikon Eclipse E 600 Polarizing microscope equipped with a Spot Insight color CCD camera. SEM imaging of Au/Pd coated samples (both polished and unpolished) was done using a JEOL 840 scanning electron microscope. Accelerating voltage was $15 \mathrm{kV}$ unless otherwise specified.

\subsubsection{Acridine Orange Staining}

Acridine orange staining of polished sections of corals and synthetic aragonites was carried out following the protocol of Stolarski (2003). Acridine orange is a dye used to stain coral skeletons, and regions retaining acridine orange are thought to have high concentrations of organic materials. Corals were etched prior to staining by placing samples in distilled water overnight to lightly etch the surface. Samples were overlain with a $1 \%$ acridine orange (Alfa Aesar) aqueous solution, allowed to sit for 5 minutes, briefly rinsed, blotted dry and imaged on a Zeiss Axiovert inverted microscope using a mercury vapor UV source and FITC short pass filter set. Images were captured using either a Sony color CCD camera or a Canon Digital Rebel XTi camera. 


\subsubsection{Atomic Force Microscopy (AFM)}

AFM imaging (which measures small changes in the height of a surface) of polished sections of corals and synthetic aragonites was conducted at The University of Western Ontario using a Veeco MultiMode AFM and at Woods Hole Oceanographic Institution using a Veeco Dimension 3100 AFM equipped with silicon nitride tips (Veeco NP-S, with a $\sim 10 \mathrm{~nm}$ radius of curvature, and BudgetSensors, with a $\sim 20 \mathrm{~nm}$ radius of curvature, respectively). All images were acquired in contact mode. Image capture and processing were performed using Digital Instruments NanoScope software.

\subsection{Secondary Ion Mass Spectrometry (SIMS ion microprobe)}

Metal $(\mathrm{M}) / \mathrm{Ca}(\mathrm{Mg} / \mathrm{Ca}, \mathrm{Sr} / \mathrm{Ca}$, and $\mathrm{Ba} / \mathrm{Ca})$ ratios of the coral Porites lutea and synthetic aragonite formed in experiment 1 were analyzed with a Cameca 3f Ion Microprobe. Following a 3-minute pre-burn to remove the gold coating, a single spot on the coral sample was illuminated with the primary ion beam while measuring secondary ion intensities for ${ }^{24} \mathrm{Mg},{ }^{88} \mathrm{Sr},{ }^{138} \mathrm{Ba}$ and ${ }^{42} \mathrm{Ca}$ (Gaetani and Cohen, 2006). A $2.5 \mathrm{nA} \mathrm{O}$ primary ion beam, $\sim 10 \mu \mathrm{m}$ in diameter, was accelerated at $12.7 \mathrm{keV}$. Secondary ion intensities were measured using a $-80 \mathrm{eV}$ offset from the peak of the energy distribution. This energy filtering reduces molecular interferences to $<0.1 \%$ (Hart and Cohen, 1996).

In addition to the $\mathrm{M} / \mathrm{Ca}$ ratios, ${ }^{84} \mathrm{Sr} /{ }^{88} \mathrm{Sr}$ and ${ }^{86} \mathrm{Sr} /{ }^{88} \mathrm{Sr}$ ratios were also determined in the synthetic aragonite grains in order to locate the isotope spikes. Individual synthetic aragonite grains were targeted with a $4 \mathrm{nA} \mathrm{O-primary}$ ion beam, $\sim 7 \mu \mathrm{m}$ in diameter, accelerated at $12.7 \mathrm{keV}$. Secondary ion intensities $\left({ }^{24} \mathrm{Mg},{ }^{84} \mathrm{Sr},{ }^{86} \mathrm{Sr},{ }^{88} \mathrm{Sr},{ }^{138} \mathrm{Ba}\right.$ and $\left.{ }^{40} \mathrm{Ca}\right)$ were measured using a $-90 \mathrm{eV}$ offset from the peak of the energy distribution. Ion probe 
intensity ratios were converted to molar ratios using the carbonatite standard OKA assuming an intercept of zero and a linear response. OKA was assumed to be homogeneous with a $\mathrm{Mg} / \mathrm{Ca}$ ratio of $4.47 \mathrm{mmol} / \mathrm{mol}$, a Sr/Ca ratio of $19.3 \mathrm{mmol} / \mathrm{mol}$ and a $\mathrm{Ba} / \mathrm{Ca}$ ratio of $1.61 \mathrm{mmol} / \mathrm{mol}$ (Gaetani and Cohen, 2006), isotopic abundances were assumed to be similar for both OKA and aragonite. At least eight measurements of the OKA standard were made each day samples were measured, average intensity ratios measured at the time of coral data collection were: $0.202,2.62$ and 0.16 for ${ }^{24} \mathrm{Mg} /{ }^{42} \mathrm{Ca}$, ${ }^{88} \mathrm{Sr} /{ }^{42} \mathrm{Ca}$ and ${ }^{138} \mathrm{Ba} /{ }^{42} \mathrm{Ca}$ respectively; at the time of synthetic aragonite measurements, values were: $0.0018,0.014$ and 0.00072 for ${ }^{24} \mathrm{Mg} /{ }^{40} \mathrm{Ca},{ }^{88} \mathrm{Sr} /{ }^{40} \mathrm{Ca}$ and ${ }^{138} \mathrm{Ba} /{ }^{40} \mathrm{Ca}$ respectively. In all cases, standard errors were less 3\%. Means were compared using a ttest (Zar, 1984).

\section{RESULTS}

\subsection{Synthetic Spherulites}

The individual grains formed in experiment 1 are roughly circular; in polarized light, the center $(c)$ of each grain is distinguished by a dark region (Fig 1c). Radiating out from this center are aragonite fibers $(f)$. The morphology of the grains is consistent with the spherulitic morphology found in a range of minerals i.e. a radially disposed array of acicular crystals that emerge from a common center or nucleation region (e.g., Cross, 1891; Iddings 1891), typical of crystals formed rapidly from a supersaturated or supercooled solution (Lofgren, 1971; Sunagawa, 1987; Lowenstam and Weiner, 1989). Using SEM (Fig 1e), it can be seen that the material found in the center is sub-micron in 
size and has a granular $(g)$ appearance, while the fibers $(f)$ radiating out from the center are $\sim 1 \mu \mathrm{m}$ wide and several microns long.

\subsubsection{Timing of Growth}

The $\mathrm{Sr}$ isotope ratios in the center of each grain formed during experiment 1 reflect natural abundances (Fig. 1d), indicating that the centers formed prior to the addition of the ${ }^{84} \mathrm{Sr}$ isotope spike. The ${ }^{84} \mathrm{Sr}$ spike is present in the fibrous aragonite between the centers and the edge of the spherulite, indicating that fibers formed after the centers but prior to the addition of ${ }^{86} \mathrm{Sr}$ (Fig. 1d). High ${ }^{86} \mathrm{Sr}$ was found only at the edges of a few spherulites, indicating that very little aragonite precipitated following the addition of ${ }^{86} \mathrm{Sr}$ (data not shown).

The presence of spikes allows the crystal morphology to be correlated with the solution chemistry. In experiment 1 , solution $\mathrm{pH}$ increased as $\mathrm{Na}_{2} \mathrm{CO}_{3}$ was added until nucleation occurred (Fig. 1a). The granular centers of the aragonite grains formed during this high $\mathrm{pH}$ period ( $\mathrm{pH} \sim 9.2$ ), as indicated by the absence of the ${ }^{84} \mathrm{Sr}$ spike. Following nucleation, solution $\mathrm{pH}$ fell to a quasi-steady state value related to pumping rate. Fibrous aragonite grew during this period when $\mathrm{pH}$ was $\sim 8.9$, as indicated by the presence of the

${ }^{84} \mathrm{Sr}$ but absence of ${ }^{86} \mathrm{Sr}$ spikes (Fig. 1b, c and d). Very little aragonite was deposited near the end of the experiment after pumping stopped when $\mathrm{pH}$ was $\sim 8.6$, as indicated by the scarcity of elevated ${ }^{86} \mathrm{Sr}$.

\subsubsection{Stepped Pumping Produces Banded Spherulites}


Fig. 2a represents a cross section through a composite of three synthetic aragonite grains formed in experiment 2 at $65^{\circ} \mathrm{C}$. Fig. $2 \mathrm{~b}$ shows a cross section through a single synthetic aragonite grain formed in experiment 3 at $25^{\circ} \mathrm{C}$. The two pumping cycles used in each of these experiments lead to two cycles of high and low saturation state over the course of aragonite precipitation. The synthetic aragonite grains precipitated in these experiments are similar to those in Fig. 1c, but an additional feature, a single dark band running perpendicular to the axis of fiber growth, is present (Fig. 2a,b). In these spherulites, the dark band is located $\sim 15 \mu \mathrm{m}$ from the outer edge of each grain formed in experiment 2 , and $\sim 4 \mu \mathrm{m}$ from the edge for grains formed in experiment 3 .

Fig. 2c shows a fluorescence image of a synthetic aragonite grain, grown in experiment 2 by cyclic pumping, stained with acridine orange. Addition of acridine orange results in increased fluorescence associated with the center $(c)$ and dark bands (arrow) observed in light microscopy.

Fig. 2d shows an SEM image of a polished, $\mathrm{HCl}$ etched aragonite grain formed in experiment 3 by cyclic pumping. The central region is composed of granular to finely fibrous material. Fine fibers radiate out from the center and become larger till reaching the band (arrow). Following the band, fibers again radiate out to the edge of the aragonite grain.

\subsubsection{SEM Imaging}

SEM images of synthetic aragonites precipitated in experiment 4 (Fig. 3a), 5 (Fig 3b), 6 (seeded, Fig. 3d) and 7 (Fig. 3c) at high (Fig. 3a), medium (Fig. 3b), and low (Fig. 
$3 \mathrm{c}, \mathrm{d})$ pumping rates reveal a systematic change in crystal morphology with pumping rate, and thus $\mathrm{pH}$ (Fig. EA 1,2). Synthetic aragonite grains formed in the high pumping rate run ( pH 9.5) lack well-defined crystals, and are composed of very fine fibers (Fig. 3a). Synthetic grains grown at intermediate pumping rates $(\mathrm{pH} \sim 8.8)$ are composed of welldefined aragonite blades, 1-2 $\mu \mathrm{m}$ wide and several microns long and there are clear grain boundaries between individual fibers (Fig. 3b). Synthetic aragonites precipitated in the low pumping rate experiments ( $\mathrm{pH} \sim 8.2$ for Fig. $3 \mathrm{c}, \sim 8.3$ for Fig. $3 \mathrm{~d}$ ) are composed of broad ( 2 $\mu \mathrm{m}$ wide), highly faceted fibers that are widely separated (Fig. 3c,d). The fibers emerge from a common center, as shown in Fig. 3c and the inset in Fig. 3d.

\subsubsection{AFM Imaging}

AFM height images of synthetic aragonite grains etched with colloidal silica show similar changes in crystal morphology with pumping rate to that seen with SEM (Fig. 4). In all images, height is on a scale of $0-400 \mathrm{~nm}$, with the highest regions shown as white, lowest as black. The length-scale, in microns, is shown on the $\mathrm{x}$ and $\mathrm{y}$ axes of each image.

In Fig. 4a, an AFM image of two synthetic aragonite grains precipitated with two stepped additions of sodium carbonate (experiment 2 ) is shown. The grains have roughly circular centers $(c)$ (only partly visible at the base of the image) surrounded by two layers of fibrous aragonite $(f)$. A darker region of granular material, $\sim 10 \mu \mathrm{m}$ wide (arrow), separates the inner and outer fibrous layers. Granular materials are sub-micron in size, while fibers are micron scale features - typically $8 \mu \mathrm{m}$ long, and $0.7 \mu \mathrm{m}$ wide. 
Fig. $4 \mathrm{~b}$ shows a synthetic aragonite formed in a high pumping rate run $(\mathrm{pH} \sim 9.5$, Exp. 4). The entire surface of the grain is rough and granular $(g)$, lacking defined fibers. In Fig. 4c, a synthetic aragonite formed in a low pumping rate ( $\mathrm{pH} \sim 8.3$, Exp. 6 ) run is imaged. Granular material $(g)$ is restricted to near the center of the grain. The bulk of the grain is made up of broad well-defined fibers $(f)$ that emerge from the granular materials and radiate outward to the edge (Fig $4 \mathrm{c}$ ).

\subsection{5. $M /$ Ca Ratios}

$\mathrm{Mg} / \mathrm{Ca}, \mathrm{Sr} / \mathrm{Ca}$, and $\mathrm{Ba} / \mathrm{Ca}$ ratios in centers and fibers of synthetic aragonites grown in experiment 1 were measured by SIMS ion microprobe (Fig. 5). Discrete analyses of centers of synthetic grains were possible with the $7 \mu \mathrm{m}$ diameter analytical spot. Similarly, discrete analyses of aragonite fibers emerging from the centers of synthetic aragonite grains were made. The $\mathrm{Mg} / \mathrm{Ca}$ and $\mathrm{Ba} / \mathrm{Ca}$ ratios are higher in the centers of the grains $(6.35 \pm 0.09$ and $0.022 \pm 0.001 \mathrm{mmol} / \mathrm{mol}$ respectively) than in the surrounding fibers $(5.01 \pm 0.07$ and $0.013 \pm 0.001 \mathrm{mmol} / \mathrm{mol}$ respectively $)(\mathrm{p}<0.01)$. $\mathrm{Sr} / \mathrm{Ca}$ ratios show no significant difference between centers $(10.2 \pm 0.1 \mathrm{mmol} / \mathrm{mol})$ and fibers $(10.1 \pm 0.1 \mathrm{mmol} / \mathrm{mol})$.

\subsection{Coral aragonite}

\subsubsection{Light Microscopy}

In Fig. 6a, the arrangement of aragonite crystals in a coral skeleton is seen in a cross section through a synapticulum or horizontal cross-bar of Porites solida. In 
polarized light, the center of calcification $(c)$ appears dark with poorly defined edges. Radiating outward from the center of calcification are aragonite fibers $(f)$. This observation is consistent with the description of coral sclerodermites (Wells, 1956). In this section, the fibers are interrupted by fine dark bands (arrow), $\sim 2 \mu \mathrm{m}$ apart and aligned perpendicular to the axis of crystal extension.

\subsubsection{Fluorescence Imaging}

Fig. 6b shows a fluorescence image of a section of the coral Diploria labyrinthiformis stained with acridine orange. There is an increase in fluorescence associated with the centers of calcification $(c)$ and dark bands (arrow) following acridine staining. This observation is consistent with that of Stolarski (2003).

\subsubsection{SEM Imaging}

Fig. 7 shows SEM images of dissepiments (horizontal sheets) in the skeletons of Diploria labyrinthiformis (Fig. 7a) and Porites lutea (Fig. 7b). The dissepiments are composed of two layers (as identified by Barnes, 1971). The primary (base) layer is built of small granular materials $(g),<1 \mu \mathrm{m}$ diameter. The secondary layer is composed of broad blade-like fibers $(f)$, each $\sim 1-2 \mu \mathrm{m}$ wide and several microns long.

\subsubsection{AFM Imaging}

In Figure 8, AFM height images of skeletal cross sections of Diploria (Fig. 8a,c) and Porites (Fig. 8b,d) reveal centers of calcification as regions of low relief (dark) and bands of aragonite fibers as regions of higher relief (light). Fine dark bands that transect 
the aragonite fibers are also visible as regions of low relief. Materials occupying centers of calcification (Fig. 8d) and dark bands (Fig. 8c) are small and granular in texture. Conversely, in both species, the light bands are packed with larger elongated fibers

In Figure 8a, a cross section through a Diploria septotheca, the direction of vertical growth is from the lower right to the upper left of the image. Centers of calcification $(c)$ appear crescent-shaped, and form a line of discrete dark crescents up the middle of the septum. Centers of calcification are continuous with fine dark bands (arrow) that extend toward the edge of the septotheca. The width of the fine bands decreases with distance from the centers of calcification. Near the centers of calcification, the fine bands are $5.3 \pm 0.4 \mu \mathrm{m}(\mathrm{n}=10)$ wide. At the outer edge of the septotheca, the width of the bands is not discernible. As the width of the dark bands decrease, the distinction between light bands of aragonite fibers and the dark bands that interrupt them becomes less distinct. Near the center of the septotheca, the transition between light fibrous bands and fine dark bands is abrupt. Toward the edge of the septotheca, the fibers appear continuous, cutting across dark bands.

In the Porites septum, the centers of calcification are oval rather than crescent shaped (Fig. 8b). Fine dark bands (arrows) are present in the fibers but these are not as clearly defined in this Porites specimen as they are in the Diploria skeleton.

\subsection{5. $M / C a$ Ratios}

Selective analysis of $\mathrm{Mg} / \mathrm{Ca}, \mathrm{Sr} / \mathrm{Ca}$, and $\mathrm{Ba} / \mathrm{Ca}$ ratios in centers of calcification and surrounding fibers of a Porites skeleton are shown in Figure 9. $\mathrm{Mg} / \mathrm{Ca}, \mathrm{Sr} / \mathrm{Ca}$, and $\mathrm{Ba} / \mathrm{Ca}$ ratios are higher in the centers of calcification $(4.91 \pm 0.02,8.98 \pm 0.03$, and 0.0058 
$\pm 0.0001 \mathrm{mmol} / \mathrm{mol}$ respectively) than in adjacent fibers $(3.88 \pm 0.01,8.63 \pm 0.03$, and $0.0034 \pm 0.0001 \mathrm{mmol} / \mathrm{mol}$ respectively). For all $\mathrm{M} / \mathrm{Ca}$ ratios measured, the value for the centers was significantly higher than for the fibers $(\mathrm{p}<0.01)$.

\section{DISCUSSION}

\subsection{Synthetic Precipitates}

Synthetic aragonite crystal morphology and composition are coupled to the $\mathrm{pH}$ and saturation state of the fluid from which the crystals grew. Within a single spherulite, the centers packed with sub-micron sized granular materials form when the saturation state of the fluid is very high. Growth of fibrous crystals outward from the centers occurs when the saturation state of the fluid has decreased following nucleation. The supersaturation achieved prior to nucleation depends on the rate at which $\mathrm{Na}_{2} \mathrm{CO}_{3}$ is added; the faster the addition of $\mathrm{Na}_{2} \mathrm{CO}_{3}$, the higher the saturation state achieved prior to nucleation, consistent with the work of Prieto and others $(1989,1994)$. In addition, the morphology of the spherulite (i.e., open, coarse versus closed, fine) and the size and shape of the aragonite needles within the spherulites change systematically with the $\mathrm{pH}$ (saturation state) of the seawater in which they grew. In the high $\mathrm{pH}$, high saturation state experiments, fine closed spherulites form that contain densely packed fibers with illdefined grain boundaries (Fig 3a). Conversely, spherulites formed at low $\mathrm{pH}$ are typically open and coarse, containing fewer, broad, faceted fibers (Fig 3b). This observation is

consistent with systematic variations in crystal morphologies observed in non- $\mathrm{CaCO}_{3}$ minerals and polymers with increasing degrees of supercooling or with increasing supersaturation (e.g., Keith and Padden, 1963; Lofgren, 1971, 1974; Chernov, 1984; 
Sunagawa, 1987), and reflects the crystal morphology that allows the maximum growth rate under those conditions (e.g., Tiller, 1964). The formation of smooth sides and development of facets in the lower pumping rate experiments likely reflects a change in growth mechanism, with a rough interface associated with spherulitic growth transitioning to a smooth interface dominated by dislocation growth controlling step flow on faceted crystals (e.g., Sunagawa, 1981, 1987; Prieto et al., 1989).

Measurements of $\mathrm{Mg} / \mathrm{Ca}, \mathrm{Sr} / \mathrm{Ca}$, and $\mathrm{Ba} / \mathrm{Ca}$ show that the $\mathrm{Mg} / \mathrm{Ca}$ and $\mathrm{Ba} / \mathrm{Ca}$ ratios are significantly elevated at the center of the spherulite relative to the fibers (Fig 5). In each experiment, the highest saturation state occurs at the onset of nucleation, thus the center of each spherulite contains crystals expected to have the highest growth/precipitation rate (Burton and Walter, 1987) (though it should be noted that growth mechanism and relative areas of different crystal faces also change, and could influence composition as well). This relationship between crystal growth rate and M/Ca ratio is consistent with the growth rate dependency expected for $\mathrm{Mg} / \mathrm{Ca}$ and $\mathrm{Ba} / \mathrm{Ca}$, as all $\mathrm{M} / \mathrm{Ca}$ ratios are expected to increase with higher growth rates due to more efficient entrapment of an impurity enriched mineral surface layer composition at higher crystal growth rates (Watson 2004; Gaetani and Cohen, 2006). Gabitov et al. (2006; 2008) showed that the growth dependence of $\mathrm{Mg} / \mathrm{Ca}$ in aragonite is much higher than that of $\mathrm{Sr} / \mathrm{Ca}$. Therefore, the absence of a significant elevation of $\mathrm{Sr} / \mathrm{Ca}$ in the centers found in this study is consistent with their data.

\subsection{Coral Aragonite}


Data presented here concur with earlier observations that coral sclerodermites consist of aragonite needles radiating out from regions of fine granular materials or 'nanocrystals' (Fig 6, 7, 8) (Vaughan and Wells, 1943; Wainwright, 1964; Constantz, 1986; Cohen et al., 2001; Clode and Marshall, 2003). The sub-micron-sized granular materials are found at the base of dissepiments (Fig. 7a,b), in the centers of calcification (Fig. 8) and in the fine bands that cut across aragonite fibers (Fig. 8).

Regions associated with granular materials are also associated with an increase in fluorescence following acridine orange staining (Fig. 6b), which is consistent with the results of Gautret et al. (2000) and Stolarski (2003). $\mathrm{Mg} / \mathrm{Ca}, \mathrm{Sr} / \mathrm{Ca}$, and $\mathrm{Ba} / \mathrm{Ca}$ ratios are found to increase at the centers of calcification relative to adjacent fibers (Fig. 9), which is consistent with the findings of Meibom et al. $(2004,2006)$.

\subsection{Results of Inorganic Precipitation Experiments in Relation to Coral Skeleton Morphology and Composition}

Aragonite crystals formed by experimental precipitation from a supersaturated seawater solution and the aragonite crystals formed by living corals during skeletogenesis share several morphological and compositional features. Both the synthetic aragonites precipitated in this study and the coral sclerodermites are composed of two distinct types of crystals: submicron-sized granular materials and larger elongate fibrous crystals that radiate out from the granular materials. Granular materials are found near the centers of synthetic grains, in the centers of calcification of corals, and in dark bands found both in corals and in the synthetic aragonites formed by stepped additions of $\mathrm{Na}_{2} \mathrm{CO}_{3}$. Higher 
$\mathrm{Mg} / \mathrm{Ca}$ and $\mathrm{Ba} / \mathrm{Ca}$ ratios and acridine orange staining correspond to regions of granular materials in both synthetic aragonite (Fig. 2c, 5) and corals (Fig. 6b, 9), consistent with the results of Gautret et al. (2000) and Meibom et al. (2006) for corals.

Since the composition and morphology of crystals provide insights into the conditions under which they grew (e.g., Lofgren, 1971; Reddy and Nancollas, 1976; Marsh, 1988; Prieto et al., 1997; Marsh, 1998; Cohen and McConnaughey, 2003; Tong et al., 2004; Wasylenski et al., 2005), the observed crystal morphologies and compositions associated with known conditions in the synthetic experiments may be useful in assigning possible conditions of formation of naturally formed precipitates.

The similarity between the finely fibrous to granular materials at the centers of spherulites (Fig. 4a), grains formed at very high pH (Fig 4b) and materials occupying centers of calcification, fine bands, and the base layer of dissepiments in coral skeletons (Fig. 8), suggests that these regions in the coral represent material formed at substantially elevated saturation states. The morphology of fibrous aragonite in coral skeletons (Fig. $7 \mathrm{a}, \mathrm{b}$ ) is consistent with that of aragonites grown in the mid pumping (moderate precipitation) rate experiment (Fig. 3b): tightly spaced but with distinct boundaries between fibers. This may suggest that fibrous growth in corals occurs at a saturation state below that needed to induce nucleation but substantially above that of ambient seawater.

\subsubsection{Band Formation in Corals and Synthetic Aragonite}

The formation of alternating bands of fibrous crystals and granular materials in synthetic aragonites precipitated in experiments 2 and 3 is consistent with the stepped 
addition of $\mathrm{Na}_{2} \mathrm{CO}_{3}$ which caused the saturation state of the seawater solution to cycle during the experiment. Addition of the first volume of $\mathrm{Na}_{2} \mathrm{CO}_{3}$ elevated the solution $\mathrm{pH}$ (supersaturation), initiating nucleation and the formation of granular materials at the center of the spherulites. Following nucleation, the solution $\mathrm{pH}$ dropped, enabling fibers to grow and radiate outward from the center. Addition of a second volume of $\mathrm{Na}_{2} \mathrm{CO}_{3}$ solution elevated the solution $\mathrm{pH}$, favoring nucleation over elongation of pre-existing crystals, forming a fine band of granular materials followed again by radial growth (Fig 4a).

Such a cycling of fluid saturation state may explain the formation of microscale bands of alternating fibers and granular materials within the coral skeleton (Jell, 1974; Sorauf and Jell, 1977; Risk and Pearce, 1992). The crystal morphologies reported here (Fig 8a,b,c) are consistent with repeated cycles of high and moderate saturation states. Zooxanthellate coral calcification rates (e.g., Kawaguti and Sakumoto, 1948; Goreau, 1959; Barnes and Chalker, 1990), and the internal pH of the coral (Al-Horani et al., 2003), are both known to increase substantially during the day and fall at night. Daytime $\mathrm{pH}$ values within the coral have been measured in excess of 9.0 (Al-Horani et al., 2003). Thus, the microscale bands (often called daily growth bands) within zooxanthellate coral skeletons may be the product of substantially elevated daytime saturation states and more moderate night time saturation states.

A model to explain the banding patterns seen in Diploria is presented in Fig. 10. In Diploria, the banding patterns are characterized by thick regions of granular material near the center of a skeletal element, with a gradual thinning of the granular band away 
from the center, transitioning to fibrous growth and a loss of bands (Fig. 8a). This pattern could be explained by the presence of a highly supersaturated fluid adjacent to where the granular centers form, transitioning to a moderate saturation state far from the centers, where fibers are continuous. The region of highest saturation state would be associated with the fastest growth and, thus, the thickest band of granular material. Moving down that saturation state gradient, growth would slow, shifting from granular to fibrous material. At different times (perhaps at night), a more uniform, moderate, saturation state could exist throughout the calcifying environment, generating a band of fibrous crystals. Variations in rates of ion pumping or internal fluid flow could generate both spatial and temporal variations in saturation state.

The proposed cycle in saturation state could account for both the high $\mathrm{Mg} / \mathrm{Ca}$ ratios at centers of calcification, and the alternating micron-scale bands of high and low $\mathrm{Mg} / \mathrm{Ca}$ ratios reported by Meibom et al. (2004). This is consistent with the suggestion of Tsukamoto and Tsukamoto (1996) that growth rate variation could account for some of the variability in $\mathrm{Mg} / \mathrm{Ca}$ ratios and is similar to the explanation proposed by Eggins et al. (2004), which attributes daily $\mathrm{Mg} / \mathrm{Ca}$ bands in foraminifera to cycles in $\mathrm{pH}$.

Cycles in the saturation state of the calcifying environment can also give rise to fine-scale heterogeneity in isotope ratios. Differences in $\delta^{13} \mathrm{C}$ and $\delta^{18} \mathrm{O}$ between centers and fibers, as well as fine scale heterogeneity in the composition of coral fibers may, in part, be due to diffusion kinetics favoring the lighter isotope in the faster growing regions (Rollion-Bard et al., 2003; Meibom et al., 2006). The calcification model proposed by Adkins et al. (2003) expands upon the diffusion model to incorporate multiple carbon 
sources which contribute to skeletal $\delta^{13} \mathrm{C}$ in a $\mathrm{pH}$ dependent manner. Similarly elevations in the $\mathrm{pH}$ of the calcifying fluid induce depletion of $\delta^{18} \mathrm{O}$. This model is compatible with the compositional response of aragonite precipitates to variations in saturation state proposed here. In the Adkins et al. (2003) model, high $\mathrm{pH}$ in the calcifying environment is associated with light $\delta^{13} \mathrm{C}$ and $\delta^{18} \mathrm{O}$ and rapid growth rate. Assuming that saturation state of the precipitating fluid is linked to $\mathrm{pH}$, centers of calcification and bands of granular crystals should have a lighter $\delta^{13} \mathrm{C}$ and $\delta^{18} \mathrm{O}$ signature relative to fibers.

Some independent observations appear to contradict the proposed model. For example, Blamart et al. (2007) show that centers of calcification have lower boron isotope ratios than adjacent fibers, which the authors interpret to mean that crystals in centers of calcification form at a lower $\mathrm{pH}$ than the surrounding fibers. However, factors controlling the boron isotope ratios of coral skeletons are not well-understood and considerable variability has been found between different coral species, even when grown under similar environmental conditions (e.g., Vengosh et al., 1991; Pagani et al., 2005; Klochko et al., 2006, 2009; Xiao et al., 2006; Blamart et al., 2007). In two separate studies, Clode and Marshall (2002) and Tambutte et al. (2007) examined the interface between the skeleton and the calicoblastic ectoderm, and found, in freeze-fractured specimens, that the membrane lays flush with the skeletal surface with the possible exception of nanometer scale spaces. Tambutte et al. (2007) interpreted this as the absence of a reservoir in which saturation state could be regulated but that nanometer scale volumes are sufficient for saturation state to control crystal formation cannot be ruled out. 
The results presented here show that features of coral skeletons such as: banding patterns, fibrous and granular materials, $\mathrm{Mg} / \mathrm{Ca}$ ratios, and acridine fluorescence patterns, can be reproduced by manipulating the saturation state. Whereas cycles in saturation state may not be the only factor controlling the morphology and chemistry of coral crystals, our results suggest that these features cannot be used as firm evidence of organic materials (e.g., templates) controlling crystal growth. By using synthetic aragonite spherulites as a control material, it may be possible to identify coral features that cannot be reproduced by manipulating saturation state alone and, thus, may be indicative of biological control over and above controlling saturation state within the calcifying environment.

\subsection{Differences}

Despite the numerous similarities between synthetic aragonites and coral skeletons, there are some key differences. The most significant of these for sample preparation is the greater apparent solubility of the synthetic aragonites (Fig EA4, 5). This may be due to coral-derived organic materials decreasing the solubility of the coral skeleton or it may be a reflection of the much smaller size of individual spherulites $(<100 \mu \mathrm{m}$ diameter for a spherulite, versus millimeters for a coral), making them more vulnerable to dissolution.

\section{SUMMARY}


The morphology and composition of synthetic aragonitic spherulites precipitated experimentally from seawater is shown to change systematically with the $\mathrm{pH}$ (saturation state) of the precipitation environment. Spherulites progress from coarse open structures at $\mathrm{pH}$ values near seawater to fine closed structures at high $\mathrm{pH}$. The highest $\mathrm{pH}$ precipitates have a granular appearance, similar to the granular appearance of centers of calcification and bands in corals, whereas coral fibers resemble synthetic fibers grown at lower $\mathrm{pH}$. Variations in elemental ratios coincide with the morphological variations, suggesting that the saturation state influences both the morphology and composition of the precipitate. The similarities, observed between portions of the coral skeleton and synthetic aragonites grown under varying controlled, chemical conditions, may prove useful in interpreting the coral biomineralization process. These observations suggest that the saturation state beneath the calicoblastic epithelium is not homogenous, and that there may be regular cycles in the saturation state within the calcifying environment which lead to the formation of centers of calcification and fine bands within the coral skeleton.

Acknowledgments. The authors are grateful for the insightful comments of AE Alfonso Mucci and the anonymous reviewers that greatly improved the manuscript, to Shailesh Nene (UWO) for assistance with AFM imaging, Louie Kerr (MBL) for help on the SEM, and Nobuchimi Shimizu (WHOI) for help with SIMS analysis Jerzy Blustajn, Zhengrong Wang and Scot Birdwhistell for assistance with ICP-MS, David Wellwood for salinities, and Joanne Goudreau for alkalinity data. Greg Hirth and Glenn Gaetani (WHOI) 
provided insightful discussions and materials. Glenn Gaetani made his laboratory available for sample preparation. Sheri White, Dawn Moran, Becky Gast, Karen Casciotti, and Ben Van Mooy (WHOI) provided equipment that made this work possible. Financial support was provided by a WHOI Interdisciplinary Award \#39040300, a WHOI Coastal Ocean Institute Award to MH, and by NSF OCE-0648157. This material is based upon work supported under a National Science Foundation Graduate Research Fellowship. 


\section{REFERENCES}

Adkins J.F., Boyle E.A., Curry W.B. and Lutringer A. (2003) Stable isotopes in deep-sea corals and a new mechanism for vital effects. Geochim. Cosmochim. Acta 67, 1129-1143.

Al-Horani F.A., Al-Moghrabi S.M. and de Beer D. (2003) The mechanism of calcification and its relation to photosynthesis and respiration in the scleractinian coral Galaxea fascicularis. Mar. Biol. 142: 419-426.

Barnes D.J. (1970) Coral skeletons: an explanation of their growth and structure. Science 170: $1305-1308$.

Barnes D.J. (1971) A study of growth, structure and form in modern coral skeletons. Ph.D. thesis, Univ. Newcastle upon Tyne.

Barnes D.J. and Chalker B.E. (1990) Calcification and photosynthesis in reef building corals and algae. In Coral Reefs: Ecosystems of the World, Vol. 25 (ed Z. Dubinsky, D.W. Goodall) Elsevier. pp 109-131.

Blamart D., Rollion-Bard C., Meibom A., Cuif J.-P., Juillet-Leclerc A. and Dauphin Y. (2007) Correlation of boron isotopic composition with ultrastructure in the deepsea coral Lophelia pertusa: Implications for biomineralization and paleo-pH, Geochem. Geophys. Geosyst., 8: Q12001.

Bryan W.H. and Hill D. (1941) Spherulitic crystallization as a mechanism of skeletal growth in the hexacorals. Proc. R. Soc. Queensland LII: 78-92.

Burton E.A. and Walter L.M. (1987) Relative precipitation rates of aragonite and Mg calcite from seawater: Temperature or carbonate ion control? Geology 15: 111114.

Chernov A.A. (1984) Modern Crystallography III Crystal Growth. Springer-Verlag, New York.

Clarkson J.R., Price T.J. and Adams C.J. (1992) Role of metastable phases in the spontaneous precipitation of calcium carbonate. J. Chem. Soc. Faraday Trans. 88: 243-249.

Clode P.L., and Marshall A.T. (2002). Low temperature FESEM of the calcifying interface of a scleractinian coral. Tissue and Cell 34: 187-198.

Clode P.L. and Marshall A.T. (2003) Skeletal microstructure of Galaxea fascicularis exsert septa: a high-resolution SEM study. Biol. Bull. 204: 146-154.

Cohen A.L. and Hart S.R. (1997) The effect of colony topography on climate signals in coral skeleton. Geochim. Cosmochim. Acta 61: 3905-3912.

Cohen A.L., Layne G.D., Hart S.R. and Lobel P.S. (2001) Kinetic control of skeletal $\mathrm{Sr} / \mathrm{Ca}$ in a symbiotic coral: Implications for the paleotemperature proxy. Paleoceanography 16: 20-26.

Cohen A.L. and McConnaughey T.A. (2003) Geochemical perspectives on coral mineralization. Rev. Mineral. Geochem. Biomineralization 54: 151-187. 
Cohen A.L., Smith S.R., McCartney M.S. and van Etten J. (2004) How brain coral record climate: an integration of skeletal structure, growth and chemistry of Diporia labrynthiformis from Bermuda. Mar. Ecol. Prog. Ser. 271: 147-158.

Constantz B.R. (1986) Coral skeleton construction: a physiochemically dominated process. Palaios 1: 152-157.

Cross W. (1891) Constitution and origin of spherulites in acid eruptive rocks. Washington Philosophical Society Bulletin 11: 411-444.

Cuif J.-P., Dauphin Y., Doucet J., Salome M. and Susini J. (2003) XANES mapping of organic sulfate in three scleractinian coral skeletons. Geochim. Cosmochim. Acta 67: $75-83$.

Eggins S.M., Sadekov A. and Deckker P.D. (2004) Modulation and daily banding of $\mathrm{Mg} / \mathrm{Ca}$ in Orbulina universa tests by symbiont photosynthesis and respiration: a complication for seawater thermometry? Earth Planet. Sci. Lett. 225: 411-419.

Gabitov R.I., Cohen A.L., Gaetani G.A., Holcomb M. and Watson E.B. (2006) The impact of crystal growth rate on element ratios in aragonite: an experimental approach to understanding vital effects. Geochim. Cosmochim. Acta 70s:A187.

Gabitov R.I., Watson E.B., Cohen A.L., Gaetani G.A. and Ehrlich H.L. (2008)

Experimental determination of temperature and growth rate effects on U6+ and $\mathrm{Mg} 2+$ partitioning between aragonite and fluid. Geochim. Cosmochim. Acta 72: 4058-4068.

Gaetani G.A. and Cohen A.L. (2006) Element partitioning during precipitation of aragonite from seawater: a framework for understanding paleoproxies. Geochim. Cosmochim. Acta 70: 4617-4634.

Gagnon A.C., Adkins J.F., Fernandez D.P., and Robinson L.F. (2007) Sr/Ca and Mg/Ca vital effects correlated with skeletal architecture in a scleractinian deep-sea coral and the role of Rayleigh fractionation. Earth. Planet. Sci. Lett. 261: 280-295.

Gautret P., Cuif J.-P. and Stolarski J. (2000) Organic components of the skeleton of scleractinian corals - evidence from in situ acridine orange staining. Acta Palaeontologica Polonica 45: 107-118.

Goreau T.F. (1959) The physiology of skeleton formation in corals I. a method for measuring the rate of calcium deposition by corals under different conditions. Biol. Bull. 116: 59-75.

Hart S.R. and Cohen A.L. (1996) An ion probe study of annual cycles of Sr/Ca and other trace elements in corals. Geochim. Cosmochim. Acta 60: 3075-3084.

Iddings J.P. (1891) Spherulitic crystallization. Wash. Philosoph. Soc. Bull. 11:445-463.

Jell J.S. (1974) The microstructure of some scleractinian corals. Proc. 2nd Int. Coral Reef Sym. 2:301-320.

Kawaguti S., Sakumoto D., (1948) The effect of light on the calcium deposition of corals. Bull. Oceanograpaical Inst. Taiwan 4: 65-70.

Keith H.D. and Padden F.J.J. (1963) A Phenomenological theory of spherulitic crystallization. J. Appl. Phys. 34: 2409-2421.

Kinsman D.J.J. and Holland H.D. (1969) The co-precipitation of cations with CaCO3 IV. The co-precipitation of Sr2+ with aragonite between 16 and 96 C. Geochim. Cosmochim. Acta 33: 1-17. 
Klochko K., Kaufman A.J., Yao W., Byrne R.H. and Tossell J.A. (2006) Experimental measurement of boron isotope fractionation in seawater. Earth. Planet. Sci. Lett. 248: $276-285$.

Klochko K., Cody G.D., Tossell J.A., Dera P., Kaufman A.J. (2009) Re-evaluating boron speciation in biogenic calcite and aragonite using ${ }^{11}$ B MAS NMR. Geochim. Cosmochim. Acta 73: 1890-1900.

Lofgren G. (1971) Spherulitic textures in glassy and crystalline rocks. J. Geophys. Res. 76: $5635-5648$.

Lofgren G. (1974) An experimental study of plagioclase crystal morphology: isothermal crystallization. Am. J. Sci. 274: 243-273.

Lowenstam H.A. and Weiner S. (1989) On biomineralization. Oxford University Press, New York p 207-251.

Marsh B.D. (1988) Crystal size distribution (CSD) in rocks and the kinetics and dynamics of crystallization I theory. Contrib Mineral. Petrol. 99: 277-291.

Marsh B.D. (1998) On the Interpretation of Crystal Size Distributions in Magmatic Systems. J. Petrol. 39: 553-599.

Meibom A., Cuif J.-P., Hillion F., Constantz B.R., Juillet-Leclerc A., Dauphin Y., Watanabe T. and Dunbar R.B. (2004) Distribution of magnesium in coral skeleton. Geophys. Res. Lett. 31: L23306.

Meibom A., Yurimoto H., Cuif J.-P., Domart-Coulon I., Houlbreque F., Constantz B., Dauphin Y., Tambutte E., Tambutte S., Allemand D., Wooden J. and Dunbar R. (2006) Vital effects in coral skeletal composition display strict three dimensional control. Geophys. Res. Lett. 33:L1160.

Meibom A, Mostefaoui S, Cuif J-P, Dauphin Y, Houlbreque F, Dunbar R, and Constantz B (2007) Biological forcing controls the chemistry of reef-building coral skeleton. Geophys. Res. Lett. 34:L02601.

Ogilvie M.M. (1896) Microscopic and Systematic Study of Madreporarian Types of Corals. Phil. Trans. R. Soc. Lond. B 187: 83-345.

Pagani M., Lemarchand D., Spivack A. and Gaillardet, J., (2005) A critical evaluation of the boron isotope-pH proxy: The accuracy of ancient ocean $\mathrm{pH}$ estimates. Geochim. Cosmochim. Acta 69, 953-961.

Prieto M., Fernandez-Diaz L. and Lopez-Andres S. (1989) Supersaturation evolution and first precipitate location in crystal growth in gels; application to barium and strontium carbonates. J. Crystal Growth 98, 447-460.

Prieto M., Putnis A., Fernandez-Diaz L. and Lopez-Andres S. (1994) Metastability in diffusing-reacting systems. J. Crystal Growth 142, 225-235.

Prieto M., Fernandez-Gonzalez A., Putnis A. and Fernandez-Diaz L. (1997) Nucleation, growth, and zoning phenomena in crystallizing $(\mathrm{Ba}, \mathrm{Sr}) \mathrm{CO} 3, \mathrm{Ba}$ (SO4,CrO4), (Ba,Sr) $\mathrm{SO} 4$, and $(\mathrm{Cd}, \mathrm{Ca}) \mathrm{CO}$ solid solutions from aqueous solutions. Geochim. Cosmochim. Acta 61: 3383-3397.

Reddy M.M. and Nancollas G.H. (1976) The crystallization of calcium carbonate: IV. The effect of magnesium, strontium and sulfate ions. J. Cryst. Growth 35: 33-38.

Risk M.J., and Pearce T.H. (1992) Interference imaging of daily growth bands in massive corals. Nature 358: 572-573. 
Rollion-Bard C., Blamart D., Cuif J.-P. and Juillet-Leclerc A. (2003) Microanalysis of C and $\mathrm{O}$ isotopes of azooxanthellate and zooxanthellate corals by ion microprobe. Coral Reefs 22: 405-415.

Sorauf J.E. and Jell J.S. (1977) Structure and incremental growth in the ahermatypic coral Desmophyllum cristagalli from the North Alantic. Paleontology 20:1-19.

Stolarski J. (2003) Three-dimensional micro- and nanostructural characteristics of the scleractinian coral skeleton: A biocalcification proxy. Acta Palaeontologica Polonica 48: 497-530.

Sunagawa I. (1981) Characteristics of crystal growth in nature as seen from the morphology of mineral crystals. Bull. Mineral. 104: 81-87.

Sunagawa I. (1987) Morphology of Crystals Part B. Terra Scientific Publishing Co. Tokyo

Tambutté E., Allemand D., Zoccola D., Meibom A., Lotto S., Caminiti N. and Tambutté S. (2007) Observations of the tissue-skeleton interface in the scleractinian coral Stylophora pistillata. Coral Reefs 26:517-529.

Tiller W.A. (1964) Dendrites. Science 146: 871-879.

Tong H., Ma W., Wang L., Wan P., Hu J. and Cao L. (2004) Control over the crystal phase, shape, size and aggregation of calcium carbonate via a L-aspartic acid inducing process. Biomaterials 25: 3923-3929.

Tsukamoto S. and Tsukamoto H. (1996) Variability of trace elements in a daily banded coral. Geographical Report Tokyo Metropolitan U 31: 167-172.

Vaughan T.W., and Wells J.W. (1943) Revision of the suborders families, and genera of the scleracinia. Geological Society of America special papers 44.

Vengosh A., Kolodny Y., Starinsky A., Chivas A.R. and McCulloch M.T. (1991) Coprecipitation and isotopic fractionation of boron in modern biogenic carbonates. Geochim. Cosmochim. Acta 55: 2901-2910.

Wainwright S.A. (1964) Studies of the mineral phase of coral skeleton. Exp Cell Res 34: 213-230.

Wasylenki L.E., Dove P.M. and Yoreo J.J.D. (2005) Effects of temperature and transport conditions on calcite growth in the presence of Mg2: Implications for paleothermometry. Geochim. Cosmochim. Acta 69: 4227-4236.

Watson E.B. (2004) A conceptual model for near-surface kinetic controls on the traceelement and stable isotope composition of abiogenic calcite crystals. Geochim. Cosmochim. Acta 68: 1473-1488.

Wells J.W. (1956) Scleractinia. In Treatise on invertebrate paleontology: 328-444.

Xiao Y.K., Shirodkar P.V., Zhang C.G., Wei H.Z., Liu W.G. and Zhou W.J. (2006) Isotopic fractionation of boron in growing corals and its palaeoenvironmental implication. Current Science 90: 414-420.

Zar J.H. (1984) Biostatistical analysis. Prentice-Hall, Englewood Cliffs, NJ. 
Table 1. pH (NBS scale) values measured near the time of nucleation, and following nucleation while pumping continued (representing the period during which most growth occurred) for each synthetic precipitation run. Nucleation represents the peak in $\mathrm{pH}$ values, while growth is an average of $\mathrm{pH}$ values measured following nucleation, standard deviation is given in parenthesis.

Run

Exp. 1.

Exp. 4. high pumping rate

Exp. 5. intermediate pumping rate

Exp. 6. low pumping rate

Exp. 7. low pump rate

\begin{tabular}{cr} 
Nucleation & \multicolumn{1}{c}{ Growth } \\
9.2 & $8.9(.1)$ \\
9.6 & $9.5(.1)$ \\
9.2 & $8.8(.2)$ \\
8.9 & $8.3(.2)$ \\
8.7 & $8.16(.02)$
\end{tabular}



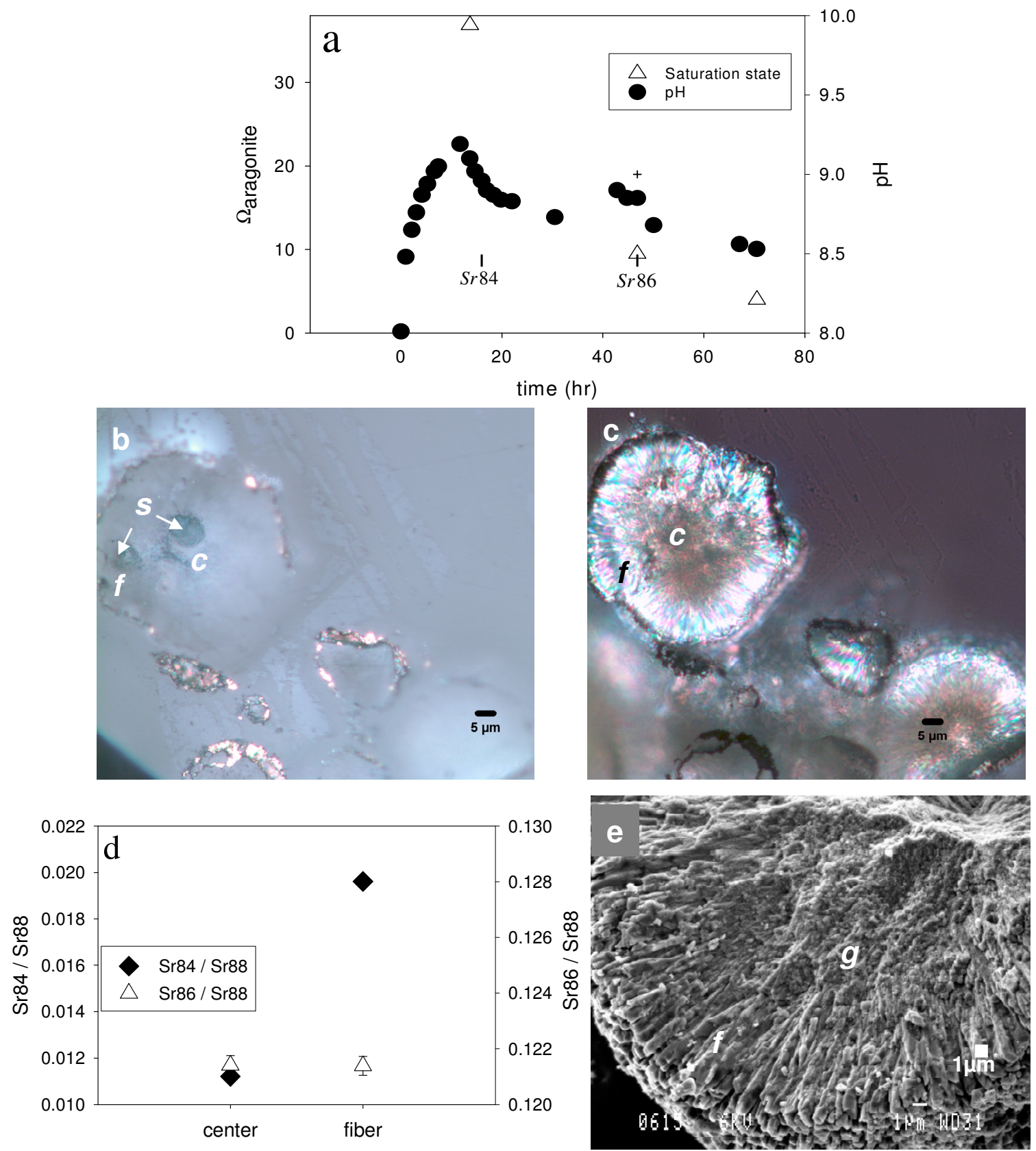

Figure 1. Precipitation conditions, light microscopy images, isotope ratios, and SEM images for grains formed in Experiment 1. a: Saturation state (open triangles) and $\mathrm{pH}$ (black circles) over the course of Experiment 1, the time at which pumping stopped is 
indicated by the cross, the times at which isotope spikes were added are indicated by vertical lines labeled with the isotope added. Errors are small relative to symbol size. b: A grain with ionprobe spots $(s)$ near the center $(c)$, and in a fibrous region $(f)$ imaged with reflected light. c: The same grain shown in b, imaged with cross-polarized transmitted light, which better shows the fibers $(f)$. d: Sr isotope ratios determined for the same spots used for M/Ca data in Figure 4. Sr84/88 ratios (black circles) are significantly higher in the fibers than the centers ( $\mathrm{p}<0.01$ ), while Sr86/88 ratios (open triangles) show no significant difference. Points are means, error bars are standard error. e: SEM image of a grain formed in Experiment 1, which shows fibers $(f)$ radiating out from a granular center $(g)$. Accelerating voltage for this image is $6 \mathrm{kV}$. 

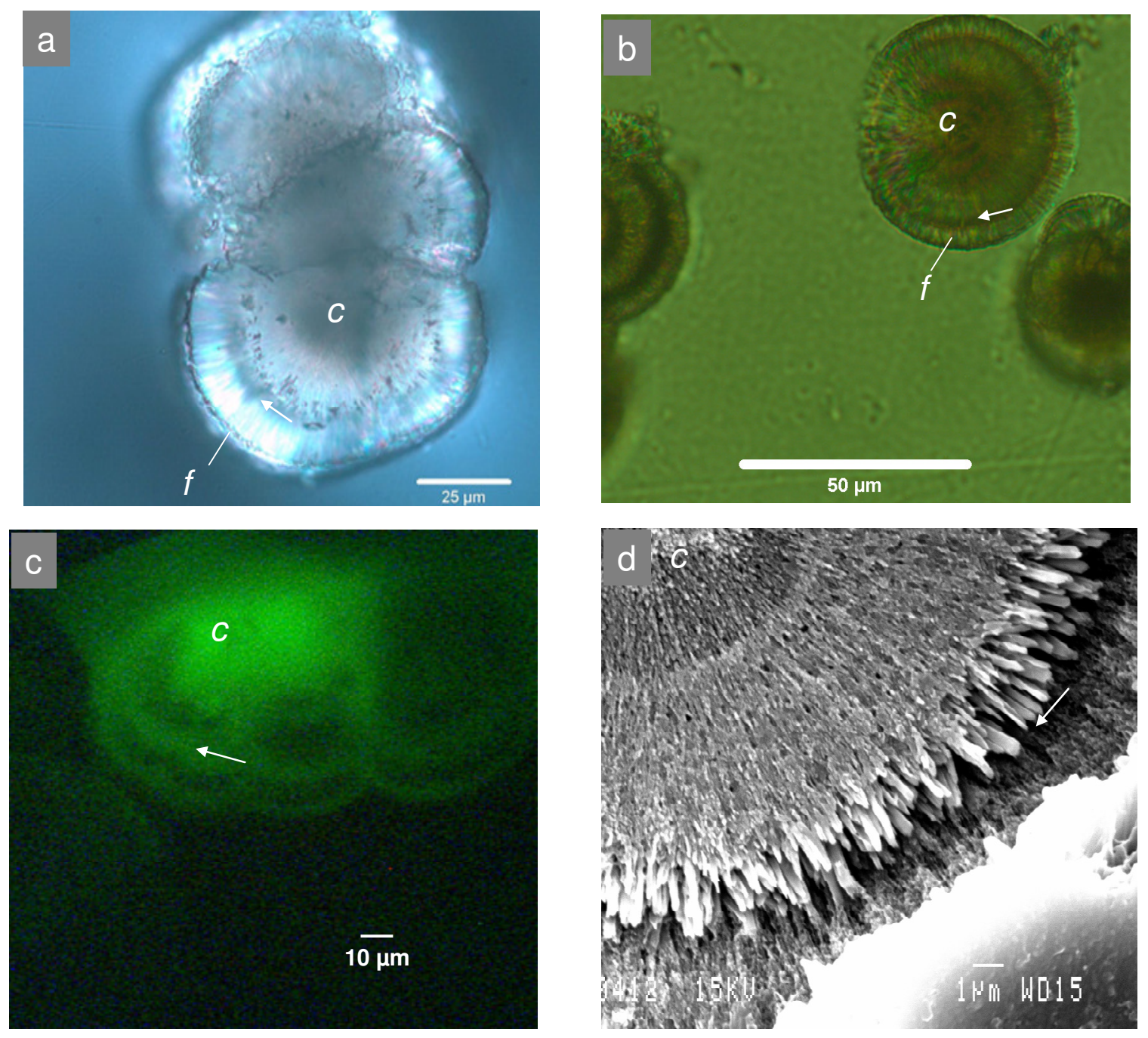

Figure 2. Light microscopy and SEM images of synthetic aragonites formed in

Experiments $2(\mathrm{a}, \mathrm{c})$ and $3(\mathrm{~b}, \mathrm{~d})$. a and b: Transmitted polarized light micrographs of polished sections showing bands (indicated by arrows) perpendicular to the direction of fibrous aragonite $(f)$ growth and centers $(c)$ in synthetic aragonite grains formed in: a. Experiment 2 at $65^{\circ} \mathrm{C}$, and b. Experiment 3. c: Fluorescence microscopy image showing fluorescent bands (arrows) and center $(c)$ in an acridine orange stained synthetic aragonite grown in Experiment 2 at $65^{\circ} \mathrm{C}$. d: SEM image of an synthetic aragonite grain formed in experiment 3 showing a center (c), and a band (arrow) with fibers on either side of the 
band. Specimen was polished and etched with $.1 \mathrm{~N} \mathrm{HCl}$ for $10 \mathrm{~s}$. 

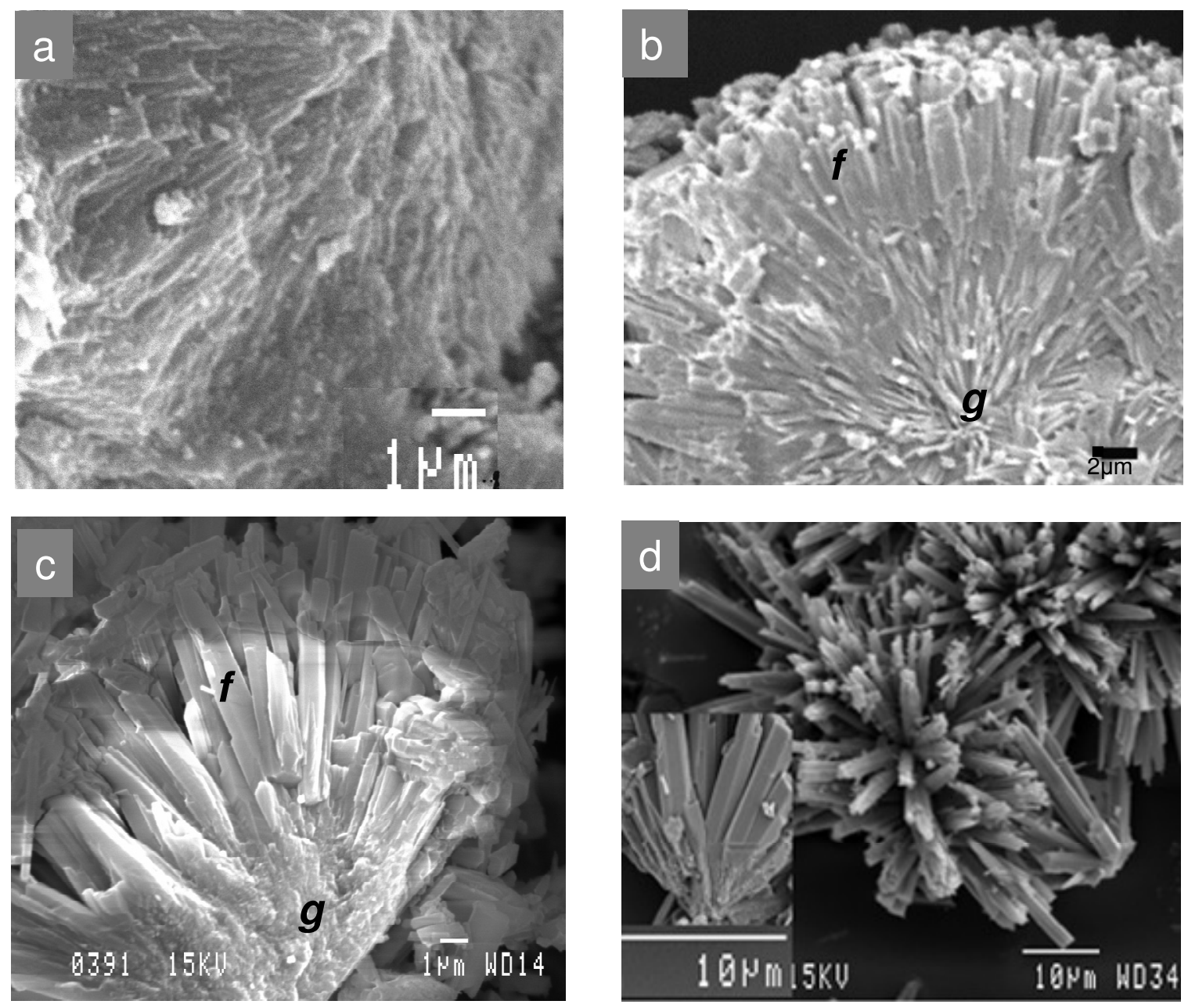

Figure 3. SEM images of synthetic aragonite grains formed in Experiments 4 (a), 5 (b), 7 (c), 6 (d) showing two crystal morphologies - granular $(g)$ and blade like fibers $(f)$. a: Aragonite grain formed at a high pumping rate in Experiment 4 exhibiting a granular to finely fibrous surface. b: Aragonite grain formed at a moderate pumping rate in Experiment 5, exhibiting blade like crystals. c: A low pumping rate grain grown without seed crystals in Experiment 7 showing widely spaced blades arising from a granular center. d: A low pumping rate grain grown with seed crystals in Experiment 6 showing 
widely spaced blades arising from a common center (inset). Accelerating voltages are $6 \mathrm{kV}$ for $\mathrm{b}$ and the inset in $\mathrm{d}$, all others are at $15 \mathrm{kV}$. 

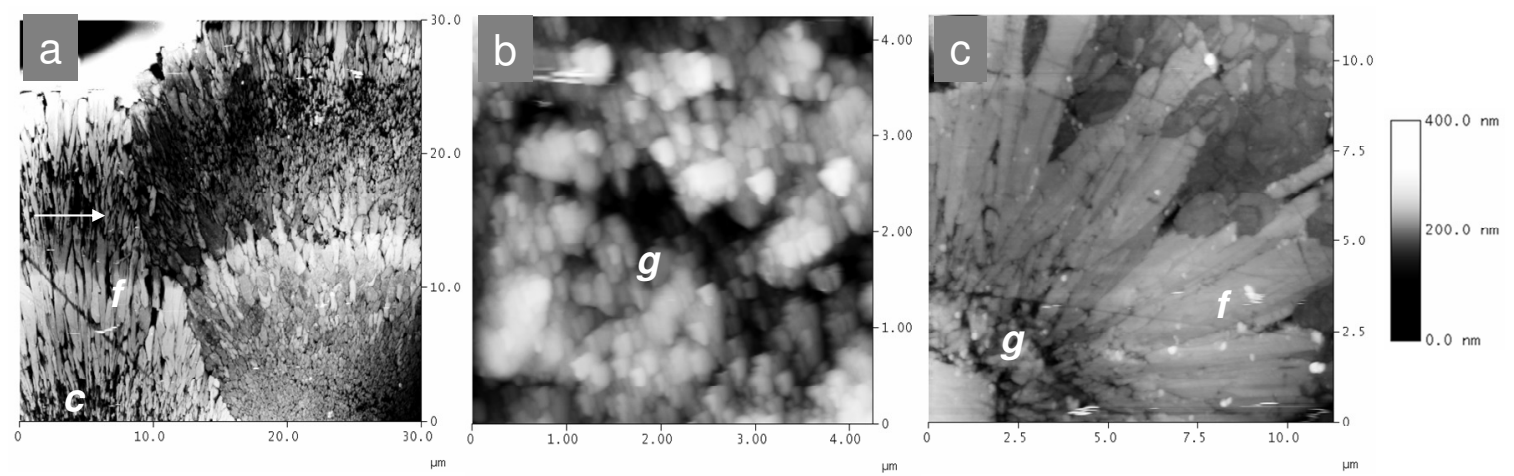

Figure 4. AFM height images of synthetic aragonite grains from Experiment 2 (a), 4 (b), and 6 (c) showing centers $(c)$, dark bands (arrows), granular $(g)$ and fibrous aragonite $(f)$. a: banded synthetic aragonite formed in experiment 2. b: synthetic aragonite precipitated in experiment 4 at high pumping rate showing a rough, granular surface. c: synthetic aragonite precipitated at low pumping rate (Exp. 6) showing blade like fibers $(f)$ and a granular center $(g)$. All specimens were polished with colloidal silica. The height scale (z-axis) is shown on the right. 


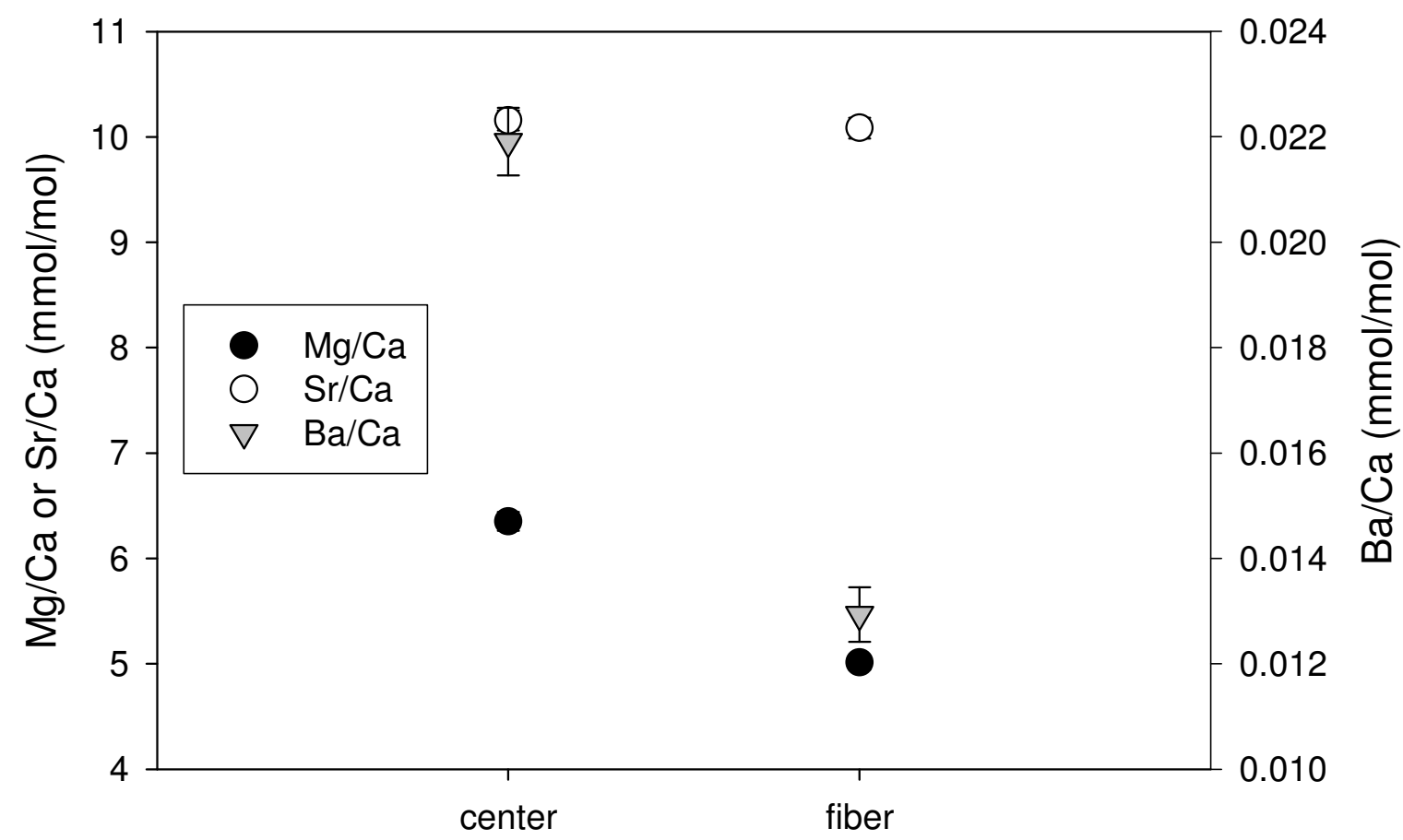

Figure 5. M/Ca ratios determined with SIMS at centers and in fibers of synthetic aragonite grains formed in experiment $1 . \mathrm{Mg} / \mathrm{Ca}$ and $\mathrm{Ba} / \mathrm{Ca}$ ratios are higher at centers than in adjacent fibers $(\mathrm{p}<0.01) . \mathrm{Sr} / \mathrm{Ca}$ ratios show no significant difference with respect to location. Symbols represent means of 4 different grains ( 3 for Ba), error bars represent 1 standard error and may be smaller than the symbol size. 

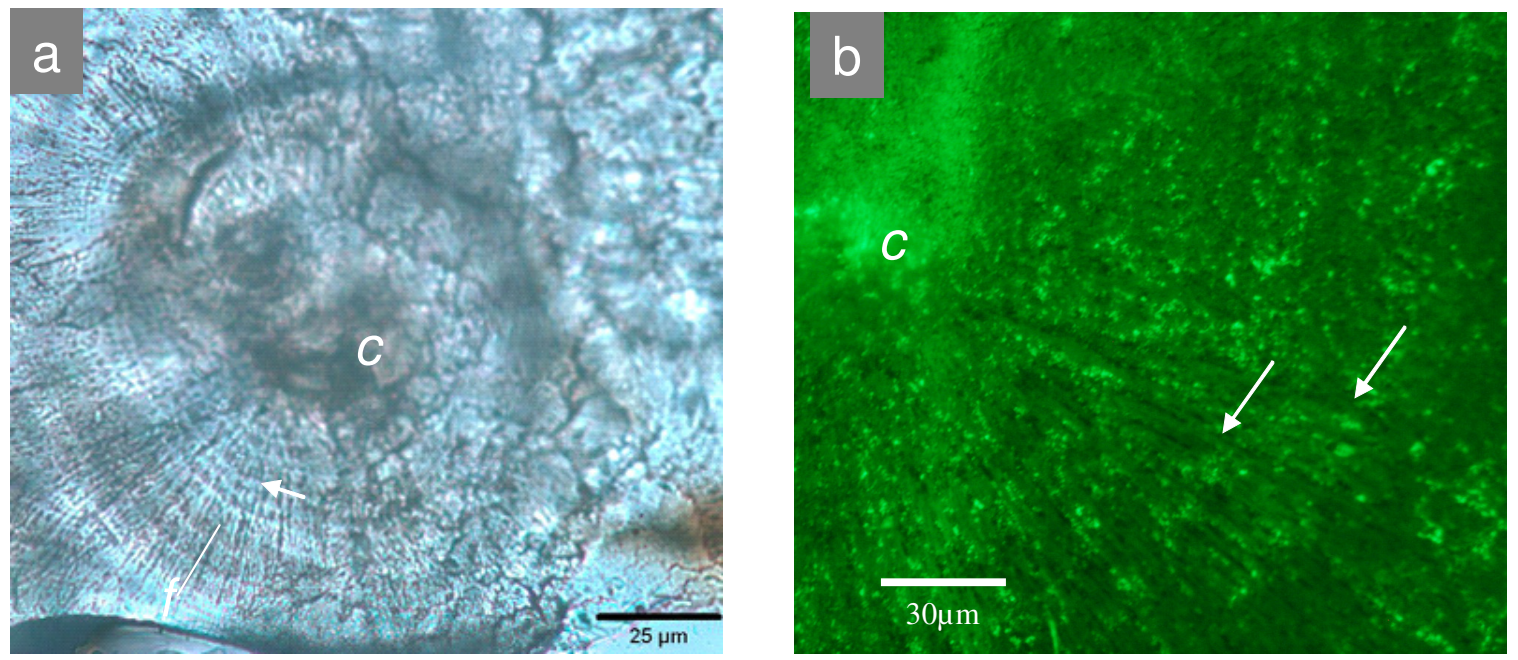

Figure 6. Light microscopy images of corals. a: Transmitted polarized light micrograph of polished section showing bands (indicated by arrows) perpendicular to the direction of fibrous aragonite $(f)$ growth and center of calcification $(c)$ in Porites lutea. b:

Fluorescence microscopy image showing fluorescent bands (arrows) and center $(c)$ in an acridine orange stained Diploria labyrinthiformis specimen. 

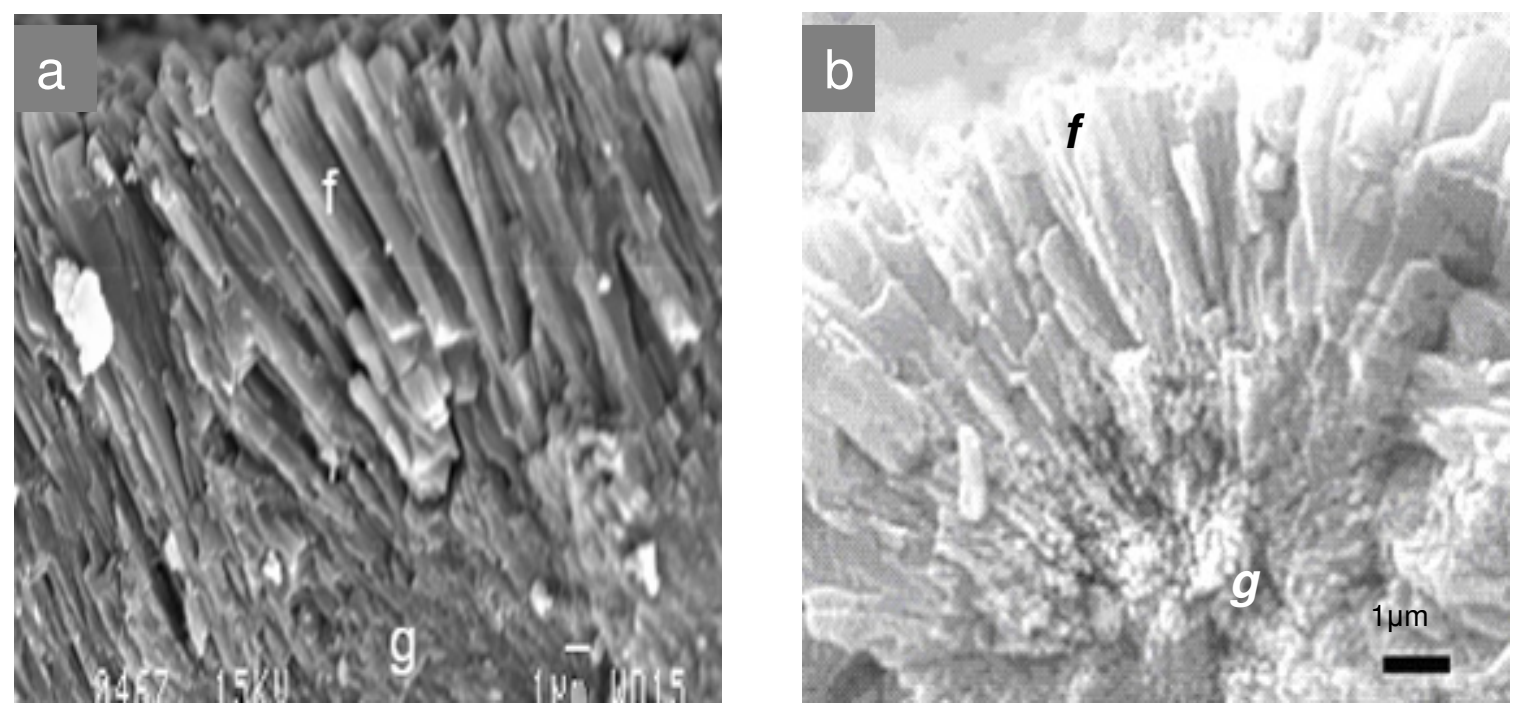

Figure 7. SEM images showing two crystal morphologies in coral dissepiments granular $(g)$ and blade like fibers $(f)$ in a: Diploria labyrinthiformis, b: Porites lutea. 

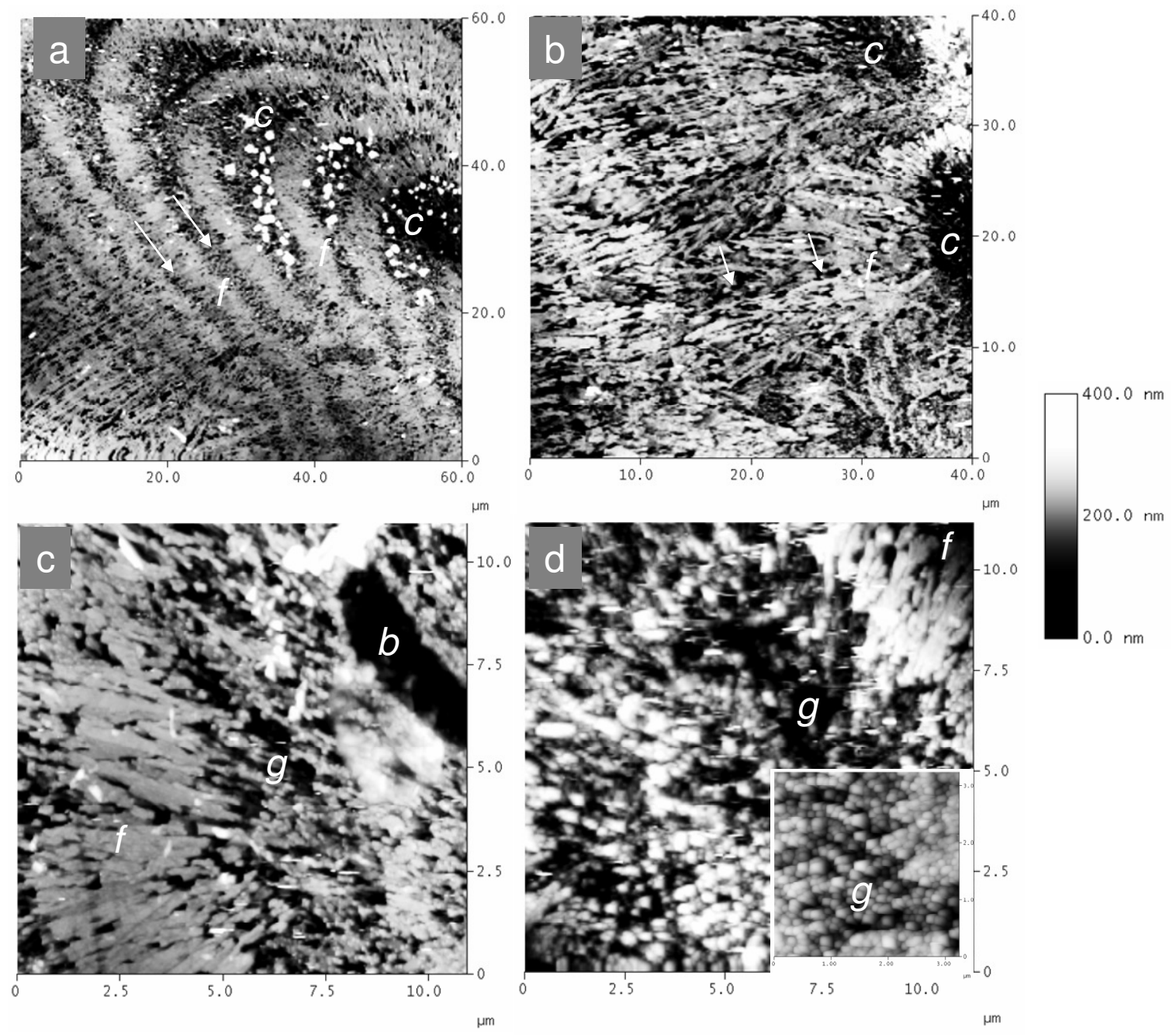

Figure 8. AFM height images of corals centers $(c)$, dark bands (arrows), and fibrous aragonite $(f)$. a: Diploria labyrinthiformis septotheca showing a banded region of the skeleton prepared with an acid etch, note the transition from complete separation of fibers by dark bands near centers, to fibers cutting across bands near the edges. b: Porites solida showing centers of calcification and banded growth. c: Magnified view of Diploria showing a granular band $(g)$, a fibrous band $(f)$ and a boring $(b)$. d: magnified view of Porites solida center with higher magnification inset. Specimen preparation was as follows: for a and c, final polish was with $0.05 \mu \mathrm{m}$ alumina on a microcloth, followed by 
etching $50 \mathrm{~s}$ with .1\% formic acid, $3 \%$ gluteraldehyde, etching stopped by rinsing with water; for b and d, a 30 s etch was used. 


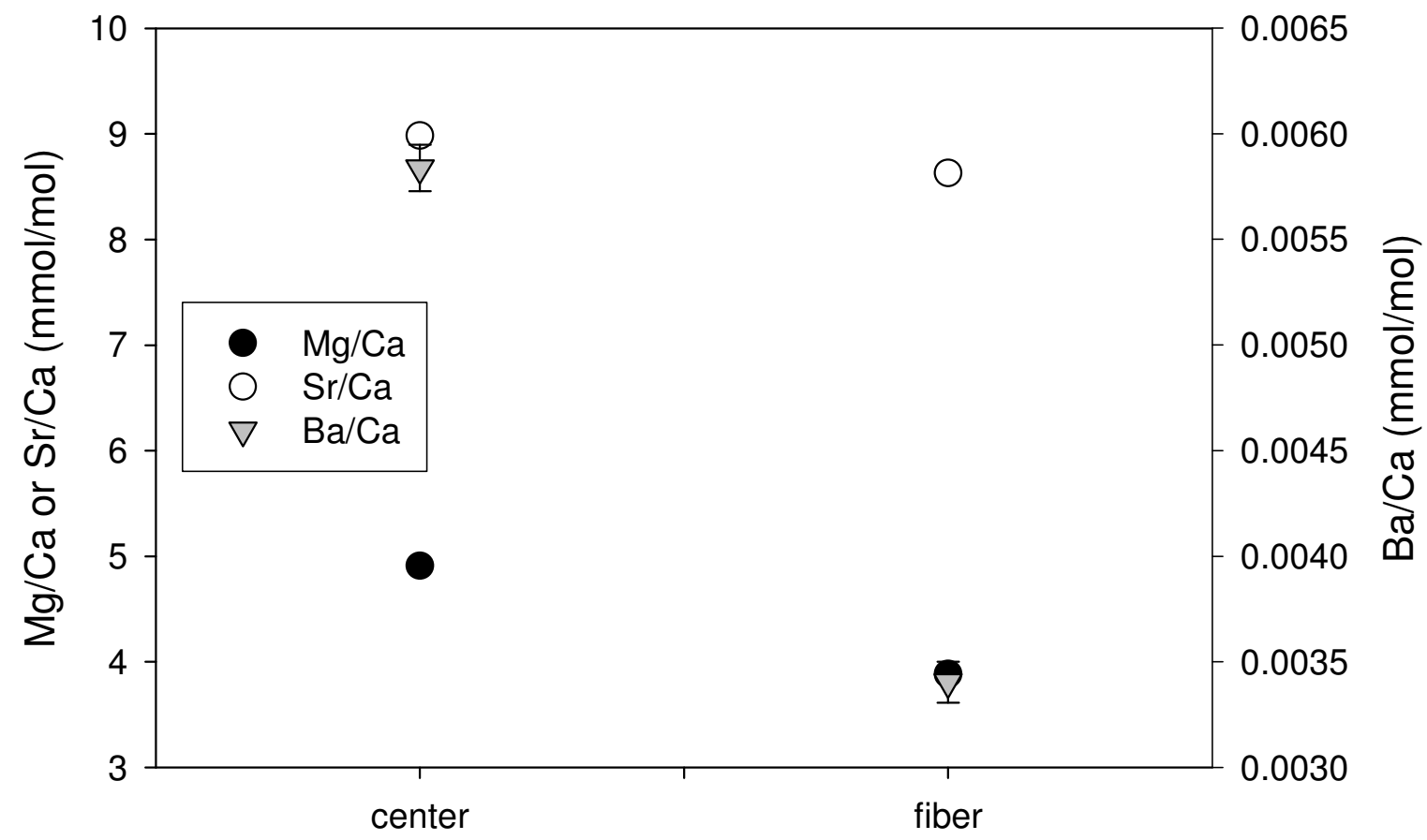

Figure 9. M/Ca ratios measured near centers and in adjacent fibrous aragonite of Porites lutea. $\mathrm{Mg} / \mathrm{Ca}, \mathrm{Sr} / \mathrm{Ca}$, and $\mathrm{Ba} / \mathrm{Ca}$ ratios are higher at centers than in adjacent fibers $(\mathrm{p}<0.01)$. Symbols represent the average of measurements taken at 4 different locations within a single specimen; error bars represent 1 standard error. 


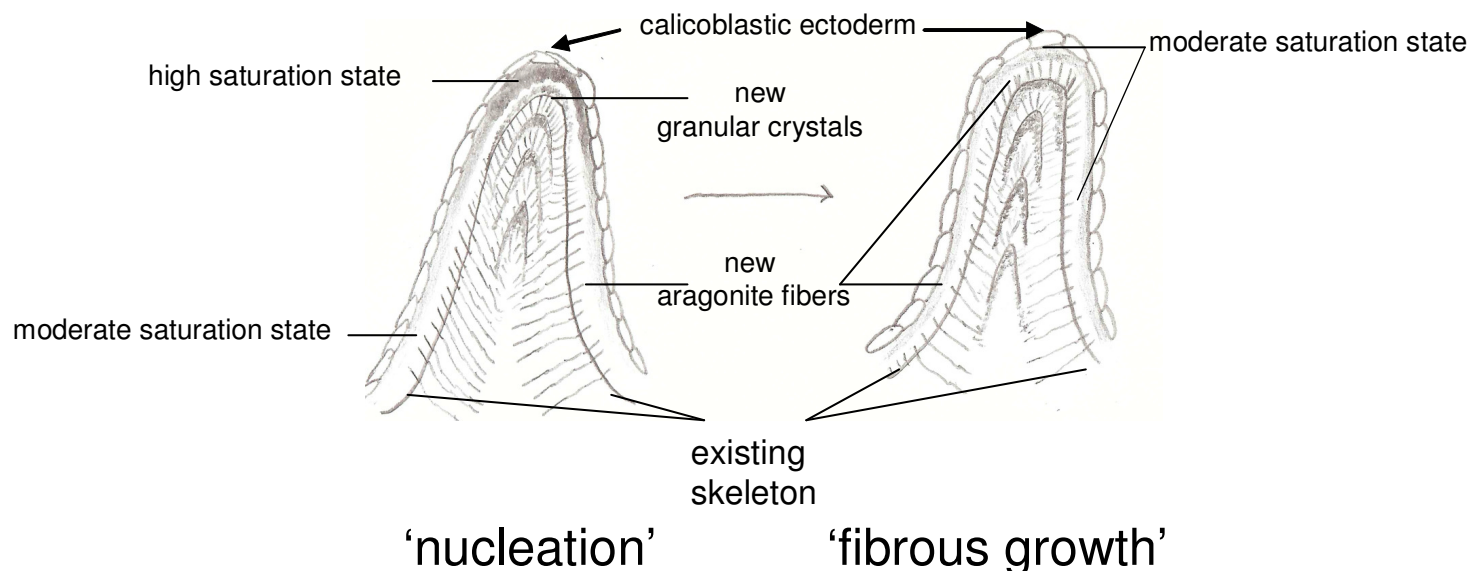

Figure 10. Schematic representation of the cycle in saturation state of the calcifying 'fluid', and the crystals formed in Diploria labyrinthiformis. During 'nucleation' periods, high saturation state adjacent to nascent centers leads to the formation of granular materials at centers. Moving away from centers, the saturation state drops, leading to a transition from the high saturation state granular materials to the lower saturation state fibrous crystals. Between 'nucleation' periods, fibrous growth occurs, during which the calcifying 'fluid' experiences a more uniform, moderate saturation state, and the crystals formed throughout the skeleton are fibrous. 
Chapter 2: Timing of daily growth band formation in coral skeletons

\begin{abstract}
Two distinct types of crystals, sub-micron granular crystals and elongate fibrous crystals are found in the skeletons of scleractinian corals. In thin sections of coral skeletons viewed in transmitted light, granular regions appear dark, while fibrous regions appear light. In many corals these crystal types form alternating light and dark bands, the spacing of which is consistent with a daily cycle of formation. In this study, alizarin, alizarin complexone, calcein, and oxytetracycline were used to mark coral skeletons on day/night cycles. Staining patterns suggest that so-called 'daily' growth bands are indeed formed on a daily cycle, with the dark (granular) bands forming between dusk and dawn. Inorganic precipitation experiments suggest that granular crystals form at a higher saturation state than fibrous aragonite crystals. To generate high saturation states in the evening, a model is proposed in which, for a buffered calcification environment, daytime photosynthetic uptake of $\mathrm{CO}_{2}$ limits the availability of $\mathrm{CO}_{2}$ for calcification, leading to the formation of fibrous crystals during the day. Near dusk, photosynthetic demand for $\mathrm{CO}_{2}$ declines and $\mathrm{CO}_{2}$ ceases to be limiting, allowing a temporary increase in saturation state to occur leading to the formation of dark bands.
\end{abstract}




\section{Introduction}

Rising atmospheric $\mathrm{CO}_{2}$ levels are changing the carbonate chemistry of the oceans. As the ocean becomes more acidic over the next century, the potential negative impact on coral calcification could result in the loss of coral reefs in many parts of the world (e.g., Kleypas et al., 1999). Most experimental evidence indicates that coral calcification is negatively impacted by elevated $\mathrm{pCO}_{2}$ (e.g., Langdon and Atkinson 2005). However, the mechanism(s) by which ocean acidification impacts coral calcification is not clear. Though rising $\mathrm{pCO}_{2}$ reduces the aragonite saturation state of the seawater in which corals grow, it is not clear that this should significantly affect coral calcification given the degree to which corals are thought to elevate saturation state at the site of calcification relative to the external saturation state (Al-Horani et al., 2003).

Coral calcification occurs via the nucleation and growth of aragonite crystals (Cohen and McConnaughey 2003). Factors affecting either the nucleation or the growth step could adversely affect coral skeletal growth. Upward extension of the skeleton is linked with the nucleation phase of crystal growth based on centers of calcification being primarily aligned with the vertical axis of skeletal growth, whereas fibrous crystal growth occurs at an angle of $\sim 20^{\circ}$ to the axis of extension, thus accounting for thickening of skeletal elements and the bulk of calcification (Cohen et al., 2001). Based on diel observations of skeletal growth, skeletal extension and skeletal thickening have been suggested to occur during different times of the day, with extension dominating at night, and thickening dominating during the day (Barnes and Crossland 1980; Vago et al., 1997; 
Cohen and McConnaughey 2003). By implication, crystal nucleation would occur mainly at night while fibrous crystal growth would occur mainly during the day.

Al-Horani et al. (2003) measured the $\mathrm{pH}$ near the site of calcification over lightdark cycles. They found that the $\mathrm{pH}$ of the calcifying fluid increased from near ambient values to above 9 in the light, but dropped rapidly back to near ambient values $(\sim 8)$ in the dark. Based on the saturation state estimates of Al-Horani et al. (2003) (Table 1), and the calcification timing estimates noted above, nucleation (skeletal extension) should be occurring at low saturation state $(\Omega \sim 3.2)$, while growth (thickening) occurs at high saturation state $(\Omega \sim 25)$. However, such a pattern does not agree with saturation state estimates based on crystal morphology (Holcomb et al., 2009).

Crystals accreted by the coral during the nucleation phase are morphologically distinct from crystals accreted during the growth phase. Nuclei are submicron in size and granular in shape. They are found in centers of calcification and in micron-scale bands which transect (cut across) fibrous crystals and appear dark in transmitted light. Conversely, crystals accreted during the growth phase can be several microns long and up to $2 \mu \mathrm{m}$ in diameter. These crystals make up the bulk of the coral skeleton. In inorganic precipitation experiments, long, thin aragonite crystals grow when the seawater saturation state is moderately elevated above that of ambient seawater $(\Omega \sim 5-15)$, while granular material forms when seawater saturation states are significantly elevated above ambient seawater values $(\Omega>20)$.

Using these observations of inorganic aragonite crystal growth to interpret growth of coral crystals implies that crystals accreted by the coral during the nucleation phase, 
crystals thought to be accreted primarily at night, are grown when the saturation state of the calcifying environment is high. Conversely, crystals accreted by the coral in the day are morphologically similar to abiogenic aragonites accreted from a moderately supersaturated fluid. This pattern is inconsistent with predictions based on the saturation state estimates of Al-Horani et al. (2003) (Table 1).

In this study, alizarin, alizarin complexone, calcein, and oxytetracycline were used to mark the skeletons of living corals over the diel cycle in an effort to identify the time of formation of granular versus fibrous crystals and establish their potential relationship to saturation state. A model is then presented to integrate the findings of this study with existing studies.

\section{Methods}

\section{Collection and staining}

Fragments (ranging in size from $\sim 0.13 \mathrm{~cm}^{3}$ to $\sim 10 \mathrm{~cm}^{3}$ ) of Porites lutea. and Goniastrea edwardsi were collected from 3m depth on October 30, 2007 from a back reef environment adjacent to Lizard Island Research Station (Great Barrier Reef, Australia). Fragments were returned to the Lizard Island Research Station, attached to $\sim 2.5$ x $5 \mathrm{~cm}$ acrylic slides using cyanoacrylate adhesive and placed in 1 L PET (SOLO) containers held within an outdoor flow through seawater tank beneath $\sim 50 \%$ shade cloth. Unfiltered water pumped from near the site of collection was continuously supplied to the tank to maintain temperature and nutrient conditions similar to those naturally 
experienced. Air was bubbled in each container using aquarium air-stones to provide circulation.

Following collection, corals were allowed to recover for several weeks. After the recovery period, corals were periodically isolated by raising the $1 \mathrm{~L}$ container holding a given coral fragment above the water level of the tank. Aeration was continued at normal rates. This ensured that coral was minimally disturbed prior to dye addition. Containers were covered with PET lids to reduce evaporation during the incubation. Once isolated, one of four dyes was added to each container to mark the skeleton: alizarin red S (sodium salt), alizarin complexone, calcein, and/or oxytetracycline (buffered with $\mathrm{NaHCO}_{3}$ ). Alizarin red $\mathrm{S}$ has been widely used for marking coral skeletons (e.g., Barnes 1972), while the remaining dyes have been used extensively for marking fish otoliths (e.g., Thorrold et al., 2002). Preliminary experiments conducted at the Woods Hole Oceanographic Institution (Woods Hole, MA USA) with the coral Astrangia poculata were used to establish dye concentrations which gave detectable marks in the coral skeleton but did not appear to adversely affect the health of the coral (data not shown). Dye incubations were ended by re-submerging the container. A total of eight $P$. lutea and six G. edwardsi fragments were stained. No more than two were stained on a given night and each was used for no more than three dye incubations. The number of dye additions a given coral was exposed to was kept low to minimize stress to the coral, as some dyes are known to adversely affect calcification (e.g. Dodge et al., 1984). How long it takes for a given dye to reach the site of calcification, and once there to be flushed from the system is unknown, however two dyes, alizarin red $\mathrm{S}$ and tetracycline have been 
used in previous work on relatively short time scales (Barnes 1972; Lamberts 1974; Gladfelter 1983; Raz-Bahat et al., 2006). Dye uptake is expected to be rapid based on studies in which staining was carried out over three or fewer hours (Barnes 1972; RazBahat et al., 2006). Retention by the tissue may prolong the effective staining duration as suggested by relatively broad stain-lines found by Barnes (1972) despite short incubations, and by the observations of transport of dye within the coral Gladfelter (1983). Despite such limitations, incorporation of alizarin has been found to be tightly correlated with calcium incorporation (Lamberts 1974). Based on these observations, dye incorporation is expected to provide a relatively low resolution time marker, thus $12 \mathrm{hr}$ dye incubations were used in an effort to differentiate day versus night deposition. Incubation intervals and dye concentrations are specified in figure captions and in Table 2. Light levels and temperature were recorded using Pendant loggers (Onset Inc.) placed in the holding tank at approximately the same depth as the corals with the light sensor facing up.

\section{Specimen preparation}

Sections were cut from each specimen roughly parallel to the growth axis using a low speed saw (Buehler) and mounted inside $2.5 \mathrm{~cm}$ diameter $\mathrm{Al}$ rings using Epo-thin epoxy (Buehler). Mounts were ground on $\mathrm{SiC}$ sandpaper using an automatic polisher (Minimet 1000, Buehler) followed by polishing with alumina grit on nylon cloths (Mark V laboratories); final grit size was $1 \mu \mathrm{m}$, grits were suspended either in water or mineral oil. Polishing times on a given grit size were highly variable, ranging from 8 min to several hours, when a specimen was deemed to have a satisfactory polish for a given grit 
size based on visual inspection, it was cleaned prior to polishing with the next smaller grit size. Between grit sizes, water polished specimens were rinsed clean with water; oil polished specimens were wiped with a paper towel. Once polished, the polished face was attached to a $2.5 \mathrm{~cm}$ diameter glass slide using either Epo-thin epoxy or hot melt glue (Crystal bond); most sections were attached using Epo-thin epoxy to reduce the risk of thermal degradation of the dyes. Most of the Al ring mount was cut off using a low speed saw and the remaining portion on the slide polished until the section was thin enough for transmitted light microcopy.

Imaging

Transmitted light microscopy for imaging alizarin and alizarin complexone stained samples was performed on a Nikon Eclipse E 600 Polarizing microscope equipped with a Spot Insight color CCD camera. Fluorescence microscopy for imaging calcein and oxytetracycline stained specimens was performed on a Zeiss Axio Imager Z1 microscope with an Axiocam HR camera at the Marine Biological Laboratory Center for Microscopy. Calcein images were taken using a Zeiss 38HE filter set, oxytetracycline images were taken using filter set 2. Images were aligned using Adobe Photoshop CS3 borings, and other physical structures, visible in both light and fluorescence images were aligned such that all structures visible in both images overlapped. Only images which had structures which could be aligned at multiple positions across the image were used to establish the relationship between stain lines and light vs. dark bands. For overlay images, the color scale was inverted for the fluorescence image so that stained regions 
appear dark, and image transparency set to $40 \%$. Atomic force microscope (AFM) images of Diploria were generated in the laboratory of Jeffrey Hutter at the University of Western Ontario, details on the specimen, preparation and imaging are given in Holcomb et al. (2009).

\section{Results}

The daily temperature (Fig. 1a,1b) and light cycle (Fig. 1c,1d) is displayed in Figure 1 for the time at which each coral was spiked.

The alternating light and dark bands seen in thin sections of coral skeletons and the corresponding crystal morphology are illustrated in Figure 2. Transmitted light microscopy reveals alternating light and dark bands in the skeleton of G. edwardsi (Fig. 2a). Banding is not visible throughout the skeleton, partly due to specimen preparation regions that are not cut parallel to the axis of fiber growth or have not been sufficiently polished often do not show bands. However, band-free regions may also be due to areas composed of uninterrupted fibers without any bands, as has previously been shown with AFM imaging (Holcomb et al., 2009). AFM imaging of Diploria reveals alternating bands of high (light) and low (dark) relief (Fig. 2b). The higher regions are composed of elongate (fibrous) aragonite crystals and correspond to light bands seen with light microscopy. Regions of lower relief are composed of fine to granular crystals and correspond to dark bands seen in light microscopy. The bands are not uniform; in Diploria, dark bands are generally well defined near centers of calcification, while far 
from centers, large regions of fibrous aragonite are often seen without any dark bands interrupting the fibers.

Staining with alizarin, calcein, and oxytetracycline all gave clear stain lines readily distinguished from unstained skeletal features. Samples stained with alizarin complexone had faint stain lines which were difficult to distinguish from unstained regions (data not shown). Results were similar for oil and water polished specimens, but dye intensity tended to be greater in oil polished specimens. All night time stains incorporated in banded regions correspond to dark bands, often, but not always, separated by a light band. Daytime stains tended to correspond to light bands. Daytime staining was difficult to detect, in part due to alizarin and alizarin complexone being the only dyes used for daytime staining, with alizarin being used at a lower concentration than previously used for nighttime stains (alizarin concentrations were reduced due to signs of stress at the concentration originally used - see appendix).

Coral specimens stained on two successive nights are shown in Figures 2a, 3-6. G. edwardsi (Fig. 2a) and P. lutea (Fig. 3) stained with alizarin on two successive nights shows red (alizarin) staining associated with two dark bands. G. edwardsi stained with calcein (Fig. 4b) or oxytetracycline (Fig. 5b) shows fluorescence associated with bands which appear dark in transmitted light (Fig. 4a, 5a). In all cases stain lines are separated by an unstained light band as can be seen in the overlay images (Fig. 4c, 5c).

Staining was not always associated with visible light-dark bands. A different region of the same G. edwardsi specimen stained with calcein on two successive nights shown in Figure 4 is shown in Figure 6. In some regions, only a single stain line is 
visible, in others two stain lines are present, and the stained regions appear to be interrupted by an unstained region (Fig. 6b). Stain lines appear to correspond to a skeletal region in which no light-dark bands are visible in transmitted light (Fig. 6a).

Daytime staining of light bands was also observed. In Figure 7, a specimen of $P$. lutea stained with calcein at night, alizarin during the day, and alizarin complexone on the following night is shown. A red band indicative of alizarin staining corresponds to a light band as seen in transmitted light (Fig. 7a). Fluorescence imaging shows calcein staining (Fig. 7b), potentially associated with the preceding dark band (Fig. 7c). However, only a single feature could be aligned in both the transmitted light and fluorescence microscopy images, so assignment of the fluorescent band is uncertain.

\section{Discussion}

In banded regions of the coral skeleton, all four dyes suggest nighttime deposition of dark bands. Since multiple dyes on multiple nights all show a similar pattern of dye incorporation into dark bands at night it seems likely that dark bands are deposited between dusk and dawn, as opposed to being an artifact from dye exposure. Stain lines deposited on successive nights were generally separated by a light unstained band. Thus, staining patterns support a daily cycle of band formation. This result confirms the report of Sandeman (2008), who used alizarin staining to determine that 'daily' bands in Agaricia agaricites were formed on a daily basis, but is contrary to his conclusion that the dark bands form during the day. The apparent difference in timing of dark band formation could reflect a difference in the timing of band formation due to local 
environmental factors or species specific differences. However it is also possible that the bands were laid down at the same time in both studies. In the current study, all stains were added either between dusk and dawn or between dawn and dusk, so features formed in the morning or the evening could be stained by both daytime and nighttime dye exposures. If dark bands were formed near dusk, then a stain line added a few hours after dusk might not stain the dark band and lead to the conclusion that light bands form at night. Similarly, a daytime stain left late enough in the day could stain the dark band and lead to the conclusion that dark bands formed during the day. Unfortunately, no details were included as to the timing nor means of detection of alizarin spikes in the report by Sandeman (2008), thus it is not possible to evaluate whether the results of the current study differ from those of Sandeman (2008), or if the apparent difference could reflect the time at which dye spikes were present.

Since 'daily' coral growth bands appear to be formed on a daily cycle, they suggest that physiological or environmental processes occurring on a daily basis lead to daily variations in skeletal growth. In zooxanthellate corals, calcification is known to occur at higher rates during the day than at night due to photosynthesis (e.g., Gattuso et al., 1999), while skeletal extension has been shown to increase during the evening (Barnes and Crossland 1980; Vago et al., 1997). Both photosynthesis and calcification reach a maximum during the day and a minimum at night, and thus could be linked to daily banding. However, if photosynthesis controls band formation, dark bands should form either during the day or during the night, but not at the transition between day and night, so there should not be any ambiguity associated with day/night stains. 
However, if extension (which occurs mostly during the evening (Barnes and Crossland 1980; Vago et al., 1997)) is linked to band formation, it could allow both daytime and nighttime stains to mark skeleton formed during the period of maximal extension. Both dark bands and centers of calcification have been shown to share a granular crystal morphology, and may even be continuous structures (Holcomb et al. 2009), thus they may be deposited at the same time. Since deposition of new centers of calcification extends the skeleton (Cohen et al., 2001), and skeletal extension is greatest in the evening (Barnes and Crossland 1980; Vago et al., 1997), dark bands may be expected to form in the evening as well. Thus, formation of dark bands in the evening is consistent with patterns of skeletal extension, and formation at the end of the day to early in the night could allow for staining patterns to suggest both daytime (Sandeman 2008) and nighttime (current study) formation of dark bands depending upon the interval of dye exposure.

\section{Staining irregularities}

Though staining on successive nights generally gave pairs of stain lines, in some instances only a single stain line was found (Fig. 6). Also staining was not uniform throughout a specimen: some septa were stained while adjacent septa were not, and even within a given skeletal element, stain lines could be interrupted by unstained regions. Such patterns may indicate that the coral is able to exclude dye from certain regions, or that coral growth is not uniform, as has previously been suggested by Gladfelter et al., (1983). Isotope based techniques have similarly shown heterogeneity in skeletal growth, 
with regions of active growth changing over time (Marshall and Wright 1998; Al-Horani et al., 2005; Houlbreque et al., 2008), suggesting that dye based methods may reflect real variations in skeletal growth.

\section{Mechanism for band formation}

There are numerous mechanisms which could account for the formation of dark bands. If corals produce organic materials which catalyze nucleation, inhibit growth of fibers, or otherwise alter crystal morphology, then spatial or temporal variations in organic matter composition could result in banding. If zooxanthellae take up compounds which inhibit crystal growth while carrying out photosynthesis, a decline in photosynthesis in the evening could allow more of these compounds to reach the site of calcification. Nighttime tissue expansion could increase rates of fluid movement through the calcifying environment, decreasing diffusion distances and thus increasing the rate at which ions reach the skeleton. Though all of these processes could play a role in determining crystal morphology, here the relationship between saturation state and the evening decline in photosynthesis will be examined as a potential mechanism controlling crystal morphology since both the interaction of photosynthesis with calcification and the daily cycle in photosynthesis are well established. The primary focus will be on the effects of $\mathrm{pCO}_{2}$, though changes in rates of ion pumping are also likely to be involved.

The effect of $\mathrm{CO}_{2}$ removal or influx will first be modeled for seawater (buffered primarily by carbonate and borate) to illustrate the potential for cycles in $\mathrm{pCO}_{2}$ to explain the observed calcification patterns. If photosynthesis could hold internal $\mathrm{pCO}_{2}$ near-zero 
during the day, then in principle it could generate the inferred range of saturation state at the site of calcification with the right periodicity to generate dark bands in the evening, potentially consistent with both the current study and the work of Sandeman (2008). However, this scenario will be dismissed as being physiologically unreasonable as the $\mathrm{pCO}_{2}$ depletion required to generate and maintain the expected saturation states seems unrealistic using seawater. It will then be proposed that since the calcifying environment is in close contact with the tissue and contains organic molecules it should not be modeled as pure seawater, and some of the additional molecules present will be assumed to serve as buffers. The behavior of this fluid will be modeled as if it were seawater containing ammonia (in reality the composition would be substantially different, and activity coefficients may deviate from those associated with seawater, however the simplified model is useful for illustrating how a buffer can affect the behavior of the system). It will be shown that by buffering the calcifying environment, a fluid behavior consistent with $\mathrm{pH}$ measurements, saturation state estimates, and timing estimates can be generated under more realistic conditions. Both models will consider only fluid behavior as $\mathrm{pCO}_{2}$ changes, calcification and alkalinity production will not be considered, however the potential effect of calcification and alkalinity production will be briefly discussed.

\section{Model requirements}

Existing data, in the form of crystal morphologies (Holcomb et al., 2009), pH measurements (Al-Horani et al., 2003) and extension rate data (e.g. Vago et al., 1997) provide constraints on both the likely conditions within the calcifying environment at the 
time of band formation and the diel cycle in solution chemistry within the coral. Holcomb et al. (2009) used crystal morphology and composition to show that granular crystals, similar to those found in dark bands in coral skeletons, are associated with precipitation from a highly super-saturated fluid $(\Omega>\sim 20)$ in inorganic aragonite precipitation experiments. At a saturation state of $\sim 10$, inorganic experiments produced aragonite fibers with morphologies similar to those found in corals. Thus, the work of Holcomb et al. (2009) suggests that fibrous aragonite forms when the site of calcification within the coral is near $\Omega=10$, while the formation of granular crystals associated with dark bands and centers of calcification occurs at $\Omega>\sim 20$. And, if dark band formation occurs in the evening as staining and extension patterns suggest, then the saturation state at the site of calcification is highest in the evening. Thus any mechanism to explain the formation of dark bands and centers of calcification would need to generate a peak saturation state in the evening while maintaining lower saturation states through the rest of the night and much of the day.

However, direct measurements of the calcifying environment suggest a different pattern. Al-Horani et al. (2003) observed that the $\mathrm{pH}$ near the site of calcification declined rapidly in the dark, suggesting that calcification site saturation state could be lower too. But, measurement of $\mathrm{pH}$ alone is not sufficient to constrain saturation state, so although $\mathrm{pH}$ is declining, it is possible for saturation state to increase. To elevate saturation state when $\mathrm{pH}$ is declining, the coral could increase the calcium ion concentration, however this possibility is not supported by the calcium measurements of Al-Horani et al. (2003). Instead of increasing calcium concentrations, the coral could 
elevate saturation state by increasing carbonate ion concentration. Carbonate ion concentrations could be increased by removing protons from the site of calcification, but this alone would increase $\mathrm{pH}$. To allow $\mathrm{pH}$ to decrease while simultaneously elevating saturation state, dissolved inorganic carbon (DIC) must increase. The high $\mathrm{pH}$ values in the light, associated with photosynthesis, may reflect photosynthetic draw down of $\left[\mathrm{CO}_{2}\right]_{\mathrm{aq}}$ which could reduce DIC levels within the coral to the point that DIC limits calcification (e.g. Marubini and Davies 1996). Under this condition, $\mathrm{CO}_{2}$ reaching the site of calcification during the day would be converted to carbonate (due to $\mathrm{pH}>9$ ) and deposited as $\mathrm{CaCO}_{3}$. If $\Omega_{\text {arag }}$ during the day were DIC limited, the DIC increase at dusk could be more important than the $\mathrm{pH}$ decrease, and carbonate ion concentration and $\Omega_{\text {arag }}$ could rise.

The carbon supply for calcification is provided by both DIC in seawater and by respiratory generation of $\mathrm{CO}_{2}$ in the tissue layers overlying the site of calcification (e.g., Furla et al., 2000). In zooxanthellate corals, $\mathrm{CO}_{2}$ from any source other than the aboral tissue layers must pass by the zooxanthellae prior to reaching the calcification site. While carbon fixation is occurring (e.g. daytime), zooxanthellae may limit the supply of $\mathrm{CO}_{2}$ which can reach the site of calcification from seawater and compete with calcification for respiratory $\mathrm{CO}_{2}$ generated within the tissue, potentially keeping the $\mathrm{pCO}_{2}$ within the tissue low.

\section{Seawater model}


For seawater held at constant alkalinity, the effect of $\mathrm{CO}_{2}$ removal from seawater is shown in Figure 8 for two different alkalinity levels. At a given alkalinity, removal of $\mathrm{CO}_{2}$ always increases $\mathrm{pH}$, and initially increases saturation state. However, as $\mathrm{pCO}_{2}$ approaches zero (i.e., for $\mathrm{pH}$ values > 9.5), saturation state declines due to the decline in DIC. Though the general pattern in saturation state needed for influx of $\mathrm{CO}_{2}$ in the evening to generate elevated saturation state can be achieved in seawater, the low levels of $\mathrm{CO}_{2}$ needed to achieve reduced saturation states are unlikely to occur. If seawater, as described by Figure 8 were the fluid from which the coral skeleton were grown, an alkalinity of $\sim 2200 \mu \mathrm{mol} / \mathrm{kg}$ would be sufficient for $\mathrm{CO}_{2}$ removal to generate a saturation state $\sim 20$ needed for granular crystals to form, and further reductions in $\mathrm{pCO}_{2}$ could reduce saturation state to $\sim 10$, needed for fibrous growth (Holcomb et al. 2009). However, at an alkalinity value near $2200 \mu \mathrm{mol} / \mathrm{kg}, \mathrm{CO}_{2}$ removal achieves a saturation state of 20 at a $\mathrm{pH}$ of $\sim 9.5$, and additional $\mathrm{CO}_{2}$ removal leads to a saturation state of 10 at a pH of $\sim 10.3$. To maintain a saturation sate of $20, \mathrm{CO}_{2}$ would have to be maintained at a value far below ambient, which is inconsistent with deposition of granular crystals near dusk when photosynthesis is no longer restricting the $\mathrm{CO}_{2}$ supply. Higher alkalinity values could allow a high saturation state to be achieved at ambient $\mathrm{CO}_{2}$ levels, which would be consistent with nighttime deposition of granular crystals, but, high alkalinity values would require correspondingly higher $\mathrm{pH}(>10.5)$ and lower $\mathrm{CO}_{2}$ levels in the daytime to maintain a saturation state of 10 . However, the highest reported $\mathrm{pH}$ values taken near the site of calcification are 9.3 (Al-Horani et al. 2003). Such a pH is well below that required for band formation to be explained by daytime drawdown and 
evening influx of $\mathrm{CO}_{2}$ to a seawater-like fluid. Thus, for band formation to occur from a seawater like medium, the coral must carry out nucleation and growth at lower saturation states than observed in inorganic experiments, or daytime $\mathrm{pH}$ values must be much higher than current microelectrode-based estimates, or the coral must invest more energy in proton removal in the evening to achieve high alkalinities and saturation states.

\section{Buffer model}

The calcifying environment is in direct contact with coral tissue. A wide range of organic compounds produced by corals are found within the calcifying environment to such an extent that several authors (e.g., Puverel et al., 2005) consider calcification to be controlled by the organic materials within the calcifying environment. Thus the chemistry of any calcifying fluid probably departs substantially from that of seawater. Organic materials make up approximately $1 \%$, by weight, of the coral skeleton (Cuif et al., 2004), using a skeletal density of $2.9 \mathrm{~g} / \mathrm{cm}^{3}$, and assuming the organic material is composed of amino acids with an average molecular weight of 130 , this represents a concentration of $\sim 200 \mu \mathrm{mol}$ amino acids $/ \mathrm{cm}^{3}$. If the skeletal organics were originally present in an equivalent volume of water, this could represent a $200 \mathrm{mM}$ solution. The organic materials incorporated into the skeleton are likely only a small fraction of the total organic material which is associated with the site of calcification. Since many organisms use organic molecules, such as proteins, as part of a buffer system (e.g., Abe et al., 1985), one role of the organic material associated with the site of calcification may be to buffer the calcification environment. Based on the amino acid concentration estimate 
above, if only $1 \%$ of the skeletal organics contributed to the buffering capacity of the calcifying environment, the alkalinity contributed by the organics would be similar to the alkalinity of seawater. For several zooxanthellate corals, just two basic amino acids, Lys and Arg make up $3 \%$ of the amino acids in the skeletal organics (Cuif et al., 1999). In addition to large organic molecules, ammonia and urea are both present at significant concentrations in zooxanthellate corals and have been suggested to be involved in the calcification process (Crossland and Barnes 1974). Ammonia is present at concentrations of $80-100 \mathrm{nmol} / \mathrm{mg}$ protein, urea at $430-480 \mathrm{nmol} / \mathrm{mg}$ protein (Crossland and Barnes 1974), to estimate concentrations, a protein content of $8.4 \mathrm{mg} / \mathrm{cm}^{2}$, dry weight of 22 $\mathrm{mg} / \mathrm{cm}^{2}$ (Porter et al., 1989), and water content of 95\% (Tricas 1989) were used to calculate that on the basis of total water content, ammonia is present at a concentration of $\sim 30 \mu \mathrm{M}$, urea at $\sim 130 \mu \mathrm{M}$. A concentration of $\sim 30 \mu \mathrm{M}$ or even $\sim 130 \mu \mathrm{M}$ is low small relative to the buffering capacity of seawater. However, these estimates assume ammonia and urea are uniformly distributed, which is not the case. Urea and ammonia concentrations associated with the zooxanthellae are known to be much lower than those associated with tissue (Crossland and Barnes 1974), thus the concentrations at the site of calcification may be much higher than estimates based on total water content. Ammonia in particular could play a role in proton transport and would serve as an effective buffer in the $\mathrm{pH}$ 8-10 range. Though there are no good estimates of the buffering capacity or pKa for buffering components associated with the site of calcification, the above calculations show that ammonia alone is likely to be present at concentrations greater than $30 \mu \mathrm{M}$, while basic functional groups associated with proteins, sugars and lipids 
could increase buffering capacity by many mM. For illustrative purposes, a buffered calcifying environment will be modeled as seawater with $5 \mathrm{mmol} / \mathrm{kg}$ ammonia, though the actual buffer composition and activity coefficients are expected to differ substantially. A total alkalinity of $5100 \mu \mathrm{mol} / \mathrm{kg}$ (Fig. 9) can allow $\mathrm{CO}_{2}$ removal to elevate saturation state $(\Omega>20)$ until a $\mathrm{pH}$ of $\sim 9$ is reached, while further removal of $\mathrm{CO}_{2}$ would lower saturation state. By buffering the calcifying environment with ammonia, substantially elevated saturation states can be achieved at ambient $\mathrm{pCO}_{2}$ levels. Removal of $\mathrm{CO}_{2}$ to reach a $\mathrm{pH}$ of $\sim 9.5$ allows a saturation state of 10 to be achieved. Thus, photosynthesis combined with a buffered calcification environment could generate a cycle in saturation state consistent with skeletal extension and banding patterns, and $\mathrm{pH}$ patterns consistent with measured values. Though the actual cycle is likely much more complex, in part due to the daytime production of alkalinity and ion transport processes needed to maintain calcification, the saturation state pattern needed to explain the formation of daily growth bands can be generated by $\mathrm{CO}_{2}$ uptake by the zooxanthellae so long as the calcification site is buffered.

\section{Alkalinity and calcification}

In Figures 8-9, alkalinity has been assumed to remain constant, which would require proton export to balance calcification. Since precipitation rate is related to saturation state (Burton and Walter 1987), the higher the saturation state the higher the calcification rate, and thus the higher the proton pumping rate if alkalinity is to be kept constant. However, calcification and photosynthesis decline in the evening. Alkalinity 
most likely follows a similar pattern, with levels rising during the day and falling at night, linked to photosynthesis. $\mathrm{HCO}_{3}{ }^{-}$is the primary source of $\mathrm{CO}_{2}$ for photosynthesis (e.g. AlMoghrobi et al. 1996). Using the photosynthesis and calcification rate estimates of Furla et al. (2000), if $\mathrm{HCO}_{3}{ }^{-}$were the sole source of $\mathrm{CO}_{2}$ for photosynthesis, the rate of alkalinity production by photosynthesis would be nearly double the rate of alkalinity consumption by calcification during the day. Since calcification may not completely neutralize the alkalinity generated by photosynthesis during the day, excess alkalinity must be exported or internal alkalization could occur. To reduce internal alkalization during the day, the coral could produce basic organic molecules and urea which could then be exported to the site of calcification. These basic molecules could then be neutralized by nighttime calcification, while providing a source of alkalinity to maintain rapid calcification during the early part of the night and thus support skeletal extension. The expected daily cycle in chemistry at the site of calcification and photosynthesis is illustrated in Figure 10. At dawn, $\mathrm{pCO}_{2}$ is high due to respiration, while alkalinity, saturation state, and $\mathrm{pH}$ are all low (near seawater values). With the start of photosynthesis, $\mathrm{pCO}_{2}$ falls, $\mathrm{pH}$ and saturation state rise. Photosynthesis continues to increase as light levels increase in the morning, leading to the accumulation of alkalinity, and eventually drawing $\mathrm{pCO}_{2}$ down to a daytime minimum value which is maintained till light levels (and photosynthesis) decline in the evening. As $\mathrm{pCO}_{2}$ rises, $\mathrm{pH}$ falls, but the daytime buildup of non-carbonate alkalinity allows the saturation state to increase, supporting a temporary increase in calcification. Rapid growth (extension) in the evening 
depletes the internal alkalinity pool and alkalinity, $\mathrm{pH}$, and saturation state return to low nighttime values

Under the proposed model, assuming photosynthesis is not stimulated by elevation of ambient $\mathrm{pCO}_{2}$ and the rate of alkalinity production remains unchanged, a rise in external $\mathrm{pCO}_{2}$ could result in a similar rise in internal $\mathrm{pCO}_{2}$, and thus reduce $\mathrm{pH}$ at the site of calcification. If the calcifying environment were described by Figure 9, and had a normal maximum (daytime) $\mathrm{pH}$ of 9.5 , a reduction to a maximum of $\mathrm{pH} 9$ could lead to nucleation becoming a daytime phenomenon, and still further decreases could eliminate nucleation altogether. Modest increases in $\mathrm{pCO}_{2}$ however could reduce the magnitude of $\mathrm{CO}_{2}$ limitation, increase saturation state, and allow calcification to increase, consistent with reports of increased calcification rates under elevated $\mathrm{pCO}_{2}$ (e.g. Reynaud et al. 2003). However, an increase in calcification in the light (or an increase in photosynthetic utilization of $\mathrm{CO}_{2}$ relative to $\mathrm{HCO}_{3}{ }^{-}$) under elevated $\mathrm{pCO}_{2}$ conditions could reduce internal alkalization which could result in lower rates of skeletal extension, consistent with the findings of De'ath et al. (2009).

\section{Summary}

Calcium binding dyes showed that 'daily' bands in coral skeletons are daily structures, with the dark bands forming between dusk and dawn. However, it is not clear that the dark bands represent nighttime growth. The morphology of crystals in dark bands suggests a relatively high saturation state while low night time growth rates 
suggest a relatively low saturation state. Deposition of dark bands may occur in a relatively short interval near dusk when alkalinity (primarily in the form of organic buffers) generated by daytime photosynthesis is available to neutralize protons, and with the cessation of photosynthesis, $\mathrm{CO}_{2}$ is available to relieve daytime DIC limitation of calcification. This scenario could generate a brief period of elevated saturation state during which nucleation, skeletal extension, and dark band formation occur. 


\section{Acknowledgments}

Many thanks to Anne Hoggett and Lyle Vail for making my work at Lizard Island Research Station possible, to Marianne Pearce, Lance Pearce, Monica Gagliano, Harriet Salomonsen, Adel Heenan, Dan Bayley, and numerous visitors for assistance, advice and camaraderie, Anne Cohen, Scott Gallager, Daniel McCorkle, and Ann Tarrant for advice, to Michelle Scott, Katrina Goudkamp and the Great Barrier Marine Park Authority for permitting assistance, and to Louie Kerr, Glenn Gaetani, Jeffrey Hutter and Karen Casciotti for use of equipment. This work was supported by a Lizard Island Doctoral Fellowship and the WHOI Ocean Life Institute. This material is based upon work supported under a National Science Foundation Graduate Research Fellowship. 
References

Abe, H., Dobson, G.P., Hoeger, U., Parkhouse, W.S., 1985. Role of histidine-related compounds to intracellular buffering in fish skeletal muscle. Am. J. Physiol. Rgulatory Integrative Comp. Physiol 249, 449-454.

Al-Horani, F. A., S. M. Al-Moghrabi and D. de Beer (2003). "The mechanism of calcification and its relation to photosynthesis and respiration in the scleractinian coral Galaxea fascicularis." Marine Biol. 142: 419-426

Al-Horani FA, Ferdelman T, Al-Moghrabi SM, de Beer D (2005) Spatial distribution of calcification and photosynthesis in the scleractinian coral Galaxea fascicularis. Coral Reefs 24:173 - 180

Al-Moghrabi, S., Goiran, C., Allemand, D., Speziale, N.J., J., 1996. Inorganic carbon uptake for photosynthesis by the symbiotic coral/dinoflagellate association II. Mechanisms for bicarbonate uptake. J. Exp. Mar. Biol. Ecol. 199, 227-248.

Barnes DJ (1972) The structure and formation of growth-ridges in scleractinian coral skeletons. Proc. R. Soc. Lond. B 182:331-350.

Barnes, D. J., Crossland, C.J. (1980). "Diurnal and seasonal variations in the growth of a staghorn coral measured by time-lapse photography." Limnol. Oceanography 25: 1113-1117.

Burton, E.A., Walter, L.M., 1987. Relative precipitation rates of aragonite and Mg calcite from seawater: Temperature or carbonate ion control? Geology 15, 111-114.

Cohen AL, Layne GD, Hart SR, Lobel PS (2001) Kinetic control of skeletal Sr/Ca in a symbiotic coral: Implications for the paleotemperature proxy. Paleoceanography 16: $20-26$

Cohen AL, McConnaughey TA (2003) A Geochemical Perspective on Coral Mineralization. In: Biomineralization. Dove PM, Weiner S, deYoreo JJ (Eds) Reviews in Mineralogy and Geochemistry Vol. 54 pp 151-187.

Crossland CJ, Barnes DJ (1974) The role of metabolic nitrogen in coral calcification. Marine Biol. 28: 325-332.

Cuif JP, Dauphin Y, Freiwald A, Gautret P, Zibrowius H (1999) Biochemical markers of zooxanthellae symbiosis in soluble matrices of skeleton of 24 Scleractinia species. Comparative Biochemistry and Physiology. 123A: 269-278.

Cuif JP, Dauphin, Y., Berthet, P., Jegoudez, J. (2004) Associated water and organic compounds in coral skeletons: Quantitative thermogravimetry coupled to infrared absorption spectrometry. G-cubed 5:Q11011

De'ath, G., Lough, J.M., Fabricius, K.E., 2009. Declining Coral Calcification on the Great Barrier Reef. Science 323, 116-119.

Furla P, Galgani I, Durand I, Allemand D (2000) Sources and mechanisms of inorganic carbon transport for coral calcification and photosynthesis. J. Exp. Biol. 203: 3445-3457

Gattuso, J. P., Allemand, D., Frankignoulle, M. (1999). "Photosynthesis and calcification at cellular, organismal and community levels in coral reefs: a review of interactions and control by carbonate chemistry." American Zoologist 39: 160183. 
Gladfelter EH, (1983). Skeletal development in Acropora cervicornis II. diel patterns of calcium carbonate accretion. Coral Reefs 2: 91-100.

Holcomb M, Cohen AL, Gabitov RI, Hutter JL (2009) Compositional and morphological features of aragonite precipitated experimentally from seawater and biogenically by corals. Geochimica et Cosmochimica Acta 73:4166-4179

Houlbreque F, Meibom A, Cuif J-P, Stolarski J, Marrocchi Y, Ferrier-Pages C, DomartCoulon I, Dunbar RB (2008) Strontium-86 labeling experiments show spatially heterogeneous skeletal formation in the scleractinian coral Porites porites. Geophysical Research Letters 36:L04604, doi:04610.01029/02008GL036782

Kleypas, J. A., R. W. Buddemeier, D. Archer, J. P. Gattuso, C. Langdon and B. N. Opdyke (1999). "Geochemical consequences of increased atmospheric carbon dioxide on coral reefs." Science 284: 118-120

Lamberts AE (1974) Measurement of alizarin deposited by coral. Proc. 2nd Int. Coral Reef Sym. 2:241-244

Langdon, C. and M. J. Atkinson (2005). "Effect of elevated pCO2 on photosynthesis and calcification of corals and interactions with seasonal change in temperature/irradiance and nutrient enrichment." J. Geophys. Res. Ocean

Marshall AT, Wright A (1998) Coral calcification: autoradiography of a scleractinian coral Galaxea fascicularis after incubation in $45 \mathrm{Ca}$ and $14 \mathrm{C}$. Coral Reefs $17: 37-$ 47.

Marshall AT, Clode PL, Russell R, Prince K, Stern R (2007) Electron and ion microprobe analysis of calcium distribution and transport in coral tissues. The Journal of Experimental Biology 210:2453-2463

Marubini, F., Davies, P.S., 1996. Nitrate increases zooxanthellae population density and reduces skeletogenesis in corals. Marine Biol. 127, 319-328.

Porter JW, Fitt WK, Spero HJ, Rogers CS, White MW (1989) Bleaching in reef corals: Physiological and stable isotopic responses. PNAS 86: 9342-9346.

Puverel S, Tambutte E, Pereira-Mouries L, Zoccola D, Allemand D, Tambutte S (2005) Soluble organic matrix of two Scleractinian corals: Partial and comparative analysis. Comparative Biochemistry and Physiology B Biochemistry and Molecular Biology 141:480-487

Raz-Bahat M, Erez J, Rinkevich B (2006) In vivo light-microscopic documentation for primary calcification processes in the hermatypic coral Stylophora pistillata. Cell \& Tissue Research 325:361-368

Reynaud S, Leclerq N, Romaine-Lioud S, Ferrier-Pages C, Jaubert J, Gattuso J-P (2003) Interacting effects of $\mathrm{CO} 2$ partial pressure and temperature on photosynthesis and calcification in a scleractinian coral. Global Change Biol. 9: 1660-1668

Sandeman, I. 2008. Daily banding in coral septa. $11^{\text {th }}$ ICRS.

Thorrold, S., Jones, G.P., Hellberg, M.E., Burton, R.S., Swearer, S.E., Neigel, J., Morgan, S.G., Warner, R.R. 2002. Quantifying larval retention and connectivity in marine populations with artificial and natural markers. Bulletin of Marine Science 70s:291-308

Tricas TC (1989) Prey selection by coral-feeding butterflyfishes : strategies to maximize the profit. Env. Biol. Fishes 25:171-185. 
Vago, R., Gill, E., Collingwood, J.C. 1997. Laser measurements of coral growth. Nature. 386: 30-31. 
Table 1. Comparison of potential conditions within the calcifying fluid and the period in which the highest saturation state occurs based on the studies of Al-Horani et al. (2003) and Holcomb et al. (2009). ${ }^{1}$ The saturation state estimates of Al-Horani et al. (2003) are based on calculations which assume total DIC to remain constant at a value similar to ambient seawater. * The timing estimate for the period with the highest saturation state is based on the extension rate measurements of Vago et al. (1997), and extension being linked to the formation of centers of calcification (Cohen et al., 2001).

\begin{tabular}{|l|l|l|l|l|l|}
\hline Study & High $\mathrm{pH}$ & High $\Omega_{\text {Arag }}$ & Period of high $\Omega_{\text {Arag }}$ & Low $\mathrm{pH}$ & Low $\Omega_{\text {Arag }}$ \\
\hline $\begin{array}{l}\text { Al-Horani et } \\
\text { al., 2003 }\end{array}$ & 9.3 & $25^{1}$ & light & $8.1^{1}$ & 3.2 \\
\hline $\begin{array}{l}\text { Holcomb et } \\
\text { al., 2009 }\end{array}$ & na & $>20$ & evening* & na & $\sim 10$ \\
\hline
\end{tabular}


Table 2. Timing of dye spikes, dye, and dye concentration used. Spikes designated by the same group letter were applied to the same corals. Dyes used were: oxytetracycline (OTC), alizarin (Aliz), alizarin complexone (AC), and calcein. The number of corals indicates the total number of $P$. lutea and G. edwardsi specimens used. * only P. lutea specimens stained, no G. edwardsi included

\begin{tabular}{|l|l|l|l|l|l|}
\hline Group & Time in & Time out & $\begin{array}{l}\text { \# corals } \\
\text { stained }\end{array}$ & Dye & $\begin{array}{l}\text { Dye } \\
\text { concentration }\end{array}$ \\
\hline a & $12 / 21 / 0718: 00$ & $12 / 22 / 076: 15$ & 2 & OTC & $40 \mathrm{mg} / \mathrm{L}$ \\
\hline a & $12 / 22 / 0718: 00$ & $12 / 23 / 076: 00$ & 2 & OTC & $40 \mathrm{mg} / \mathrm{L}$ \\
\hline $\mathrm{b}$ & $12 / 23 / 0718: 30$ & $12 / 24 / 076: 15$ & 2 & Aliz & $10 \mathrm{mg} / \mathrm{L}$ \\
\hline $\mathrm{b}$ & $12 / 24 / 0718: 50$ & $12 / 25 / 076: 40$ & 2 & Aliz & $8 \mathrm{mg} / \mathrm{L}$ \\
\hline c & $12 / 26 / 0719: 00$ & $12 / 27 / 077: 00$ & 2 & AC & $10 \mathrm{mg} / \mathrm{L}$ \\
\hline c & $12 / 27 / 0718: 25$ & $12 / 28 / 076: 40$ & 2 & AC & $10 \mathrm{mg} / \mathrm{L}$ \\
\hline d & $12 / 26 / 0719: 10$ & $12 / 27 / 077: 00$ & 2 & calcein & $10 \mathrm{mg} / \mathrm{L}$ \\
\hline d & $12 / 27 / 0718: 25$ & $12 / 28 / 076: 40$ & 2 & calcein & $10 \mathrm{mg} / \mathrm{L}$ \\
\hline e & $1 / 20 / 0818: 00$ & $1 / 21 / 088: 15$ & 2 & calcein & $10 \mathrm{mg} / \mathrm{L}$ \\
\hline e & $1 / 21 / 088: 30$ & $1 / 21 / 0817: 00$ & 2 & Alizarin & $5 \mathrm{mg} / \mathrm{L}$ \\
\hline $\mathrm{f}$ & $1 / 22 / 0818: 20$ & $1 / 23 / 088: 30$ & 2 & calcein & $10 \mathrm{mg} / \mathrm{L}$ \\
\hline f & $1 / 23 / 088: 50$ & $1 / 23 / 0818: 30$ & 2 & AC & $10 \mathrm{mg} / \mathrm{L}$ \\
\hline $\mathrm{g}$ & $1 / 23 / 0819: 40$ & $1 / 24 / 088: 00$ & $2^{*}$ & calcein & $10 \mathrm{mg} / \mathrm{L}$ \\
\hline $\mathrm{g}$ & $1 / 24 / 088: 35$ & $1 / 24 / 0817: 00$ & $2^{*}$ & Alizarin & $5 \mathrm{mg} / \mathrm{L}$ \\
\hline $\mathrm{g}$ & $1 / 24 / 0818: 30$ & $1 / 25 / 087: 50$ & $2^{*}$ & AC & $10 \mathrm{mg} / \mathrm{L}$ \\
\hline
\end{tabular}



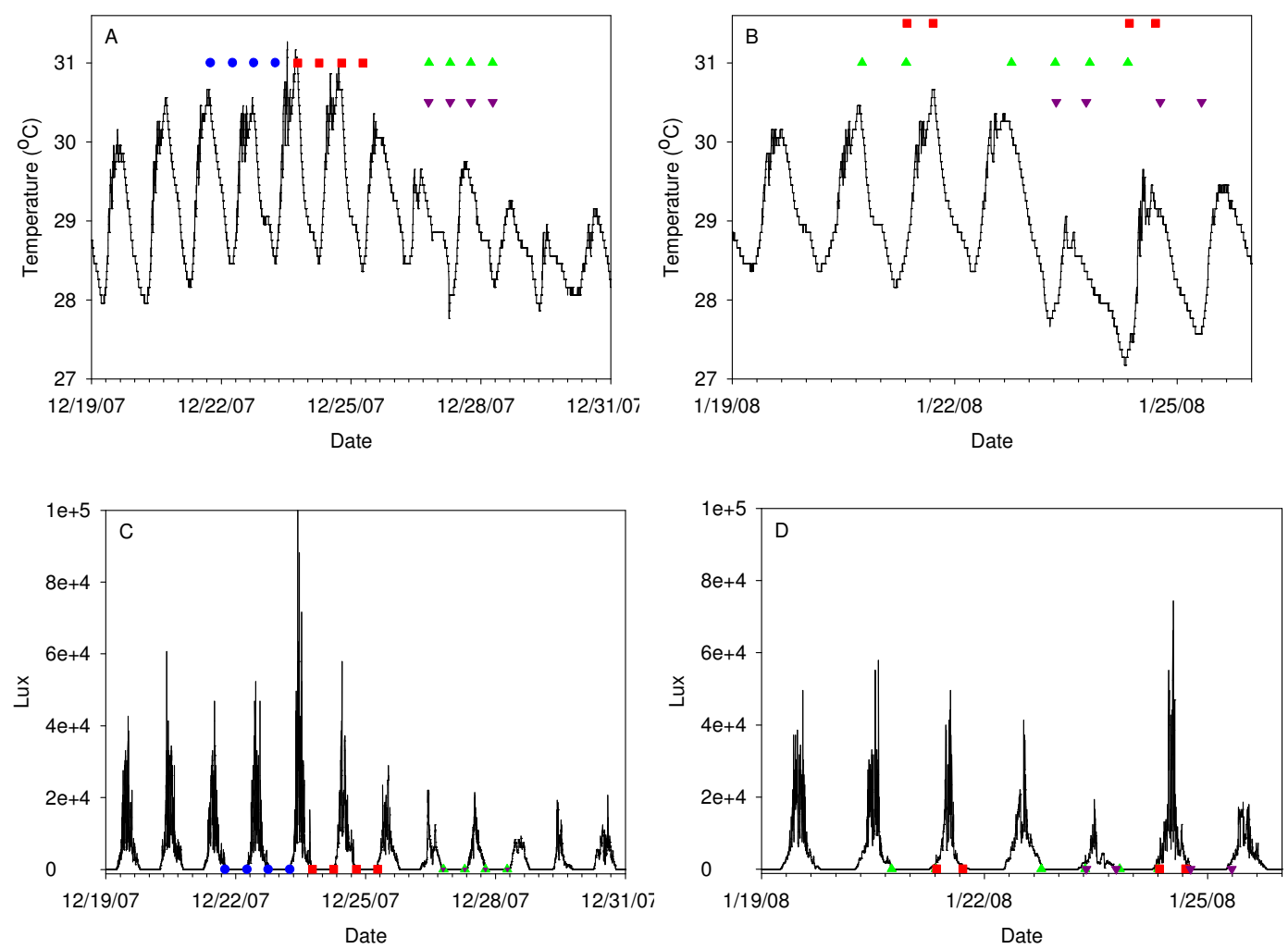

Figure 1. A, B Temperature $\left({ }^{\circ} \mathrm{C}\right)$ and C, D light intensity (lux) measured at the time of dye additions. Blue dots indicate the time of oxytetracycline addition and removal, red squares alizarin, and blue triangles calcein, and purple down triangles alizarin complexone. 

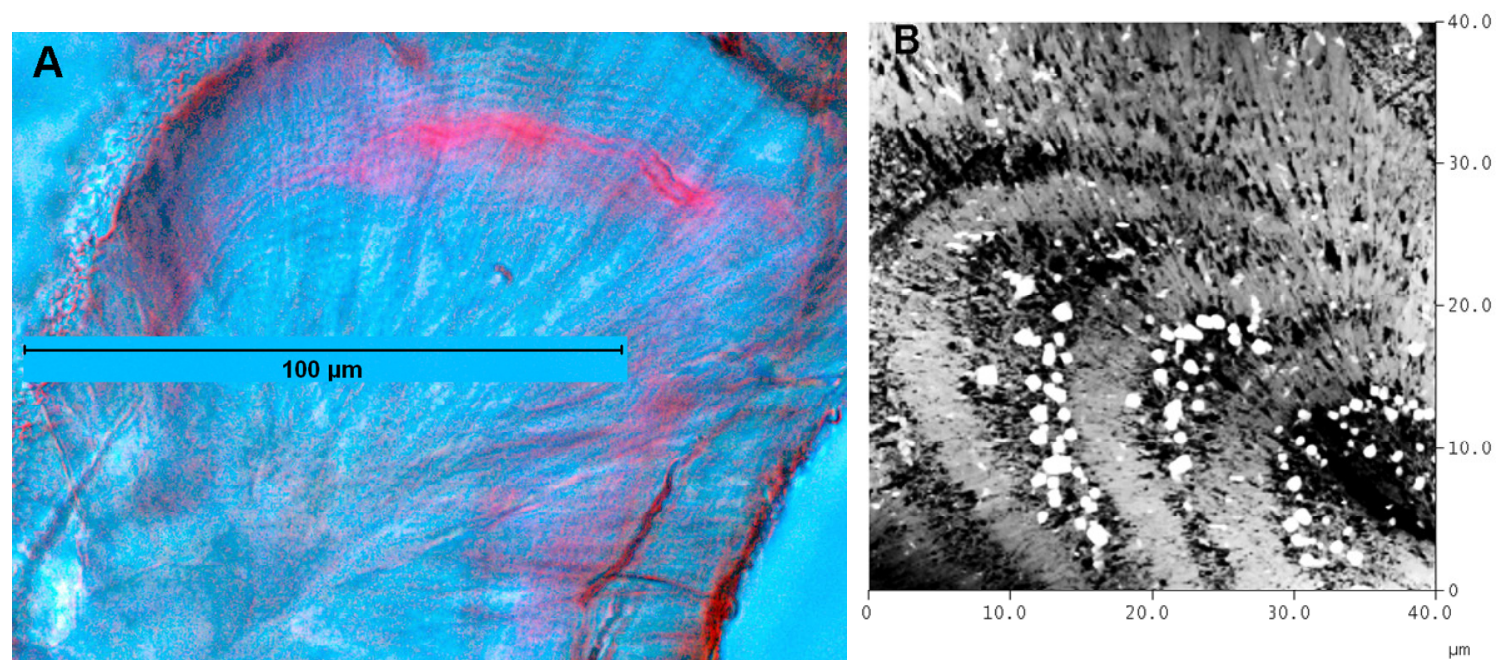

Figure 2. Banded coral skeleton sections imaged with polarized transmitted light microcopy (A), and with atomic force microscopy (B). In A, a banded region, indicated by the black arrow, is visible near the top of the image. Two nighttime alizarin stain lines (red bands) are visible in this section of Goniastrea edwardsi. Alizarin was applied at a concentration of 8 or $10 \mathrm{mg} / \mathrm{L}$ on two successive nights (18:30-6:15 and 18:50-6:40). In B, an AFM height image of a banded region in a section of a Diploria skeleton is shown. Higher regions (lighter) tend to correspond to aragonite fibers (F), and appear light in light microscopy. Lower regions (dark) correspond to fine granular crystals (blue arrow), and appear dark in light microscopy. 


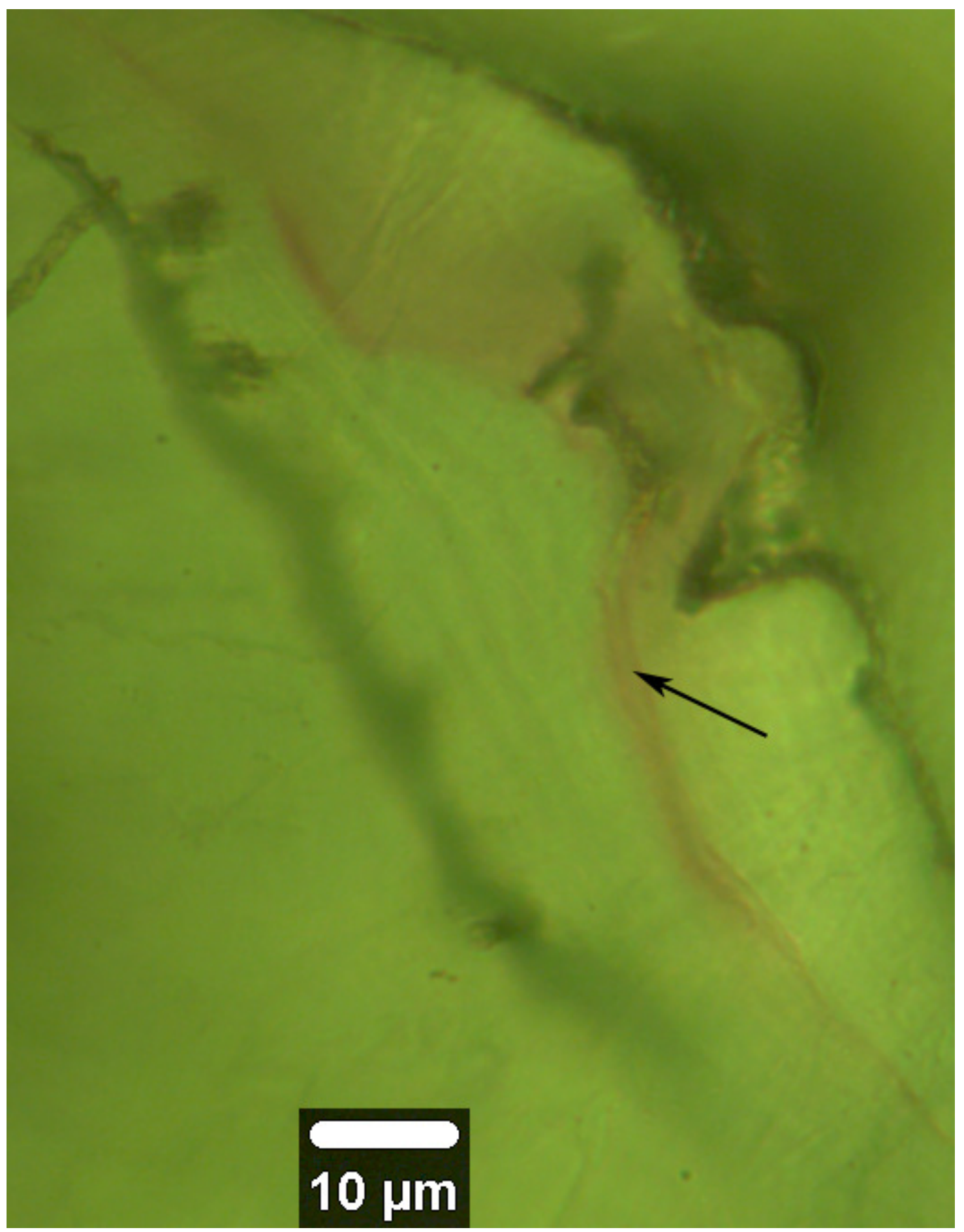

Figure 3. Transmitted light image of alizarin stained Porites lutea section. Alizarin red S, sodium salt $(8-10 \mathrm{mg} / \mathrm{L})$ was added on two successive nights (18:30-6:15 and 18:50-6:40) and appears to mark dark bands. A light band separating the two stain-lines is indicated by the arrow. 

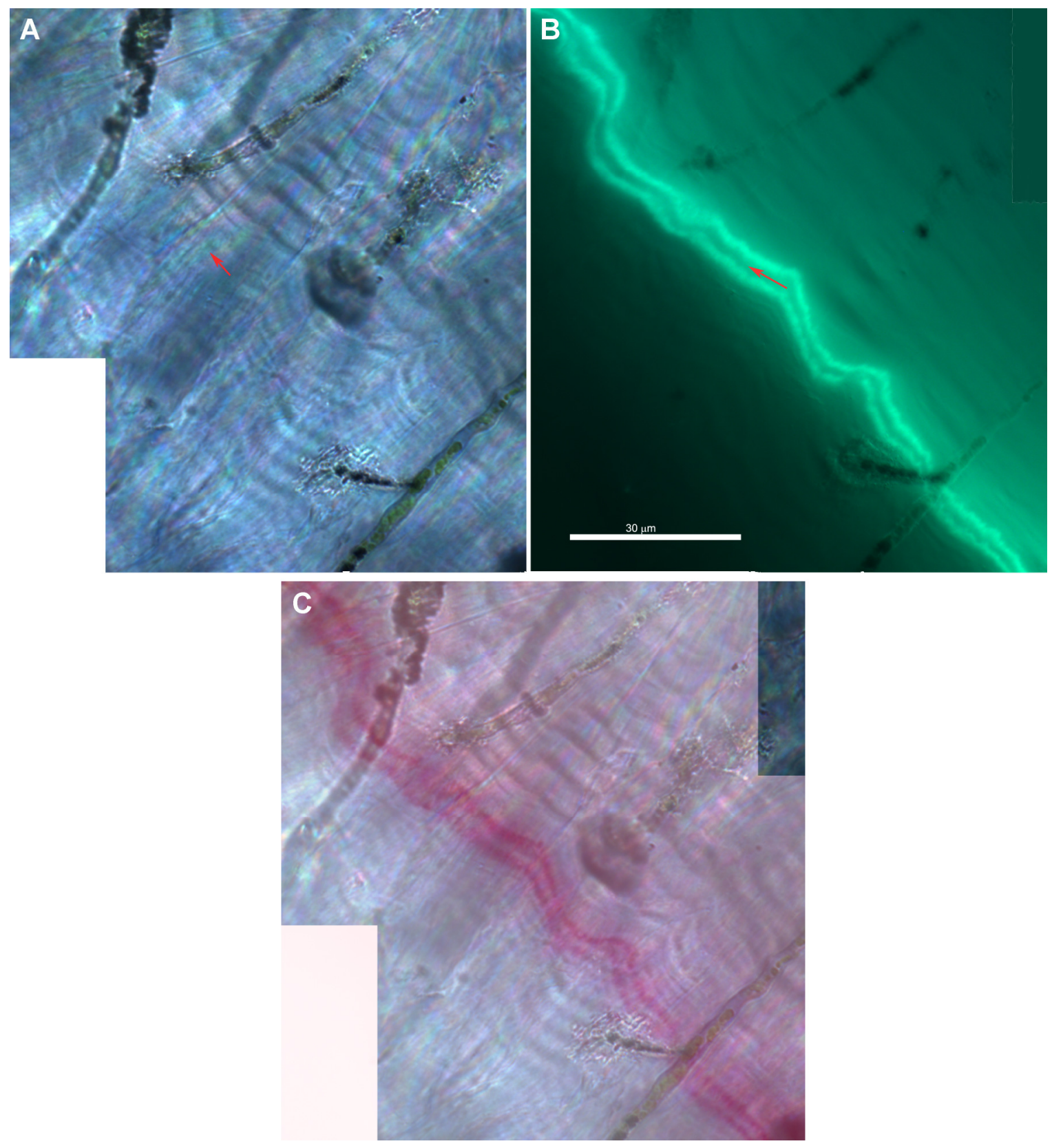

Figure 4. Calcein stained Goniastrea edwardsi section. Calcein $(10 \mathrm{mg} / \mathrm{L})$ was added on two successive nights (19:10-7:00 and 18:25-6:40) and appears to mark dark bands. A. Transmitted light image. B. Fluorescence image. C. Overlay image with the color scale inverted for the fluorescence image. In $\mathrm{A}$ and $\mathrm{B}$, the arrow points to a light band separating two stained dark bands. All images are on the same scale. 

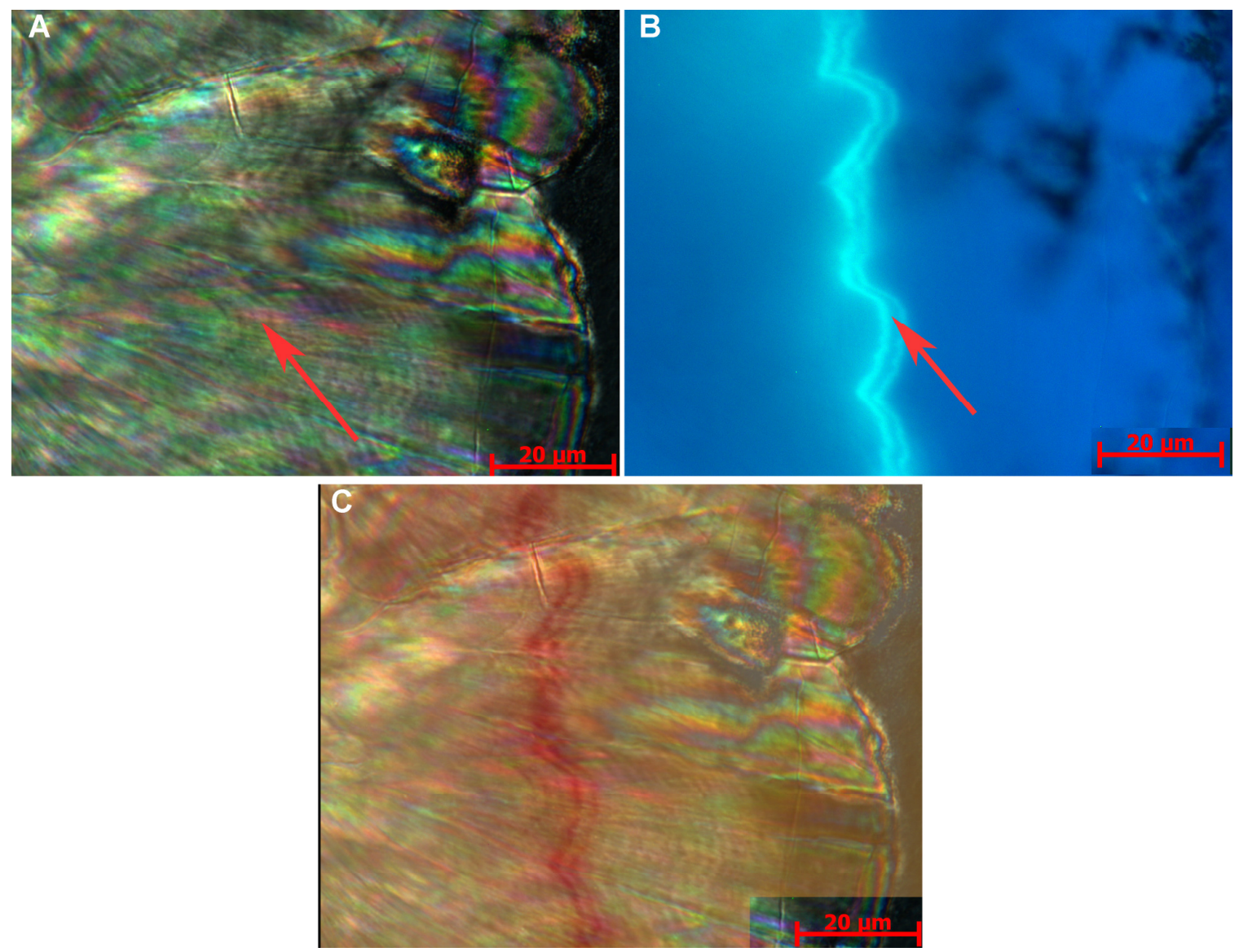

Figure 5. Oxytetracycline stained G. edwardsi section. Oxytetracycline (40 mg/L) was added on two successive nights (18:00-6:15 and 18:00-6:00) and appears to mark dark bands. A. Transmitted light image. B. Fluorescence image. C. Overlay image with the color scale inverted for the fluorescence image. In A and B, the arrow points to a light band separating two stained dark bands. 

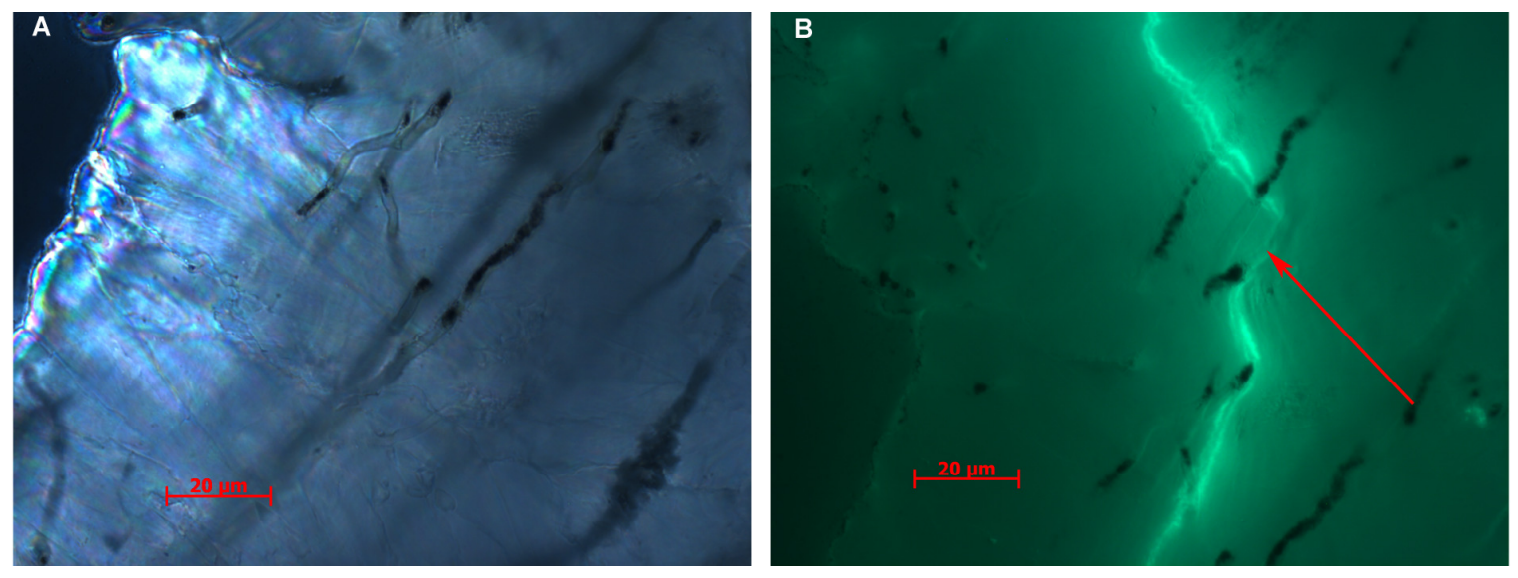

Figure 6. Calcein stained G. edwardsi section (same section as shown in Fig. 4). A. Transmitted light image. B. Fluorescence image. Images are not aligned due to too few homologous features being visible in both images. In B, the arrow points to a region where the stain line appears to disappear. 

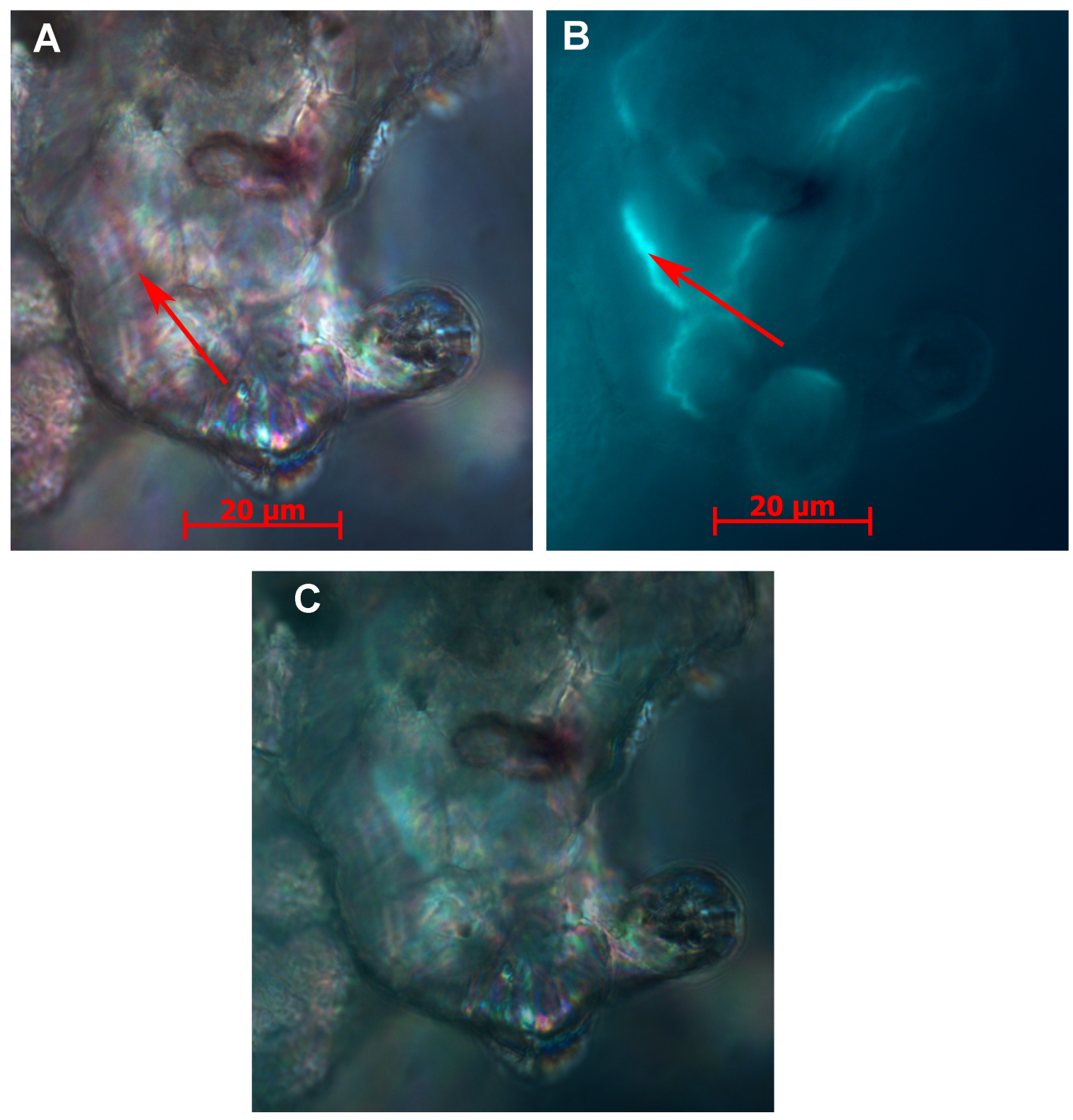

Figure 7. Calcein, alizarin, and alizarin complexone stained $P$. lutea section. Calcein (10 $\mathrm{mg} / \mathrm{L})$ was added on the first night (19:40-8:00), alizarin $(5 \mathrm{mg} / \mathrm{L})$ during the day $(8: 35-$ 17:00), and alizarin complexone $(10 \mathrm{mg} / \mathrm{L})$ on the following night (18:30-7:50). A.

Transmitted light image with alizarin staining corresponding to a light band indicated by an arrow. B. Fluorescence image with fluorescence indicated by an arrow. C. Overlay of image $\mathrm{B}$ on image $\mathrm{A}$ with the color scale inverted for the fluorescence image. Image alignment is less precise than for Figures 4 and 5, as only a single well defined feature was visible in both transmitted and fluorescence images that could be used for alignment. 

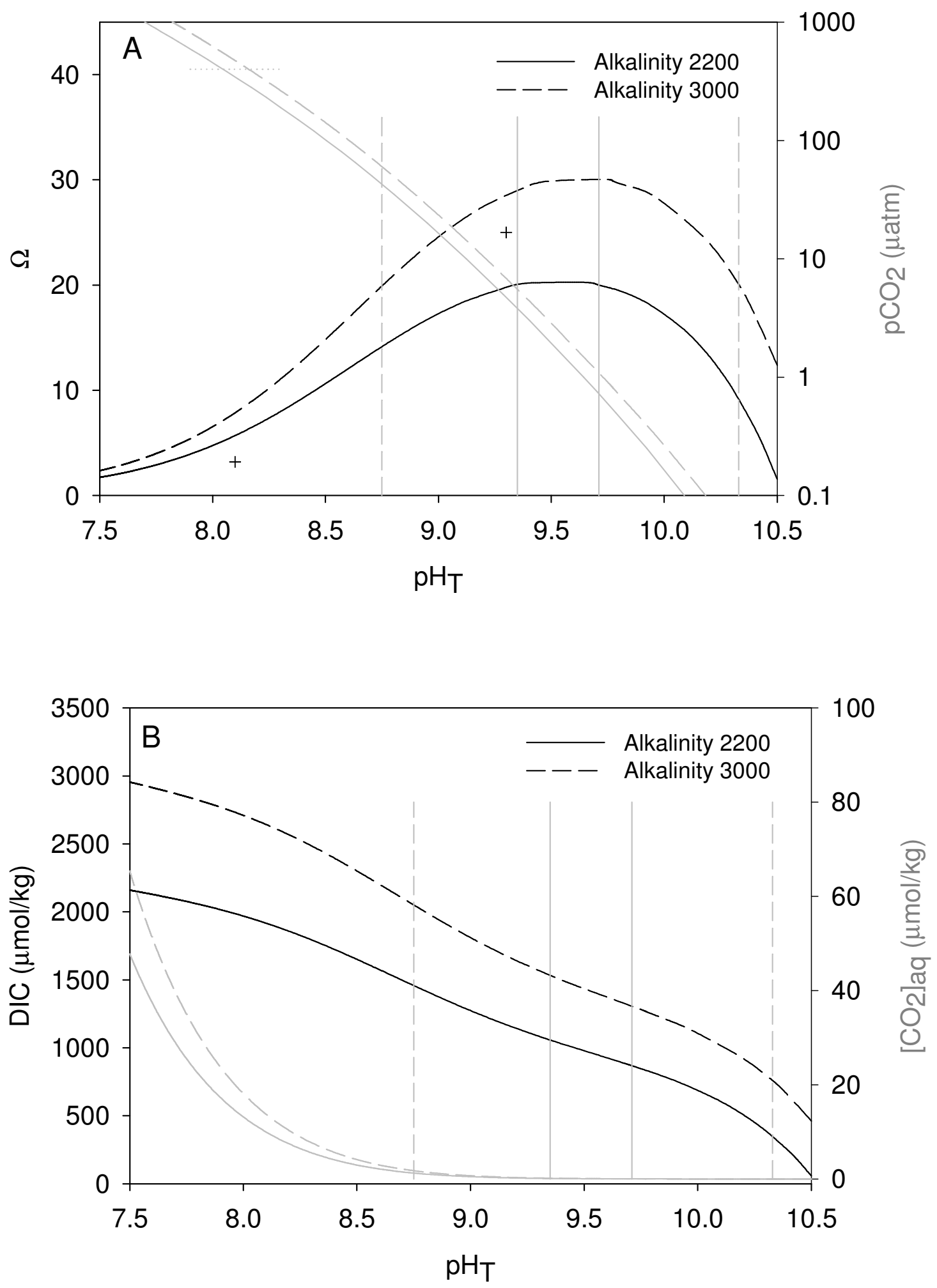
Figure 8. Effect of $\mathrm{CO}_{2}$ removal on the saturation state, $\mathrm{pCO}_{2}, \mathrm{DIC},\left[\mathrm{CO}_{2}\right]_{\mathrm{aq}}$, and $\mathrm{pH}$ of seawater held at constant alkalinity. The $\mathrm{x}$-axis is $\mathrm{pH}$, higher values correspond to more $\mathrm{CO}_{2}$ removal and thus lower $\mathrm{pCO}_{2}$. A. The calculated saturation state with respect to aragonite $(\Omega)$ (black lines plotted on left y-axis) and $\mathrm{pCO}_{2}$ (grey lines plotted on right yaxis - note this axis is on a log scale) is plotted versus $\mathrm{pH}$. For reference, the high (light) and low (dark) $\mathrm{pH}$ measurements and saturation state estimates from Al-Horani et al. (2003) are shown as black crosses. A dotted grey line in the upper left indicates 400 $\mu$ atm $\mathrm{CO}_{2}$. B. The DIC concentration (black lines plotted on left y-axis) and $\left[\mathrm{CO}_{2}\right]_{\mathrm{aq}}$ (grey lines plotted on right $\mathrm{y}$-axis) is plotted versus $\mathrm{pH}$. Values are plotted for two alkalinity levels, values corresponding to $2200 \mu \mathrm{mol} / \mathrm{kg}$ alkalinity are shown with solid lines, $3000 \mu \mathrm{mol} / \mathrm{kg}$ alkalinity with dashed lines. In each plot, a pair of vertical light grey lines connecting to the $\mathrm{x}$-axis represents the $\mathrm{pH}$ window in which granular crystal formation is likely to occur for a given alkalinity value. A Matlab implementation of CO2SYS which incorporates ammonia (constants from Clegg and Whitfield 1995) and allows other species and associated $\mathrm{pKa}$ values to be included was used for calculations. Input conditions were as follows $\mathrm{S}=30$, atmospheric pressure = $1 \mathrm{~atm}, \mathrm{Si}, \mathrm{PO}_{4}, \mathrm{NH}_{4}, \mathrm{HS}$, Tris, $\mathrm{AMP}=0, \mathrm{Ca}=15 \mathrm{mmol} / \mathrm{kg}$ (a higher than ambient value was used since corals concentrate Ca e.g., Marshall et al., (2007)), T=25. Constants of Mehrbach et al. (1973) as refit by Dickson and Millero (1987) were used for carbonate speciation, Dickson (1990) for sulfate. 

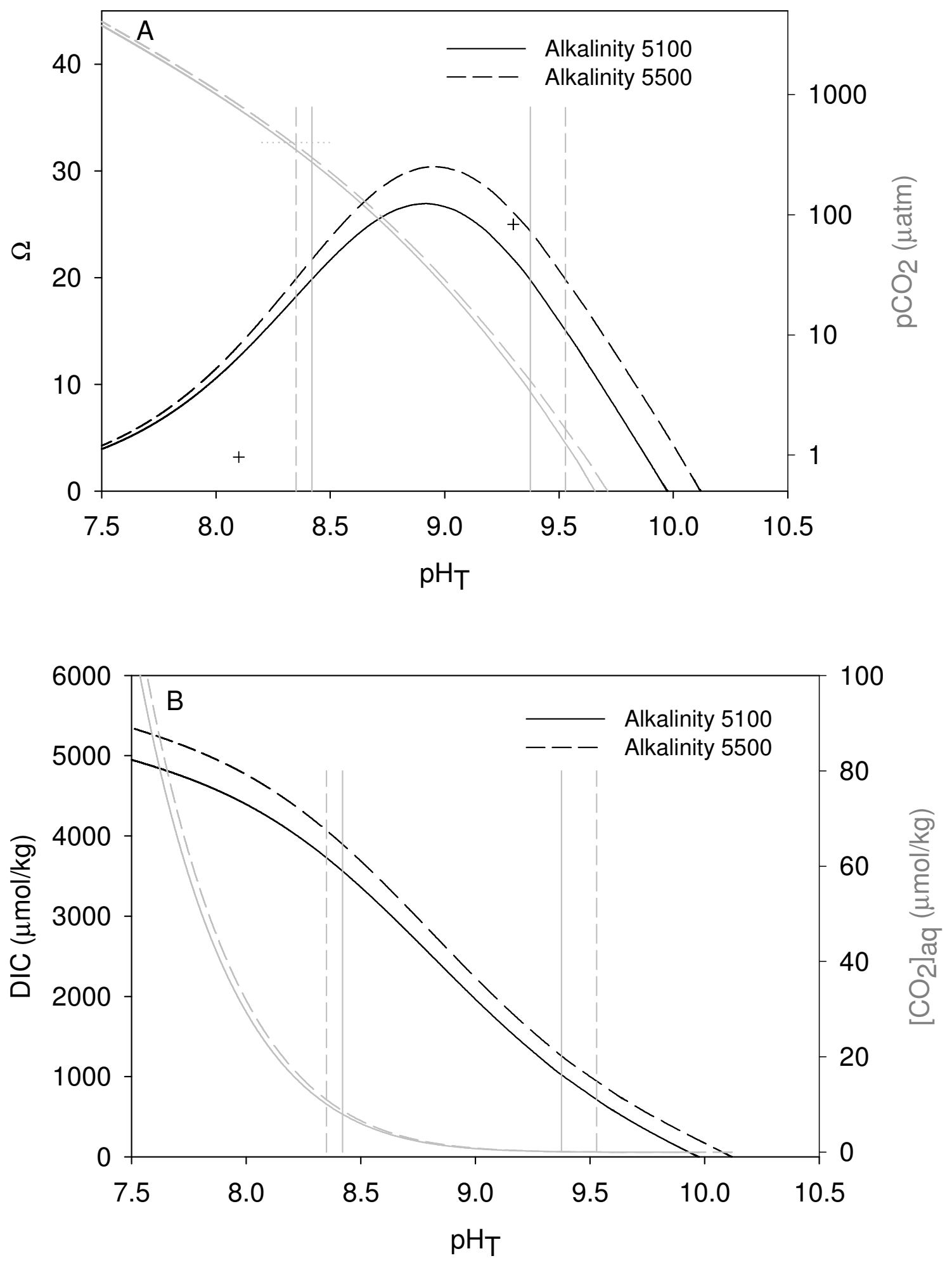
Figure 9. Effect of $\mathrm{CO}_{2}$ removal on the saturation state, $\mathrm{pCO}_{2}, \mathrm{DIC},\left[\mathrm{CO}_{2}\right]_{\mathrm{aq}}$, and $\mathrm{pH}$ of seawater with $5000 \mu \mathrm{mol} / \mathrm{kg} \mathrm{NH}_{3}$ held at constant alkalinity. The $\mathrm{x}$-axis is $\mathrm{pH}$, higher values correspond to more $\mathrm{CO}_{2}$ removal and thus lower $\mathrm{pCO}_{2}$. A. The calculated saturation state with respect to aragonite $(\Omega)$ (black lines plotted on left y-axis) and $\mathrm{pCO}_{2}$ (grey lines plotted on right y-axis - note this axis is on a log scale) is plotted versus $\mathrm{pH}$. For reference, the high (light) and low (dark) $\mathrm{pH}$ measurements and saturation state estimates from Al-Horani et al. (2003) are shown as black crosses. A dotted grey line in the upper left indicates $400 \mu \mathrm{atm} \mathrm{CO}_{2}$. B. The DIC concentration (black lines plotted on left $\mathrm{y}$-axis) and $\left[\mathrm{CO}_{2}\right]_{\mathrm{aq}}$ (grey lines plotted on right $\mathrm{y}$-axis) are plotted versus $\mathrm{pH}$. Values are plotted for two alkalinity levels, values corresponding to $5100 \mu \mathrm{mol} / \mathrm{kg}$ alkalinity are shown with solid lines, $5500 \mu \mathrm{mol} / \mathrm{kg}$ alkalinity with dashed lines. In each plot, a pair of vertical light grey lines connecting to the $\mathrm{x}$-axis represents the $\mathrm{pH}$ window in which granular crystal formation is likely to occur for a given alkalinity value. Conditions are per Fig. 8, except $5 \mathrm{mmol} / \mathrm{kg} \mathrm{NH} 3$ was included. 


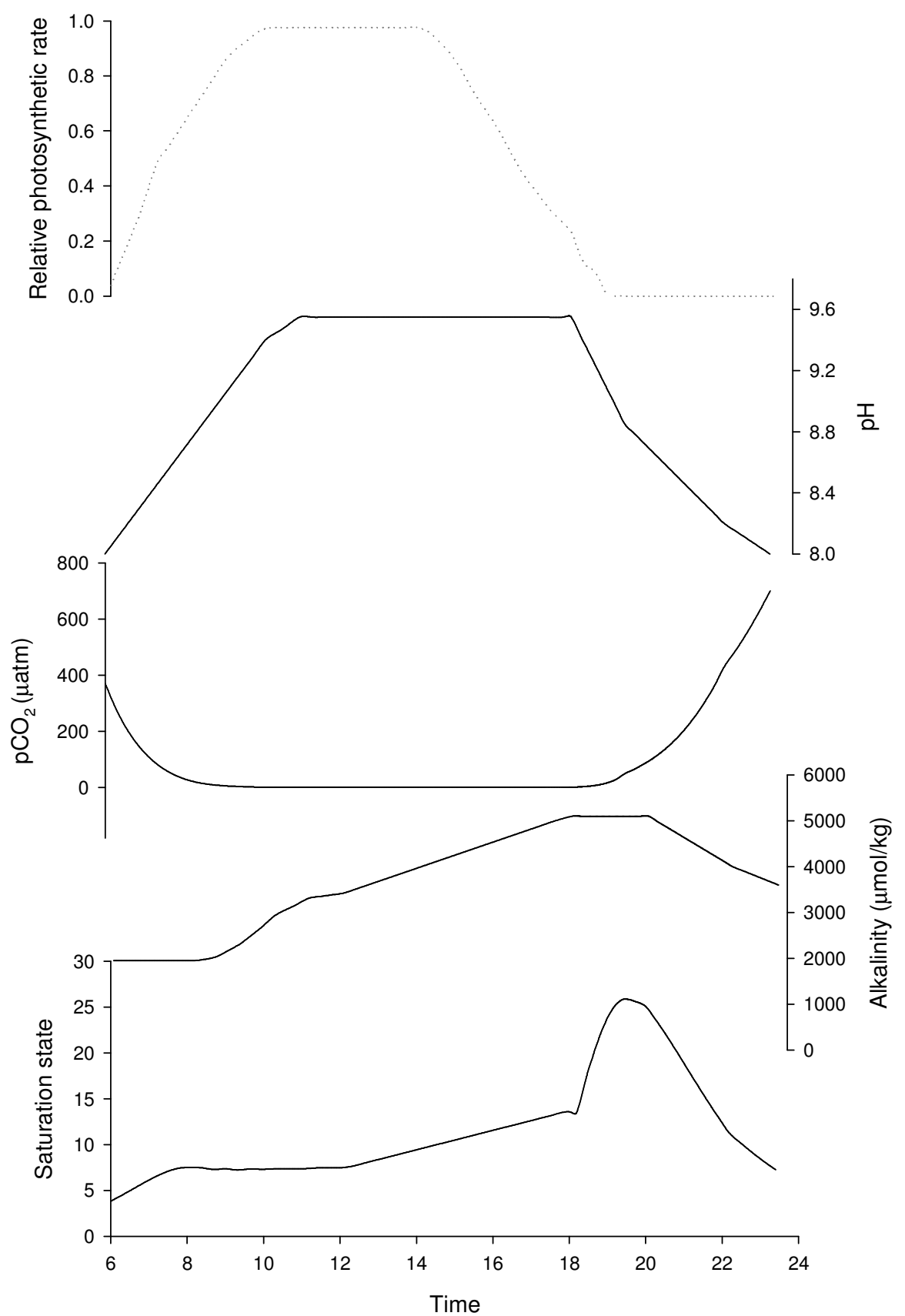

Figure 10. Idealized daily profiles of photosynthesis by the zooxanthellae and corresponding chemistry ( $\mathrm{pH}$, alkalinity, $\mathrm{pCO}_{2}$, and saturation state) at the site of calcification. Light levels and photosynthesis are assumed to follow $\sim$ natural daily cycles with levels being relatively low at sunrise, increasing throughout the morning to reach a saturating photosynthetic rate, and this saturated value is assumed to be maintained until light levels fall below saturation in the late afternoon. Alkalinity elevation is assumed to result primarily from accumulation of non-carbonate buffers. 
Chapter 3: Long-term effects of nutrient and $\mathrm{CO}_{2}$ enrichment on the temperate coral Astrangia poculata (Ellis and Solander, 1786)

\begin{abstract}
Zooxanthellate colonies of the scleractinian coral Astrangia poculata were grown under combinations of ambient and elevated nutrient $\left(5 \mu \mathrm{M} \mathrm{NO}_{3}{ }^{-}, 0.3 \mu \mathrm{M} \mathrm{PO}_{4}{ }^{-3}\right.$, and 2 $\left.\mathrm{nM} \mathrm{Fe}{ }^{+2}\right)$ and $\mathrm{CO}_{2}(\sim 780 \mathrm{ppmv})$ treatments for a period of 6 months. Coral calcification rates, estimated from buoyant weights, were not significantly affected by elevated nutrients at ambient $\mathrm{CO}_{2}$ and were negatively affected by elevated $\mathrm{CO}_{2}$ at ambient nutrient levels. However, calcification by corals reared under elevated nutrients combined with elevated $\mathrm{CO}_{2}$ was not significantly different from that of corals reared under ambient conditions, suggesting that $\mathrm{CO}_{2}$ enrichment can lead to nutrient limitation in zooxanthellate corals. A conceptual model is proposed to explain how nutrients and $\mathrm{CO}_{2}$ interact to control zooxanthellate coral calcification. Nutrient limited corals are unable to utilize an increase in dissolved inorganic carbon (DIC) as nutrients are already limiting growth, thus the effect of elevated $\mathrm{CO}_{2}$ on saturation state drives the calcification response. Under nutrient replete conditions, corals may have the ability to utilize more DIC, thus the calcification response to $\mathrm{CO}_{2}$ becomes the product of a negative effect on saturation state and a positive effect on gross carbon fixation, depending upon which dominates, the calcification response can be either positive or negative. This may help explain how the range of coral responses found in different studies of ocean acidification can be obtained.
\end{abstract}

Submitted to the Journal of Experimental Marine Biology and Ecology as: Long-term effects of nutrient and $\mathrm{CO}_{2}$ enrichment on the temperate coral Astrangia poculata (Ellis and Solander, 1786). M. Holcomb, D.C. McCorkle, A.L. Cohen 


\section{Introduction}

As the level of $\mathrm{CO}_{2}$ in the atmosphere and surface-ocean continues to rise, there is increasing concern about the impact of ocean acidification on corals and the diverse ecosystems they sustain. Many experimental studies have documented that scleractinian corals respond to ocean acidification and the consequent decline in aragonite saturation state $(\Omega)$ (e.g. Langdon and Atkinson, 2005), and most suggest that coral calcification decreases with decreasing saturation state. However, the saturation state of the surrounding seawater alone does not control coral calcification as corals can continue to calcify in under-saturated conditions (e.g. Marubini and Atkinson, 1999; Cohen et al., 2009) or dissolve in super-saturated conditions (e.g. Herfort et al., 2008).

In zooxanthellate corals, calcification appears tightly linked to photosynthesis by the symbiotic zooxanthellae. The median coral calcification rate in the light is three times that in the dark, and symbiotic corals calcify at higher rates than non-symbiotic corals (e.g. Gattuso et al., 1999). When corals bleach and lose their zooxanthellae, calcification rates decline (e.g. Rodrigues and Grottoli, 2006).

Photosynthesis generally responds positively to elevated $\mathrm{CO}_{2}$ and enhanced nutrient levels. Thus, high $\mathrm{CO}_{2}$ and high nutrient levels could enhance coral calcification. However, this is not necessarily the case. Elevated concentrations of inorganic nitrogen can increase zooxanthellae photosynthesis but cause calcification by the host coral to decline. Marubini and Davies (1996) suggested coral calcification and zooxanthellae photosynthesis compete for a limited supply of carbon. While nutrient enrichment favors growth of the zooxanthellae and increased photosynthetic activity, photosynthesis may 
out-compete calcification for a limited supply of dissolved inorganic carbon (DIC), so calcification declines.

Studies of ocean acidification show that coral calcification generally declines under higher $\mathrm{pCO}_{2}$ conditions, even in those studies where photosynthesis increases (e.g. Langdon and Atkinson, 2005). Thus, elevated $\mathrm{CO}_{2}$ levels and elevated nutrient levels each tend to negatively affect coral calcification. However, if the $\mathrm{CO}_{2}$ limitation model (Marubini and Davies, 1996) is correct, then the combined effects of elevated $\mathrm{CO}_{2}$ and nutrients could be positive. Nutrient addition may enhance photosynthesis while elevated DIC could relieve the competition between photosynthesis and calcification for DIC.

To date, four studies have specifically addressed the interaction of nutrients and carbonate chemistry on calcification (Marubini and Atkinson, 1999; Marubini and Thake, 1999; Langdon and Atkinson, 2005; Renegar and Riegl, 2005). Both the work of Marubini and Thake (1999) and of Marubini and Atkinson (1999) looked only at nitrogen, yet other nutrients, such as $\mathrm{P}$ and Fe have been found to be limiting in many systems (e.g. Sakka et al., 1999). In three of the four studies, carbonate chemistry was manipulated by the addition of acid/base and bicarbonate. However, the effects of $\mathrm{CO}_{2}$ may differ from those of acid addition (e.g. Jury et al., 2008). The one study to combine $\mathrm{CO}_{2}$ and nutrients incurred high rates of coral mortality (Renegar and Riegl, 2005), which makes interpretation of calcification rates difficult.

To assess how $\mathrm{CO}_{2}$ and nutrients $(\mathrm{N}, \mathrm{P}, \mathrm{Fe}$ ) interact to affect coral calcification, this study examines a temperate coral Astrangia poculata, which is shown to naturally experience a wide range in aragonite saturation state. Corals were grown under long- 
term conditions of nutrient enrichment and reduced carbonate ion concentration, with aqueous carbonate chemistry manipulated by $\mathrm{CO}_{2}$ enrichment.

\section{Methods}

\subsection{Corals}

Five zooxanthellate and six azooxanthellate A. poculata colonies were collected from the Woods Hole Oceanographic Institution (WHOI) pier near the peak summer time water temperature in early August $\left(23.5^{\circ} \mathrm{C}\right)$ at depths from 3-9 m. Specimens were returned to the lab in seawater, fragmented into four or eight pieces and trimmed of visibly bored skeleton. The fragments were attached to acrylic slides using cyanoacrylate adhesive. Once the corals were attached to the slide, the slides were suspended vertically using cable ties. Specimens were divided amongst treatments such that either one or two specimens from each parent colony were in every treatment. No mortality was observed during the course of the experiment. Corals were allowed to acclimate to aquarium conditions for 1 month, then $\mathrm{CO}_{2}$ and nutrient levels were gradually increased over a period of 10 days to reach treatment levels.

\subsection{Aquaria}

Coral fragments were maintained in a flow-through aquarium system. Two $450 \mathrm{~L}$ reservoirs were filled daily with Vineyard Sound seawater (UV sterilized, $20 \mu \mathrm{m}$ filtered); water from a given reservoir was continuously pumped to a header tank. From each header tank, water was fed into gas mixing chambers to equilibrate the water with a $\mathrm{CO}_{2}$ /air mixture, and from there into valve manifolds used to deliver water to individual 
aquaria. Individual aquaria consisted of 1 L PET food service containers (Solo) held in $25.8 \pm 0.1^{\circ} \mathrm{C}$ water baths (temperature controlled by an Omega $\mathrm{CN} 1504$ controller using aquarium heaters and thermistor temperature probes). Each container was covered with a tight fitting PET lid with 3 holes in the lid - one for water, one for air, and one for feeding. Incoming water passed through a loop of tubing inside the water bath, then dripped in from the top of the container. Outgoing water exited by a hole in the side which limited the total volume held to $\sim 800 \mathrm{ml}$. Flow rates to each container were $\sim 8$ L/day. Each container was continuously bubbled using aquarium air-stones. Lighting was provided by three T5-HO bulbs (2 10000K bulbs, 1 actinic), on a $12 \mathrm{hr} \mathrm{1/d} \mathrm{cycle;}$ light levels ranged from 40-115 $\mu \mathrm{mol}$ photons $/ \mathrm{m}^{2} / \mathrm{s}$ (Apogee quantum sensor), no systematic differences existed between light levels received by different treatments. Each container contained two corals, one zooxanthellate and one azooxanthellate. However, since the azooxanthellate corals acquired symbionts over the course of the experiment, the results from these colonies are not extensively discussed. Corals were fed daily. On alternating days, corals were fed either freshly hatched brine shrimp or frozen brine shrimp supplemented with Selcon (American Marine). Every few weeks, containers were cleaned of algal growth.

Based on previous studies in Vineyard Sound seawater (e.g. Glibert et al., 1985) dissolved inorganic nitrogen is expected to be variable, but always $<1 \mu \mathrm{M}$, and is most likely to be the limiting nutrient species. At $5 \mu \mathrm{M}$ the effect of $\mathrm{NO}_{3}{ }^{-}$on calcification has been found to saturate in tropical corals (Marubini and Davies 1996). Thus a target enrichment of $5 \mu \mathrm{M} \mathrm{NO}_{3}{ }^{-}$was chosen for the nutrient treatment to provide a 
concentration which should give the maximal calcification response and exceed the natural variability. As a precaution against inducing $\mathrm{PO}_{4}{ }^{3-}$ or $\mathrm{Fe}$ limitation, both were introduced in approximate Redfield ratios based on the $\mathrm{NO}_{3}{ }^{-}$enrichment. Nutrient levels were elevated by adding $\mathrm{NaNO}_{3}, \mathrm{~K}_{2} \mathrm{HPO}_{4}$, and $\mathrm{FeCl}_{2}$ stock solutions to one of the reservoirs to increase concentrations of $\mathrm{NO}_{3}{ }^{-}, \mathrm{PO}_{4}{ }^{-3}$, and $\mathrm{Fe}^{+2}$ by $5 \mu \mathrm{M}, 0.3 \mu \mathrm{M}$ and $2 \mathrm{nM}$ respectively. Water samples were taken every few days from each reservoir. Samples were stored frozen until analyzed for ammonium, nitrate/nitrite (expressed as $\mathrm{NO}_{3}$ ) and phosphate by the WHOI Nutrient Analytical Facility (using a Lachat Instruments QuickChem 8000 flow injection system). Mean levels were $3 \pm 4 \mu \mathrm{M} \mathrm{NH}_{4}, 6 \pm 1 \mu \mathrm{M} \mathrm{NO}_{3}$, $0.7 \pm 0.2 \mu \mathrm{M} \mathrm{PO}_{4}$ in the nutrient enriched treatment; ambient conditions were $3 \pm 5 \mu \mathrm{M}$ $\mathrm{NH}_{4}, 0.8 \pm 0.7 \mu \mathrm{M} \mathrm{NO}_{3}, 0.5 \pm 0.3 \mu \mathrm{M} \mathrm{PO}_{4}$, iron was not measured. The average difference between nutrient enriched and ambient samples collected on the same day were $0 \pm 6 \mu \mathrm{M}$ $\mathrm{NH}_{4}, 5 \pm 1 \mu \mathrm{M} \mathrm{NO}_{3}, 0.3 \pm 0.1 \mu \mathrm{MPO}_{4}$. Average nutrient levels experienced by the corals may have been lower due to algal growth between cleanings.

$\mathrm{CO}_{2}$ levels were increased for one set of gas mixing chambers and their corresponding individual aquaria using ambient air (provided by an air-compressor) and $\mathrm{CO}_{2}$. Flow rates for $\mathrm{CO}_{2}$ and air were controlled by rotameters (Alborg Instruments), to provide an air/ $\mathrm{CO}_{2}$ mixture with $\mathrm{CO}_{2}$ levels $\sim 400$ ppm above ambient, this value was chosen to provide an enrichment greater than daily variations in aquarium $\mathrm{pCO}_{2}$, while still being a value atmospheric $\mathrm{pCO}_{2}$ may reach within the next 100 years (e.g. Joos et al., 1999). A Qubit s151 $\mathrm{CO}_{2}$ analyzer and a commercially prepared $\mathrm{CO}_{2}$ standard (Corp Brothers) were used daily to assess the stability of $\mathrm{CO}_{2}$ levels. Average ambient $\mathrm{CO}_{2}$ 
levels were $386 \pm 9$ ppmv, $\mathrm{CO}_{2}$ enriched levels were $780 \pm 40$ ppmv. A dial barometer (Fisher) was used to measure atmospheric pressure at the time of each $\mathrm{CO}_{2}$ measurement (766 $\pm 7 \mathrm{mmHg})$. Alkalinity was measured as follows: Large volume alkalinity samples were taken periodically from the WHOI pier (in Great Harbor, Woods Hole, MA) at a depth of $1.5 \mathrm{~m}$ starting before the start of experiments and continuing after the completion of experiments to monitor ambient conditions. A single large volume sample was taken from the water supply used to fill the reservoirs feeding the coral tanks during the course of experiments (alkalinity: $2086 \mu \mathrm{mol} / \mathrm{kg}$, dissolved inorganic carbon (DIC): 1930 umol/kg, salinity: 32.02, sample taken Feb. 13, 2008). Samples were collected in $250 \mathrm{ml}$ glass bottles and poisoned with $70 \mu \mathrm{l}$ of a saturated $\mathrm{HgCl}_{2}$ solution. Alkalinity and DIC were determined by closed vessel titration of $\sim 100 \mathrm{ml}$ aliquots using an automated titration system (Bradshaw et al., 1981; Brewer et al., 1986). Alkalinity and DIC concentrations were determined using a nonlinear curve fitting approach (Department of Energy, 1994), and standardized using certified reference materials obtained from Dr. A. Dickson (Scripps Institution of Oceanography).

Samples for salinity measurements were taken at the same time as large volume alkalinity samples and were analyzed using a Guildline autosal model 8400B salinometer. Small volume alkalinity samples were taken four times over the course of the experiment from the reservoirs feeding the coral tanks. Small volume samples were stored at $4^{\circ} \mathrm{C}$ in screw cap vials (Evergreen Scientific) until analysis. Aliquots of $1 \mathrm{ml}$ were used for Gran titration with $0.01 \mathrm{~N} \mathrm{HCl}$; values ranged from $2050-2150 \mu \mathrm{mol} / \mathrm{kg}$, average $2110 \pm 40$ $\mu \mathrm{mol} / \mathrm{kg}$. 


\subsection{Buoyant weights}

Buoyant weight measurements (based on Davies 1989) were made one month after the start of treatments, and again five months later. An Acculab Vicon-123 balance (accurate to $0.01 \mathrm{~g}$ ) with weigh-below hook was used to weigh corals in ambient seawater. Glass and aluminum weights were used to estimate water density. Temperature and salinity were recorded at the same time. Corals were handled entirely underwater during buoyant weights to minimize stress or introduction of bubbles. Mass changes are assumed to reflect changes in the mass of aragonite; an aragonite density of 2.93 was used to calculate the dry weight of calcium carbonate deposited.

\subsection{Alkalinity depletion}

At the beginning and end of the experiment, water flow to all containers was stopped. A Ba isotope spike was added $\left({ }^{135} \mathrm{Ba}\right.$ or ${ }^{137} \mathrm{Ba}$, sufficient to double the ambient Ba concentration), and containers held without water flow for $\sim 2$ days. Small volume alkalinity samples were taken from every container at the end of the incubation to estimate calcification rates. Calcification was assumed to be the only process affecting alkalinity, with 2 moles alkalinity consumed for every mole of carbonate produced. Alkalinity samples were stored prior to measurement during which time some samples evaporated. Salinity was checked using a refractometer at the time of measurement, and samples which had evaporated during storage were excluded.

\subsection{Surface area}

Coral specimens were photographed using a Canon Digital Rebel XTi camera, images were saved in a raw file format. Photoshop CS3 was used to estimate the surface 
area of coral specimens. 2D surface areas and average diameters were determined for each specimen from images taken looking down on the coral; from side views, specimen height was determined. The total number of polyps was also counted. In addition, average calyx diameter and depth was obtained by sectioning some of the specimens. The data presented are normalized to an estimated 3D surface area using an average radius from the $2 \mathrm{D}$ surface area and colony height to calculate the surface area of a cone ( $\pi \mathrm{x}$ radius $\mathrm{x}$ height). To account for the additional area contributed from individual polyps, the polyps were assumed to add an additional conical area minus the area of the base (to avoid double counting) ( $\pi$ x calice radius $\mathrm{x}$ calice depth $-\pi \mathrm{x}$ (calice radius $\left.)^{2}\right)$. The average calice radius was $3.6 \pm .5 \mathrm{~mm}$, depth $4.5 \pm 1.3 \mathrm{~mm}$. Individual calices are closely approximated by a cone, while coral fragments displayed considerable variability, ranging from nearly planar to roughly cylindrical, with most specimens representing a form in between planar and cylindrical; a cone was chosen as an intermediate form which could be applied to all specimens.

\subsection{Statistics}

A factorial ANOVA model, including the parent colony as a blocking variable, was run on rank transformed data using SAS. To make pair-wise comparisons, each treatment combination was considered a unique treatment for use in a 1-way ANOVA, Dunnett's procedure was used to compare each treatment combination to ambient; comparisons with $\mathrm{p}<0.05$ are considered statistically significant. For each ANOVA model, residuals were plotted against predicted values, against measured values, and on 
normal probability plots to assess violations of ANOVA assumptions. Linear regressions were performed using Systat. All data are given as mean \pm standard deviation.

\section{Results}

Alkalinity, DIC, salinity and temperature measurements from the A. poculata collection site in Woods Hole, MA are reported in Figure 1 and Table 1. These data correspond to an annual cycle in seawater aragonite saturation state ranging from $\sim 1.5$ in the winter to $\sim 2.5$ in the summer.

Carbonate chemistry and saturation state of the seawater for each treatment, calculated from average alkalinity, salinity, phosphate and $\mathrm{pCO}_{2}$ values are shown in Table 2. The elevated $\mathrm{CO}_{2}$ treatments have higher bicarbonate concentrations (1840 $\mu \mathrm{mol} \mathrm{HCO}_{3} / \mathrm{kg}$ for $\mathrm{CO}_{2}$ treatment, $1660 \mu \mathrm{mol} \mathrm{HCO}_{3} / \mathrm{kg}$ for ambient) and lower saturation state $(1.8$ v 3.0$)$ than ambient or nutrient only treatments.

Coral calcification rates determined using alkalinity depletion are compared with calcification rates determined from buoyant weight measurements in Figure 2. Data obtained by the two methods are significantly correlated $(\mathrm{p}<0.001)$, with a relationship described by the equation: buoyant weight value $=1.08 *$ alkalinity depletion value. Alkalinity depletion measurements were made for each container, thus the values represent the combined calcification by the zooxanthellate and the originally azooxanthellate colony in each container. The alkalinity depletion value presented is the average of measurements made at the beginning and end of each treatment. To enable comparison of the alkalinity depletion and buoyant weight methods of estimating 
calcification, the buoyant weights of the zooxanthallate and originally azooxanthellate colonies have been combined. Since both alkalinity depletion and buoyant weight based calcification estimates show similar patterns, it is possible to compare results of different studies in which only one of the two methods has been employed.

Figure 3 shows the average calcification rates for zooxanthellate $A$. poculata colonies grown for six months under each of the nutrient and $\mathrm{CO}_{2}$ treatments normalized

to surface area. Average growth rates were $2.1 \pm 0.1,0.7 \pm 0.1,1.4 \pm 0.1,1.3 \pm 0.1 \mathrm{~g} / \mathrm{m}^{2} / \mathrm{d}$ for ambient, $\mathrm{CO}_{2}$ treated, nutrient treated, and $\mathrm{CO}_{2}+$ nutrient treated corals respectively. Relative to ambient conditions, growth rates were reduced significantly by $\mathrm{CO}_{2}$ treatment $(p<0.05)$ to $34 \%$ of the ambient rate, however with the addition of nutrients, growth rates were $61 \%-65 \%$ of ambient regardless of $\mathrm{CO}_{2}$ level. Table 3 shows the associated factorial ANOVA table. Treatment with $\mathrm{CO}_{2}$ resulted in lower calcification rates $(\mathrm{p}=$ 0.0212). Nutrients alone appear to have a slightly negative effect, though this is not significant $(\mathrm{p}=0.7273)$. However, when combined with $\mathrm{CO}_{2}$, nutrients appear to reduce the negative effects of $\mathrm{CO}_{2}$, suggesting a potential interaction of nutrients with $\mathrm{CO}_{2}$, though the interaction term is not significant $(\mathrm{p}=0.0562)$.

\section{Discussion}

Elevated $\mathrm{pCO}_{2}$ levels negatively impacted calcification by A. poculata. However, the degree to which elevated $\mathrm{pCO}_{2}$ impacted calcification was affected by inorganic nutrients. The decline in calcification with elevated $\mathrm{pCO}_{2}$ at ambient nutrient levels most likely indicates a calcification response to changing carbonate chemistry (declining 
saturation state). Nutrient addition alone resulted in an apparent reduction in calcification of $35 \%$, consistent with suggestions of carbon limitation under nutrient enriched conditions (e.g. Marubini and Davies, 1996). The combination of nutrient addition and elevated $\mathrm{pCO}_{2}$, however, counteracted the negative effects of elevated $\mathrm{pCO}_{2}$ alone. This response is similar to that reported by Langdon and Atkinson (2005) for tropical corals (Figure 4). Despite using different methodologies ( $\mathrm{HCl}$ addition, constant DIC vs $\mathrm{pCO}_{2}$ elevation, elevated DIC) and coral species (temperate vs tropical), both studies show that elevated nutrient conditions reduce the degree to which reduced saturation state impacts coral calcification. This suggests that coral calcification can be nutrient limited under elevated DIC conditions (or elevated $\mathrm{HCO}_{3}{ }^{-}+\mathrm{CO}_{2}(\mathrm{aq}$ ), the two carbon species generally used for photosynthesis), while elevated nutrients can lead to DIC limitation. Thus, the response of $A$. poculata to changing $\mathrm{pCO}_{2}$ levels depends on the nutritional status of the coral holobiont, and the response to nutrients is similarly dependent upon DIC. Conversely, Renegar and Riegl (2005) found no benefit from adding nitrate or phosphate under elevated $\mathrm{CO}_{2}$ conditions, however, in their study, DIC was lower in the high $\mathrm{CO}_{2}$ treatment. Similarly, Marubini and Atkinson (1999) found no benefit from adding nutrients under acidified conditions, however, their acidification treatment was also associated with lower DIC.

Based on the results reported here, and by Langdon and Atkinson (2005), it may be possible to make a general prediction as to how otherwise healthy corals could respond to elevated $\mathrm{pCO}_{2}$ depending on the availability of nutrients or their nutritional status. Nutritionally replete corals should be able to compensate for reduced saturation 
state under elevated $\mathrm{pCO}_{2}$ conditions. As $\mathrm{pCO} 2$ increases and seawater saturation state declines, availability of DIC to the zooxanthellae will increase, potentially allowing increased photosynthesis which provides both alkalinity and energy to help drive calcification. If the coral is experiencing carbon limitation, elevated $\mathrm{pCO}_{2}$ could even positively impact calcification. Thus, nutritionally replete corals are expected to be relatively insensitive to modest reductions in saturation state, and can potentially benefit from higher DIC levels. Conversely, elevation of nutrient levels may negatively impact calcification by nutritionally replete corals by increasing zooxanthellae growth which could increase photosynthesis leading to DIC limitation of calcification, or increase self shading of the zooxanthellae leading to light limitation and reduced photosynthetic efficiency (see Marubini and Davies 1996). Calcification by nutritionally limited corals will be strongly affected by reduced saturation state since DIC is already available at sufficient levels to support photosynthesis and calcification, thus the decline in saturation state should drive the calcification response. Nutritionally limited corals may thus respond in a manner more similar to the way inorganic aragonite precipitation responds to changing seawater saturation state (e.g., Burton and Walter, 1987). Nutritionally limited corals could benefit from modest nutrient increases so long factors such as light availability, available space, and a lack of competition with other organisms (e.g. Lapointe, 1997) can allow an increase in nutrients to translate into increased photosynthesis and increased transfer of photosynthate to the coral host, while still having sufficient DIC to support calcification. 
The interaction between nutritional status of the coral, DIC availability, and saturation state may help to explain the wide range of calcification responses seen in published acidification and nutrient enrichment studies. Figures 5 and 6 summarize data from several studies using a range of methods to manipulate carbonate chemistry and estimate calcification rates of both corals and coral communities. Only studies which provided sufficient data to calculate carbonate speciation are included. The change in coral calcification rates is presented as a function of saturation state, carbonate species, and nutrient levels. Across these studies, calcification rates were determined using one of four methods: alkalinity depletion and buoyant weight techniques, shown in this study to be comparable (Fig. 2), and radioisotope and calcium uptake techniques, also shown to be comparable to alkalinity depletion values by Tambutte et al. (1995) and Chisholm and Gattuso (1991).

In Figure 5 all but two of the studies show a positive relationship between saturation state and relative calcification rate. In inorganic precipitation studies, saturation state is closely tied to precipitation rate (e.g. Burton and Walter, 1987; Holcomb et al., 2009), thus it might be expected that precipitation of aragonite by corals would show a similar dependence on saturation state, and some studies do show a similar relationship (e.g. Langdon and Atkinson, 2005; Herfort et al., 2008; the current study) (Fig. 4, 5a). However, amongst those studies showing a positive response of calcification to calcification responds positively to saturation state, the calcification response differs by orders of magnitude. Such high levels of variability suggest that saturation state alone is not an effective predicator of coral calcification. Calcification rate responses to 
carbonate ion follow a similar pattern to the response to saturation state (Fig. 6a). For bicarbonate (Fig. 6b), aqueous $\mathrm{CO}_{2}$ (Fig. 6c), and DIC (Fig. 6d) some studies show positive relationships between calcification and bicarbonate, aqueous $\mathrm{CO}_{2}$, or DIC, while others show negative relationships. Such a wide range of responses suggests that no carbonate system parameter alone can be used as an effective predictor of coral calcification.

In studies which have examined the effects of feeding, nutrients, light, and temperature without manipulating carbonate chemistry, calcification rates changed substantially in the absence of substantial changes in seawater saturation state (e.g. Marubini and Davies, 1996; Reynaud et al., 2003; Houlbreque et al., 2004; Tanaka et al., 2007). Such results point to the importance of multiple factors in determining rates of coral calcification.

It has been suggested that bicarbonate may be the carbonate species controlling calcification rates (Herfort et al., 2008; Jury et al., 2008). However, Figure 6b, 6c, and 6d show no clear correlation between bicarbonate, aqueous $\mathrm{CO}_{2}$, or DIC and calcification. Most of the studies showing positive calcification rate responses to elevated bicarbonate, aqueous $\mathrm{CO}_{2}$, or DIC are studies in which saturation state is positively correlated with bicarbonate, aqueous $\mathrm{CO}_{2}$, or DIC. Thus saturation state may be the controlling factor. The work of Reynaud et al. (2003) done at $25^{\circ} \mathrm{C}$ and Anthony et al (2008) done at $\sim 28.5^{\circ} \mathrm{C}$ are exceptions, in which saturation state and calcification are not correlated. Anthony et al. (2008) used three $\mathrm{CO}_{2}$ levels, only the highest of which was associated with a decline in calcification. Both of these studies manipulated carbonate chemistry by 
use of $\mathrm{CO}_{2}$, and DIC increases as saturation state declines. These studies may represent nutrient replete conditions in which DIC is limiting calcification, thus a moderate elevation of DIC allows calcification to increase despite the decline in saturation state.

This study shows that elevated $\mathrm{pCO}_{2}$ negatively impacts calcification, but that the addition of nutrients $(\mathrm{N}, \mathrm{P}, \mathrm{Fe})$ can greatly reduce that impact. The interactions amongst 'nutrients', DIC, and saturation state yield results different from those seen when any one variable is manipulated. Aquarium-based experiments can therefore produce a wide range of coral responses to changes in seawater saturation state, all or none of which may be applicable to reef communities. In the studies discussed, only a small handful of the variables known to affect calcification and/or photosynthesis have been tested in combination with other variables, but how representative the specific values used may be of natural reef environments is unknown. Additional complications arise in aquarium systems with fluid flow which may be much lower than encountered in a reef environment, creating diffusive boundary layers that could artificially limit transport of ions to the coral, or removal of metabolic wastes thus altering the apparent sensitivity of corals to nutrient, DIC and saturation state changes. Aquarium experiments also fail to capture how reefs, which contain countless species, will respond to changes in nutrients and $\mathrm{CO}_{2}$ due to shifts in species abundance - macroalgae in particular are one group of organisms commonly artificially controlled in aquarium environments which may well benefit from higher nutrient levels and lead to a shift in community composition. Further laboratory-based work is needed to better understand how nutrients and/or enhanced nutritional status impact the calcification response to ocean acidification, and to identify 
measurable parameters, such as tissue composition, $\mathrm{C}: \mathrm{N}: \mathrm{P}$ ratios, etc. that may predict the 'nutritional' status of corals in natural settings. Further, by manipulating carbonate chemistry in natural reef environments, the coral calcification response to elevated $\mathrm{CO}_{2}$ could be more accurately quantified. 


\section{Acknowledgements}

The authors are grateful for the assistance of Dave Wellwood who analyzed salinity, Rebecca Belastock who performed alkalinity and DIC measurements, Joanne Goudreau who performed alkalinity measurements, Paul Henderson who analyzed nutrients, Scott Gallager and Ann Tarrant who provided helpful suggestions on running the experiment, Jason Smith who helped calibrate temperature probes, Amber York, Fred Keller and Kevin Thompson who help to keep the seawater supply running, Laura Hmelo, Christine Mingione, Byron Pedler, Casey Saenger, Kristen Whalen, and Terry Rioux for diving assistance, Alyson Bodendorf, Hana Keys, and Ellen Roosen for aquarium maintenance. Funding for this work was provided by the Ocean Life Institute, NSF OCE-0648157, and an International Society for Reef Studies / Ocean Conservancy Fellowship. This material is based upon work supported under a National Science Foundation Graduate Research Fellowship. 


\section{References}

Anthony, K.R.N., Kline, D.I., Diaz-Pulido, G., Dove, S., Hoegh-Guldberg, O., 2008. Ocean acidification causes bleaching and productivity loss in coral reef builders. Proc Natl Acad Sci USA 105, 17442-17446.

Bradshaw, A.L., Brewer, P.G., Shafer, D.K., Williams, R.T., 1981. Measurements of total carbon dioxide and alkalinity by potentiometric titration in the GEOSECS program. Earth Planet Sci Lett 55, 99-115.

Brewer, P.G., Bradshaw, A.L. Williams, R.T. 1986. Measurements of total carbon dioxide and alkalinity in the North Atlantic Ocean in 1981. In: Reichle, D. (Ed.), The Global Carbon Cycle: Analysis of the Natural Cycle and Implications of Anthropogenic Alterations for the Next Century, Springer-Verlag, New York, pp. 358-381.

Burton, E.A., Walter, L.M., 1987. Relative precipitation rates of aragonite and Mg calcite from seawater: Temperature or carbonate ion control? Geology 15, 111-114.

Chisholm, J.R.M., Gattuso, J.-P., 1991. Validation of the alkalinity anomaly technique for investigating calcification and photosynthesis in coral reef communities.

Limnol. Oceanography 36, 1232-1239.

Cohen, A.L., McConnaughey, T.A., 2003. Geochemical perspectives on coral mineralization. Reviews in Mineralogy Geochem: Biomineralization 54, 151187.

Davies PS (1989) Short-term growth measurements of corals using an accurate buoyant weighing technique. Marine Biol. 101:389-395

Dickson, A.G. 1990. Standard potential of the reaction: $\mathrm{AgCl}(\mathrm{s})+1 / 2 \mathrm{H} 2(\mathrm{~g})=\mathrm{Ag}(\mathrm{s})+$ $\mathrm{HCl}(\mathrm{aq})$, and the standard acidity constant of the ion HSO4- in synthetic seawater from 273.15 to $318.15 \mathrm{~K}$. J. Chem. Thermodynamics 22:113-127.

Dickson, A. G., and F. J. Millero. 1987. A comparison of the equilibrium constants for the dissociation of carbonic acid in seawater media. Deep-Sea Research 34:17331743.

DOE, eds, Dickson, A.G., Goyet, C., 1994. Handbook of Methods for the Analysis of the Various Parameters of the Carbon Dioxide System in Sea Water version 2. ORNL/CDIAC-74.

Gattuso, J.P., Allemand, D., Frankignoulle, M., 1999. Photosynthesis and calcification at cellular, organismal and community levels in coral reefs: a review of interactions and control by carbonate chemistry. Am Zool 39, 160-183.

Glibert PM, Dennett MR, Goldman JC (1985) Inorganic carbon uptake by phytoplankton in vineyard sound, massachusetts. I. Measurements of the photosynthesisirradiance response of winter and early-spring assemblages. J. Exp. Mar. Biol. Ecol. 85:21-36

Goreau, T.F., 1961. On the relation of calcification to primary productivity in reef building organisms. In: Lenhoff, H.M., Loomis, W.H. (Ed), The biology of hydra and some other coelenterates pp. 269-285.

Herfort, L., Thake, B., Taubner, I., 2008. Bicarbonate stimulation of calcification and photosynthesis in two hermatypic corals. J Phycol 44, 91-98. 
Holcomb, M., Cohen, A.L., Gabitov, R.I., Hutter, J.L., 2009. Compositional and morphological features of aragonite precipitated experimentally from seawater and biogenically by corals. Geochim Cosmochim Acta 73, 4166-4179.

Houlbreque, F., Tambutte, E., Allemand, D., Ferrier-Pages, C., 2004. Interactions between zooplankton feeding, photosynthesis and skeletal growth in the scleractinian coral Stylophora pistillata. J. Exp. Biol. 207, 1461-1469.

Joos F, Plattner GK, Stocker TF, Marchal O, Schmittner A (1999) Global warming and marine carbon cycle feedbacks on future atmospheric CO2. Science 284: 464-467

Jury, C., Whitehead, R., Szmant, A., 2008. Aragonite saturation state and seawater pH do not predict calcification rates of the reef-building coral Madracis mirabilis. 11th ICRS Fort Lauderdale, FL

Langdon, C., Atkinson, M.J., 2005. Effect of elevated pCO2 on photosynthesis and calcification of corals and interactions with seasonal change in temperature/irradiance and nutrient enrichment. J. Geophys. Res. 110.

Langdon, C., Takahashi, T., Sweeney, C., Chipman, D., Goddard, J., Marubini, F., Aceves, H., Barnett, H., Atkinson, M.J., 2000. Effect of calcium carbonate saturation state on the calcification rate of an experimental coral reef. Global Biogeochem Cycles 14, 639-654.

Langdon, C., Wallace S. Broecker, Douglas E. Hammond, Edward Glenn, Kevin Fitzsimmons, Steven G. Nelson, Tsung-Hung Peng, Irka Hajdas, Bonani, G., 2003. Effect of elevated CO2 on the community metabolism of an experimental coral reef. Global Biogeochem Cycles 17, 1011.

Lapointe, B. E. (1997), Nutrient threshold for bottom-up control of macroalgal blooms on coral reefs in Jamaica and Southeast Florida, Limnol. Oceanography 42, 1119- 1131.

Leclercq, N., Gattuso, J.P., Jaubert, J., 2000. CO2 partial pressure controls the calcification rate of a coral community. Global Change Biol. 6, 329-334.

Leclercq, N., Gattuso, J.-P., Jaubert, J., 2002. Primary production, respiration, and calcification of a coral reef mesocosm under increased $\mathrm{CO} 2$ partial pressure. Limnol. Oceanography 47, 558-564.

Marubini, F., Davies, P.S., 1996. Nitrate increases zooxanthellae population density and reduces skeletogenesis in corals. Marine Biol. 127, 319-328.

Marubini, F., Thake, B., 1999. Bicarbonate addition promotes coral growth. Limnol. Oceanography 44, 716-720.

Marubini, F., Atkinson, M.J., 1999. Effects of lowered pH and elevated nitrate on coral calcification. Mar. Ecol. Prog. Ser. 188, 117-121.

Marubini, F., Ferrier-Pages, C., Cuif, J.-P., 2003. Suppression of skeletal growth in scleractinian corals by decreasing ambient carbonate-ion concentration: a crossfamily comparison. Proc. R. Soc. Lond. B 270, 179-184.

Marubini, F., Barnett, H., Langdon, C., Atkinson, M.J., 2001. Dependence of calcification on light and carbonate ion concentration for the hermatypic coral Porites compressa. Mar. Ecol. Prog. Ser. 220, 153-162. 
Marubini, F., Ferrier-Pages, C., Furla, P., Allemand, D., 2008. Coral calcification responds to seawater acidification: a working hypothesis towards a physiological mechanism. Coral Reefs 27, 491-499.

Mehrbach, C., Culberson, C.H., Hawley, J.E., Pytkowicz, R.M., 1973. Measurement of the apparent dissociation constants of carbonic acid in seawater at atmospheric pressure. Limnol. Oceanography 18, 897-907.

Ohde, S., Hossain, M.M.M., 2004. Effect of CaCO3 (aragonite) saturation state of seawater on calcification of Porites coral. Geochem J 38, 613-621.

Pierrot, D. E. Lewis, and D. W. R. Wallace. 2006. MS Excel Program Developed for CO2 System Calculations. ORNL/CDIAC-105a. Carbon Dioxide Information Analysis Center, Oak Ridge National Laboratory, U.S. Department of Energy, Oak Ridge, Tennessee.

Renegar, D.A., Riegl, B.M., 2005. Effect of nutrient enrichment and elevated CO2 partial pressure on growth rate of Atlantic scleractinian coral Acropora cervicornis. Mar. Ecol. Prog. Ser. 293, 69-76.

Reynaud, S., Leclerq, N., Romaine-Lioud, S., Ferrier-Pages, C., Jaubert, J., Gattuso, J.-P., 2003. Interacting effects of $\mathrm{CO} 2$ partial pressure and temperature on photosynthesis and calcification in a scleractinian coral. Global Change Biol. 9, 1660-1668.

Rodrigues, L.J., Grottoli, A.G., 2006. Calcification rate and the stable carbon, oxygen, and nitrogen isotopes in the skeleton, host tissue, and zooxanthellae of bleached and recovering Hawaiian corals. Geochim Cosmochim Acta 70, 2781-2789.

Sakka, A., Legendre, L., Gosselin, M., LeBlanc, B., Delesalle, B., Price, N.M., 1999. Nitrate, phosphate, and iron limitation of the phytoplankton assemblage in the lagoon of Takapoto Atoll (Tuamotu Archipelago, French Polynesia). Aquat Microb Ecol 19, 149-161.

Schneider, K., Erez, J., 2006. The effect of carbonate chemistry on calcification and photosynthesis in the hermatypic coral Acropora eurystoma. Limnol. Oceanogr. 51, 1284-1293.

Tambutte, E., Allemand, D., Bourge, I., Gattuso, J.P., Jaubert, J., 1995. An improved $45 \mathrm{Ca}$ protocol for investigating physiological mechanisms in coral calcification. Marine Biol. 122, 453-459.

Tanaka, Y., Miyajima, T., Koike, I., Hayashibara, T., Ogawa, H., 2007. Imbalanced coral growth between organic tissue and carbonate skeleton caused by nutrient enrichment. Limnol. Oceanogr. 52, 1139-1146.

Vandermeulen, J.H., Muscatine, L., 1974. Influence of symbiotic algae on calcification in reef corals: critique and progress report. In: Vernberg, W.B. (Ed.), Symbiosis in the Sea pp.1-19. 
Table 1 . Average alkalinity $(\mu \mathrm{mol} / \mathrm{kg})$, DIC $(\mu \mathrm{mol} / \mathrm{kg})$, temperature $\left({ }^{\circ} \mathrm{C}\right)$ and salinity values for samples collected 2005-2009 from Great Harbor, Woods Hole, MA at $~ 1.5 \mathrm{~m}$ depth. Replicate alkalinity and DIC measurements differed by less than $11 \mu \mathrm{mol} / \mathrm{kg}$ except for samples collected 7/2/07, when reproducibility was better than $50 \mu \mathrm{mol} / \mathrm{kg}$.

$\begin{array}{ccccc}\text { Date } & \text { Temperature } & \text { Salinity } & \text { Alkalinity } & \text { DIC } \\ \text { 11/4/05 14:30 } & 13.1 & 30.73 & 2051 & 1877 \\ \text { 11/4/05 22:44 } & 13.0 & 30.76 & 2066 & 1883 \\ \text { 11/5/05 6:40 } & 12.9 & 30.88 & 2071 & 1893 \\ \text { 11/5/05 11:15 } & 12.9 & 30.66 & 2059 & 1874 \\ \text { 11/5/05 15:15 } & 12.9 & 30.77 & 2062 & 1880 \\ \text { 1/1/06 10:30 } & 4.7 & 30.50 & 2110 & 1957 \\ \text { 1/1/06 18:00 } & 4.7 & 30.50 & 2108 & 1948 \\ \text { 11/8/06 14:00 } & 11.6 & 31.81 & 2127 & 1961 \\ \text { 1/19/07 16:00 } & 4.9 & 31.11 & 2113 & 1967 \\ \text { 2/19/07 17:00 } & -0.8 & 31.93 & 2170 & 2007 \\ \text { 3/19/07 16:10 } & 2.6 & 31.58 & 2154 & 1995 \\ \text { 4/19/07 16:40 } & 6.2 & 31.52 & 2147 & 2001 \\ \text { 5/19/07 18:00 } & 12.4 & 31.59 & 2146 & 1982 \\ \text { 7/2/07 17:50 } & 20.1 & 31.58 & 2129 & 1922 \\ \text { 7/20/07 17:50 } & 22.1 & 31.46 & 2094 & 1935 \\ \text { 8/20/07 17:00 } & 21.8 & 31.54 & 2086 & 1918 \\ \text { 9/20/07 18:10 } & 19.9 & 31.85 & 2104 & 1930 \\ \text { 3/27/08 17:30 } & 5.4 & 31.00 & 2114 & 1981 \\ \text { 4/23/08 17:00 } & 9.6 & 31.33 & 2134 & 1974 \\ \text { 5/29/08 16:30 } & 14.4 & 31.19 & 2117 & 1958 \\ \text { 6/23/08 17:50 } & 20.1 & 31.41 & 2118 & 1949 \\ \text { 7/29/08 17:50 } & 24.2 & 31.21 & 2078 & 1865 \\ \text { 8/31/08 18:08 } & 21.8 & 31.57 & 2080 & 1893 \\ \text { 10/5/08 18:05 } & 17.8 & 31.30 & 2081 & 1898 \\ \text { 11/9/08 18:40 } & 12.8 & 31.58 & 2118 & 1936 \\ \text { 12/12/08 19:09 } & 7.0 & 31.22 & 2110 & 1960 \\ \text { 2/19/09 20:30 } & 1.9 & 31.25 & 2126 & 1987 \\ \text { 3/28/09 18:12 } & 4.7 & 31.45 & 2154 & 2017 \\ \text { 4/30/09 18:00 } & 11.0 & 31.32 & 2147 & 1992\end{array}$


Table 2. Carbonate speciation in each treatment. Values are calculated using average alkalinity, salinity and $\mathrm{pCO}_{2}$ values measured during the experiment. Constants of Mehrbach et al. (1973) as refit by Dickson and Millero (1987) are used for carbonate speciation, Dickson (1990) for sulfate. Units are $\mu \mathrm{mol} / \mathrm{kg}$ for carbonate species, alkalinity and $\mathrm{PO}_{4}, \mu$ atm for $\mathrm{pCO}_{2}$. An Excel implementation of CO2sys (Pierrot et al., 2006) was used for calculations.

\begin{tabular}{|l|l|l|l|l|l|l|l|l|l|l|}
\hline $\begin{array}{l}\text { treat- } \\
\text { ment }\end{array}$ & $\begin{array}{l}\mathrm{T} \\
\left({ }^{\circ} \mathrm{C}\right)\end{array}$ & $\mathrm{S}$ & $\mathrm{PO}_{4}$ & $\mathrm{Alk}$ & $\mathrm{pCO}_{2}$ & $\begin{array}{l}\mathrm{pH} \\
\text { total }\end{array}$ & $\mathrm{CO}_{2}$ & $\mathrm{HCO}_{3}$ & $\mathrm{CO}_{3}$ & $\begin{array}{l}\Omega \\
\text { aragonite }\end{array}$ \\
\hline ambient & $\begin{array}{l}25.8 \\
(.1)\end{array}$ & $\begin{array}{l}31 \\
(.9)\end{array}$ & $\begin{array}{l}0.5 \\
(.3)\end{array}$ & $\begin{array}{l}2110 \\
(40)\end{array}$ & $\begin{array}{l}390 \\
(10)\end{array}$ & 8.03 & 11 & 1662 & 182 & 3 \\
\hline $\mathrm{CO}_{2}$ & $\begin{array}{l}25.8 \\
(.1)\end{array}$ & $\begin{array}{l}31 \\
(.9)\end{array}$ & $\begin{array}{l}0.5 \\
(.3)\end{array}$ & $\begin{array}{l}2110 \\
(40)\end{array}$ & $\begin{array}{l}780 \\
(40)\end{array}$ & 7.78 & 22 & 1837 & 111 & 1.8 \\
\hline nutrient & $\begin{array}{l}25.8 \\
(.1)\end{array}$ & $\begin{array}{l}31 \\
(.9)\end{array}$ & $\begin{array}{l}0.7 \\
(.2)\end{array}$ & $\begin{array}{l}2110 \\
(40)\end{array}$ & $\begin{array}{l}390 \\
(10)\end{array}$ & 8.03 & 11 & 1662 & 182 & 3 \\
\hline $\begin{array}{l}\text { nutrient } \\
\& \mathrm{CO}_{2}\end{array}$ & $\begin{array}{l}25.8 \\
(.1)\end{array}$ & $\begin{array}{l}31 \\
(.9)\end{array}$ & $\begin{array}{l}0.7 \\
(.2)\end{array}$ & $\begin{array}{l}2110 \\
(40)\end{array}$ & $\begin{array}{l}780 \\
(40)\end{array}$ & 7.78 & 22 & 1837 & 111 & 1.8 \\
\hline
\end{tabular}

Table 3. Factorial ANOVA results, showing the significance of $\mathrm{CO}_{2}$, nutrients, and their interaction on rank transformed calcification rates with the parent coral colony included as a blocking variable

$\begin{array}{llllll}\text { Source } & \text { DF } & \text { SS } & \begin{array}{l}\text { Mean } \\ \text { Square }\end{array} & \mathrm{F} & \mathrm{p} \\ \text { Model } & 7 & 620.708 & 88.672619 & 2.68 & 0.0485 \\ \text { Error } & 16 & 529.291 & 33.080729 & & \\ \text { CO2 } & 1 & 216 & 216 & 6.53 & 0.0212 \\ \text { nutrients } & 1 & 4.16666 & 4.1666667 & 0.13 & 0.7273 \\ \text { CO2*nut } & 1 & 140.166 & 140.16666 & 4.24 & 0.0562 \\ \text { colony } & 4 & 260.375 & 65.09375 & 1.97 & 0.1482\end{array}$



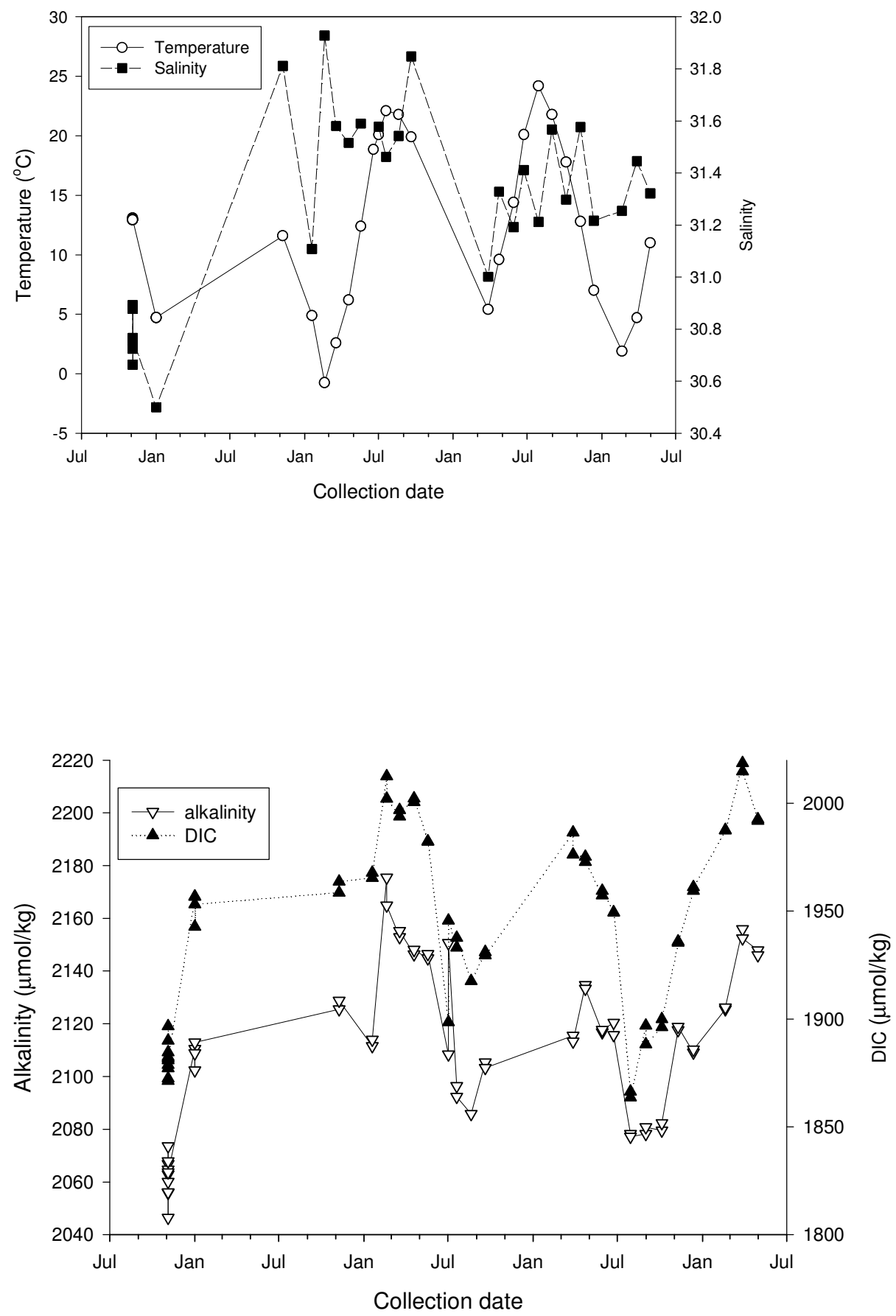
Figure 1. Temperature, salinity, alkalinity and DIC, values for samples collected 20052009 from Great Harbor, Woods Hole, MA. This shows the natural range in carbonate chemistry to which the A. poculata colonies used in this experiment are exposed. $\mathrm{T}$ and $\mathrm{S}$ are plotted in the upper plot, alkalinity and DIC in the lower. Points are individual measurements (usually 2 samples were taken at each time point). 


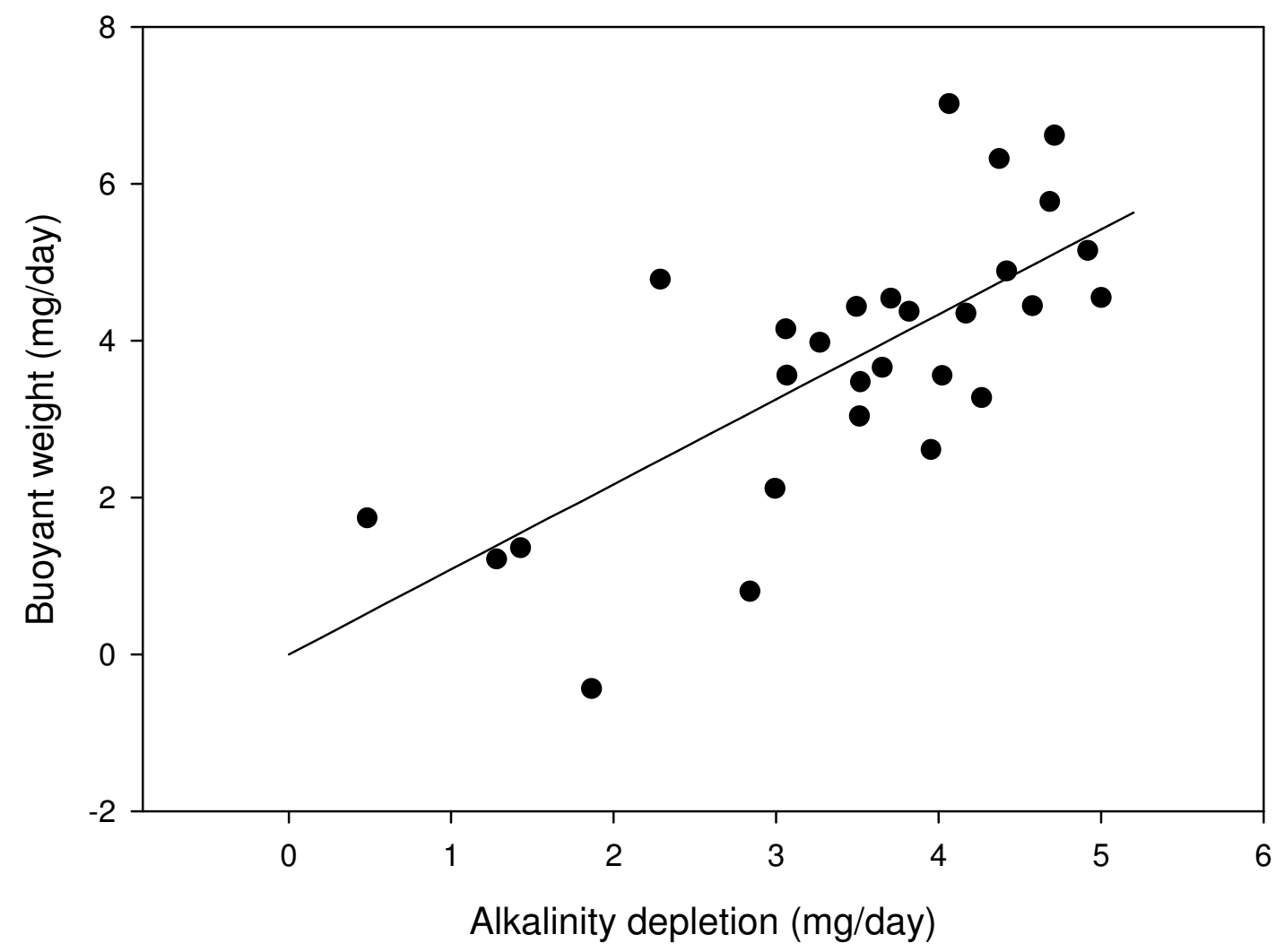

Figure 2. Relationship between growth rates based on alkalinity depletion and buoyant weight measurements. Each point represents a measurement for an individual pair of corals, the line represents the best fit line (buoyant weight $=1.084 *$ alkalinity depletion, $\mathrm{R}^{2}=0.523$ ). 


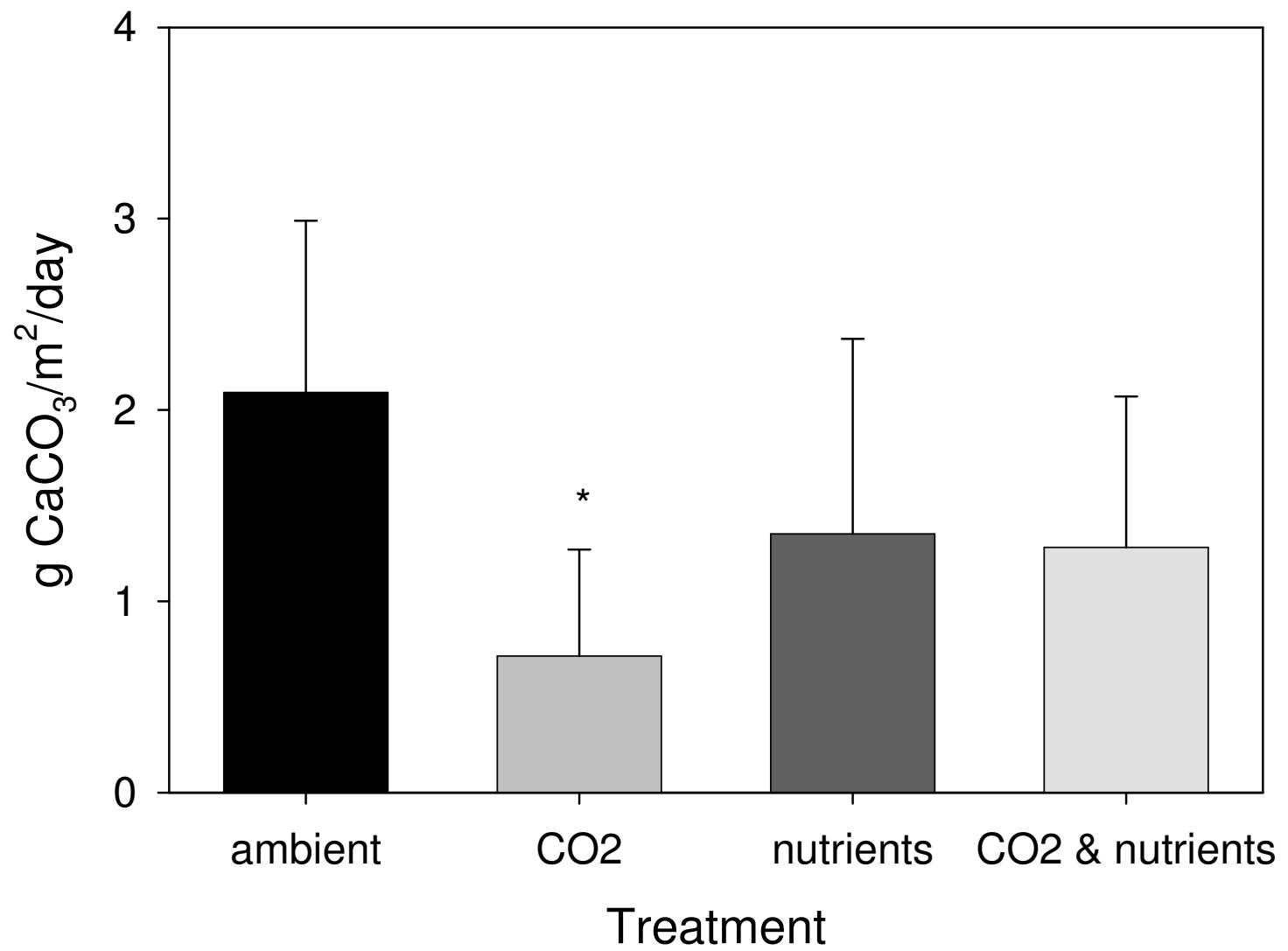

Figure 3. Average growth rates of zooxanthellate $A$. poculata colonies exposed to nutrient and $\mathrm{CO}_{2}$ treatments for six months. Bars represent means of six replicates, error bars are standard deviation. * indicates treatments significantly different from ambient $\mathrm{p}<0.05$. 


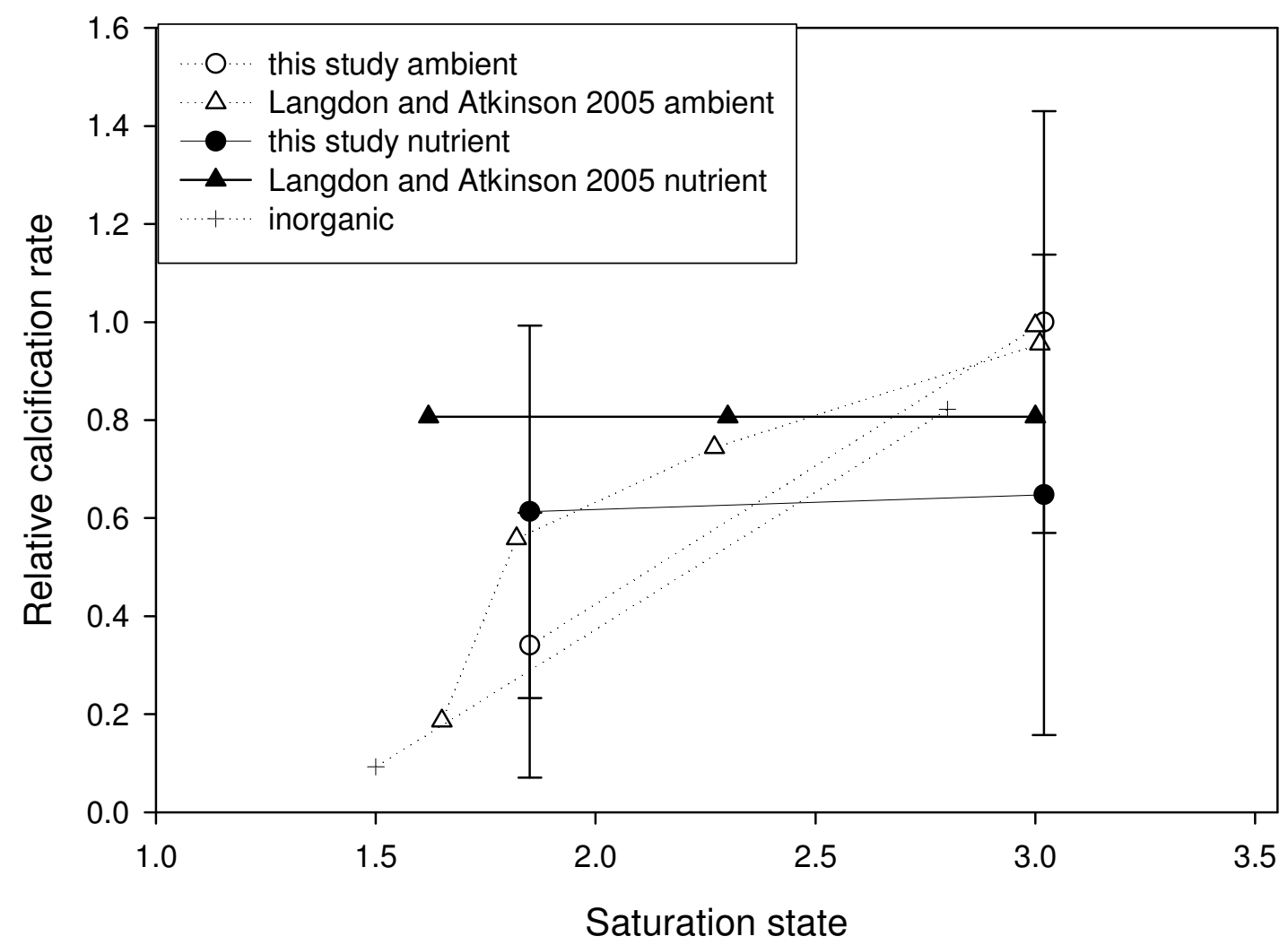

Figure 4. Comparison of relative calcification rates versus saturation state measured in the current study (circles) and values reported by Langdon and Atkinson (2005) (triangles). Corals are grown under either ambient nutrients (open symbols) or elevated nutrients (filled symbols). For clarity, error bars are only included for the current study. Inorganic aragonite growth data from Burton and Walter (1987) (cross) are included for reference purposes. Relative calcification rates are expressed as the ratio of the reported growth rate to the calculated growth rate under ambient conditions at a saturation state of 3 (values at 3 were calculated assuming a linear relationship between saturation state and growth using the 2 saturation states closest to 3 ). 


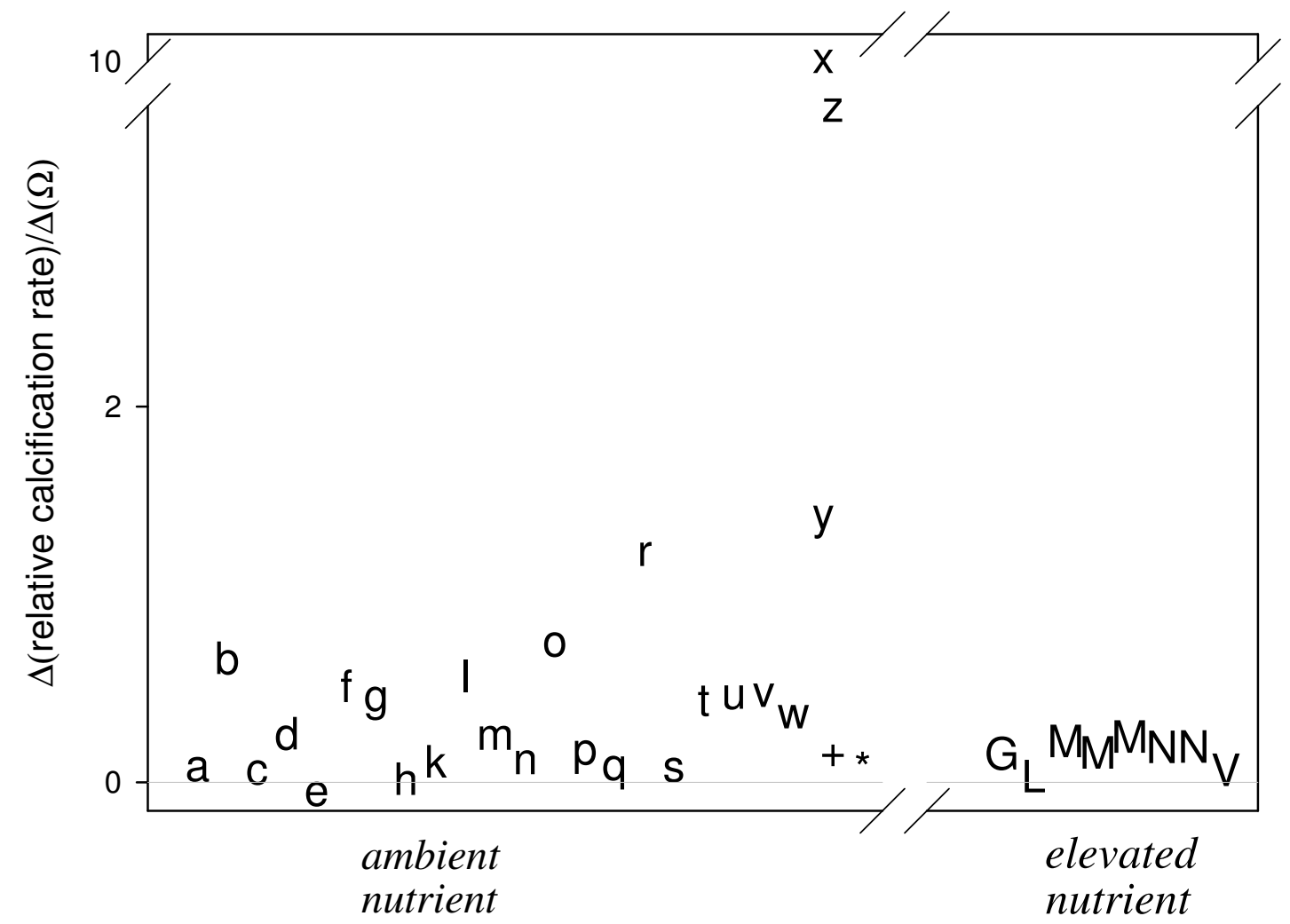

Figure 5. Changes in the relative calcification rate in response to saturation state for ambient and nutrient enriched conditions. Positions along the $\mathrm{x}$-axis within the ambient and nutrient enriched groups are arbitrary and intended only to separate symbols. Y-axis values are slopes from linear regressions of relative calcification rate versus saturation state determined for each study. Higher Y values indicate a positive slope, and thus decreasing calcification rate with decreasing saturation state. Near-zero Y values indicate little response of calcification to lowered saturation state. Each letter represents a different study: letters a-l represent studies in which $\mathrm{CO}_{2}$ was manipulated, the remainder used acid/base and/or bicarbonate addition. Some studies used multiple nutrient levels, different levels are represented by the same letter, not all studies included nutrients as a treatment and are thus plotted at ambient nutrients only. Most studies showed significant correlations $(\mathrm{p}<0.1)$ between relative calcification rate and saturation state, studies with correlations of $p>0.3$ are excluded (using a $p$ value of 0.3 allows inclusion of studies $h$ and $\mathrm{k}$ which were not well fit by a linear relationship but for which there was a response to saturation state). When data ranges were given instead of average values, the minimum and maximum were averaged for calculations. Studies are as follows: $\mathrm{a}=$ Leclerq et al., 2000 light, b = Leclerq et al., 2000 dark, c = Leclerq et al., 2002 light, d = Leclerq et al., 2002 dark, e = Reynaud et al., 2003 25C, f = Reynaud et al., 2003 28C, g = Renegar and Riegl, 2005, h = Anthony et al., 2008 Porites sp. $\sim 25.5^{\circ} \mathrm{C}, \mathrm{i}=$ Anthony et al., 2008 Porites sp. $\sim 28.5^{\circ} \mathrm{C}, \mathrm{j}=$ Anthony et al., 2008 Acropora sp. $\sim 28.5^{\circ} \mathrm{C}, \mathrm{k}=$ 
Anthony et al., 2008 Acropora sp. $\sim 25.5^{\circ} \mathrm{C}, 1=$ This study, $\mathrm{m}=$ Marubini and Atkinson, 1999, $\mathrm{n}=$ Marubini and Thake, 1999, o = Langdon et al., 2000, $\mathrm{p}=$ Marubini et al., 2001, q = Marubini et al., 2001, $\mathrm{r}=$ Langdon et al., 2003, s = Marubini et al., 2003, $\mathrm{t}=$ Ohde and Hossain, 2004 dark, $\mathrm{u}=$ Ohde and Hossain, 2004 light, $\mathrm{v}=$ Langdon and Atkinson, 2005, w = Schneider and Erez, 2006, $\mathrm{x}=$ Schneider and Erez, 2006 dark, $\mathrm{y}=$ Herfort et al., 2008 Acropora sp. dark, $\mathrm{z}=$ Herfort et al., 2008 Acropora sp. light, $+=$ Herfort et al., 2008 Porites sp., * = Marubini et al., 2008. 


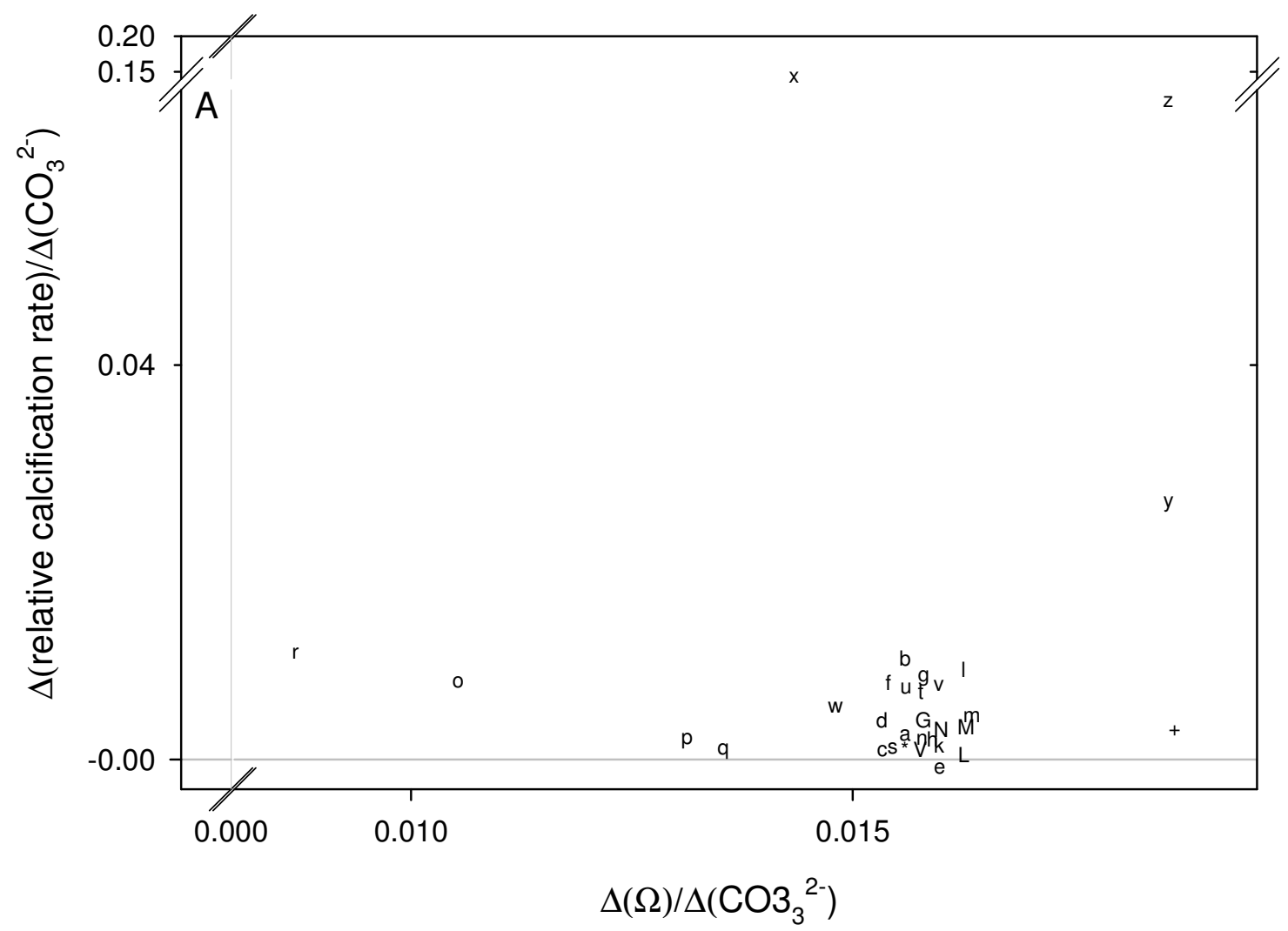




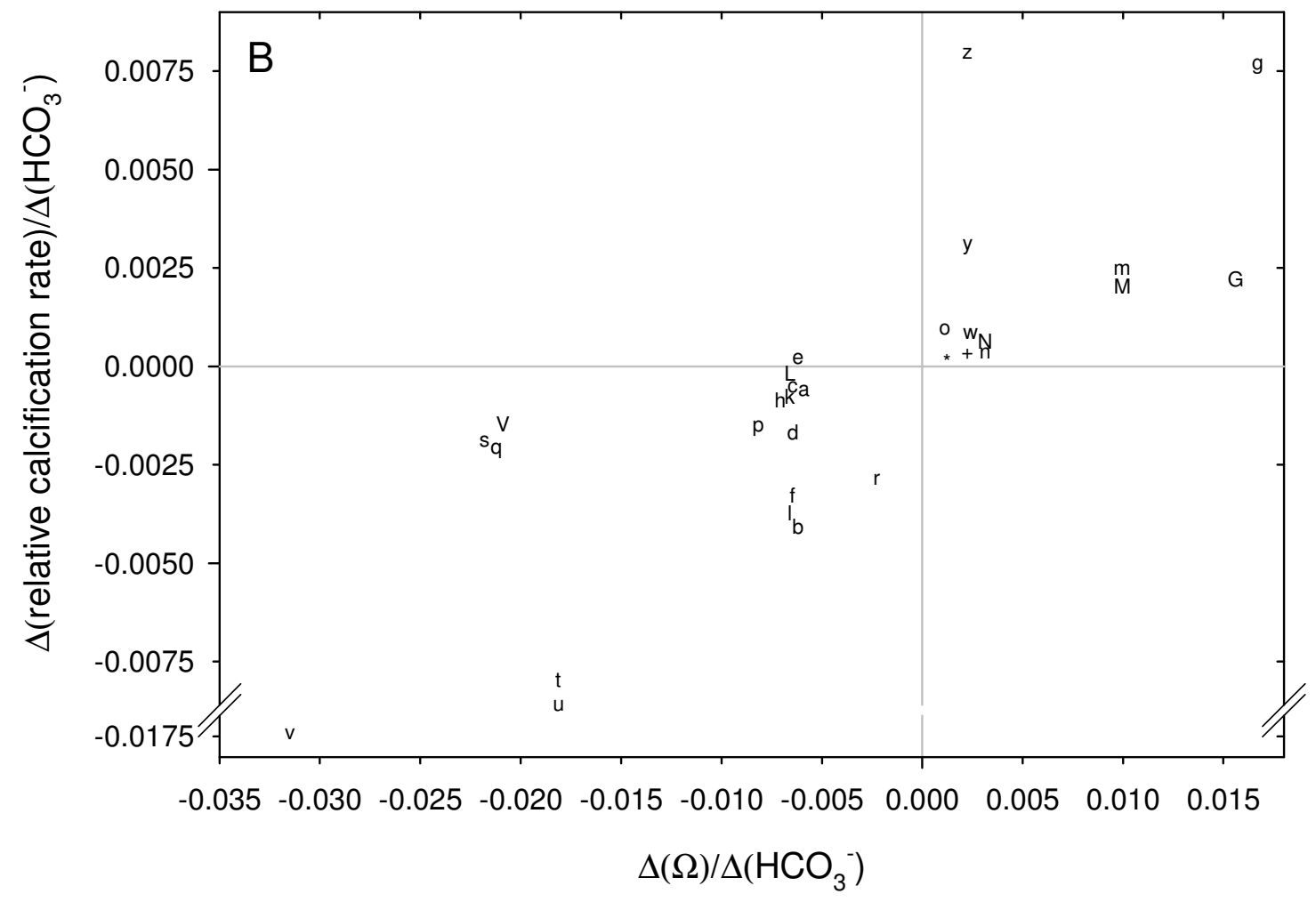




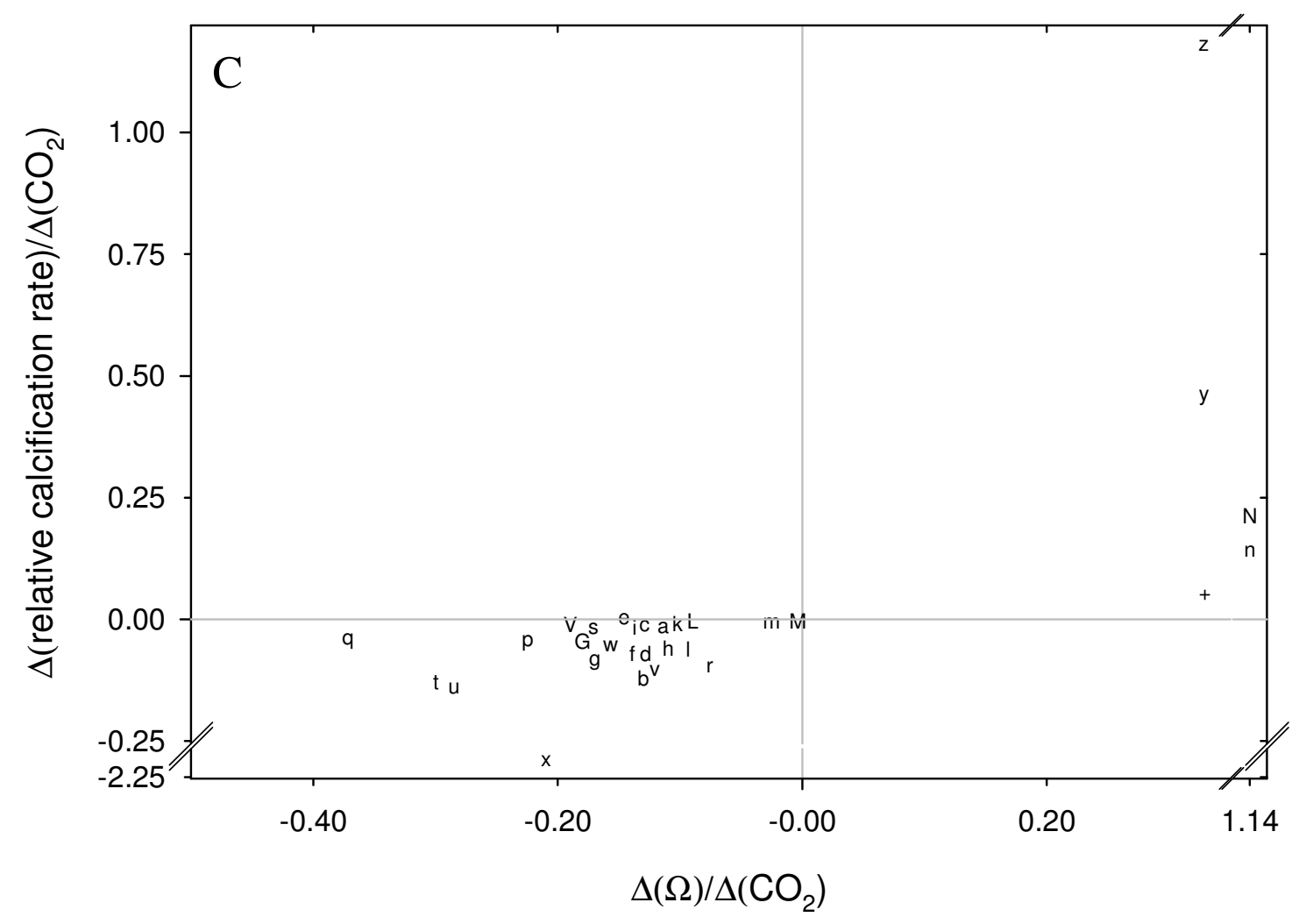




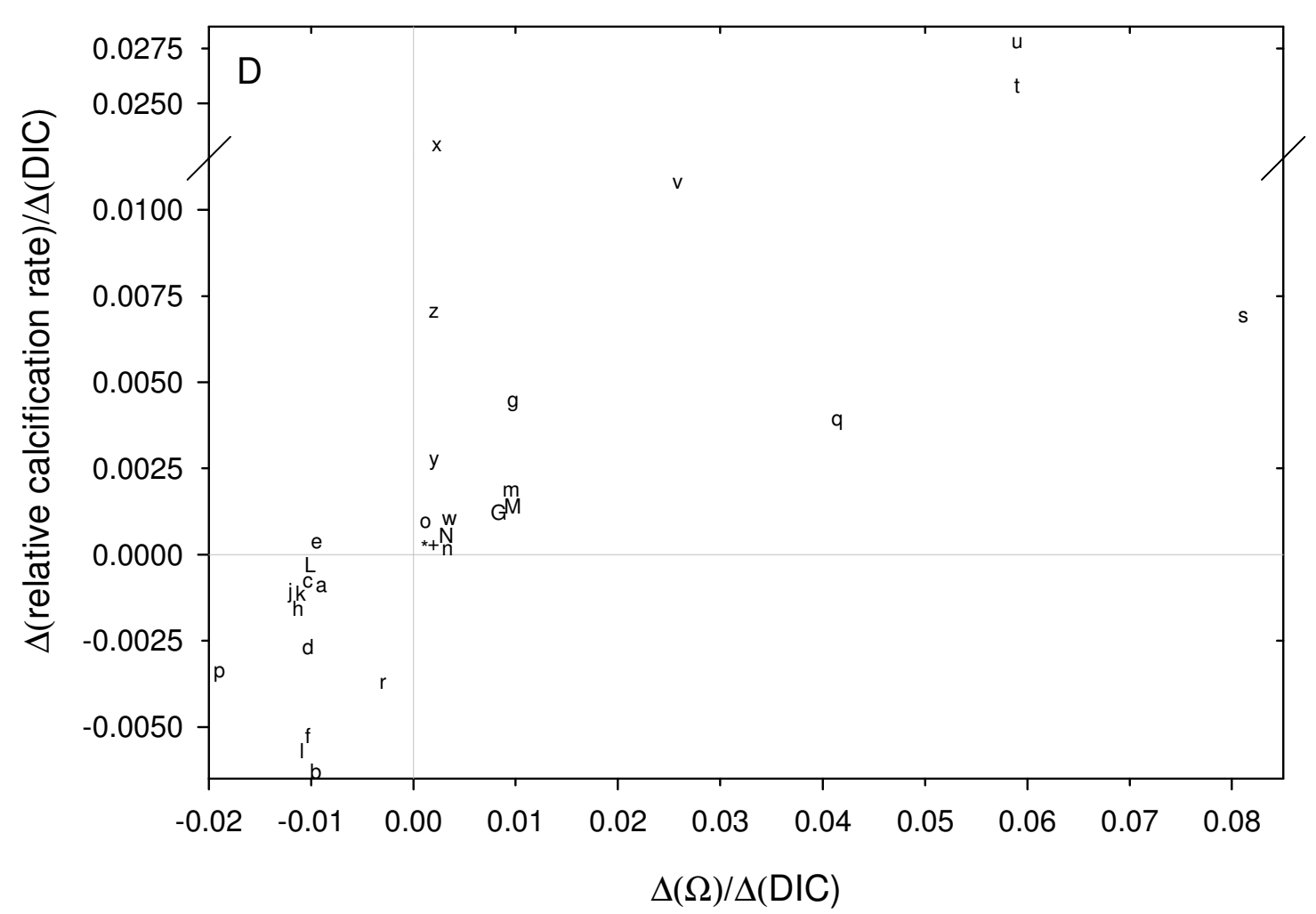

Figure 6. Slope-slope plots of changes in the relative calcification rate as a function of the concentration of carbonate species (carbonate ion, bicarbonate ion, aqueous $\mathrm{CO}_{2}$ and total DIC), versus the change in saturation state as a function of the carbonate species concentration. A. Change in the relative calcification rate in response to carbonate ion versus the change in saturation state with carbonate ion. Note that in all cases, saturation state and carbonate-ion concentrations are positively correlated, and in all but one case, calcification is positively correlated with carbonate-ion. As a result, all but one study falls in the upper right quadrant. This pattern is consistent with calcification rate being controlled by either carbonate ion concentration or saturation state. B. Changes in the relative calcification rate in response to bicarbonate ion versus the change in saturation state with bicarbonate ion. Note that in all cases in which saturation state and bicarbonate-ion concentrations are positively correlated, calcification is positively correlated with bicarbonate-ion (points fall in the upper right quadrant). However, when saturation state and bicarbonate are negatively correlated, calcification is generally negatively correlated with bicarbonate (points fall in lower left quadrant). This pattern is not consistent with a bicarbonate ion concentration control on calcification rate, instead saturation state appears to be the controlling factor. C. Changes in the relative calcification rate in response to aqueous $\mathrm{CO}_{2}$ versus the change in saturation state with aqueous $\mathrm{CO}_{2}$. Note that in all cases in which saturation state and aqueous $\mathrm{CO}_{2}$ concentrations are positively correlated, calcification is positively correlated with 
aqueous $\mathrm{CO}_{2}$ (upper right quadrant). When saturation state and aqueous $\mathrm{CO}_{2}$ are negatively correlated, calcification is generally negatively correlated with aqueous $\mathrm{CO}_{2}$ (lower left quadrant). As with the bicarbonate data, this pattern suggests the key role of saturation state in controlling calcification rate. D. Changes in the relative calcification rate in response to DIC versus the change in saturation state with DIC. Here too, the pattern suggests that saturation state, and not DIC concentration per se, controls calcification rate. Y-axis values are slopes from linear regressions of relative growth rate versus the given carbonate species determined for each study. X-axis values are from linear regressions of saturation state versus the given carbonate species. For carbonate species not listed in the original publication, concentrations were calculated using CO2sys (Pierrot et al., 2006), with the reported measured values as inputs. The constants of Mehrbach et al. (1973) are used for carbonate speciation, Dickson (1990) for sulfate. All plotted studies showed correlations $(\mathrm{p}<0.3)$ between relative calcification rate and the given carbonate species and between saturation state and the given carbonate species. Overlapping symbols are offset. Each letter represents a different study as in Figure 5. Nutrient enriched conditions are indicated by a capital letter. 
Chapter 4: Coral gender: an unexplored factor in coral calcification?

\begin{abstract}
Zooxanthellate and azooxanthellate colonies of the scleractinian coral Astrangia poculata were grown for $\sim 1$ year at near constant temperature $\left(16^{\circ} \mathrm{C}\right.$ or $\left.24^{\circ} \mathrm{C}\right), 4$ months of which were at altered $\mathrm{pCO}_{2}$ and nutrient $(\mathrm{N}, \mathrm{P}, \mathrm{Fe})$ levels. Calcification rates, as estimated from buoyant weights, were negatively affected by $\mathrm{CO}_{2}$ enrichment for zooxanthellate corals grown at $24^{\circ} \mathrm{C}$. No significant nutrient effect was found, however nutrient levels were high in the source seawater throughout the experiment. No signficant $\mathrm{CO}_{2}$ effect was found under other temperature or symbiont conditions for buoyant weight based calcification rates, though values tended to be lower for $\mathrm{CO}_{2}$ treated corals. Such results appear contradictory to a calcification model in which zooxanthellae allow the coral to benefit from elevated $\mathrm{CO}_{2}$ levels, but may reflect relatively low symbiont densities. Gamete release was observed at several time points over the course of the experiment allowing coral gender to be established. Regardless of treatment, calcification rates were significantly higher in males and reduced calcification rates associated with $\mathrm{CO}_{2}$

treatment were more frequent in females. This result suggests coral gender may be an important factor influencing calcification rate which needs further exploration.
\end{abstract}

Note: additional techniques related to this chapter and alternate presentations of the data are found in Appendix 4. Additional data documenting environmental conditions, and measured physiological parameters are presented as well. Large errors associated with some of the additional physiological parameters limits interpretation, thus they are not included as part of the chapter. Appendix 5 and 6 include a paper submitted to Nature based on data presented in this chapter - nutrient and zooxanthellate levels are combined in the Nature paper to provide enough replicates to test for an effect of gender. 


\section{Introduction}

Ocean acidification and the associated decline in the aragonite saturation state has been suggested to be one of the major threats to coral reefs globally (e.g., Kleypas et al., 1999). However, experimental studies examining the effects of ocean acidification on coral calcification show mixed results, with many showing negative effects (e.g., Marubini and Atkinson 1999; Langdon and Atkinson 2005), and a few showing neutral to positive effects (Reynaud et al., 2003; Anthony et al., 2008; Jury et al., 2009). Nutrient availability has been suggested as one factor which may influence how corals respond to acidification (e.g., Holcomb et al., submitted). Nutrient enrichment under ambient $\mathrm{CO}_{2}$ conditions may lead to calcification being limited by carbon availability due to competition with the symbiotic zooxanthellae for a limited supply of $\mathrm{CO}_{2}$ (e.g., Marubini and Davies 1996). Under such $\mathrm{CO}_{2}$ limited conditions, an elevation of $\mathrm{CO}_{2}$ could enhance calcification. Some data appear consistent with such a model (Langdon and Atkinson 2005; Holcomb et al., submitted); however, a number of key tests remain to be run. Central to the $\mathrm{CO}_{2}$ limitation model is competition between zooxanthellae and calcification for carbon, thus azooxanthellate corals would not be expected to respond positively to elevated $\mathrm{CO}_{2}$ under nutrient enriched conditions, and calcification should respond negatively to declining saturation state.

The temperate coral, Astrangia poculata provides an ideal organism for testing the $\mathrm{CO}_{2}$ limitation model as it occurs naturally with and without symbiotic zooxanthellae (Boschma 1925). Thus, the interaction between nutrients and $\mathrm{CO}_{2}$ can be examined in zooxanthellate and azooxanthellate colonies of the same species. To accomplish this, 
zooxanthellate and azooxanthellate colonies of Astrangia poculata were grown at two temperatures under four combinations of nutrients and $\mathrm{CO}_{2}$ : ambient seawater, nutrient enriched seawater, $\mathrm{CO}_{2}$ enrichment, and nutrient and $\mathrm{CO}_{2}$ enrichment.

\section{Methods}

\section{Coral collection}

Four azooxanthellate (white) colonies of Astrangia poculata were collected September 19, 2008 at a depth of $3 \mathrm{~m}$ from beneath the Woods Hole Oceanographic Institution pier (Woods Hole, MA USA) at a site far from the edges of the pier (and thus never exposed to direct sunlight). Four zooxanthellate (brown) colonies were collected September 25, 2008 at a depth of 3-6m from one of the outer pier pilings (exposed to direct sunlight).

\section{Initial processing}

On the day of collection corals were returned to lab, fragmented with wire cutters into at least 11 pieces; bored regions, worms, crabs, sponges and other organisms were removed. Once trimmed to size, each fragment was placed into a plastic cup suspended below a balance and weighed (see buoyant weight procedure below). Nine fragments from each colony were attached to individual acrylic slides with cyanoacrylate adhesive (EZ bond). Each acrylic slide measured $\sim 2.5 \mathrm{~cm}$ by $\sim 5 \mathrm{~cm}$ with blue acrylic pieces attached (using acrylic solvent cement) near each corner to provide photographic 
reference points for measurement purposes. Each slide had been engraved with a unique identifier, weighed, and soaked repeatedly in both seawater and freshwater prior to use.

Once attached to slides, the cyanoacrylate was allowed to cure for one day before photographs were taken, and the fragments (with slide) were weighed and assigned to individual aquariums. At least one fragment from each parent colony was assigned to each aquarium. All aquariums were initially at ambient conditions. To minimize stress to the coral, corals were handled underwater to the extent possible. Filtered ambient Vineyard Sound (Woods Hole, MA USA) seawater was used for all initial processing steps; temperature ranged from $21-21.6^{\circ} \mathrm{C}$.

One fragment from each coral was placed in $95 \%$ ethanol and stored at $-20^{\circ} \mathrm{C}$. Another fragment was used for initial tissue sampling (see Appendix A4).

\section{Aquarium set up}

Water supply

Coral fragments were maintained in a flow-through aquarium system. Two 450L reservoirs were filled daily with Vineyard Sound seawater $(20 \mu \mathrm{m}$ filtered); water from a given reservoir was continuously pumped to a header tank. From each header tank, water fed into gas mixing chambers to equilibrate the water with a $\mathrm{CO}_{2} /$ air mixture, and from there into valve manifolds used to deliver water to each aquaria. Prior to entering each aquarium, water first passed through a coil of tubing held within the water bath holding the aquaria to allow for temperature equilibration, then entered a small gas mixing chamber held within the water bath to allow for $\mathrm{CO}_{2}$ equilibration at the experimental 
temperature. Total flow through all tanks supplied by each reservoir are shown in Figure A-1a; approximately $30-40 \%$ of the total flow went to tanks held at $16^{\circ} \mathrm{C}$ and the remainder to tanks held at $24^{\circ} \mathrm{C}$, turnover rates were greater than once per hour for all tanks. Lower flow rates were used at $16^{\circ} \mathrm{C}$ to improve temperature stability. Periodically over the course of the experiment, Ba isotope spikes were added to each reservoir to mark the skeleton deposited at a particular point in time, each spike nearly doubled the total $\mathrm{Ba}$ concentration at the time of addition.

\section{Aquaria}

Individual aquaria consisted of $43 \times 5.5 \mathrm{~cm}$ PETG containers filled to $8 \mathrm{~cm}$ depth. Half of each container was exposed to light (for zooxanthellate corals), while the other half was kept dark (for azooxanthellate corals). The outside of each container was colored black (using permanent marker) to reduce stray light. Each container was divided into two sections by a piece of opaque blue acrylic placed $\sim 21 \mathrm{~cm}$ from the end and $\sim 2 \mathrm{~cm}$ from the bottom and continuing to the surface. Containers were covered with PETG lids, one half of each lid was covered with blue acrylic to block light from entering the dark half of the container (to ensure azooxanthellate colonies remained azooxanthellate). Each container had two water inlets, three air inlets and two water outlets. Water inlets and outlets were placed at each end of the container and the flow direction altered every $\sim 8 \mathrm{~d}$ such that half the time water entered at the front of the tank and exited at the back, while the remainder of the time water entered at the back and exited at the front. Air inlets supplied air to aquarium air-stones placed at the front, 
middle, and back of each aquarium. A total of 8 aquaria were used, four in each of two temperature controlled water baths.

\section{Temperature}

Following a gradual transition from ambient to experimental temperatures, one water bath was set at $16^{\circ} \mathrm{C}$, the other at $24^{\circ} \mathrm{C}$. At $24^{\circ} \mathrm{C}$, the seasonal maximum temperature in Great Harbor, MA, calcification and photosynthesis rates are expected to be at their seasonally highest values, and photosynthesis is expected to enhance growth (e.g. Cummings 1983; Jacques and Pilson 1983). At $16^{\circ} \mathrm{C}$, the contribution of photosynthesis is expected to be negligible, and growth rates should be similar for both symbiotic and asymbiotic colonies (e.g. Cummings 1983; Jacques and Pilson 1983). Temperature stability was monitored using digital thermometers (VWR) to record daily minimum and maximum temperatures, periodic manual checks with a mercury thermometer (calibration checked against a Super-Thermometer II (Hart Scientific) and high precision temperature bath maintained by the WHOI CTD facility), and temperature loggers to record temperature every $12 \mathrm{~min}$ (U12 logger, TMC-HD probe, Onset Inc.), temperature logs are shown in Figure A-1b , total temperature variation is $0.8^{\circ} \mathrm{C}$ about the set point (except for a brief period when the $24^{\circ} \mathrm{C}$ bath was emptied for replacement of a chiller and temperature dropped by $\sim{ }^{\circ} \mathrm{C}$ ) with typical daily variations of $0.15^{\circ} \mathrm{C}$ for the $24^{\circ} \mathrm{C}$ bath and $0.5^{\circ} \mathrm{C}$ for the $16^{\circ} \mathrm{C}$ bath. Each water bath was heated with aquarium heaters controlled by an Omega CN1504 controller using aquarium heaters and thermistor temperature probes, thermo-electric chillers (Ice-probe, Coolworks) were used 
to chill the $24^{\circ} \mathrm{C}$ temperature bath, and a compressor based chiller (Prime, Current USA) used for the $16^{\circ} \mathrm{C}$ bath. Aquarium power-heads were used to continually circulate water within each bath.

Each aquarium in the $16^{\circ} \mathrm{C}$ water bath contained 4 zooxanthellate corals, 4 azooxanthellate corals, and 2 slides without corals. Each aquarium in the $24^{\circ} \mathrm{C}$ water bath contained 5 zooxanthellate corals, 5 azooxanthellate corals, and 2 slides without corals.

\section{Lighting}

Lighting was provided by two T5-HO bulbs (2 10000K bulbs, 48" 54w), on a $12 \mathrm{hr} 1 / \mathrm{d}$ cycle; light levels varied across the length of the bulb such that aquaria near the center of the bulb received more light than those toward the end. Light levels experienced by the light half of each aquarium are tabulated in Table A-1.

\section{Feeding}

Corals were fed daily. Each day freshly hatched brine shrimp (hatched from OSI brand eggs) were concentrated in a $150 \mu \mathrm{m}$ sieve, mixed with fresh seawater, and the shrimp were allowed to concentrate near a light source. Shrimp were added through two holes in the lid of each aquarium, one hole was on each side of the divider at the center of each aquarium, and half the aliquot of shrimp was added through each hole. A total of $6 \mathrm{ml}$ of shrimp suspension was added to the $16^{\circ} \mathrm{C}$ aquaria, $9 \mathrm{ml}$ to the $24^{\circ} \mathrm{C}$ aquaria. Periodically, $1 \mathrm{ml}$ samples of shrimp suspension were taken, filtered through a pre- 
combusted $\left(10 \mathrm{hr}\right.$ at $\left.475^{\circ} \mathrm{C}\right)$, pre-weighed $0.7 \mu \mathrm{m}$ glass fiber filter (Fisher), and dried at $40^{\circ} \mathrm{C}$. The average dry weight of shrimp suspension was $8 \mathrm{mg} / \mathrm{ml}$ (standard deviation: 3 $\mathrm{mg}, \mathrm{N}=15)$.

\section{Nutrients}

During the treatment phase of the experiment, nutrient levels were elevated by adding $\mathrm{NaNO}_{3}, \mathrm{~K}_{2} \mathrm{HPO}_{4}$, and $\mathrm{FeCl}_{2}$ stock solutions at a ratio of $2 \mu$ stock solution/L seawater to one of the reservoirs to increase concentrations of $\mathrm{NO}_{3}{ }^{-}, \mathrm{PO}_{4}{ }^{-3}$, and $\mathrm{Fe}^{+2}$ by 5 $\mu \mathrm{M}, 0.3 \mu \mathrm{M}$ and $2 \mathrm{nM}$ (per Chapter 3). Measured values were: $0.3 \pm 0.1 \mu \mathrm{M} \mathrm{NH}_{4}, 1.6 \pm 0.6$ $\mu \mathrm{M} \mathrm{NO}_{3}, 0.1 \pm 0.2 \mu \mathrm{M} \mathrm{PO}_{4}$ in the source water prior to the start of treatments, $0.5 \pm 0.3 \mu \mathrm{M}$ $\mathrm{NH}_{4}, 2.9 \pm 0.8 \mu \mathrm{M} \mathrm{NO}_{3}, 0.5 \pm 0.1 \mu \mathrm{M} \mathrm{PO}_{4}$ for ambient seawater during treatment, and $0.4 \pm 0.1 \mu \mathrm{M} \mathrm{NH}_{4}, 8.1 \pm 1.7 \mu \mathrm{M} \mathrm{NO}_{3}, 0.8 \pm 0.2 \mu \mathrm{M} \mathrm{PO}_{4}$ for nutrient treated seawater. Nutrient levels were monitored both within reservoirs and within individual tanks by taking water samples every $\sim 8$ days throughout the experiment. Each sample was filtered through a syringe filter $(0.45 \mu \mathrm{m})$ into HDPE scintillation vials with foamed polyethylene lid liners (Wheaton) and stored frozen until measured. Vials used for nutrient sampling were soaked for at least $12 \mathrm{hr}$ in distilled water, rinsed repeatedly and air dried prior to use; $10-20 \mathrm{ml}$ of sample was used to rinse the syringe filter prior to sample collection. Samples were analyzed for ammonium, silicate, phosphate, and nitrate/nitrite (expressed as $\mathrm{NO}_{3}$ ) by the WHOI Nutrient Analytical Facility (using a Lachat Instruments QuickChem 8000 flow injection system). Nutrient data are summarized in Table 1 and are plotted over time in Figure A-1c-f. 
$\mathrm{CO}_{2}$

During the treatment phase of the experiment $\mathrm{CO}_{2}$ levels were increased for one set of gas mixing chambers and their corresponding aquaria using ambient air (provided by a Porter Cable oil free air-compressor) and $\mathrm{CO}_{2}$. Flow rates for $\mathrm{CO}_{2}$ and air were controlled by rotameters (Alborg Instruments), to provide an air/ $\mathrm{CO}_{2}$ mixture with $\mathrm{CO}_{2}$ levels 400 ppm above ambient. A Qubit s151 $\mathrm{CO}_{2}$ analyzer and a commercially prepared $\mathrm{CO}_{2}$ standard (Corp Brothers) were used daily to assess the stability of $\mathrm{CO}_{2}$ levels (Fig. A-1g). A dial barometer (Fisher) was used to measure atmospheric pressure at the time of each $\mathrm{CO}_{2}$ measurement (Fig. A-1g). Gas flow rates to all aquaria were $\sim 1$ $\mathrm{L} / \mathrm{min}$.

\section{Chemistry}

Water samples for nutrients (see above), alkalinity, $\mathrm{pH}, \mathrm{d}^{18} \mathrm{O} \mathrm{H}_{2} \mathrm{O}, \mathrm{DIC}, \mathrm{d}^{13} \mathrm{C}$ DIC, cations, and salinity were collected every $\sim 8$ days during the daytime throughout the experiment. Oxygen was measured occasionally as well (both during the day and at night). Following sampling, tanks were cleaned of algal growth using a credit card to scrape algae from the sides of the tanks and a pipet to remove algae and detritus from the tank, flow directions were switched and rates adjusted if needed. Details on chemistry sampling and associated data are included in Appendix A4. Average alkalinity, temperature, salinity, nutrient, and $\mathrm{pH}_{\mathrm{T}}$ values were used to calculate carbonate and nutrient chemistry for each aquarium before and after the start of treatments (Table 1). 


\section{Buoyant Weights}

Buoyant weight measurements (e.g. Davies 1989) were made every 1-2 months. A Sartorius GC803S balance (resolution $0.2 \mathrm{mg}$, reproducibility of standards was better than 5mg) with weigh-below hook was used to weigh corals. Glass and aluminum weights were used to estimate water density. Temperature was recorded at the time of every measurement and controlled using an Omega cn1504 temperature controller and aquarium heater, chilled seawater $\left(\sim 10^{\circ} \mathrm{C}\right)$ was added as needed to keep the temperature down for corals grown at $16^{\circ} \mathrm{C}$. Seawater was at ambient conditions for all buoyant weight measurements, only temperature was changed to match the treatment temperature. Salinity was recorded once to twice over the course of each weighing session and used to calculate expected seawater density (Fofonoff 1985). Mass changes are assumed to reflect changes in the mass of aragonite, an aragonite density of $2.9 \mathrm{~g} / \mathrm{cm}^{3}$ was used to calculate the dry weight of calcium carbonate deposited. Buoyant weight measurements were corrected for the contribution of the acrylic slide and cyanoacrylate using a density of $1.17 \mathrm{~g} / \mathrm{cm}^{3}$ for the slide and adhesive. The mass of the slide with adhesive was estimated from the buoyant weight change between the initial weighing of the unmounted coral fragment and the mounted weight the following day, coral growth was assumed to be negligible during this interval.

\section{Gender identification}


Genders were established either by direct observation of gamete release, or by verifying the presence of sperm in incubations of individual specimens in which the water had become cloudy. Direct observation of spawning was made when corals were weighed. During most weighing sessions, some corals spawned allowing for the gamete type released by individual polyps to be identified. Spawning males were readily identified as specimens tend to release sperm in several pulses providing several opportunities to observe gamete release. Female identification was difficult as fewer eggs were released, generally in one or two pulses. Only those specimens observed as they were releasing eggs could be identified as female. In individual incubations carried out for alkalinity depletion and oxygen consumption measurements (see Appendix A4), spawning males often released sperm in sufficient quantity to cloud the water and microscopic examination of water samples from individual incubations which had become cloudy allowed for the identification of sperm and establishment of gender. Dates of spawning observations are given in Table A-3.

\section{Statistics}

Linear regressions, used to determine pre-treatment and treatment calcification rates were performed using Systat. Pre-treatment rates were determined using weights from the first set of measurements after treatment temperatures were reached to the last set of measurements prior to the start of treatments. Treatment rates were based on the first set of weights once treatment conditions were reached through the final set of measurements. To test for an effect of gender, overall pre-treatment buoyant weight 
based calcification rates were analyzed using an ANOVA with the individual parent colony nested within gender. Effects of treatments $\left(\mathrm{CO}_{2}\right.$ and nutrient) were assessed using a factorial ANOVA model, including the gender and the parent colony nested within gender as blocking variables. Each temperature and symbiotic state was analyzed separately. To test for treatment effects, all data were expressed as differences between the treatment and pre-treatment rates relative to the pre-treatment rate for ANOVA analysis. ANOVAs were run using SAS. All data are given as the mean with standard deviation in parenthesis unless otherwise noted.

\section{Results}

\section{Aquarium conditions}

Conditions in all tanks were similar during the initial phase, and consistent differences between treatment conditions were maintained throughout the treatment period (Table 1 ). Daily $\mathrm{pH}$ variations of $\sim 0.1$ were experienced by all tanks, with lower values at night, however $\mathrm{CO}_{2}$ treatments consistently had lower $\mathrm{pH}$ values than tanks not treated with $\mathrm{CO}_{2}$, and the difference between treatments was greater than the daily cycle. Source water alkalinity was lower and nutrient levels higher during the treatment period relative to the pre-treatment phase. The rise of ambient nutrient levels during the treatment phase $\left(\sim 0.4 \mu \mathrm{M}\right.$ for $\left.\mathrm{PO}_{4}{ }^{3-}, 1.3 \mu \mathrm{M} \mathrm{NO}_{3}{ }^{-}\right)$relative to pre-treatment conditions exceeded desired treatment elevations for $\mathrm{PO}_{4}{ }^{3-}$, and represented 1/4 of the desired treatment elevation for $\mathrm{NO}_{3}{ }^{-}$. Values reported in Table 1 should reflect the maximum day-time variability in water chemistry and should be biased toward lower $\mathrm{pCO}_{2}$ values 
as all measurements were made shortly before tanks were cleaned of algal growth, airstones replaced, and water flows adjusted (usually increased as flows tended to drop between adjustments). Periods in which low water flow rates to a given tank occurred are characterized by low alkalinity values, as can be seen in Figure A-1H. Saturation state variations associated with water flow induced alkalinity variations were small relative to treatment differences (Table 1).

\section{Gender}

Gamete release was observed several times over the course of the experiment. All specimens observed spawning from a given parent colony spawned as the same gender, and gender was not observed to change over time. Fragments from 5 of the parent colonies spawned as males ( 2 azooxanthellate, 3 zooxanthellate). Fragments from 3 of the parent colonies spawned as females ( 2 azooxanthellate, 1 zooxanthellate). Not all fragments from a given parent colony were observed releasing gametes, and gamete release was never observed in corals grown at $16^{\circ} \mathrm{C}$. For corals which were not observed spawning, gender assignments are based on the gamete type released by fragments obtained from the same parent colony which spawned. Eggs were $\sim 120 \mu \mathrm{m}$ in diameter. Observations are detailed in Table A-3.

\section{Calcification rates}

Buoyant weight data show all corals to be growing throughout the experiment (Fig. A- 3). Overall pre-treatment growth rates were 3(1) mg/d (expressed as mean and 
standard deviation) for zooxanthellate corals at $24^{\circ} \mathrm{C}, 2.3(1.4) \mathrm{mg} / \mathrm{d}$ for azooxanthellate corals at $24^{\circ} \mathrm{C}, 2.3(0.9) \mathrm{mg} / \mathrm{d}$ for zooxanthellate corals at $16^{\circ} \mathrm{C} 2.3(0.8) \mathrm{mg} / \mathrm{d}$ for azooxanthellate corals at $16^{\circ} \mathrm{C}$. Overall treatment growth rates were $5.5(2.5) \mathrm{mg} / \mathrm{d}$ for zooxanthellate corals at $24^{\circ} \mathrm{C}, 3.7(2.9) \mathrm{mg} / \mathrm{d}$ for azooxanthellate corals at $24^{\circ} \mathrm{C}, 3.1(1.1)$ $\mathrm{mg} / \mathrm{d}$ for zooxanthellate corals at $16^{\circ} \mathrm{C} 3.6(1.5) \mathrm{mg} / \mathrm{d}$ for azooxanthellate corals at $16^{\circ} \mathrm{C}$. Increased growth rates for the treatment period relative to the pre-treatment period reflect, in-part, an increase in the surface area of the colony over the course of the experiment. $\mathrm{CO}_{2}$ and nutrient treatments had no statistically significant effect on growth rate at $16^{\circ} \mathrm{C}$ (Fig. 1). At $24^{\circ} \mathrm{C}, \mathrm{CO}_{2}$ had a significant $(\mathrm{p}<0.05)$ negative effect on growth rate for zooxanthellate corals, but not for azooxanthellate corals. Associated ANOVA tables are given in Table A-2.

In the overall data set gender was highly significant $(\mathrm{p}<0.01)$. Buoyant weight calcification rates (Fig. A-3e-f) show female corals have lower growth rates than males, and the difference between the two genders is greater at $24^{\circ} \mathrm{C}$ than at $16^{\circ} \mathrm{C}$. The difference in growth rate between the treatment phase and the initial phase was generally greater than 0 indicating increased growth rates for most corals (Fig. 1,2). In 10 instances growth rates were lower under treatment conditions relative to pre-treatment conditions, all were associated with $\mathrm{CO}_{2}$ or $\mathrm{CO}_{2}$ and nutrient treatments. At $24^{\circ} \mathrm{C}$, only females showed lower rates under treatment conditions (Fig. 2), at $16^{\circ} \mathrm{C}$ there was no consistent pattern with respect to gender.

To test for a possible interaction between gender and treatment response, buoyant weight growth rate data from zooxanthellate and azooxanthellate corals were pooled to 
provide enough replicates of each gender. Since ambient nutrient levels were substantially (2x or $5 \mathrm{x}$ pre-treatment values for $\mathrm{NO}_{3}{ }^{-}$and $\mathrm{PO}_{4}{ }^{3-}$ respectively, equivalent to $50 \%$ and $70 \%$ of the nutrient treatment values used in Chapter 3 for nitrate and phosphate respectively) elevated during the treatment period (Table 1), data from ambient and nutrient enriched treatments were pooled as well. Relative changes in growth rate at $24^{\circ} \mathrm{C}$ (Fig. 3a), were affected by $\mathrm{CO}_{2}$ and gender $(\mathrm{p}<0.01)$, as well as the interaction of $\mathrm{CO}_{2}$ and gender $(\mathrm{p}<0.05)$. At $16^{\circ} \mathrm{C}($ Fig. $3 \mathrm{~b}), \mathrm{CO}_{2}$ had a significant effect $(\mathrm{p}<0.05)$, and the parent colony was significant $(\mathrm{p}<0.01)$, but no significant gender effect was found. All colonies survived and grew for the duration of the experiment.

\section{Discussion}

Consistent with previous studies (e.g. Pilson et al., 1983), growth rates were lower at $16^{\circ} \mathrm{C}$ than at $24^{\circ} \mathrm{C}$, and at $24^{\circ} \mathrm{C}$, growth rates of zooxanthellate corals were higher than those of non zooxanthellate corals - for non- $\mathrm{CO}_{2}$ treated corals: pre-treatment, zooxanthellate colonies grew at a rate of $2.5(1.1) \mathrm{mg} / \mathrm{d}$, azooxanthellate colonies at 2.2 (1) $\mathrm{mg} / \mathrm{d}$, during the treatment phase zooxanthellate colonies grew at a rate of $3.5(1.2)$ $\mathrm{mg} / \mathrm{d}$, azooxanthellate colonies at $3.8,(1.8) \mathrm{mg} / \mathrm{d}$ at $16^{\circ} \mathrm{C}$ versus pre-treatment rates for zooxanthellate colonies of 3.1 (1) $\mathrm{mg} / \mathrm{d}$, azooxanthellate $2.7(1.5) \mathrm{mg} / \mathrm{d}$, and treatment rates for zooxanthellate colonies of $6(2) \mathrm{mg} / \mathrm{d}$, and azooxanthellate $4.6(2.6) \mathrm{mg} / \mathrm{d}$ at $24^{\circ} \mathrm{C}$.

For comparison purposes, growth rates during the treatment period are normalized to final skeletal mass for both the current study (Fig. A-13) and for the previous study 
(Chapter 3 Appendix). No treatment effect is evident; however there are consistent differences between the studies, with corals grown for Chapter 3 showing consistently lower growth rates. Increased growth rates associated with the treatment phase of the current experiment cannot account for the difference as growth rates were higher during the pre-treatment phase as well $\left(\sim 2 \mathrm{mg} \mathrm{CaCO}_{3}\right.$ added per g skeleton /day in the current study versus $<1.5$ in Chapter 3 ). Though feeding rates were not quantified for Chapter 3 , rates were likely lower than those used for the current study, so the growth rate difference may reflect differences in food availability (e.g. Houlbreque et al., 2004).

In the current study, coral calcification was impacted by elevated $\mathrm{pCO}_{2}$, however the significance of the $\mathrm{CO}_{2}$ response varied among treatments and gender. For zooxanthellate corals, an increase in ambient nutrient levels starting shortly before the initiation of treatments (Fig. A-1e) may have influenced results by supplying additional nutrients to the zooxanthellae and potentially enhancing photosynthesis. Throughout the treatment phase, ambient nutrient levels were over $50 \%$ of the desired treatment levels, thus there is no low-nutrient control for the current experiment, unlike the previous run (Chapter 3). Female corals showed a more pronounced sensitivity to $\mathrm{CO}_{2}\left(\mathrm{CO}_{2}\right.$ treated corals had relative calcification rates $86 \%$ of ambient calcification at $16^{\circ} \mathrm{C}, 56 \%$ at $24^{\circ} \mathrm{C}$ for female corals versus $82 \%$ and $97 \%$ for males) suggesting gender affects coral sensitivity to saturation state. The gender difference in $\mathrm{CO}_{2}$ sensitivity was significant only at temperatures where spawning was observed $\left(24^{\circ} \mathrm{C}\right)$, suggesting a link between spawning and calcification.

Zooxanthellate and azooxanthellate coral growth 
Significantly reduced rates of calcification in response to elevated $\mathrm{pCO}_{2}$ were found for zooxanthellate Astrangia poculata grown at $24^{\circ} \mathrm{C}$. These results are similar to those of other studies which have documented declines in calcification in response to elevated $\mathrm{pCO}_{2}$ or acidification (e.g. Langdon et al., 2000; Leclerq et al., 2002; Marubini et al., 2003; Reynaud et al., 2003; Ohde and Hossain, 2004; Renegar and Riegl 2005; Schneider and Erez, 2006; Holcomb et al. submitted). Growth rates tended to increase throughout the study, partly as a result of the increase in colony size; increases were greater for corals not exposed to $\mathrm{CO}_{2}$ (Fig. 1a, A- 3a). The increase in growth rate seen in both ambient and nutrient enriched treatments corresponds to an increase in dissolved nutrients in the source seawater (Fig. A-1e,f, A-3a). Since oxygen consumption data suggest zooxanthellate corals at $24^{\circ} \mathrm{C}$ are respiring at a greater rate than photosynthesis is occurring (Fig. A- 6a), and PAM fluorescence (Fig. A- 8), and visual inspection suggest relatively low pigment content, $\mathrm{CO}_{2}$ availability is unlikely to limit either calcification or photosynthesis. Nutrients (such as phosphate) may have limited zooxanthellae growth during much of the pre-treatment phase as suggested by the increase in coral growth rate (Fig. 1), and a slight reduction in respiration rates in the light (Fig. A-6a) coinciding with the rise in ambient nutrient levels (Fig. A- 1e,f). Thus, an increase in photosynthesis could account for the increase in calcification in zooxanthellate corals not exposed to elevated $\mathrm{pCO}_{2}$ at $24^{\circ} \mathrm{C}$. Since $\mathrm{CO}_{2}$ is unlikely to be limiting for either calcification or photosynthesis, the impact of $\mathrm{CO}_{2}$ on saturation state may dominate the calcification response. In Chapter 3, nutrients appeared to interact positively with $\mathrm{CO}_{2}$, while in the current study no such interaction was observed, this may reflect a lack of nutrient 
limitation during the treatment period of the current study and a difference in symbiont density. Though symbiont density was not measured in either study, zooxanthellate colonies appeared lighter in the current experiment, suggesting a lower symbiont density, thus $\mathrm{CO}_{2}$ and nutrient limitation are less likely to occur. The lack of a significant $\mathrm{CO}_{2}$ effect for azooxanthellate corals may indicate the negative impact of $\mathrm{CO}_{2}$ on calcification alone may be insufficient to account for the results, however calcification rates do appear lower in $\mathrm{CO}_{2}$ treated azooxanthellate corals (Fig. 1b).

Given the high ambient nutrient levels (Table 1), an effect from the nutrient treatment is unlikely though growth rates do appear slightly higher in nutrient treated azooxanthellate corals for unknown reasons (Fig. 1). At lower temperatures, calcification rates are lower (Fig. 1), and thus the magnitude of the $\mathrm{pCO}_{2}$ effect may be correspondingly reduced. In azooxanthellate corals where $\mathrm{CO}_{2}$ availability should not be limiting and the only major process taking up $\mathrm{CO}_{2}$ should be calcification, saturation state would be expected to strongly influence calcification. However the decline in calcification rates is not significant (Fig. 1). This could suggest that calcification is not strongly dependent upon the saturation state of the external environment, or may reflect that the sample size was not large enough to detect a significant effect. For zooxanthellate corals grown at $24^{\circ} \mathrm{C}$, the probability of failing to detect as significant $\mathrm{CO}_{2}$ difference if one existed was $30 \%$, for all other treatments the probability was greater than $50 \%$ (power calculations from Zar 1984). Thus, $\mathrm{CO}_{2}$ treatment may have reduced calcification rates in all treatments, but the sample size was too small for this reduction to be detected as significant in most cases. 


\section{Gender effect}

The spawning patterns observed here are consistent with the observations of Szmant et al. (1980); all polyps of a given colony were of the same gender, and reproduction appeared to be linked to temperature. Szmant et al. (1980) observed mature sperm more frequently than mature eggs, consistent with more frequent observations of sperm release in the current study. Growth rates of female corals were lower than males regardless of temperature or symbiont status, however differences were more pronounced (up to $76 \%$ lower) at $24^{\circ} \mathrm{C}$ (growth rates $\left(\mathrm{mg} / \mathrm{d}\right.$ ) for non- $\mathrm{CO}_{2}$ treated corals (nutrient treatments pooled): $16^{\circ} \mathrm{C}$ pre-treatment phase: female azooxanthallate corals $1.8(0.5)$, female zooxanthellate $1.8(0.2)$, male azooxanthellate $2.7(1.3)$, male zooxanthellate 2.7 (1.2), treatment phase: female azooxanthellate 2.6 (1.1), female zooxanthellate $3.0(0.2)$, male azooxanthellate 5.0 (1.6), male zooxanthellate $3.7(1.4) ; 24^{\circ} \mathrm{C}$ pre-treatment phase: female azooxanthellate $1.2(0.3)$, female zooxanthellate $1.9(0.1)$, male azooxanthellate 3.7 (1.0), male zooxanthellate 3.5 (1.0), treatment phase female azooxanthellate 1.7 (0.8), female zooxanthellate $3.2(0.4)$, male azooxanthellate $6.5(1)$, male zooxanthellate 7 (2)) (Fig. A-3). Such differences suggest coral gender may be a significant and unexplored factor in determining calcification rates. The apparent gender difference may be a product of the small sample size ( 8 corals total, 5 male, 3 female). However, across a wide range of treatments, all fragments consistently show similar patterns based on the gender of the source colony. Calcification rate responses to changes in $\mathrm{pCO}_{2}$ differ between genders (Fig 2,3), with male colonies appearing much less sensitive to elevated $\mathrm{pCO}_{2}$ at $24^{\circ} \mathrm{C}$, the temperature at which spawning was observed. Why gender (and 
spawning) appears related to calcification rate is unknown, but it may reflect a greater energy investment in the production of eggs relative to sperm. A greater gender based difference in growth rate at $24^{\circ} \mathrm{C}$ appears consistent with resource allocation for reproduction leading to reduced calcification. Why there is an apparent difference at $16^{\circ} \mathrm{C}$ is not clear, but there may still be a gametogenic cycle which either is not completed, or occurs at a slower rate so spawning is less frequent and thus was not observed.

Respiration rates tended to be lower in female corals (Fig. A-6), which may reflect a higher percentage of organic materials being incorporated into gametes rather than being respired to provide energy for calcification. Exposure of corals to exogenous estrogens has been found to reduce skeletal growth and increase tissue growth (Tarrant et al. 2004), thus skeletal growth may be under hormonal regulation. If egg production results in a significant reduction in energy available for other processes, female growth and reproduction could be more vulnerable to environmental variables, consistent with observations of an increase in male:female ratios on polluted reefs (Harrison and Wallace 1990). If gender and gender based calcification differences are stable over seasons, female corals would be expected to take longer to reach the same colony size as males. In work with Porites astreoides, Chornesky and Peters (1987) found reproduction of female colonies to be delayed till the coral reached a larger colony size than hermaphroditic colonies suggesting that female corals may wait to reach a certain size before devoting energy to reproduction. 


\section{Summary}

Consistent with previous results, growth rates were lower for zooxanthellate corals grown under elevated $\mathrm{pCO}_{2}$ conditions relative to corals under ambient conditions. However, only zooxanthellate corals grown at $24^{\circ} \mathrm{C}$ were significantly affected, azooxanthellate corals showed an apparent decline in calcification rate in response to the decline in saturation state. Corals spawning as males exhibited higher growth rates than those spawning as females, suggesting gender has a significant impact on calcification rate. Under elevated $\mathrm{CO}_{2}$, calcification rates were reduced to a greater degree in spawning female corals than in males. If, for gonochoric coral species, there are pronounced gender differences in calcification rates, this may be an as yet unexplored factor influencing skeletal density banding and trace element signals, and coral sensitivity to environmental variables such as $\mathrm{CO}_{2}$. 


\section{Acknowledgments}

This work benefited from the assistance of Dave Wellwood who analyzed salinity, Rebecca Belastock for DIC, Paul Henderson who analyzed nutrients, Dan Schrag and Greg Eischeid for $\mathrm{d}^{18} \mathrm{O}$ measurements, Anne Cohen, Dan McCorkle, Scott Gallager and Ann Tarrant who provided helpful suggestions on running the experiment, Jason Smith who helped calibrate temperature probes, Amber York, Fred Keller and Kevin Thompson who help to keep the seawater supply running, Michael Brosnahan, Nan Trowbridge, and Terry Rioux for diving assistance. Funding for this work was provided by the Ocean Life

Institute, NSF OCE-0648157, and an International Society for Reef Studies / Ocean Conservancy Fellowship. This material is based upon work supported under a National Science Foundation Graduate Research Fellowship. 


\section{References}

Anthony, K.R.N., Kline, D.I., Diaz-Pulido, G., Dove, S., Hoegh-Guldberg, O., 2008. Ocean acidification causes bleaching and productivity loss in coral reef builders. Proc Natl Acad Sci USA 105, 17442-17446.

Benson, B.B. and Krause, D. Jr. 1984. The concentration and isotopic fractionation of oxygen dissolved in freshwater and seawater in equilibrium with the atmosphere. Limnol. Oceanogr. 29: 620-632.

Boschma, H. 1925. On the feeding reactions and digestion in the coral polyp Astrangia danae, with notes on its symbiosis with zooxanthellae. Biological Bulletin, The 49: 407-439.

Chornesky, E.A. and E.C. Peters 1987. Sexual reproduction and colony growth in the scleractinian coral Porites astreoides. Biol. Bull 172: 161-177

Delvalls, T. A. and A. G. Dickson 1998. The pH of buffers based on 2-amino-2hydroxymethyl-1,3-propanediol ('tris') in synthetic sea water. Deep-Sea Research 45: 1541-1554.

Dickson, A.G. 1990. Standard potential of the reaction: $\mathrm{AgCl}(\mathrm{s})+1 / 2 \mathrm{H} 2(\mathrm{~g})=\mathrm{Ag}(\mathrm{s})+$ $\mathrm{HCl}(\mathrm{aq})$, and the standard acidity constant of the ion HSO4- in synthetic seawater from 273.15 to 318.15 K. J. Chem. Thermodynamics 22:113-127.

Dickson, A. G., and F. J. Millero. 1987. A comparison of the equilibrium constants for the dissociation of carbonic acid in seawater media. Deep-Sea Research 34:17331743.

DOE, eds, Dickson, A.G., Goyet, C., 1994. Handbook of Methods for the Analysis of the Various Parameters of the Carbon Dioxide System in Sea Water version 2. ORNL/CDIAC-74.

Fofonoff, N. 1985. Physical Properties of Seawater A New Salinity Scale and Equation of State for Seawater. J. Geophys. Res. 90: 3332-3342.

Garcia, H. E. and L. I. Gordon 1992. Oxygen solubility in seawater: Better fitting equations. Limnol. Oceanogr. 37: 1307-1312.

Harrison, P. L. and C. C. Wallace 1990. Reproduction, dispersal and recruitment of scleractinian corals. in Coral Reefs ed Z Dubinsky . 133-207.

Holcomb, M., McCorkle, D.C., Cohen, A.L. (submitted). Long-term effects of nutrient and $\mathrm{CO} 2$ enrichment on the temperate coral Astrangia poculata (Ellis and Solander, 1786) JEMBE

Houlbreque, F., Tambutte, E., Allemand, D., Ferrier-Pages, C., 2004. Interactions between zooplankton feeding, photosynthesis and skeletal growth in the scleractinian coral Stylophora pistillata. J. Exp. Biol. 207, 1461-1469.

Jacques TG, Marshall N, Pilson MEQ (1983) Experimental ecology of the temperate scleractinian coral Astrangia danae II. Effect of temperature, light intensity and symbiosis with zooxanthellae on metabolic rate and calcification. Marine Biol. 76: $135-148$

Jury CP, Whitehead RF, Szmant AM (accepted) Effects of variations in carbonate chemistry on the calcification rates of Madracis mirabilis (Duchassaing 1861): 
Bicarbonate concentrations best predict calcification rates. Global Change Biology

Kleypas, J. A., R. W. Buddemeier, et al. 1999. Geochemical consequences of increased atmospheric carbon dioxide on coral reefs. Science 284: 118-120.

Langdon, C., Atkinson, M.J., 2005. Effect of elevated pCO2 on photosynthesis and calcification of corals and interactions with seasonal change in temperature/irradiance and nutrient enrichment. J. Geophys. Res. 110.

Langdon, C., Takahashi, T., Sweeney, C., Chipman, D., Goddard, J., Marubini, F., Aceves, H., Barnett, H., Atkinson, M.J., 2000. Effect of calcium carbonate saturation state on the calcification rate of an experimental coral reef. Global Biogeochem Cycles 14, 639-654.

Leclercq, N., Gattuso, J.-P., Jaubert, J., 2002. Primary production, respiration,and calcification of a coral reef mesocosm under increased $\mathrm{CO} 2$ partial pressure. Limnol. Oceanography 47, 558-564.

Marubini, F., Davies, P.S., 1996. Nitrate increases zooxanthellae population density and reduces skeletogenesis in corals. Marine Biol. 127, 319-328.

Marubini, F., Atkinson, M.J., 1999. Effects of lowered $\mathrm{pH}$ and elevated nitrate on coral calcification. Mar. Ecol. Prog. Ser. 188, 117-121.

Marubini, F., Ferrier-Pages, C., Cuif, J.-P., 2003. Suppression of skeletal growth in scleractinian corals by decreasing ambient carbonate-ion concentration: a crossfamily comparison. Proc. R. Soc. Lond. B 270, 179-184.

Mehrbach, C., Culberson, C.H., Hawley, J.E., Pytkowicz, R.M., 1973. Measurement of the apparent dissociation constants of carbonic acid in seawater at atmospheric pressure. Limnol. Oceanography 18, 897-907.

Ohde, S., Hossain, M.M.M., 2004. Effect of CaCO3 (aragonite) saturation state of seawater on calcification of Porites coral. Geochem J 38, 613-621.

Pierrot, D. E. Lewis, and D. W. R. Wallace. 2006. MS Excel Program Developed for CO2 System Calculations. ORNL/CDIAC-105a. Carbon Dioxide Information Analysis Center, Oak Ridge National Laboratory, U.S. Department of Energy, Oak Ridge, Tennessee.

Renegar, D.A., Riegl, B.M., 2005. Effect of nutrient enrichment and elevated CO2 partial pressure on growth rate of Atlantic scleractinian coral Acropora cervicornis. Mar. Ecol. Prog. Ser. 293, 69-76.

Reynaud, S., Leclerq, N., Romaine-Lioud, S., Ferrier-Pages, C., Jaubert, J., Gattuso, J.-P., 2003. Interacting effects of $\mathrm{CO} 2$ partial pressure and temperature on photosynthesis and calcification in a scleractinian coral. Global Change Biol. 9, 1660-1668.

Schneider, K., Erez, J., 2006. The effect of carbonate chemistry on calcification and photosynthesis in the hermatypic coral Acropora eurystoma. Limnol. Oceanogr. 51, 1284-1293.

Szmant-Froelich, A., P. Yevich, et al. (1980). Gametogenesis and early development of the temperate coral Astrangia danae (Anthozoa: scleractinia). Biological Bulletin, The 158: 257-269. 
Tarrant, A. M., M. J. Atkinson, et al. (2004). Effects of steroidal estrogens on coral growth and reproduction. Mar. Ecol. Prog. Ser. 269: 121-129.

Zhang, H. and R. H. Byrne (1996). Spectrophotometric $\mathrm{pH}$ measurements of surface seawater at in-situ conditions: absorbance and protonation behavior of thymol blue. Mar. Chem. 52: 17-25. 
Table 1. Water chemistry during initial and treatment phases. Average alkalinity $(\mu \mathrm{mol} / \mathrm{kg}), \mathrm{pH}$ (total scale, value at experimental temperature), saturation state $(\Omega), \mathrm{CO}_{3}{ }^{2-}$ $(\mu \mathrm{mol} / \mathrm{kg}), \mathrm{HCO}_{3}{ }^{-}(\mu \mathrm{mol} / \mathrm{kg}), \mathrm{pCO}_{2}(\mu \mathrm{atm})$, salinity, $\mathrm{NH}_{4}{ }^{+}, \mathrm{H}_{2} \mathrm{SiO}_{4}, \mathrm{PO}_{4}{ }^{3-}$, and $\mathrm{NO}_{3}{ }^{-}$ $(\mu \mathrm{M})$. Measured alkalinity, temperature, salinity, nutrient, and $\mathrm{pH}_{\mathrm{T}}$ values were used to calculate saturation state and carbon speciation using a Matlab implementation of CO2SYS which incorporated the contribution of ammonia. Constants of Mehrbach et al. (1973) as refit by Dickson and Millero (1987) were used for carbonate speciation, Dickson (1990) for sulfate. 


\begin{tabular}{|c|c|c|c|c|c|c|c|c|c|c|}
\hline Initial & Alk & $\mathrm{pH}_{\mathrm{T}}$ & $\Omega_{\text {Arag }}$ & $\mathrm{CO}_{3}{ }^{2-}$ & $\mathrm{HCO}_{3}^{-}$ & $\mathrm{pCO}_{2}$ & S & $\mathrm{NH}_{4}^{+}$ & $\mathrm{H}_{2} \mathrm{SiO}_{4}$ & $\mathrm{PO}_{4}{ }^{3-}$ \\
\hline $24^{\circ} \mathrm{C}$ ambient & $\begin{array}{l}2110 \\
(20)\end{array}$ & $\begin{array}{l}7.98 \\
(0.02)\end{array}$ & $\begin{array}{l}2.5 \\
(0.1)\end{array}$ & $\begin{array}{l}160 \\
(10)\end{array}$ & $\begin{array}{l}1730 \\
(30)\end{array}$ & $\begin{array}{l}450 \\
(30)\end{array}$ & $\begin{array}{l}32 \\
(0.4)\end{array}$ & $\begin{array}{l}1 \\
(0.3)\end{array}$ & $\begin{array}{l}3.4 \\
(2)\end{array}$ & $\begin{array}{l}0.3 \\
(0.4)\end{array}$ \\
\hline $24^{\circ} \mathrm{C}$ nutrient & $\begin{array}{l}2110 \\
(20)\end{array}$ & $\begin{array}{l}7.99 \\
(0.4)\end{array}$ & $\begin{array}{l}2.5 \\
(0.1)\end{array}$ & $\begin{array}{l}160 \\
(10)\end{array}$ & $\begin{array}{l}1720 \\
(20)\end{array}$ & $\begin{array}{l}440 \\
(40)\end{array}$ & $\begin{array}{l}32 \\
(0.4)\end{array}$ & $\begin{array}{l}0.9 \\
(0.4)\end{array}$ & $\begin{array}{l}3.5 \\
(1.7)\end{array}$ & $\begin{array}{l}0.2 \\
(0.2)\end{array}$ \\
\hline $24^{\circ} \mathrm{C} \mathrm{CO}_{2}$ & $\begin{array}{l}2120 \\
(20)\end{array}$ & $\begin{array}{l}7.97 \\
(0.04)\end{array}$ & $\begin{array}{l}2.4 \\
(0.2)\end{array}$ & $\begin{array}{l}150 \\
(10)\end{array}$ & $\begin{array}{l}1740 \\
(30)\end{array}$ & $\begin{array}{l}460 \\
(50)\end{array}$ & $\begin{array}{l}32 \\
(0.4)\end{array}$ & $\begin{array}{l}0.9 \\
(0.2)\end{array}$ & $\begin{array}{l}3.4 \\
(1.5)\end{array}$ & $\begin{array}{l}0.2 \\
(0.1)\end{array}$ \\
\hline $\begin{array}{l}24^{\circ} \mathrm{C} \text { nutrient } \\
\& \mathrm{CO}_{2}\end{array}$ & $\begin{array}{l}2120 \\
(20)\end{array}$ & $\begin{array}{l}7.97 \\
(0.05)\end{array}$ & $\begin{array}{l}2.4 \\
(0.2)\end{array}$ & $\begin{array}{l}150 \\
(10)\end{array}$ & $\begin{array}{l}1740 \\
(20)\end{array}$ & $\begin{array}{l}460 \\
(60)\end{array}$ & $\begin{array}{l}32 \\
(0.4)\end{array}$ & $\begin{array}{l}0.7 \\
(0.3)\end{array}$ & $\begin{array}{l}3.3 \\
(1.3)\end{array}$ & $\begin{array}{l}0.2 \\
(0.1)\end{array}$ \\
\hline $16^{\circ} \mathrm{C}$ ambient & $\begin{array}{l}2120 \\
(20)\end{array}$ & $\begin{array}{l}8.02 \\
(0.03)\end{array}$ & $\begin{array}{l}2.1 \\
(0.1)\end{array}$ & $\begin{array}{l}130 \\
(10)\end{array}$ & $\begin{array}{l}1790 \\
(20)\end{array}$ & $\begin{array}{l}400 \\
(30)\end{array}$ & $\begin{array}{l}31 \\
(0.3)\end{array}$ & $\begin{array}{l}1.0 \\
(0.4)\end{array}$ & $\begin{array}{l}3.2 \\
(1)\end{array}$ & $\begin{array}{l}0.2 \\
(0.1)\end{array}$ \\
\hline $16^{\circ} \mathrm{C}$ nutrient & $\begin{array}{l}2110 \\
(20)\end{array}$ & $\begin{array}{l}8.00 \\
(0.03)\end{array}$ & $\begin{array}{l}2.0 \\
(0.1)\end{array}$ & $\begin{array}{l}130 \\
(10)\end{array}$ & $\begin{array}{l}1800 \\
(20)\end{array}$ & $\begin{array}{l}430 \\
(40)\end{array}$ & $\begin{array}{l}31 \\
(0.4)\end{array}$ & $\begin{array}{l}1.0 \\
(0.4)\end{array}$ & $\begin{array}{l}3.7 \\
(1.5)\end{array}$ & $\begin{array}{l}0.3 \\
(0.2)\end{array}$ \\
\hline $16^{\circ} \mathrm{C} \mathrm{CO}_{2}$ & $\begin{array}{l}2120 \\
(20)\end{array}$ & $\begin{array}{l}8.00 \\
(0.05)\end{array}$ & $\begin{array}{l}2.0 \\
(0.2)\end{array}$ & $\begin{array}{l}130 \\
(10)\end{array}$ & $\begin{array}{l}1790 \\
(20)\end{array}$ & $\begin{array}{l}430 \\
(70)\end{array}$ & $\begin{array}{l}31 \\
(0.4)\end{array}$ & $\begin{array}{l}0.8 \\
(0.2)\end{array}$ & $\begin{array}{l}3.4 \\
(1.7)\end{array}$ & $\begin{array}{l}0.2 \\
(0.1)\end{array}$ \\
\hline $\begin{array}{l}16^{\circ} \mathrm{C} \text { nutrient } \\
\& \mathrm{CO}_{2} \\
\text { Treatment }\end{array}$ & $\begin{array}{l}2120 \\
(20)\end{array}$ & $\begin{array}{l}7.98 \\
(0.05)\end{array}$ & $\begin{array}{l}2.0 \\
(0.2)\end{array}$ & $\begin{array}{l}130 \\
(10)\end{array}$ & $\begin{array}{l}1800 \\
(20)\end{array}$ & $\begin{array}{l}450 \\
(70)\end{array}$ & $\begin{array}{l}31 \\
(0.5)\end{array}$ & $\begin{array}{l}1.1 \\
(0.3)\end{array}$ & $\begin{array}{l}3.3 \\
(1.7)\end{array}$ & $\begin{array}{l}0.3 \\
(0.2)\end{array}$ \\
\hline $24^{\circ} \mathrm{C}$ ambient & $\begin{array}{l}2040 \\
(20)\end{array}$ & $\begin{array}{l}8.00 \\
(0.04)\end{array}$ & $\begin{array}{l}2.5 \\
(0.2)\end{array}$ & $\begin{array}{l}160 \\
(10)\end{array}$ & $\begin{array}{l}1650 \\
(40)\end{array}$ & $\begin{array}{l}410 \\
(40)\end{array}$ & $\begin{array}{l}31 \\
(0.6)\end{array}$ & $\begin{array}{l}1 \\
(0.4)\end{array}$ & $\begin{array}{l}5 \\
(1)\end{array}$ & $\begin{array}{l}0.6 \\
(0.1)\end{array}$ \\
\hline $24^{\circ} \mathrm{C}$ nutrient & $\begin{array}{l}2040 \\
(20)\end{array}$ & $\begin{array}{l}8.00 \\
(0.03)\end{array}$ & $\begin{array}{l}2.5 \\
(0.1)\end{array}$ & $\begin{array}{l}160 \\
(10)\end{array}$ & $\begin{array}{l}1650 \\
(30)\end{array}$ & $\begin{array}{l}410 \\
(30)\end{array}$ & $\begin{array}{l}31 \\
(0.6)\end{array}$ & $\begin{array}{l}1.1 \\
(0.3)\end{array}$ & $\begin{array}{l}4.9 \\
(0.8)\end{array}$ & $\begin{array}{l}0.9 \\
(0.2)\end{array}$ \\
\hline $24^{\circ} \mathrm{C} \mathrm{CO}_{2}$ & $\begin{array}{l}2050 \\
(20)\end{array}$ & $\begin{array}{l}7.81 \\
(0.03)\end{array}$ & $\begin{array}{l}1.8 \\
(0.1)\end{array}$ & $\begin{array}{l}110 \\
(10)\end{array}$ & $\begin{array}{l}1780 \\
(30)\end{array}$ & $\begin{array}{l}690 \\
(60)\end{array}$ & $\begin{array}{l}31 \\
(0.6)\end{array}$ & $\begin{array}{l}1.1 \\
(0.2)\end{array}$ & $\begin{array}{l}5.4 \\
(0.8)\end{array}$ & $\begin{array}{l}0.6 \\
(0.2)\end{array}$ \\
\hline $\begin{array}{l}24^{\circ} \mathrm{C} \text { nutrient } \\
\& \mathrm{CO}_{2}\end{array}$ & $\begin{array}{l}2050 \\
(30)\end{array}$ & $\begin{array}{l}7.78 \\
(0.04)\end{array}$ & $\begin{array}{l}1.7 \\
(0.2)\end{array}$ & $\begin{array}{l}100 \\
(10)\end{array}$ & $\begin{array}{l}1790 \\
(30)\end{array}$ & $\begin{array}{l}750 \\
(80)\end{array}$ & $\begin{array}{l}31 \\
(0.6)\end{array}$ & $\begin{array}{l}0.8 \\
(0.2)\end{array}$ & $\begin{array}{l}5.5 \\
(0.5)\end{array}$ & $\begin{array}{l}0.9 \\
(0.1)\end{array}$ \\
\hline $16^{\circ} \mathrm{C}$ ambient & $\begin{array}{l}2040 \\
(30)\end{array}$ & $\begin{array}{l}8.03 \\
(0.02)\end{array}$ & $\begin{array}{l}2.1 \\
(0.1)\end{array}$ & $\begin{array}{l}130 \\
(10)\end{array}$ & $\begin{array}{l}1710 \\
(30)\end{array}$ & $\begin{array}{l}380 \\
(30)\end{array}$ & $\begin{array}{l}30.7 \\
(0.1)\end{array}$ & $\begin{array}{l}1.1 \\
(0.2)\end{array}$ & $\begin{array}{l}3.7 \\
(1)\end{array}$ & $\begin{array}{l}0.6 \\
(0.2)\end{array}$ \\
\hline $16^{\circ} \mathrm{C}$ nutrient & $\begin{array}{l}2010 \\
(40)\end{array}$ & $\begin{array}{l}8.00 \\
(0.03)\end{array}$ & $\begin{array}{l}1.9 \\
(0.2)\end{array}$ & $\begin{array}{l}120 \\
(10)\end{array}$ & $\begin{array}{l}1700 \\
(30)\end{array}$ & $\begin{array}{l}410 \\
(40)\end{array}$ & $\begin{array}{l}30.7 \\
(0.2)\end{array}$ & $\begin{array}{l}3 \\
(2)\end{array}$ & $\begin{array}{l}2.6 \\
(0.7)\end{array}$ & $\begin{array}{l}1.4 \\
(0.3)\end{array}$ \\
\hline $16^{\circ} \mathrm{C} \mathrm{CO}_{2}$ & $\begin{array}{l}2040 \\
(30)\end{array}$ & $\begin{array}{l}7.76 \\
(0.03)\end{array}$ & $\begin{array}{l}1.2 \\
(0.1)\end{array}$ & $\begin{array}{l}80 \\
(6)\end{array}$ & $\begin{array}{l}1850 \\
(30)\end{array}$ & $\begin{array}{l}770 \\
(60)\end{array}$ & $\begin{array}{l}30.7 \\
(0.2)\end{array}$ & $\begin{array}{l}1.2 \\
(0.4)\end{array}$ & $\begin{array}{l}4.3 \\
(1.7)\end{array}$ & $\begin{array}{l}0.6 \\
(0.2)\end{array}$ \\
\hline $\begin{array}{l}16^{\circ} \mathrm{C} \text { nutrient } \\
\& \mathrm{CO}_{2}\end{array}$ & $\begin{array}{l}2030 \\
(30)\end{array}$ & $\begin{array}{l}7.74 \\
(0.04)\end{array}$ & $\begin{array}{l}1.1 \\
(0.1)\end{array}$ & $\begin{array}{l}72 \\
(5)\end{array}$ & $\begin{array}{l}1850 \\
(30)\end{array}$ & $\begin{array}{l}820 \\
(70)\end{array}$ & $\begin{array}{l}30.7 \\
(0.2)\end{array}$ & $\begin{array}{l}1.9 \\
(0.4)\end{array}$ & $\begin{array}{l}3.6 \\
(0.7)\end{array}$ & $\begin{array}{l}1.2 \\
(0.2)\end{array}$ \\
\hline
\end{tabular}



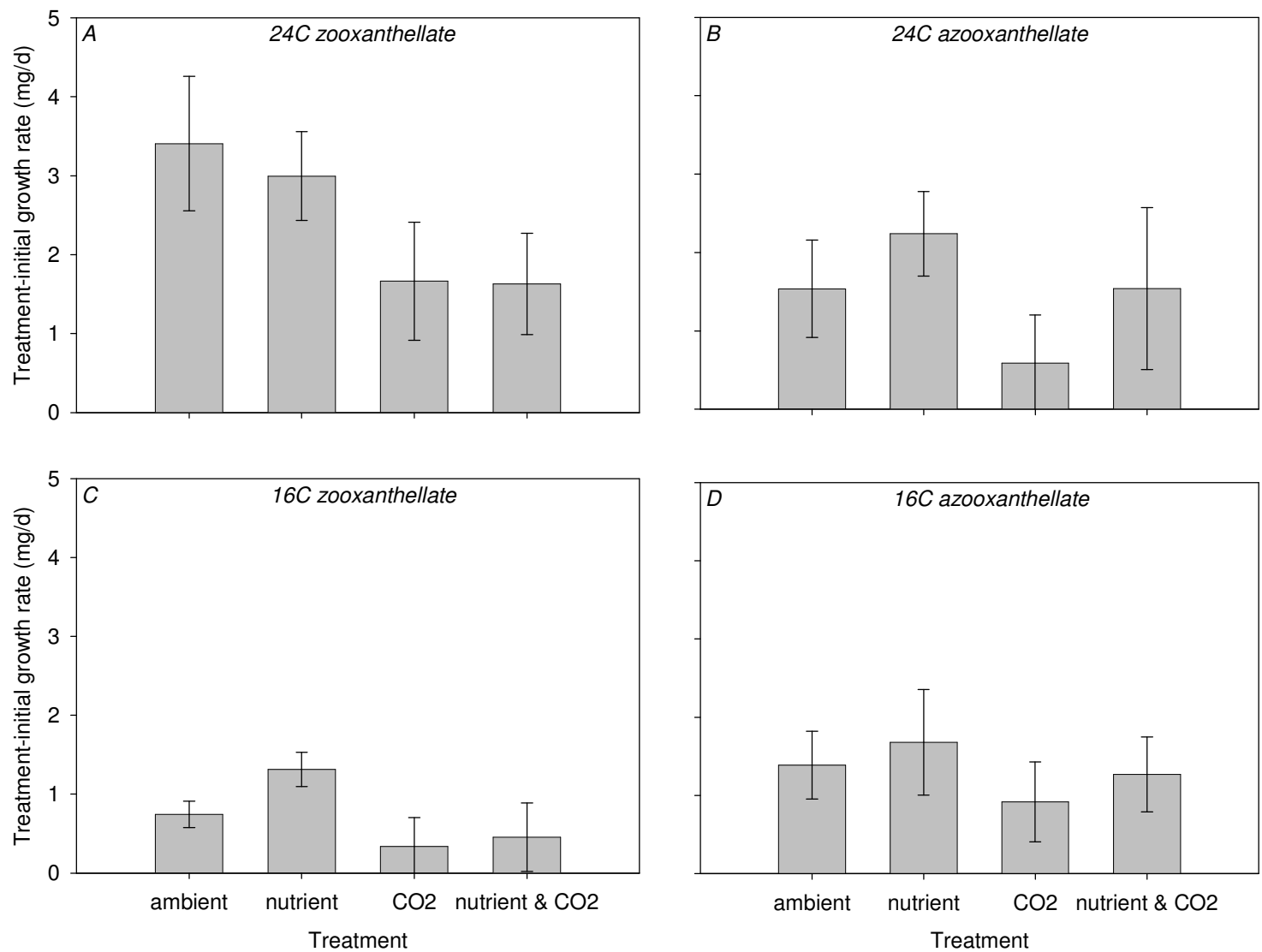

Figure 1. Calcification rate differences between initial and treatment phases as estimated from buoyant weights plotted versus treatment. A. Zooxanthellate corals grown at $24^{\circ} \mathrm{C}$. B. Azooxanthellate corals grown at $24^{\circ} \mathrm{C}$. C. Zooxanthellate corals grown at $16^{\circ} \mathrm{C} . \mathrm{D}$. Azooxanthellate corals grown at $16^{\circ} \mathrm{C}$. Bars represent average values, error bars are standard error. 

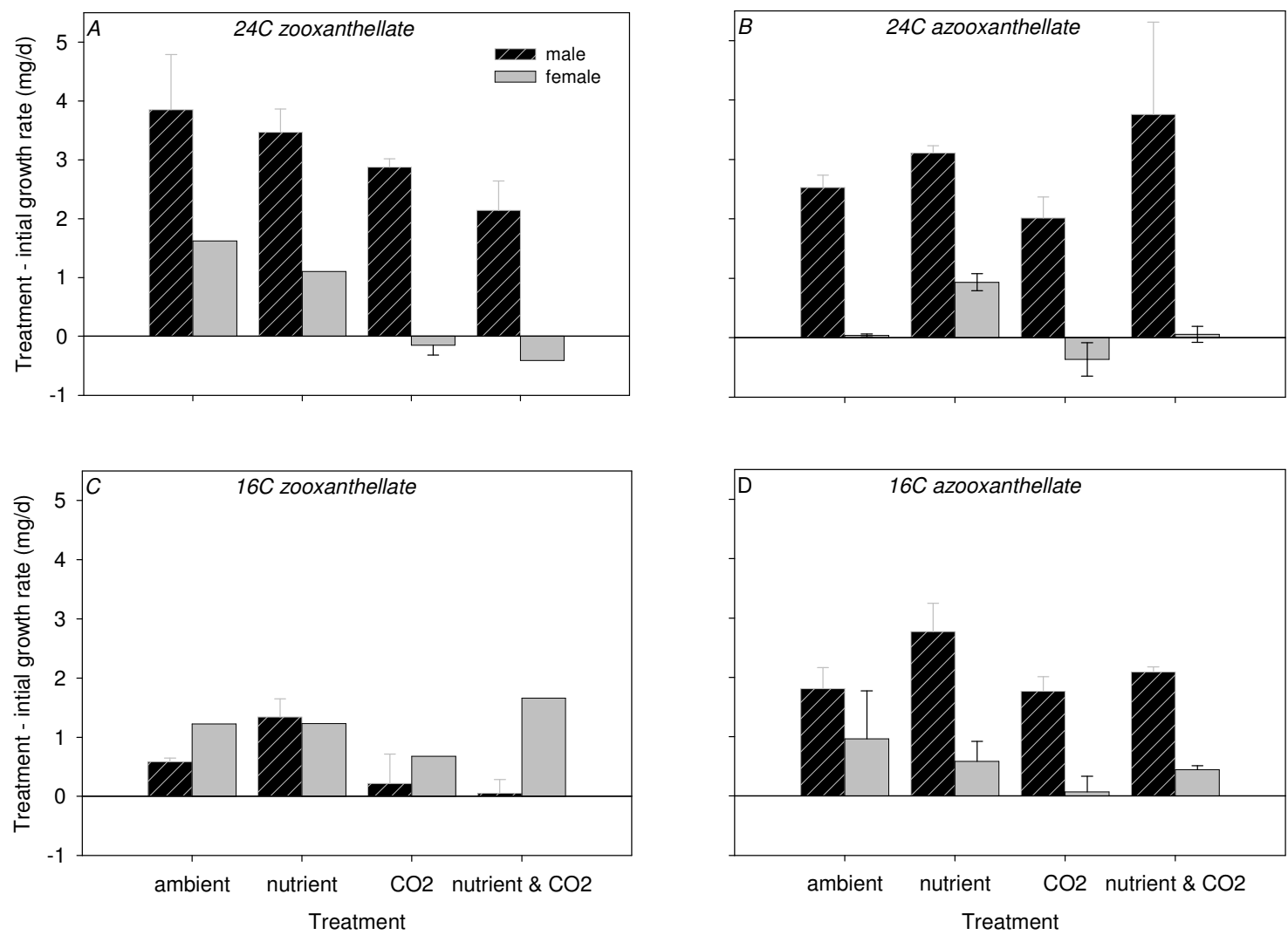

Figure 2. Calcification rate differences between initial and treatment phases as estimated from buoyant weights plotted versus treatment separated gender. Males are represented by black bars with diagonal lines, females are represented by grey bars. A. Zooxanthellate corals grown at $24^{\circ} \mathrm{C}$. B. Azooxanthellate corals grown at $24^{\circ} \mathrm{C}$. C. Zooxanthellate corals grown at $16^{\circ} \mathrm{C}$. D. Azooxanthellate corals grown at $16^{\circ} \mathrm{C}$. Bars represent average values, error bars are standard error. 

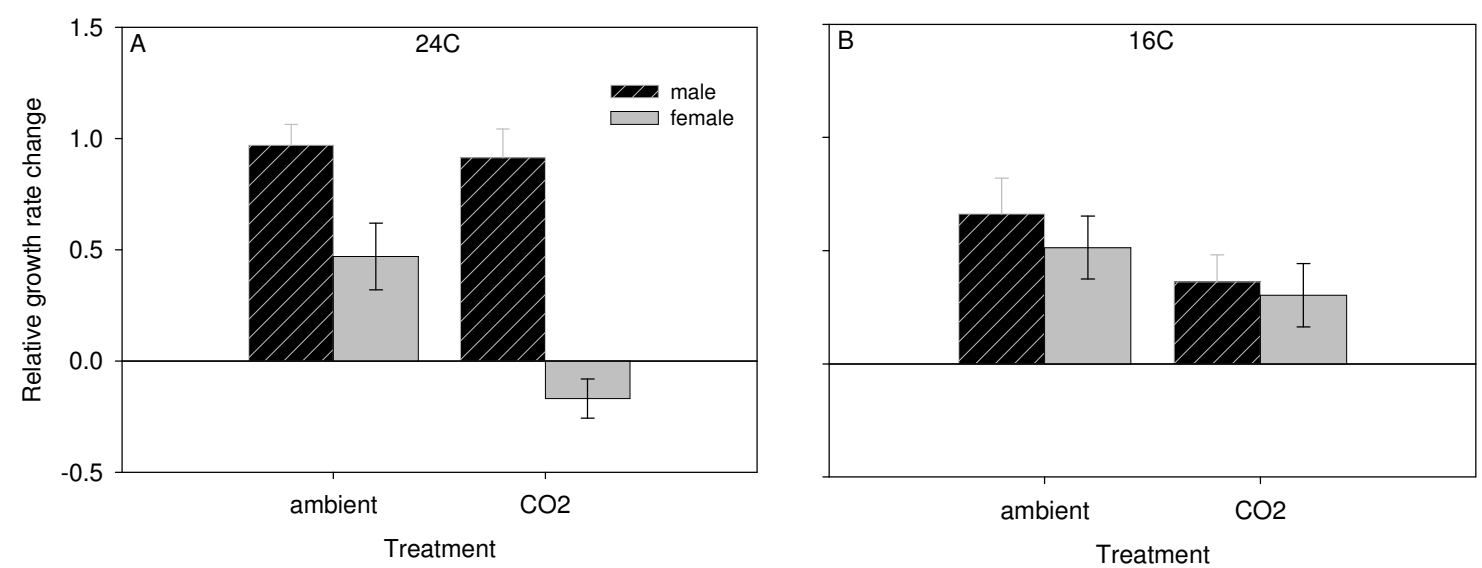

Figure 3. Relative growth rate change ((growth rate during treatment phase - growth rate during initial phase)/growth rate during initial rate) for pooled $24^{\circ} \mathrm{C}(\mathrm{A})$ and $16^{\circ} \mathrm{C}(\mathrm{B})$ data plotted by $\mathrm{CO}_{2}$ treatment and gender. Males are shown with black bars, females with grey bars. Bars represent means, error bars are standard error. A relative growth rate change of 0 means no change in growth, values below 0 indicate lower growth rates during the treatment phase, values greater than 0 indicate increased growth. 


\section{Synthesis}

In zooxanthellate corals, calcification appears to be tightly linked to photosynthesis carried out by the symbiotic zooxanthellae. The median coral calcification rate in the light is three times that in the dark, and symbiotic corals calcify at higher rates than non-symbiotic corals (e.g. Gattuso et al. 1999). When corals bleach and lose their zooxanthellae, calcification rates decline (e.g. Rodrigues and Grottoli 2006). However, the link between photosynthesis and calcification isn't direct, as factors affecting one process don't necessarily have the same effect on the other. Nutrients, particularly dissolved inorganic nitrogen (DIN) have long been known to affect rates of photosynthesis, with higher rates of carbon fixation associated with increased nutrient levels (e.g. Cook et al. 1994; Marubini and Davies 1996). Since photosynthesis is linked to calcification, it might be expected that elevated DIN levels should stimulate calcification. Since $\mathrm{HCO}_{3}{ }^{-}$is thought to be the primary source of $\mathrm{CO}_{2}$ for photosynthesis, the associated production of alkalinity alone may be sufficient to increase calcification (e.g. Al-Moghrabi et al., 1996). Oxygen production too may help to increase respiration rates and drive active transport processes. Generation of reactive oxygen species (ROS) by photosynthesis may increase membrane permeability and rates of $\mathrm{Ca}^{2+}$ transport (Sandeman 2008). Further, if the translocation of fixed carbon is increased, the coral will have both an increased supply of organic material from which to synthesize the organic matrix, as well as an energy supply to increase active transport processes. An increase in organic $\mathrm{N}$ supplied to the coral can similarly be used for organic matrix synthesis, cellular repair, fuel for active transport, or production of hydrolyzable $\mathrm{N}$ compounds for 
translocation to the calcifying environment and subsequent $\mathrm{NH}_{3}$ production for proton transport.

However, despite the potential means for nutrients to enhance calcification, the observed effects of nutrients on calcification have been mixed, with some studies showing positive calcification responses to elevated nutrients (e.g. Bongiorni et al., 2003) and others showing negative responses (e.g. Marubini and Davies 1996). Such a range of responses suggests that factors other than the photosynthetic rate alone are important for determining calcification rates. The mechanism(s) by which nutrients affect calcification are not known, however several hypothesis exist. Decreases in calcification associated with elevated nutrients may be due to: a decreased need for protons for use in nutrient acquisition, competition between photosynthesis and calcification for a limited carbon pool, the distribution of photosynthesis may shift more to the surface of the coral due to increased zooxanthellae density limiting light availability to underlying layers and thus the site of proton removal is further from the calcification site, increased use of external nutrient sources leading to reduced ammonia recycling, or reduced translocation of photosynthate from symbiont to host. Elevated calcification rates may be linked to: an increased drawdown of $\mathrm{CO}_{2}$ increasing saturation state, increased translocation of photosynthate from symbiont to host, alkalinity production, greater availability of ammonia to calcification, or increased oxygen availability. Studies combining nutrient and carbonate chemistry manipulation have found that the calcification response to nutrients depends on the $\mathrm{pCO}_{2}$ level (e.g. Chapter 3). An interaction between nutrient chemistry and carbonate chemistry suggests that carbon limitation may explain negative 
calcification responses to elevated nutrient conditions. Carbon limitation could affect calcification directly by limiting the DIC supply for calcification, or, calcification may be indirectly affected by carbon limitation of photosynthesis which could reduce the translocation of photosynthate, change the carbon species used for photosynthesis, alter the distribution of carbon uptake, or reduce ammonia uptake.

Saturation state variations within the calcifying environment of the coral may explain both growth rates and patterns of crystals within the coral skeleton (Chapter 1). Thus, any mechanism to explain how changes in nutrients affect calcification must be capable of generating changes in the saturation state of the calcifying environment. The timing of saturation state variations within the calcifying environment of the coral (Chapter 2) points to competition between photosynthesis and calcification limiting the supply of $\mathrm{CO}_{2}$ for calcification on a daily cycle. Thus, DIC availability is likely to be partly responsible for the range of responses to nutrients. If reducing $\mathrm{pCO}_{2}$ is the primary means by which zooxanthellate corals elevate saturation state, and they commonly reduce $\mathrm{pCO}_{2}$ to levels at which DIC limits calcification (Chapter 2), elevated $\mathrm{pCO}_{2}$ (external) could increase internal $\mathrm{pCO}_{2}$, leading to higher saturation state and calcification rate. Though elevated $\mathrm{pCO}_{2}$ could increase daytime (DIC limited) calcification, it may reduce calcification during periods in which $\mathrm{CO}_{2}$ is not limiting. If external $\mathrm{pCO}_{2}$ is consistently elevated (such as is the case with rising atmospheric $\mathrm{CO}_{2}$ ), the net effect will be the integral of elevated daytime calcification rates and reduced nighttime calcification rates, which may be either net positive or net negative. Since the majority of studies show external saturation state to be correlated with calcification rate (Chapter 3), the increase 
in calcification associated with relief of $\mathrm{CO}_{2}$ limitation generally makes a smaller contribution than the reduction in calcification occurring during the rest of the day. The few exceptions, in which calcification rates showed a modest increase in response to elevated $\mathrm{pCO}_{2}$, show that there are conditions under which the relief of $\mathrm{CO}_{2}$ limitation can be the dominate term, but in all cases, the effect is small. Thus, elevated $\mathrm{CO}_{2}$ is expected to negatively affect calcification, with a few exceptions which will be linked to photosynthesis limiting $\mathrm{CO}_{2}$ availability.

To increase the contribution of $\mathrm{CO}_{2}$ limitation, and thus increase the region in which elevated $\mathrm{CO}_{2}$ could increase calcification, photosynthesis could be increased. Increased nutrient levels, light levels, and temperature can all lead to increased photosynthesis, and potentially enhance daytime $\mathrm{CO}_{2}$ limitation. However, if respiration rates also increase (such as might be associated with increased zooxanthellae populations), internal $\mathrm{pCO}_{2}$ may be elevated at night, reducing nighttime saturation state and calcification rate, and leading to greater cycles in saturation state. In chapters 3 and 4, nutrient levels were manipulated in an attempt to increase photosynthesis and thus increase the daytime drawdown of $\mathrm{CO}_{2}$. In Chapter 3, there was an apparent decrease in calcification under nutrient enriched conditions, potentially reflecting enhanced photosynthetic drawdown of $\mathrm{CO}_{2}$ limiting calcification (based on Chapter 2, these corals might be expected to have more pronounced daily bands in their skeletons), however increased use of photosynthate by the zooxanthellae reducing the energy supply to the coral cannot be ruled out. Elevation of $\mathrm{CO}_{2}$ reduced calcification, consistent with the expected effect of saturation state. However, elevated $\mathrm{CO}_{2}$ combined with nutrients 
resulted in a partial recovery of calcification rates. Thus nutrients may have stimulated photosynthesis sufficiently to partly offset the elevation in $\mathrm{CO}_{2}$. However, similar patterns were not seen in Chapter 4. Several factors may have contributed to this. Experiments were carried out at lower temperatures in Chapter 4, so photosynthetic rates the contribution of photosynthesis to the coral's energy needs should be reduced (e.g. Jacques et al., 1983). Light levels were lower - though corals were generally closer to the fluorescent light bulbs for Chapter 4 experiments, the narrow tanks with black sides used for Chapter 4 reduced scattered light and light entering from high angles. Corals grown at $24^{\circ} \mathrm{C}$ for Chapter 4 were visibly lighter in coloration than those grown at $25.8^{\circ} \mathrm{C}$ for Chapter 3, so photosynthetic rates were likely lower and thus carbon limitation is less likely to occur in the Chapter 4 experiments (consistent with net oxygen consumption during the day and night as measured in Chapter 4). And, finally, nutrient levels were high throughout the treatment phase in the ambient seawater used for Chapter 4, so nutrients were unlikely to limit the growth of zooxanthellae regardless of $\mathrm{pCO}_{2}$. Such results point to the complexity of $\mathrm{CO}_{2}$ nutrient interactions. In Chapter 4, high nutrients would have been expected to increase photosynthesis, creating ideal conditions for photosynthetic limitation of calcification. However, the pigment concentration remained low, possibly as a result of the coral artificially limiting the population of zooxanthellae within its tissue. Thus the zooxanthellate colonies behaved similarly to the azooxanthellate colonies, in which in the absence of photosynthesis, elevated $\mathrm{CO}_{2}$ reduces saturation state and calcification rate. When photosynthesis occurs at a sufficient rate, $\mathrm{CO}_{2}$ could enhance photosynthesis which could in turn enhance calcification, or $\mathrm{CO}_{2}$ 
could enhance calcification directly if DIC has become limiting due to photosynthetic uptake. Factors such as nutrients, temperature, and light can influence the response to $\mathrm{CO}_{2}$ by affecting photosynthesis. However the response is not necessarily straight forward due to both physiological limitations of the zooxanthellae and due to control of the zooxanthellae population by the coral host.

Though photosynthesis is a major factor affecting calcification rates and may play an important role in determining the coral response to $\mathrm{CO}_{2}$, many corals lack zooxanthellae and thus do not carry out photosynthesis. In the absence of photosynthetic interaction with $\mathrm{CO}_{2}$, the decline in saturation state associated with elevated $\mathrm{pCO}_{2}$ might be expected to control the calcification response. However even in azooxanthellate corals of the same species, there are pronounced difference in the response to $\mathrm{CO}_{2}$ (Chapter 4). Though there were not enough replicates of just azooxanthellate corals of each gender for statistical purposes, the trend was consistent with the combined data set which shows spawning female corals to be significantly more sensitive to $\mathrm{CO}_{2}$. Spawning corals would be expected to invest more resources (energy) into reproduction than their non-spawning counter-parts. Though the energy investment in eggs relative to sperm is unknown for corals, the large size and high lipid content of eggs in many coral species suggests egg production may represent a significant energy cost (e.g., Leuzinger et al., 2003). How gamete production affects investment in other processes is unknown, however slower calcification rates for spawning female corals points to reduced energy investment in calcification. For calcification to proceed, energy must be invested in the process. The energy investment could be as small as that needed to produce an organic 
catalyst which is capable of accelerating the precipitation of aragonite directly from seawater. However, measurements of Ca concentrations (e.g. Clode and Marshall, 20002; Al-Horani et al., 2003) support active transport processes being involved in the movement of $\mathrm{Ca}^{2+}$ to the site of calcification. As $\mathrm{Ca}$ is transported in, $\mathrm{CO}_{3}{ }^{2-}$ must also be moved to the site of calcification - either through direct transport of $\mathrm{CO}_{3}{ }^{2-}$, or more likely through the removal of protons to produce $\mathrm{CO}_{3}{ }^{2-}$ from other DIC species. Though the specific pathways involved remain a subject of investigation, these transport processes require the investment of energy. Factors affecting the energy supply - food availability, photosynthate production, cellular repair, or investment in gametes may in turn affect the sensitivity of coral calcification to declines in saturation state. The greater $\mathrm{pCO}_{2}$ sensitivity of calcification in spawning females relative to spawning males suggests that males divert more energy to calcification to maintain calcification rates. Alternatively, male corals may already be adapted to higher $\mathrm{CO}_{2}$ conditions as respiration rates are likely to be high in mitochondria rich sperm relative to those in lipid rich eggs, thus internal $\mathrm{pCO}_{2}$ in male corals may be higher than that in female corals, so for a given change in external $\mathrm{pCO}_{2}$, the effect may be proportionately larger for female corals.

Under natural conditions, positive responses to elevated $\mathrm{pCO}_{2}$ are less likely than in closed aquarium systems. Rates of fluid flow tend to be relatively low in aquarium systems, thus boundary layer limitation of the $\mathrm{CO}_{2}$ supply is more likely to occur. In aquarium experiments, light levels are often kept at saturating intensities for $12 \mathrm{hr} / \mathrm{day}$, whereas in a natural environment, there is a gradual rise and decline in light, changes in cloud cover, turbidity, and other factors which reduce the period over which light is 
saturating for photosynthesis, and photoinhibition may reduce photosynthesis during high light periods. Thus the interval over which $\mathrm{CO}_{2}$ limitation of calcification could occur is correspondingly reduced, leading to a narrower window in which $\mathrm{CO}_{2}$ elevation can have a positive effect. In a natural system, though nutrients may be able to stimulate zooxanthellae growth and photosynthesis, nutrients also promote algal growth which may lead to algae out-competing corals. So though nutrients and $\mathrm{CO}_{2}$ may be able to promote calcification, the range of conditions over which this can occur in the natural environment is likely to be limited. Feeding however may provide a means by which nutrient supplies for the zooxanthellae could increase without promoting algal growth while at the same time providing energy for the coral. How feeding and $\mathrm{pCO}_{2}$ may interact remains to be tested experimentally. Based on the results presented here, higher feeding rates may be expected to increase coral resilience to declines in saturation state. Thus, understanding future changes in plankton abundance on reefs may be critical to predicting how corals in natural environments will respond to climate change. 


\section{References}

Al-Horani FA, Al-Moghrabi SM, de Beer D (2003) The mechanism of calcification and its relation to photosynthesis and respiration in the scleractinian coral Galaxea fascicularis. Marine Biol. 142: 419-426

Al-Moghrabi S, Goiran C, Allemand D, Speziale NJ, J. (1996) Inorganic carbon uptake for photosynthesis by the symbiotic coral/dinoflagellate association II.

Mechanisms for bicarbonate uptake. J. Exp. Mar. Biol. Ecol. 199: 227-248

Bongiorni L, Shafir S, Angel D, Rinkevich B (2003) Survival, growth and gonad development of two hermatypic corals subjected to in situ fish-farm nutrient enrichment. Mar. Ecol. Prog. Ser. 253: 137-144

Clode PL, Marshall AT (2002) Low temperature X-ray microanalysis of calcium in a scleractinian coral: evidence of active transport mechanisms. J. Exp. Biol. 205: 3543-3552

Cook CB, Muller-Parker G, Orlandini CD (1994) Ammonium enhancement of dark carbon fixation and nitrogen limitation in zooxanthellae symbiotic with the reef corals Madracis mirabilis and Montastrea annularis. Marine Biol. 118: 157-165

Gattuso JP, Allemand D, Frankignoulle M (1999) Photosynthesis and calcification at cellular, organismal and community levels in coral reefs: a review of interactions and control by carbonate chemistry. American Zoologist 39: 160-183

Jacques TG, Marshall N, Pilson MEQ (1983) Experimental ecology of the temperate scleractinian coral Astrangia danae II. Effect of temperature, light intensity and symbiosis with zooxanthellae on metabolic rate and calcification. Marine Biol. 76: $135-148$

Leuzinger S, Anthony KRN, Willis BL (2003) Reproductive energy investment in corals: scaling with module size. Oecologia (Berlin) 136:524-531

Marubini F, Davies PS (1996) Nitrate increases zooxanthellae population density and reduces skeletogenesis in corals. Marine Biol. 127: 319-328

Rodrigues LJ, Grottoli AG (2006) Calcification rate and the stable carbon, oxygen, and nitrogen isotopes in the skeleton, host tissue, and zooxanthellae of bleached and recovering Hawaiian corals. Geochimica et Cosmochimica Acta 70:2781-2789

Sandeman IM (2008) Fine banding in the septa of corals. Proc. 11th Int. Coral Reef Symposium 
Appendix 1 Electronic Annex for Chapter 1: Solution chemistry, pH profiles, Volumes pumped, Raman spectra, Etching comparisons, and Scale comparisons plus additional discussion and figures not included in published version

\section{Methods}

\section{Saturation state}

Saturation states $(\Omega)$ with respect to aragonite were calculated based on $\mathrm{pH}$ values (NBS scale), alkalinities and calcium concentrations measured in fluid samples taken over the course of an individual run. Two or more samples were taken over the course of a given precipitation run. Alkalinity, salinity, and cation measurements for experiments 1, 4, 5, and 6 followed methods described in Gaetani and Cohen (2006). For experiments 3 and 7 , water samples were $.45 \mu \mathrm{m}$ filtered at the time of collection. Cation measurements followed methods described in Gaetani and Cohen (2006), except Na was also measured and used to correct for sample evaporation during storage. Alkalinities were measured via titration with $0.01 \mathrm{~N} \mathrm{HCl}$ using a Metrohm Titrando 808 and 730 Sample Changer controlled by Tiamo software to perform automated gran titrations of 1 ml samples, replicate samples were run. Titrations were conducted as rapidly as possible following sample collection, high alkalinity samples were diluted with distilled water as needed. Internal seawater standards were included in each run, seawater certified reference materials (supplied by the laboratory of Andrew Dickson - SCRIPPS Institution of Oceanography) were occasionally run as well. Data for experiment 2 can be found in Gaetani and Cohen (2006), for other experiments, data are presented in Table EA-1 and Figure EA-1. The peak alkalinity value measured in each experiment should be interpreted as a minimum estimate, as precipitation occurs rapidly at these high 
supersaturation states and can lead to a reduction in measured alkalinity. Saturation state calculations were performed using an excel implementation of CO2SYS (Lewis and Wallace 1998), modified to use measured calcium concentrations. The constants of Roy et al. (1993) and Dickson (1990) were used for the carbonate system and sulfate respectively. Figures are plotted using Sigmaplot.

\section{Raman microscopy}

Raman spectra were obtained using a Kaiser Raman microprobe using a 10x or 50x objective. Excitation was by a $532 \mathrm{~nm}$ lazer operating at 4-10 $\mathrm{mW}$. Spectra were collected for 2-10 s with 5-10 accumulations. Reference raman spectra were obtained on powdered (150 $\mu \mathrm{m}$ sieved) aragonite and calcite mineral samples placed on a glass slide. Mineral samples were obtained from Ward Scientific. Synthetic precipitates were analyzed similarly. Spectra were processed using MATLAB prior to plotting with Sigmaplot.

\section{Sample names}

Some of the precipitates used in this manuscript have been used in other manuscripts, or will appear in future manuscripts, sample names corresponding to each experiment are as follows: Experiment $1=40 \mathrm{rpm}$ 7/2/06, Experiment $2=\mathrm{ASW}-7$ $\left(55^{\circ} \mathrm{C}\right)$, or ASW-8 $\left(65^{\circ} \mathrm{C}\right)$, Experiment $3=11 / 9 / 082$ cycle, Experiment 4 = PGA-4, Experiment $5=$ PGA-1, Experiment $6=$ PGA-2, Experiment $7=11 / 20 / 08$ slow . 
Table EA-1. Solution chemistry for solutions used in experiments 1, 3-7. For experiments 1,3, and 7, masses 84, 86, and 87 were measured, and the total $\mathrm{Sr}$ concentration calculated based on each isotope assuming environmental abundances. The standard error for replicate measurements is given in parenthesis. na $=$ data not available, nd $=$ not detected. 


\begin{tabular}{|c|c|c|c|c|c|c|c|c|c|c|c|c|}
\hline Experiment & time (hr) & $\mathrm{pH}$ & salinity & $\begin{array}{l}\text { alkalinity } \\
\text { (umol } / \mathrm{kg} \text { ) }\end{array}$ & \begin{tabular}{|l|}
$\mathrm{Ca}$ \\
$(\mathrm{ppm})$
\end{tabular} & $\begin{array}{l}\mathrm{Mg} \\
(\mathrm{ppm})\end{array}$ & $\begin{array}{l}84 \mathrm{Sr} \\
(\mathrm{ppm})\end{array}$ & $\begin{array}{l}86 \mathrm{Sr} \\
(\mathrm{ppm})\end{array}$ & $\begin{array}{l}88 \mathrm{Sr} \\
(\mathrm{ppm})\end{array}$ & $\begin{array}{l}\mathrm{Ba} \\
(\mathrm{ppb})\end{array}$ & $\begin{array}{l}\mathrm{Na} \\
(\%)\end{array}$ & $\Omega$ \\
\hline $\begin{array}{l}\text { Exp. } 1 \text { evaporated } \\
\text { sea water }\end{array}$ & na & na & na & na & $\begin{array}{l}742 \\
(9)\end{array}$ & $\begin{array}{l}2340 \\
(20)\end{array}$ & $\begin{array}{l}14.0 \\
(.2)\end{array}$ & $\begin{array}{l}13.9 \\
(.2)\end{array}$ & $\begin{array}{l}13.9 \\
(.1)\end{array}$ & $\begin{array}{l}15.7 \\
(.2)\end{array}$ & $\begin{array}{l}19.9 \\
(.4)\end{array}$ & na \\
\hline Exp. $1 \mathrm{Na}_{2} \mathrm{CO}_{3}$ & na & na & na & na & \begin{tabular}{|l|}
7.6 \\
$(.02)$ \\
\end{tabular} & $\begin{array}{l}13.1 \\
(.02) \\
\end{array}$ & $\begin{array}{l}0.19 \\
(.02) \\
\end{array}$ & $\begin{array}{l}0.20 \\
(.001) \\
\end{array}$ & $\begin{array}{l}0.209 \\
(.005) \\
\end{array}$ & $\begin{array}{l}1.34 \\
(.02) \\
\end{array}$ & $\begin{array}{l}1.82 \\
(.05) \\
\end{array}$ & na \\
\hline Exp. 1 sea water & na & 8.04 & 31.849 & na & $\begin{array}{l}452 \\
(.4)\end{array}$ & $\begin{array}{l}1408 \\
(2)\end{array}$ & $\begin{array}{l}8.62 \\
(.02)\end{array}$ & $\begin{array}{l}8.59 \\
(.05)\end{array}$ & $\begin{array}{l}8.5 \\
(.1)\end{array}$ & $\begin{array}{l}9.8 \\
(.1)\end{array}$ & $\begin{array}{l}11.8 \\
(.3)\end{array}$ & na \\
\hline Exp. 1 & 13.7 & 9.1 & na & 7717 & $\begin{array}{l}322 \\
(1)\end{array}$ & $\begin{array}{l}1163 \\
(3)\end{array}$ & $\begin{array}{l}6.2 \\
(.1)\end{array}$ & $\begin{array}{l}6.1 \\
(.1)\end{array}$ & $\begin{array}{l}6.1 \\
(.1)\end{array}$ & $\begin{array}{l}7.5 \\
(.3)\end{array}$ & $\begin{array}{l}9.9 \\
(.1)\end{array}$ & 33.8 \\
\hline Exp. 1 & 46.8 & 8.85 & na & 5674 & $\begin{array}{l}147 \\
(2)\end{array}$ & $\begin{array}{l}1170 \\
(10)\end{array}$ & $\begin{array}{l}5.4 \\
(.1)\end{array}$ & $\begin{array}{l}2.7 \\
(.1)\end{array}$ & $\begin{array}{l}2.7 \\
(.1)\end{array}$ & $\begin{array}{l}4.9 \\
(.3)\end{array}$ & $\begin{array}{l}10.50 \\
(.05)\end{array}$ & 9 \\
\hline Exp. 1 & 70.5 & 8.53 & 31.878 & 4602 & \begin{tabular}{|l|}
120.1 \\
$(.4)$
\end{tabular} & $\begin{array}{l}1160 \\
(10)\end{array}$ & $\begin{array}{l}4.4 \\
(.1)\end{array}$ & $\begin{array}{l}8.6 \\
(.1)\end{array}$ & $\begin{array}{l}2.22 \\
(.01)\end{array}$ & $\begin{array}{l}3.95 \\
(.03)\end{array}$ & \begin{tabular}{|l|}
10.4 \\
$(.1)$
\end{tabular} & 4 \\
\hline $\begin{array}{l}\text { Exp. } 3 \& 7 \\
\text { evaporated sea }\end{array}$ & na & na & 62.436 & $\begin{array}{l}3280 \\
(20)\end{array}$ & $\begin{array}{l}720 \\
(10)\end{array}$ & $\begin{array}{l}2350 \\
(20)\end{array}$ & $\begin{array}{l}14.2 \\
(.2)\end{array}$ & $\begin{array}{l}14.6 \\
(.2)\end{array}$ & $\begin{array}{l}14.6 \\
(.3)\end{array}$ & $\begin{array}{l}16.6 \\
(.3)\end{array}$ & $\begin{array}{l}20.3 \\
(.6)\end{array}$ & na \\
\hline Exp. $3 \& 7 \mathrm{Na}_{2} \mathrm{CO}_{3}$ & na & na & na & $\begin{array}{l}74700 \\
(400)\end{array}$ & nd & nd & nd & nd & nd & \begin{tabular}{|l}
8 \\
$(.2)$
\end{tabular} & $\begin{array}{l}2.0 \\
(.1)\end{array}$ & na \\
\hline Exp. $3 \& 7$ seawater & na & 7.82 & 31.314 & $\begin{array}{l}2106 \\
(10)\end{array}$ & $\begin{array}{l}364 \\
(6)\end{array}$ & $\begin{array}{l}1151 \\
(6)\end{array}$ & $\begin{array}{l}7.2 \\
(.2)\end{array}$ & $\begin{array}{l}7.4 \\
(.1)\end{array}$ & $\begin{array}{l}7.4 \\
(.1)\end{array}$ & $\begin{array}{l}8.9 \\
(.2)\end{array}$ & $\begin{array}{l}9.6 \\
(.4)\end{array}$ & 1.5 \\
\hline Exp. 3 & 0.83 & 9.25 & na & $\begin{array}{l}8630 \\
(15)\end{array}$ & \begin{tabular}{|l}
334 \\
$(5)$
\end{tabular} & $\begin{array}{l}1090 \\
(10)\end{array}$ & $\begin{array}{l}6.55 \\
(.04)\end{array}$ & $\begin{array}{l}6.8 \\
(.1)\end{array}$ & $\begin{array}{l}6.7 \\
(.1) \\
\end{array}$ & $\begin{array}{l}8.0 \\
(.1)\end{array}$ & na & 44 \\
\hline Exp. 3 & 3.2 & 9.14 & na & $\begin{array}{l}7320 \\
(25)\end{array}$ & $\begin{array}{l}331 \\
(3)\end{array}$ & $\begin{array}{l}1123 \\
(7)\end{array}$ & $\begin{array}{l}6.40 \\
(.01)\end{array}$ & $\begin{array}{l}6.63 \\
(.01)\end{array}$ & $\begin{array}{l}6.57 \\
(.03)\end{array}$ & $\begin{array}{l}7.30 \\
(.05)\end{array}$ & na & 34.3 \\
\hline$\overline{\operatorname{Exp} .3}$ & 31.8 & 8.17 & na & $\begin{array}{l}2716 \\
(6)\end{array}$ & $\begin{array}{l}254 \\
(5)\end{array}$ & $\begin{array}{l}1110 \\
(20)\end{array}$ & $\begin{array}{l}4.3 \\
(.1)\end{array}$ & $\begin{array}{l}14.1 \\
(.3)\end{array}$ & $\begin{array}{l}4.7 \\
(.1)\end{array}$ & $\begin{array}{l}8.1 \\
(.1)\end{array}$ & na & 2.7 \\
\hline Exp. 3 & 49.7 & 9.19 & na & $\begin{array}{l}8754 \\
(10)\end{array}$ & $\begin{array}{l}250 \\
(9)\end{array}$ & $\begin{array}{l}1110 \\
(40)\end{array}$ & $\begin{array}{l}8.7 \\
(.2)\end{array}$ & $\begin{array}{l}11.3 \\
(.4)\end{array}$ & $\begin{array}{l}4.6 \\
(.2)\end{array}$ & $\begin{array}{l}5.1 \\
(.2)\end{array}$ & na & 31.6 \\
\hline$\overline{\operatorname{Exp} .3}$ & 101.3 & 8.22 & 32.128 & $\begin{array}{l}3080 \\
(20)\end{array}$ & $\begin{array}{l}143 \\
(6)\end{array}$ & $\begin{array}{l}1100 \\
(50)\end{array}$ & $\begin{array}{l}5.2 \\
(.8)\end{array}$ & $\begin{array}{l}6.2 \\
(.2)\end{array}$ & $\begin{array}{l}2.5 \\
(.1)\end{array}$ & $\begin{array}{l}7.6 \\
(.2)\end{array}$ & na & 1.9 \\
\hline $\begin{array}{l}\text { Exp. } 4 \text { evaporated } \\
\text { sea water }\end{array}$ & 0 & na & 65.032 & na & $\begin{array}{l}710 \\
(3)\end{array}$ & $\begin{array}{l}2346 \\
(13)\end{array}$ & na & $\begin{array}{l}12.88 \\
(.03)\end{array}$ & na & $\begin{array}{l}28.8 \\
(.1)\end{array}$ & na & na \\
\hline Exp. 4, 5, $6 \mathrm{Na}_{2} \mathrm{CO}_{3}$ & 0 & na & na & na & $\begin{array}{l}3.3 \\
(.5) \\
\end{array}$ & \begin{tabular}{|l|}
79 \\
$(.01)$
\end{tabular} & na & $\begin{array}{l}0.014 \\
(.003) \\
\end{array}$ & na & $\begin{array}{l}100.6 \\
(.5)\end{array}$ & na & na \\
\hline $\begin{array}{l}\text { Exp. } 4 \text { high pump } \\
\text { rate }\end{array}$ & 0.1 & 9.29 & na & 5184 & \begin{tabular}{|l|}
344.5 \\
$(.7)$ \\
\end{tabular} & $\begin{array}{l}1138 \\
(4)\end{array}$ & na & $\begin{array}{l}6.49 \\
(.01)\end{array}$ & na & $\begin{array}{l}41.9 \\
(.11)\end{array}$ & na & 26.9 \\
\hline $\begin{array}{l}\text { Exp. } 4 \text { high pump } \\
\text { rate }\end{array}$ & 37.5 & 8.44 & 31.424 & 2686 & \begin{tabular}{|l|}
197.4 \\
$(.9)$
\end{tabular} & $\begin{array}{l}1160 \\
(6)\end{array}$ & na & $\begin{array}{l}3.43 \\
(.01)\end{array}$ & na & $\begin{array}{l}18.67 \\
(.03)\end{array}$ & na & 3.3 \\
\hline $\begin{array}{l}\text { Exp. } 5 \text { evaporated } \\
\text { sea water }\end{array}$ & 0 & na & 65.848 & na & $\begin{array}{l}803 \\
(2)\end{array}$ & $\begin{array}{l}2546 \\
(9)\end{array}$ & na & $\begin{array}{l}14.06 \\
(.04)\end{array}$ & na & $\begin{array}{l}14.63 \\
(.05)\end{array}$ & na & na \\
\hline Exp. 5, 6 sea water & 0 & 8.02 & na & na & $\begin{array}{l}360 \\
(1)\end{array}$ & $\begin{array}{l}1176 \\
(5)\end{array}$ & na & $\begin{array}{l}6.59 \\
(.003)\end{array}$ & na & $\begin{array}{l}6.25 \\
(.02)\end{array}$ & na & na \\
\hline $\begin{array}{l}\text { Exp. } 5 \text { mid pump } \\
\text { rate }\end{array}$ & 1.65 & 9.18 & na & 3176 & $\begin{array}{l}291 \\
(1)\end{array}$ & $\begin{array}{l}1188 \\
(6)\end{array}$ & na & $\begin{array}{l}5.11 \\
(.05)\end{array}$ & na & $\begin{array}{l}7.69 \\
(.01)\end{array}$ & na & 12 \\
\hline $\begin{array}{l}\text { Exp. } 5 \text { mid pump } \\
\text { rate }\end{array}$ & 112 & 8.57 & 32.788 & 4310 & $\begin{array}{l}56.1 \\
(.2)\end{array}$ & $\begin{array}{l}1233 \\
(4)\end{array}$ & na & $\begin{array}{l}.87 \\
(.001)\end{array}$ & na & $\begin{array}{l}15.81 \\
(.03)\end{array}$ & na & 1.8 \\
\hline $\begin{array}{l}\text { Exp. } 6 \text { evaporated } \\
\text { sea water }\end{array}$ & 0 & na & na & na & $\begin{array}{l}789 \\
(3)\end{array}$ & $\begin{array}{l}2525 \\
(12)\end{array}$ & na & $\begin{array}{l}13.75 \\
(.05)\end{array}$ & na & $\begin{array}{l}14.67 \\
(.04)\end{array}$ & na & na \\
\hline $\begin{array}{l}\text { Exp. } 6 \text { low pump } \\
\text { rate }\end{array}$ & 19 & 8.79 & na & 4228 & $\begin{array}{l}377 \\
(1)\end{array}$ & $\begin{array}{l}1206 \\
(6)\end{array}$ & na & $\begin{array}{l}6.92 \\
(.01)\end{array}$ & na & $\begin{array}{l}10.45 \\
(.03)\end{array}$ & na & 15.6 \\
\hline $\begin{array}{l}\text { Exp. } 6 \text { low pump } \\
\text { rate }\end{array}$ & 437 & 8.24 & 32.714 & 3371 & \begin{tabular}{|l|}
199.1 \\
$(.9)$
\end{tabular} & \begin{tabular}{|l}
1230 \\
$(5)$
\end{tabular} & na & $\begin{array}{l}3.26 \\
(.01)\end{array}$ & na & $\begin{array}{l}23.6 \\
(.1)\end{array}$ & na & 3 \\
\hline
\end{tabular}


Table EA 1 continued

\begin{tabular}{|c|c|c|c|c|c|c|c|c|c|c|c|c|}
\hline Experiment & time (hr) & $\mathrm{pH}$ & salinity & \begin{tabular}{|l|} 
alkalinity \\
(umol/kg)
\end{tabular} & \begin{tabular}{|l|}
$\mathrm{Ca}$ \\
$(\mathrm{ppm})$
\end{tabular} & $\begin{array}{l}\mathrm{Mg} \\
(\mathrm{ppm})\end{array}$ & $\begin{array}{l}84 \mathrm{Sr} \\
(\mathrm{ppm})\end{array}$ & $\begin{array}{l}86 \mathrm{Sr} \\
(\mathrm{ppm})\end{array}$ & $\begin{array}{l}88 \mathrm{Sr} \\
(\mathrm{ppm})\end{array}$ & $\begin{array}{l}\mathrm{Ba} \\
(\mathrm{ppb})\end{array}$ & $\begin{array}{l}\mathrm{Na} \\
(\mathrm{ppt})\end{array}$ & $\Omega$ \\
\hline Exp. 7 & 50.4 & 8.68 & na & $\begin{array}{l}5246 \\
(12)\end{array}$ & $\begin{array}{l}370 \\
(10)\end{array}$ & $\begin{array}{l}1200 \\
(20)\end{array}$ & $\begin{array}{l}6.96 \\
(.09)\end{array}$ & $\begin{array}{l}7.4 \\
(.2)\end{array}$ & $\begin{array}{l}7.3 \\
(.2)\end{array}$ & $\begin{array}{l}8.4 \\
(.1)\end{array}$ & na & 17.1 \\
\hline Exp. 7 & 73.7 & 8.17 & na & \begin{tabular}{|l}
3635 \\
$(4)$
\end{tabular} & $\begin{array}{l}320 \\
(10)\end{array}$ & $\begin{array}{l}1170 \\
(20)\end{array}$ & $\begin{array}{l}5.7 \\
(.4)\end{array}$ & $\begin{array}{l}6.3 \\
(.1)\end{array}$ & $\begin{array}{l}6.2 \\
(.3)\end{array}$ & $\begin{array}{l}6.6 \\
(.4)\end{array}$ & na & 4.6 \\
\hline Exp. 7 & 124.2 & 8.15 & na & $\begin{array}{l}3485 \\
(2)\end{array}$ & $\begin{array}{l}293 \\
(4)\end{array}$ & $\begin{array}{l}1160 \\
(20)\end{array}$ & $\begin{array}{l}.9 \\
(.3)\end{array}$ & $\begin{array}{l}19.4 \\
(.6)\end{array}$ & $\begin{array}{l}5.3 \\
(.1)\end{array}$ & $\begin{array}{l}5.9 \\
(.8)\end{array}$ & na & 3.8 \\
\hline$\overline{\operatorname{Exp} .7}$ & 152 & 8.12 & na & $\begin{array}{l}3389 \\
(7)\end{array}$ & $\begin{array}{l}280 \\
(4)\end{array}$ & $\begin{array}{l}1190 \\
(15)\end{array}$ & $\begin{array}{l}10.8 \\
(.2)\end{array}$ & $\begin{array}{l}17.7 \\
(.5)\end{array}$ & $\begin{array}{l}5.1 \\
(.2)\end{array}$ & $\begin{array}{l}5.36 \\
(.02) \\
\end{array}$ & na & 3.4 \\
\hline Exp. 7 & 166 & 8.06 & 33.5415 & $\begin{array}{l}3135 \\
(5)\end{array}$ & $\begin{array}{l}260 \\
(10)\end{array}$ & $\begin{array}{l}1160 \\
(50)\end{array}$ & $\begin{array}{l}10.1 \\
(.2)\end{array}$ & $\begin{array}{l}16.7 \\
(.3)\end{array}$ & $\begin{array}{l}4.8 \\
(.1)\end{array}$ & \begin{tabular}{|l}
5.16 \\
$(.04)$
\end{tabular} & na & 2.6 \\
\hline
\end{tabular}



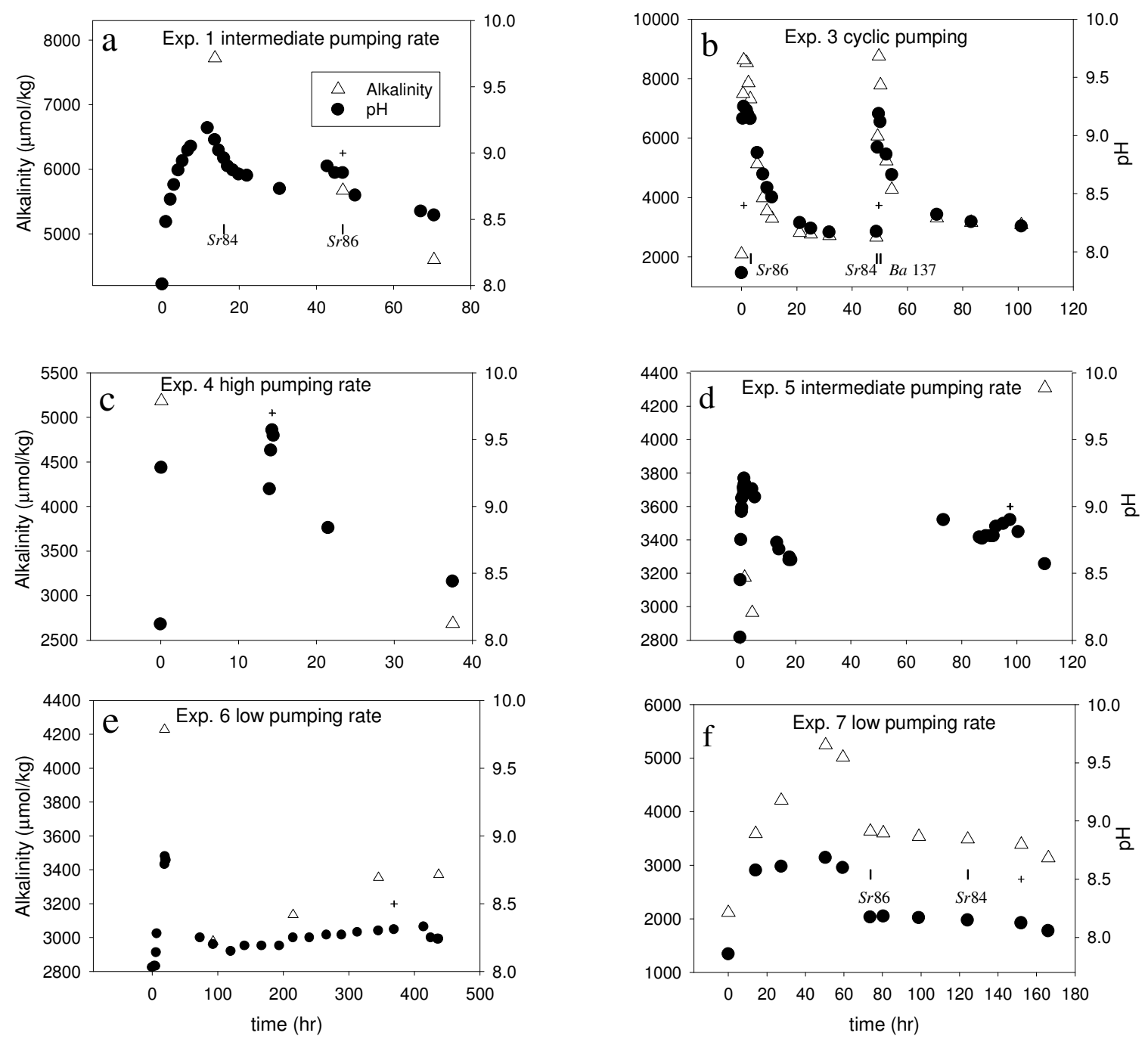

Figure EA-1. pH values (black circles, plotted on the right hand axis) and alkalinities $(\mu \mathrm{mol} / \mathrm{kg}$ ) (open triangles, plotted on the left hand axis) measured during synthetic precipitation experiments 1 (a), 3 (b), 4 (c), 5 (d), 6 (e), and 7 (d). The time at which pumping stopped is indicated by the cross on each plot. The timing of isotope spikes added for experiments 1,3 , and 7 is denoted by vertical lines labeled with the particular isotope added. The $\mathrm{x}$-axis shows time in hours for each experiment. Alkalinity and $\mathrm{pH}$ follow similar trends, with a peak shortly after the start of pumping, then dropping to a more stable value for the remainder of the run, except for the highest pumping rates (b, c), in which the $\mathrm{pH}$ remains high for the duration of pumping. Alkalinities in $\mathrm{d}$ are anomalous, and likely reflect precipitation during storage prior to alkalinity measurements. Sample storage may affect values for a, c, d, and e. Alkalinities for b and $\mathrm{f}$ were run within hours of collection, so sample storage is less of an issue. 

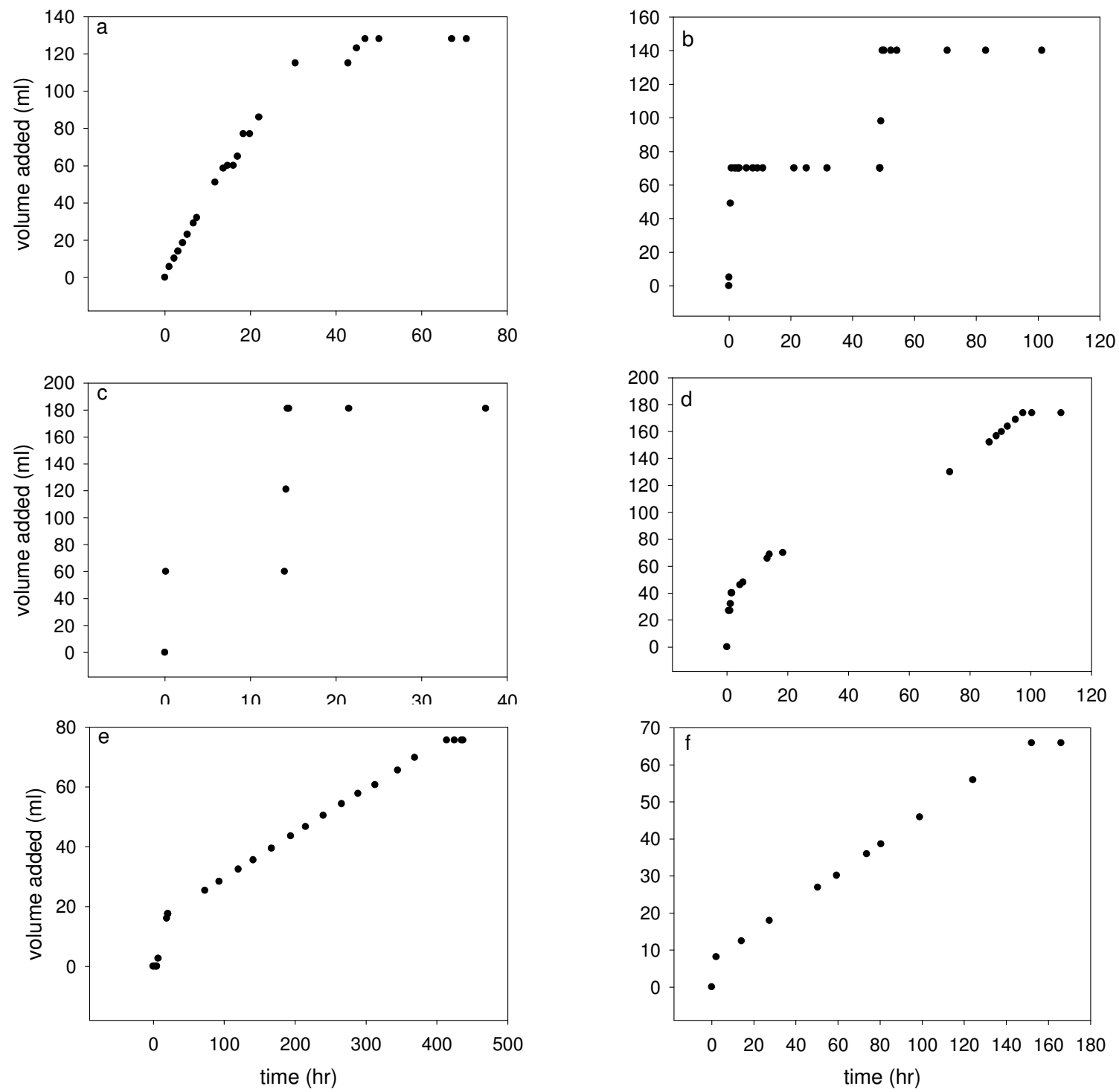

Figure EA-2. Volume of $0.04 \mathrm{M} \mathrm{Na}_{2} \mathrm{CO}_{3}$ (or equivalently, volume of evaporated seawater) added versus time for experiments 1 (a), 3 (b), 4 (c), 5 (d), 6 (e), and 7 (d). 


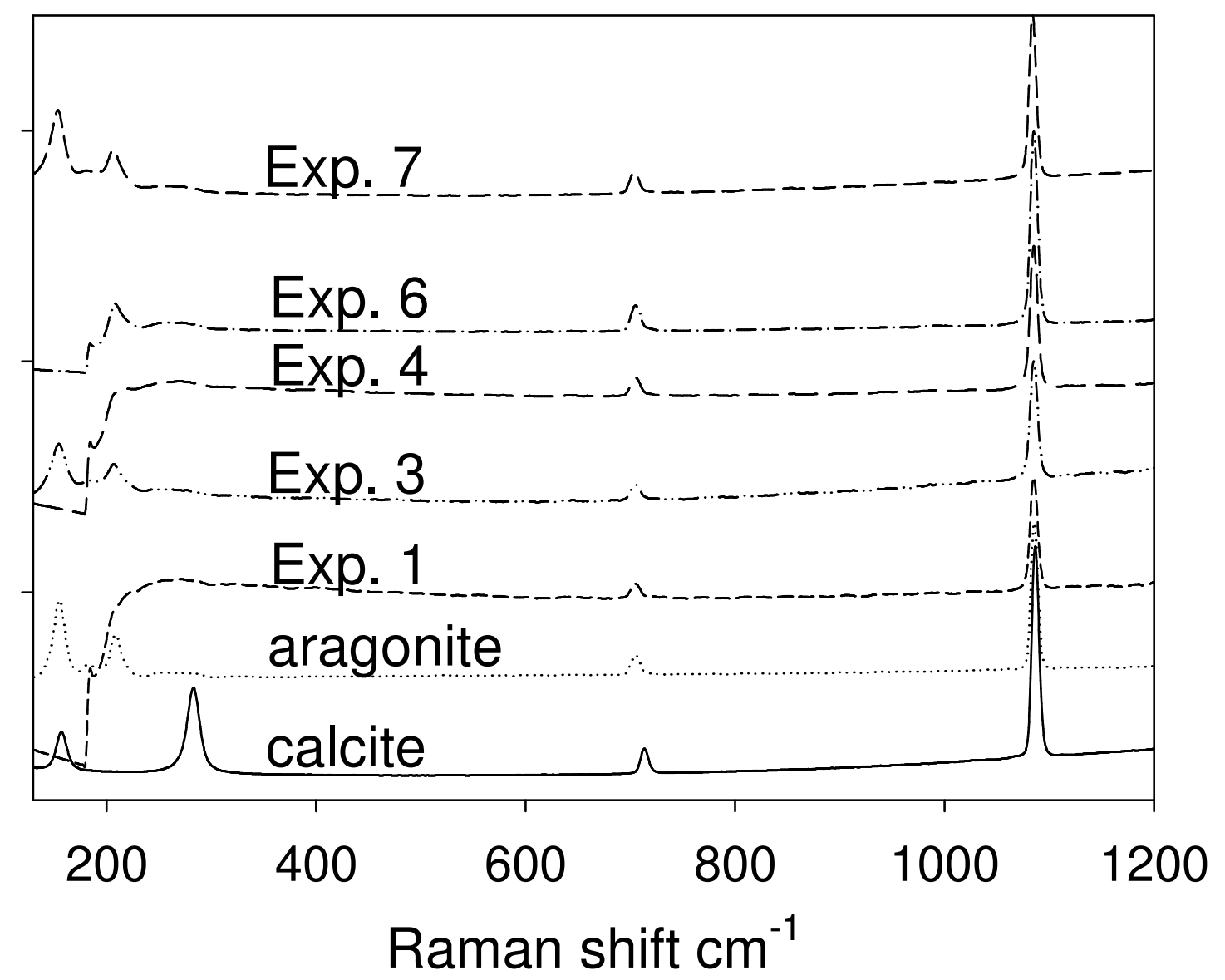

Figure EA-3. Raman spectra of mineral standards (aragonite and calcite), and synthetic precipitates. Both mineral aragonite and all experimental precipitates share a peak at 705 $\mathrm{cm}^{-1}$, suggesting the presence of aragonite in each of the experiments. Spectra are scaled such that the minimum y value of each spectrum is 0 , and the maximum is 1 . Offsets between spectra on the y axis are to separate spectra and do not reflect differences in baseline intensity. For Experiments 1, 4, and 6, the notch filter blocked spectra below $200 \mathrm{~cm}^{-1}$, thus the difference in this region reflects differences in the Raman microprobes used, and not differences between experiments. 

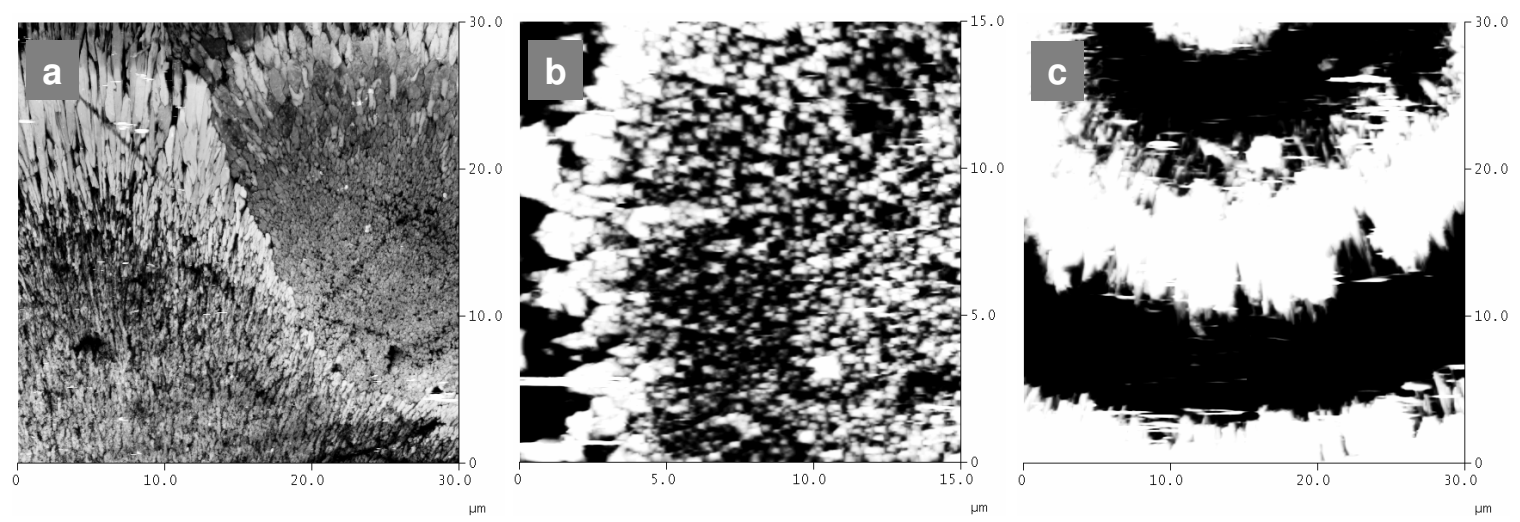

Figure EA-4. AFM height images of banded synthetic aragonite spherulites from experiment 2 prepared with different final etches. Spherulites were prepared with: colloidal silica (a), 30s acid etch (b), 60s acid etch (c). Height scales for all images are the same as in Fig. 4, except c, which is on a scale of 0 to $800 \mathrm{~nm}$. Note the loss of granular material and roughening of the surface with increasing etching durations. For synthetic aragonite, colloidal silica appears sufficient to give good images. 

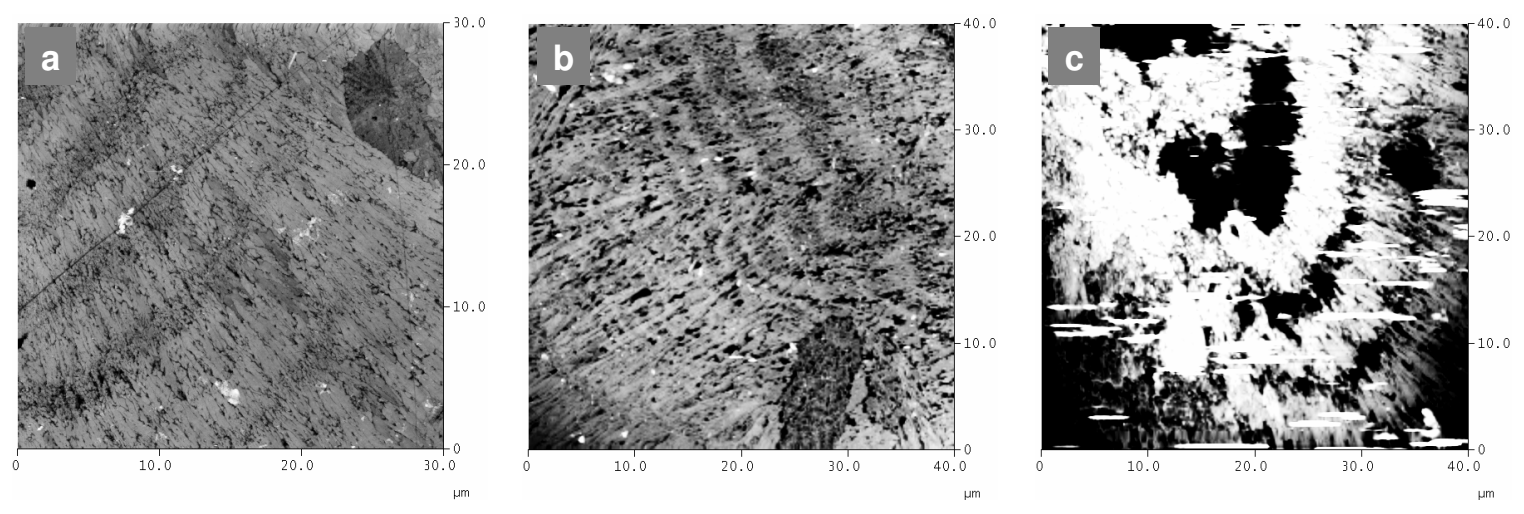

Figure EA-5. AFM height images of coral (Diploria), prepared with different final etches. Diploria labrynthiformis prepared with: colloidal silica (a), 50s acid etch (b), 50s acid etch followed by $16 \mathrm{hr}$ in distilled water (c). Height scales for all images are the same as in Fig. 4. Note the loss of granular material and roughening of the surface with increasing etching durations. A brief acid etch appears to improve contrast between features in corals. 

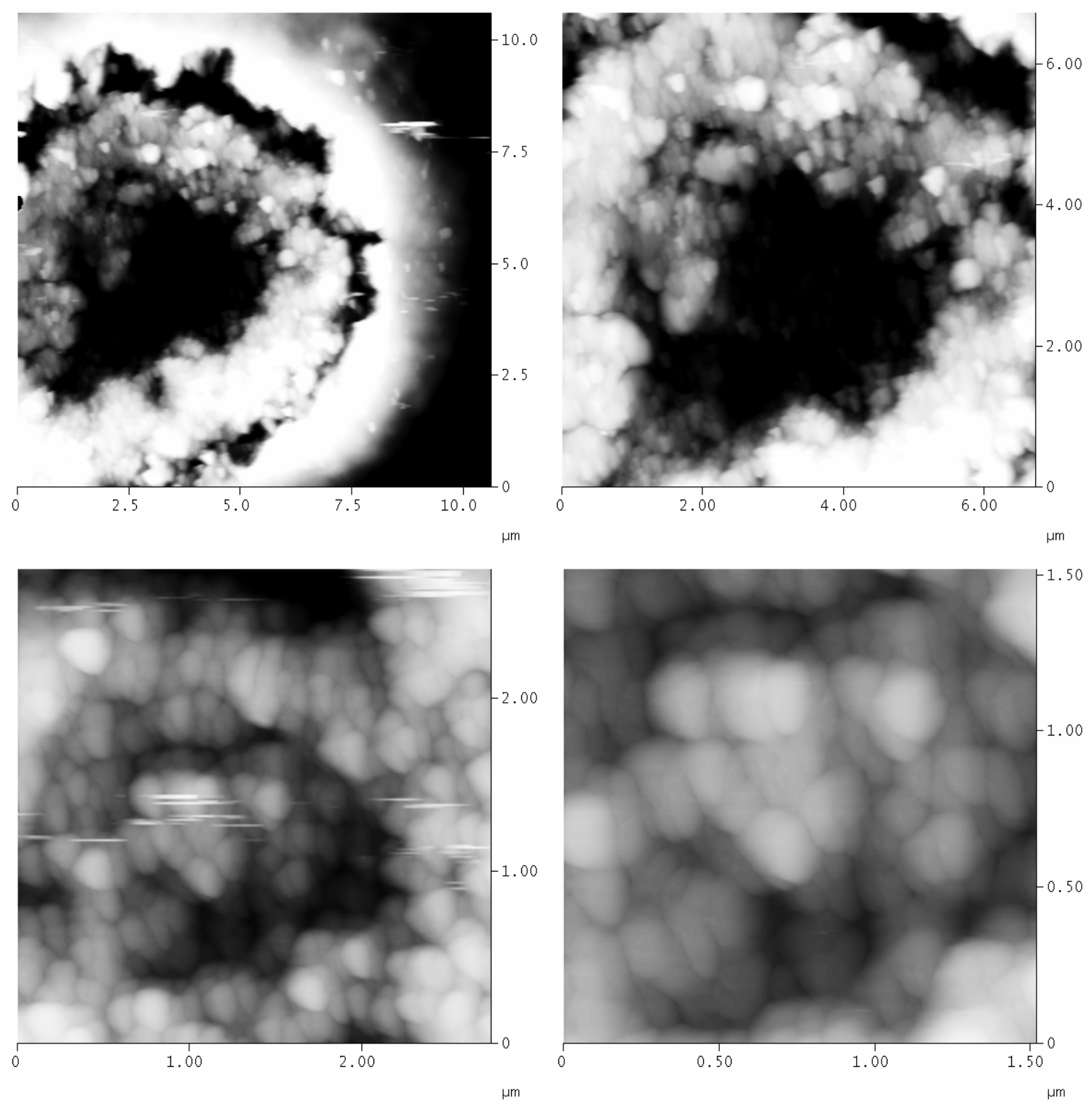

Figure EA-6. AFM images of experiment 4 taken at different scales. All images show the granular appearance of the surface of these grains at scales ranging from $10 \mu \mathrm{m}$ (upper left) to $1.5 \mu \mathrm{m}$ (lower right). Vertical scale is the same as in Fig. 4. 

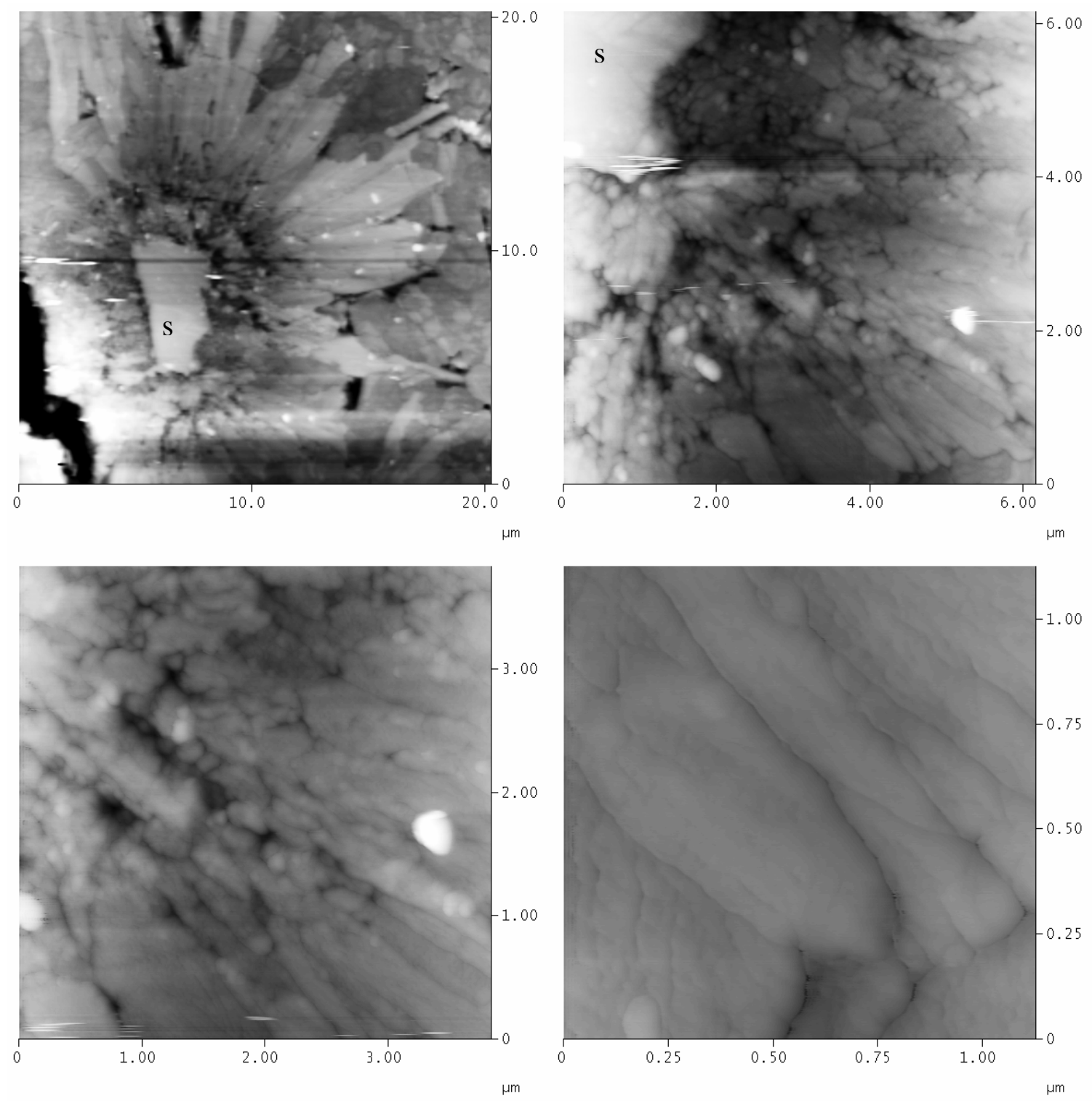

Figure EA-7. AFM images of experiment 6 taken at different scales. All images show the broad smooth fibers which characterize the low pumping rate experiments at scales ranging from $20 \mu \mathrm{m}$ (upper left) to $1 \mu \mathrm{m}$ (lower right). Granular materials are also seen near the center of these grains, and in the upper two images a seed crystal (s) is seen at the center, about which the grain nucleated. Vertical scale is the same as in Fig. 4. 


\section{References}

Gaetani G.A. and Cohen A.L. (2006) Element partitioning during precipitation of aragonite from seawater: a framework for understanding paleoproxies. Geochim. Cosmochim. Acta 70:4617-4634

Dickson A.G. (1990) Standard potential of the reaction: $\mathrm{AgCl}(\mathrm{s})+1 / 2 \mathrm{H} 2(\mathrm{~g})=\mathrm{Ag}(\mathrm{s})+$ $\mathrm{HCl}(\mathrm{aq})$, and the standard acidity constant of the ion HSO4- in synthetic seawater from 273.15 to $318.15 \mathrm{~K}$. Journal of Chemical Thermodynamics 22:113-127

Lewis E., and Wallace D.W.R. (1998) Program Developed for CO2 System Calculations. ORNL/CDIAC-105. Carbon Dioxide Information Analysis Center, Oak Ridge National Laboratory, U.S. Department of Energy, Oak Ridge, Tennessee.

Roy R.N., Roy L.N., Vogel K.M., Porter-Moore C., Pearson T., Good C.E., Millero F.J., and Campbell D.M. (1993) The dissociation constants of carbonic acid in seawater at salinities 5 to 45 and temperatures 0 to $45 \mathrm{deg}$ C. Marine Chemistry 44:249-267 
Additional discussion and experiments not included in manuscript

All M/Ca ratios are higher in the abiogenic aragonite than in the coral, which may reflect growth of the abiogenic aragonite at a slightly lower temperature than the coral or higher crystal growth rates. However, for $\mathrm{Ba} / \mathrm{Ca}$, ratios are much higher in abiogenic aragonite than they are in coral, this may in part be explained by Ba contaminating the $\mathrm{Na}_{2} \mathrm{CO}_{3}$ used to precipitate the experimental aragonite, so $\mathrm{Ba}$ concentration in the seawater used for abiogenic precipitation may be higher than that of the seawater in which the coral grew. Corals may also preferentially take up $\mathrm{Ca}$, reducing $\mathrm{M} / \mathrm{Ca}$ ratios in the calcification environment. The failure to see a significant difference for $\mathrm{Sr} / \mathrm{Ca}$ ratios between centers and fibers in the abiogenic precipitate may reflect the weak influence of growth rate on $\mathrm{Sr} / \mathrm{Ca}$ ratios (Gabitov et al., 2006), and the addition of Sr spikes slightly increased Sr concentrations in the fluid over time; or there may be additional factors influencing the ratios observed in the coral.

One factor which may influence elemental ratios in corals is the presence of organic materials which could serve as sites of heterogeneous nucleation allowing nucleation to occur at lower saturation states and potentially catalyzing crystal growth. If the saturation state for nucleation were reduced, this could reduce the saturation state at which initial crystal growth occurs, and an organic catalyst could influence elemental ratios - potentially lowering $\mathrm{M} / \mathrm{Ca}$ ratios. In the abiogenic experiments, organic materials and colloidal particles are present in the starting seawater which could similarly serve as sites of heterogeneous nucleation. Additionally the plastics in contact with the seawater and the air water interface all provide potential sites for heterogeneous 
nucleation. The effectiveness of different materials as sites of heterogeneous nucleation may differ, so different batches of seawater, different containers, or different corals may require different saturation states to achieve nucleation in a given period of time. So though saturation state has been shown to be closely linked to crystal morphology and composition, organic materials may play an important role as well.

Banding patterns in particular could be linked to organics. In studies of aragonite precipitation from artificial seawater, Suess and Futterer (1972) found humic substances to be a prerequisite for formation of spontaneously banded spherulites. Banded spherulites were found to incorporate thin ( 20nm) organic layers between thicker (microns) layers of fibrous aragonite Aragonite grown in the presence of humic substances had much higher $\mathrm{Mg}$ and $\mathrm{Sr}$ contents.

However, there are many mechanisms for generating banding patterns in crystal growth. Many minerals exhibit banding or oscillatory zoning. In the case of plagioclase, two major sources of banding have been identified. One involves changes in the bulk fluid composition, the other is suggested to result from cyclic changes in growth rate, potentially reflecting changes in a diffusive boundary layer surrounding the crystal. Both these forms of zoning are associated with marked changes in chemical composition, optical bands on the order of microns, and likely structural changes (Pearce and Kolisnik 1990). A similar model explains compositional bands as a result of growth from a highly supersaturated fluid with respect to more than one species, with a diffusive boundary layer, such that growth initially occurs incorporating primarily one species until the relative concentration of the second in the near surface environment builds up to the point 
that growth switches primarily to the second, which continues until the concentration of the first builds up again, thus generating regular compositional bands (Ortoleva 1990). A modification of these models has been proposed to explain Mn banding in calcite, in which interaction of growth rate and diffusion of $\mathrm{Mn}, \mathrm{H}$, and $\mathrm{Ca}$ ions generates oscillations in composition on scales of 100s of nm to 10s of microns (Reeder et al. 1990; Wang and Merino 1992). In agate, fine scale banded structures are associated with changes in crystal twisting, as well as composition (Wang and Merino 1990). Spherulitic crystal growth is commonly associated with banding patterns, with band spacing linked to crystal growth rate (Hutter and Bechhoefer 2000). In both cases, bands are visible to both light and AFM imaging, and have been suggested to stem from a change in crystal orientation. Thus, there are numerous potential means of generating banding patterns independent of organic materials or saturation state changes. And, mechanisms are not necessarily mutually exclusive, so multiple factors could play a role in determining the composition and morphology of coral skeletons.

The data presented here are consistent with saturation state being responsible for elemental and morphological variations within the coral skeleton. In zooxanthellate corals, the daily cycle in photosynthesis provides a mechanism which could be responsible for regular cycles in saturation state. In azooxanthellate corals, for which photosynthesis is unlikely to provide a means of raising saturation state on a regular cycle, regions of nucleation and fibrous growth still occur. Such variations may reflect the coral regulating the saturation state of the calcifying environment and periodically 
elevating the saturation state in specific regions to drive nucleation, however, other factors cannot be ruled out. 


\section{Comparison of PET and PTFE:}
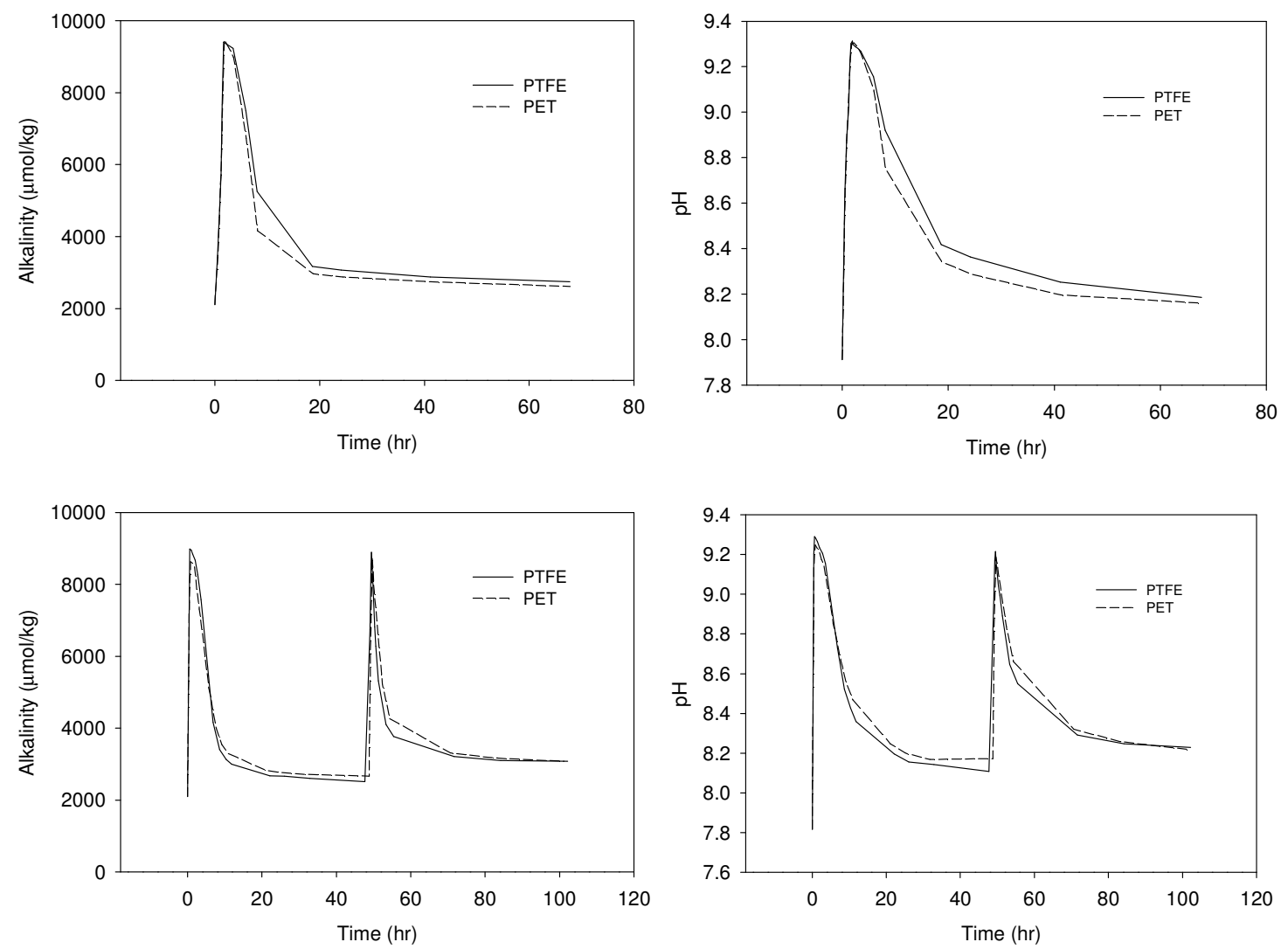

Figure A-1. Alkalinity and $\mathrm{pH}$ profiles for duplicate experiments carried out in PTFE and PET containers. Note similar profiles regardless of container type. 


\section{Additional References:}

Hutter JL, Bechhoefer J (2000) Banded spherulitic growth in a liquid crystal. J. Crystal Growth 217: 332-343

Ortoleva PJ (1990) Role of attachment kinetic feedback in the oscillatory zoning of crystals grown from melts. Earth Sci. rev. 29: 3-8

Pearce TH, Kolisnik AM (1990) Observations of plagioclase zoning using interference imaging. Earth Sci. rev. 29: 9-26

Reeder RJ, Fagioli RO, Meyers WJ (1990) Oscillatory zoning of Mn in solution-grown calcite crystals. Earth Sci. rev. 29: 39-46

Suess E, Futterer D (1972) Aragonitic ooids: experimental precipitation from seawater in the presence of humic acid. Sedimentology 19: 129-140

Wang Y, Merino E (1990) Self-organizational origin of agates: Banding, fiber twisting, composition, and dynamic crystallization model. Geochim. Cosmochim. Acta 54: $1627-1638$

Wang Y, Merino E (1992) Dynamic model of oscillatory zoning of trace elements in calcite: Double layer, inhibition, and self-organization. Geochim. Cosmochim. Acta 56: 587-596 
Appendix 2. Additional experiments conducted at Lizard Island Research Station and associated data.

In addition to those experiments described in Chapter 2, several additional experiments were conducted at the Lizard Island Research Station to determine the timing of band formation and how band formation is linked to environmental variables. In addition to the two zooxanthellate corals already discussed, one other zooxanthellate coral, Acropora $s p$. was used along with an azooxanthellate coral Tubastraea micranthus and a non scleractinian coral which hosts zooxanthellae Millepora sp.. Experiments using dyes were conducted on the additional species. But, in the dye based experiments there is the possibility that dye incorporation does not coincide with aragonite deposition - there may be portions of the skeleton which are entirely isolated from seawater and thus can form without incorporating dyes, or the dyes themselves may alter the calcification process. To avoid these possibilities, $\mathrm{Sr}$ and $\mathrm{Ba}$ isotope spikes were used to mark the skeleton in several incubations. A subset of experiments used elevated $\mathrm{pCO}_{2}$ to test the $\mathrm{CO}_{2}$ limitation hypothesis, while others manipulated light/dark cycles to distinguish whether band formation was tied to light/dark cycles or some independent variable.

Based on the model put forward in Chapter 2, azooxanthellate corals should not have bands of granular crystals formed on a daily basis - though both granular and fibrous crystals are found in azooxanthellate coral skeletons, fine scale bands are generally absent, and the timing of granular crystal formation is expected to differ from that seen in zooxanthellate corals. If band formation in zooxanthellate corals is linked to relief of $\mathrm{CO}_{2}$ limitation, then elevating external $\mathrm{CO}_{2}$ in the afternoon may relieve $\mathrm{CO}_{2}$ limitation and stimulate calcification (though this may be difficult to measure if the alkalinity source used is accumulated internal alkalinity as the model suggests). Daytime elevation of $\mathrm{CO}_{2}$ could shift the timing of band formation earlier in the day with a modest $\mathrm{CO}_{2}$ elevation or eliminate granular crystal formation altogether if levels are sufficient to prevent $\mathrm{CO}_{2}$ limitation. Elevation of external $\mathrm{CO}_{2}$ at dusk should reduce the time in which granular crystal formation can occur, potentially eliminating the formation of dark bands. Though not tested, reduced $\mathrm{CO}_{2}$ levels should be able to enhance band formation 
by increasing daytime $\mathrm{CO}_{2}$ limitation. Photosynthesis, and thus light is expected to be responsible for the formation of dark bands, thus it should be possible to change the timing of band formation by changing the light cycle - offsetting the light cycle by several hours from the natural cycle should induce a similar change in the timing of band formation. However, if the timing of band formation fails to track a change in the light cycle, factors other than photosynthesis may drive band formation. Similarly maintaining a coral entirely in the light or the dark should eliminate band formation. Though not tested, photosynthetic inhibitors or reduced daytime light (photosynthesis) levels could inhibit band formation by creating a situation in which $\mathrm{CO}_{2}$ is not limiting.

Coral collection

Corals were collected and mounted as described in Chapter 2, except a few additional sites were included - collection sites are specified for each parent colony in Table A-3.

\section{Staining}

Dyes

Dye incubations were carried out as described in Chapter 2, but additional intervals were included (Table A-1).

\section{Isotopes}

In addition to marking coral skeletons with dyes, several isotope $(\mathrm{Ba}(134,135,136,137$, $138)$ and $\operatorname{Sr}(84,86,87))$ spikes were used as well. Incubations were carried out in much the same manner as described for dye incubations, except coral fragments were transferred (underwater) to clean containers used for incubations so that alkalinity depletion measurements could be made over the same interval the spike was present. Each isotope used was purchased as a carbonate salt from Oak Ridge National Lab. Spikes were mixed with sufficient $\mathrm{HCl}$ to neutralize the carbonate and made up in distilled water to make stock solutions of which $50 \mu \mathrm{l}$ was used per liter of seawater. For $\mathrm{Sr}$ isotope spikes, this amounted to enough of the given $\mathrm{Sr}$ isotope to double the 
concentration of that particular isotope in the liter of seawater. For Ba isotope spikes, this amounted to sufficient $\mathrm{Ba}$ to almost double the total $\mathrm{Ba}$ concentration. The times at which each isotope spike was used are specified in Table A-2.

$\mathrm{CO}_{2}$ manipulation

A subset of incubations were conducted at elevated $\mathrm{CO}_{2}$ levels. To elevate $\mathrm{pCO}_{2}, \mathrm{a}$ rotameter (Alborg) was used to provide a controlled $\mathrm{CO}_{2}$ flow which mixed with air (supplied by an air pump) in a PET water bottle containing a phenol red solution (used as a visual indicator of reduced $\mathrm{pH}$ ). The air $\mathrm{CO}_{2}$ mix was then used to bubble individual incubations selected to receive elevated $\mathrm{CO}_{2}$. The water used for $\mathrm{CO}_{2}$ incubations was not pre-equilibrated with elevated $\mathrm{CO}_{2}$, thus $\mathrm{CO}_{2}$ levels start at ambient for all incubations, for a subset $\mathrm{CO}_{2}$ levels rise once $\mathrm{CO}_{2}$ bubbling is initiated. Details of each incubation are given in Table A-2,A-3.

\section{Water Chemistry}

Measurements of salinity, alkalinity, and $\mathrm{pH}$ were made periodically over the course of experiments. Salinity measurements were made using a conductivity probe (Hach), $\mathrm{pH}$ measurements were made using a spectrophotometer (see Chapter 4 for details), or were made on the NBS scale using an electrode. Alkalinity measurements were made using one of three methods - samples were either taken in Evergreen vials, stored refrigerated and returned to the US for measurement as described in Chapters 3 and 4, or $50 \mathrm{ml}$ samples were taken for immediate titration with $0.1 \mathrm{~N} \mathrm{HCl}$. Acid additions were made using a Hach Digital Titrator modified to accept a SGE $10 \mathrm{ml}$ gas-tight syringe. Changes in electrical potential were measured using a pH electrode. A non-linear least squares fit (based on DOE 1994) was used to determine alkalinity from titration data. Alkalinity depletion rates are calculated based salinity normalized differences in alkalinity between a control incubation (seawater without corals) and coral incubations. 


\section{Light}

Corals kept in the outdoor tank always received a natural light cycle. Indoor corals were initially kept on a $12 \mathrm{hr}$ light/dark cycle which coincided with the natural cycle (lighting provided by fluorescent lights suspended $\sim 30 \mathrm{~cm}$ above the corals). The lighting cycle was changed periodically for indoor corals to include periods of $24 \mathrm{hr}$ darkness, $24 \mathrm{hr}$ light, and a $12 \mathrm{hr}$ light/dark cycle offset from the natural cycle.

\section{Photographs}

In addition to photographing all corals as described in Chapter 4, a subset of corals were photographed every 10-15 min over the course of the experiment. To photograph corals

after dark, a red light (either LED based or red filtered) was used to provide illumination. Corals were photographed both indoors and outdoors to monitor patterns of tissue expansion/contraction.

\section{Buoyant weight}

Coral growth rates were estimated every $\sim 10$ days using the buoyant weight technique as described in Chapter 3.

\section{Harvesting}

At the end of experiments, corals were killed by briefly rinsing in fresh water followed by soaking in $\sim 3 \%$ bleach pre-equilibrated with aragonite. Once visible tissues was removed (30min to overnight depending on the species and how recently the bleach solution had been made up), specimens were rinsed briefly with fresh water and air dried. Specimens were killed at various times throughout the day/night (detailed in Table A-3) to allow crystal morphologies visible on the surface of the growing skeleton to be related to the crystal morphologies associated with stain-lines found in thin section. Thus SEM imaging of these specimens may provide another means to verify the link between crystal morphology and the time of day. 


\section{Loggers}

Hobo pendant loggers were deployed in an indoor aquarium used for the indoor experiments, an outdoor tank used for the outdoor experiments, and at the seawater intake to record the normal variation near the site of collection. Temperatures should be similar for both indoor and outdoor tanks as both are receiving a steady supply of seawater from the same source. Temperature differences between seawater within either tank and the intake may reflect the residence time of the seawater within header tanks which are used to maintain pressure in the running seawater system at the Lizard Island Research Station. Following deployment (start of each plot shown in Figures A-1-A-3), loggers were gradually overgrown with algae, periodically the loggers in the tanks were cleaned of overgrowth - this is associated with a gradual decline in light intensity followed by a sudden increase in intensity. Loggers were not always returned to precisely the same location following cleaning, and for the indoor tank, this leads to large shifts in light

intensity associated with the distance between the logger and the fluorescent light bulbs lighting the tanks.

Chlorophyll fluorescence was logged as well using a Wet Labs fluorimeter. 
Table A-1. Timing of dye spikes, dye, and dye concentration used. Spikes designated by the same group letter were applied to the same corals. Dyes used were: oxytetracycline (OTC), alizarin, alizarin complexone (AC), and calcein. The number of corals indicates the total number of specimens used.

\begin{tabular}{|l|l|l|l|l|l|}
\hline Group & Time in & Time out & $\begin{array}{l}\text { \# corals } \\
\text { stained }\end{array}$ & Dye & Dye concentration \\
\hline a & $12 / 21 / 0718: 00$ & $12 / 22 / 076: 15$ & 6 & OTC & $40 \mathrm{mg} / \mathrm{L}$ \\
\hline a & $12 / 22 / 0718: 00$ & $12 / 23 / 076: 00$ & 6 & OTC & $40 \mathrm{mg} / \mathrm{L}$ \\
\hline b & $12 / 23 / 0718: 30$ & $12 / 24 / 076: 15$ & 5 & alizarin & $10 \mathrm{mg} / \mathrm{L}$ \\
\hline b & $12 / 24 / 0718: 50$ & $12 / 25 / 076: 40$ & 5 & alizarin & $8 \mathrm{mg} / \mathrm{L}$ \\
\hline c & $12 / 26 / 0719: 00$ & $12 / 27 / 077: 00$ & 4 & AC & $10 \mathrm{mg} / \mathrm{L}$ \\
\hline c & $12 / 27 / 0718: 25$ & $12 / 28 / 076: 40$ & 4 & AC & $10 \mathrm{mg} / \mathrm{L}$ \\
\hline d & $12 / 26 / 0719: 10$ & $12 / 27 / 077: 00$ & 5 & calcein & $10 \mathrm{mg} / \mathrm{L}$ \\
\hline d & $12 / 27 / 0718: 25$ & $12 / 28 / 076: 40$ & 5 & calcein & $10 \mathrm{mg} / \mathrm{L}$ \\
\hline e & $1 / 20 / 0818: 00$ & $1 / 21 / 088: 15$ & 4 & calcein & $10 \mathrm{mg} / \mathrm{L}$ \\
\hline e & $1 / 21 / 088: 30$ & $1 / 21 / 0817: 00$ & 4 & alizarin & $5 \mathrm{mg} / \mathrm{L}$ \\
\hline f & $1 / 22 / 0818: 20$ & $1 / 23 / 088: 30$ & 4 & calcein & $10 \mathrm{mg} / \mathrm{L}$ \\
\hline f & $1 / 23 / 088: 50$ & $1 / 23 / 0818: 30$ & 4 & AC & $10 \mathrm{mg} / \mathrm{L}$ \\
\hline g & $1 / 23 / 0819: 40$ & $1 / 24 / 088: 00$ & 5 & calcein & $10 \mathrm{mg} / \mathrm{L}$ \\
\hline g & $1 / 24 / 088: 35$ & $1 / 24 / 0817: 00$ & 5 & alizarin & $5 \mathrm{mg} / \mathrm{L}$ \\
\hline g & $1 / 24 / 0818: 30$ & $1 / 25 / 087: 50$ & 5 & AC & $10 \mathrm{mg} / \mathrm{L}$ \\
\hline h & $1 / 17 / 0818: 20$ & $1 / 18 / 086: 15$ & 2 & calcein & $8 \mathrm{mg} / \mathrm{L}$ \\
\hline h & $1 / 18 / 086: 40$ & $1 / 18 / 0817: 30$ & 2 & alizarin & $5 \mathrm{mg} / \mathrm{L}$ \\
\hline i & $1 / 17 / 0818: 20$ & $1 / 18 / 086: 15$ & 2 & calcein & $8 \mathrm{mg} / \mathrm{L}$ \\
\hline i & $1 / 18 / 086: 40$ & $1 / 18 / 0817: 30$ & 2 & AC & $10 \mathrm{mg} / \mathrm{L}$ \\
\hline
\end{tabular}


Table A-2. Timing of isotope spikes, isotope, and lighting or $\mathrm{CO}_{2}$ condition used (levels are ambient for normal $\mathrm{CO}_{2}$, light in phase with natural cycle, $\mathrm{CO}_{2}$ for elevated $\mathrm{CO}_{2}$ and the time at which $\mathrm{CO}_{2}$ bubbling started, light for $24 \mathrm{~h}$ light, dark for $24 \mathrm{~h}$ dark, or altered with a time to indicate an altered $12 \mathrm{hr}$ light/dark cycle with the light phase starting at the specified time. Spikes designated by the same group were applied to the same corals, groups starting with "in" indicates corals were indoor, "out" indicates outdoor. The number of corals indicates the total number of specimens used. For indoor runs where the number of corals exceeds 7 , all corals were spiked together in a common tank rather than isolated individually.

\begin{tabular}{|c|c|c|c|c|c|}
\hline Group & Time in & Time out & \# corals & Isotope & Condition \\
\hline in1 & 11/10/07 21:00 & 11/11/07 5:00 & 6 & Sr 84 & ambient \\
\hline in1 & 11/11/07 9:40 & $11 / 11 / 07$ 18:50 & 6 & Sr 86 & ambient \\
\hline in2 & 11/13/07 18:50 & 11/14/07 5:30 & 6 & Sr 84 & ambient \\
\hline in2 & 11/14/07 9:00 & 11/14/07 18:00 & 6 & Sr 86 & ambient \\
\hline in2 & 11/14/07 20:00 & 11/15/07 6:00 & 6 & Ba 135 & ambient \\
\hline in 3 & 11/20/07 9:00 & 11/20/07 18:15 & 6 & Sr 84 & ambient \\
\hline in3 & $11 / 20 / 0720: 30$ & 11/21/07 6:15 & 6 & Sr 86 & ambient \\
\hline in3 & $11 / 21 / 07$ 10:00 & $11 / 21 / 07$ 17:40 & 6 & Ba 135 & ambient \\
\hline in3 & $11 / 21 / 0721: 30$ & 11/22/07 16:30 & 6 & Sr 87 & ambient \\
\hline in3 & 11/22/07 9:30 & 11/22/07 16:30 & 6 & Ba 138 & ambient \\
\hline in4 & 11/26/07 8:45 & 11/27/07 6:30 & 6 & Sr 87 & ambient \\
\hline in4 & 11/26/07 19:00 & 11/27/07 6:30 & 6 & Ba 135 & ambient \\
\hline in4 & $11 / 27 / 079: 20$ & 11/27/07 18:20 & 6 & Sr 84 & ambient \\
\hline in4 & 11/27/07 20:00 & 11/28/07 6:00 & 6 & Sr 86 & ambient \\
\hline in5 & 12/24/07 19:10 & 12/25/07 6:15 & 13 & Sr 84 & ambient \\
\hline in5 & $12 / 25 / 07 \quad 18: 30$ & 12/26/07 7:20 & 13 & Sr 86 & ambient \\
\hline in5 & $12 / 26 / 07$ 19:30 & 12/27/07 6:40 & 13 & Sr 87 & ambient \\
\hline in5 & 12/27/07 7:00 & 12/27/07 18:00 & 13 & Ba 137 & ambient \\
\hline in6 & $12 / 26 / 07$ 19:30 & 12/27/07 17:00 & 7 & Sr 84 & ambient \\
\hline in6 & 12/27/07 6:50 & 12/27/07 17:00 & 7 & Ba 137 & ambient \\
\hline in6 & 12/27/07 19:00 & 12/28/07 6:30 & 7 & Sr 87 & ambient \\
\hline in7 & 12/28/07 7:00 & 12/28/07 19:00 & 18 & Sr 86 & ambient \\
\hline in7 & $12 / 29 / 077: 15$ & 12/29/07 19:00 & 18 & Sr 84 & ambient \\
\hline in7 & $12 / 29 / 07 \quad 17: 30$ & 12/29/07 19:00 & 18 & $\mathrm{Ba} 134$ & ambient \\
\hline in7 & 12/30/07 7:00 & 12/30/07 20:30 & 18 & Ba 135 & ambient \\
\hline in7 & 12/30/07 13:40 & 12/30/07 20:30 & 18 & Ba 136 & ambient \\
\hline in7 & $1 / 2 / 0812: 50$ & 1/3/07 1:00 & 18 & Ba 138 & ambient \\
\hline in7 & 1/2/08 19:20 & 1/3/07 1:00 & 18 & Sr 84 & ambient \\
\hline in7 & $1 / 3 / 082: 00$ & $1 / 3 / 08$ 14:30 & 18 & Ba 137 & ambient \\
\hline in7 & 1/5/08 0:00 & $1 / 5 / 0812: 30$ & 18 & Sr 86 & altered 0:00 \\
\hline in7 & $1 / 5 / 0823: 45$ & $1 / 6 / 08$ 12:40 & 18 & Sr 87 & altered 23:50 \\
\hline in7 & $1 / 6 / 0823: 45$ & 1/7/08 12:05 & 18 & $\mathrm{Ba} 136$ & altered $23: 50$ \\
\hline in7 & 1/7/08 6:10 & 1/7/08 12:05 & 18 & Sr 87 & altered $23: 50$ \\
\hline
\end{tabular}




\begin{tabular}{|c|c|c|c|c|c|}
\hline in7 & 1/8/08 12:00 & $1 / 8 / 0823: 15$ & 18 & $\mathrm{Ba} 134$ & altered $23: 50$ \\
\hline in7 & 1/9/08 12:00 & $1 / 9 / 0823: 50$ & 18 & Sr 86 & altered 23:10 \\
\hline in7 & 1/10/08 18:30 & $1 / 10 / 0823: 40$ & 18 & Sr 84 & altered $23: 50$ \\
\hline in7 & $1 / 12 / 0823: 45$ & $1 / 13 / 0814: 10$ & 18 & Ba 137 & light \\
\hline in7 & $1 / 13 / 08$ 14:15 & 1/14/08 10:40 & 18 & Ba 137 & light \\
\hline in7 & 1/13/08 14:15 & 1/14/08 10:40 & 18 & Sr 87 & light \\
\hline in7 & 1/17/08 9:00 & $1 / 17 / 0821: 55$ & 18 & Ba 138 & dark \\
\hline in7 & $1 / 17 / 0822: 25$ & 1/18/08 8:00 & 18 & $\mathrm{Ba} 138$ & dark \\
\hline in7 & 1/17/08 22:25 & 1/18/08 8:00 & 18 & Sr 84 & dark \\
\hline out1 & 12/5/07 6:00 & $12 / 5 / 07$ 18:30 & 6 & Sr 84 & ambient \\
\hline out1 & 12/5/07 19:30 & 12/6/07 17:00 & 6 & Sr 86 & ambient \\
\hline out1 & 12/6/07 7:00 & 12/6/07 17:00 & 6 & $\mathrm{Ba} 135$ & ambient \\
\hline out1 & 12/6/07 18:00 & 12/7/07 6:00 & 6 & Sr 87 & ambient \\
\hline out2 & 12/8/07 18:40 & 12/9/07 16:00 & 6 & Sr 84 & ambient \\
\hline out2 & 12/9/07 6:00 & 12/9/07 16:00 & 6 & $\mathrm{Ba} 135$ & ambient \\
\hline out2 & 12/9/07 18:00 & 12/10/07 6:00 & 6 & Sr 86 & ambient \\
\hline out2 & $12 / 10 / 077: 30$ & 12/10/07 17:00 & 6 & Sr 87 & ambient \\
\hline out3 & 12/16/07 6:30 & 12/16/07 18:00 & 6 & Sr 84 & ambient \\
\hline out3 & 12/16/07 18:00 & 12/17/07 16:30 & 6 & Sr 86 & ambient \\
\hline out3 & 12/17/07 6:00 & 12/17/07 16:30 & 6 & $\mathrm{Ba} 135$ & ambient \\
\hline out3 & 12/17/07 17:30 & 12/18/07 6:00 & 6 & Sr 87 & ambient \\
\hline out4 & 12/18/07 18:00 & 12/19/07 16:15 & 7 & Sr 84 & ambient \\
\hline out4 & 12/19/07 6:00 & 12/19/07 16:15 & 7 & $\mathrm{Ba} 135$ & ambient \\
\hline out4 & 12/19/07 18:40 & 12/20/07 6:00 & 7 & Sr 86 & ambient \\
\hline out4 & $12 / 20 / 077: 40$ & $12 / 20 / 07$ 17:45 & 7 & Sr 87 & ambient \\
\hline out5 & 12/28/07 18:30 & 12/29/07 7:00 & 14 & Sr 86 & ambient \\
\hline out5 & 12/29/07 9:30 & 12/29/07 17:30 & 14 & Sr 87 & ambient \\
\hline out5 & 12/29/07 19:20 & 12/30/07 18:15 & 14 & Sr 84 & ambient \\
\hline out5 & 12/30/07 6:15 & 12/30/07 18:15 & 14 & Ba 135 & ambient \\
\hline out5 & 12/30/07 13:10 & 12/30/07 18:15 & 14 & $\mathrm{Ba} 136$ & ambient \\
\hline out6 & 12/31/07 16:40 & $1 / 1 / 087: 20$ & 7 & Sr 86 & ambient \\
\hline out6 & 12/31/07 19:05 & 1/1/08 7:20 & 7 & $\mathrm{Ba} 136$ & ambient \\
\hline out6 & $1 / 1 / 087: 55$ & 1/1/08 18:40 & 7 & Sr 87 & ambient \\
\hline out6 & $1 / 1 / 08$ 13:00 & $1 / 1 / 08$ 18:40 & 7 & $\mathrm{Ba} 138$ & ambient \\
\hline out6 & 1/1/08 19:15 & 1/2/08 19:00 & 7 & Sr 84 & ambient \\
\hline out6 & 1/2/08 6:00 & 1/2/08 19:00 & 7 & Ba 137 & ambient \\
\hline out7 & $1 / 3 / 08$ 18:40 & 1/4/08 16:00 & 7 & Sr 84 & ambient \\
\hline out7 & $1 / 4 / 086: 20$ & 1/4/08 16:00 & 7 & $\mathrm{Ba} 135$ & ambient \\
\hline out7 & $1 / 4 / 0817: 30$ & $1 / 5 / 087: 20$ & 7 & Sr 86 & ambient \\
\hline out7 & $1 / 5 / 087: 40$ & 1/5/08 17:10 & 7 & Sr 87 & ambient \\
\hline out8 & $1 / 6 / 08$ 17:40 & 1/7/08 16:00 & 13 & Ba 137 & ambient \\
\hline out8 & 1/7/08 6:20 & 1/7/08 16:00 & 13 & Sr 87 & ambient \\
\hline
\end{tabular}




\begin{tabular}{|c|c|c|c|c|c|}
\hline out8 & 1/7/08 18:50 & 1/8/08 6:10 & 13 & Sr 86 & ambient \\
\hline out8 & $1 / 8 / 086: 50$ & 1/8/08 17:10 & 13 & Sr 84 & ambient \\
\hline out8 & 1/8/08 12:15 & 1/8/08 17:10 & 13 & Ba 135 & ambient \\
\hline out9 & $1 / 8 / 08 \quad 17: 55$ & 1/9/08 17:40 & 7 & Sr 87 & ambient \\
\hline out9 & 1/9/08 6:08 & 1/9/08 17:40 & 7 & Ba 134 & ambient \\
\hline out9 & 1/9/08 11:45 & 1/9/08 17:40 & 7 & Ba 137 & ambient \\
\hline out9 & 1/9/08 18:10 & 1/10/08 6:50 & 7 & Sr 86 & ambient \\
\hline out 9 & 1/10/08 7:20 & 1/10/08 18:00 & 7 & Sr 84 & ambient \\
\hline out9 & 1/10/08 12:06 & 1/10/08 18:00 & 7 & Ba 135 & ambient \\
\hline out10 & 1/11/08 7:00 & $1 / 11 / 0818: 50$ & 4 & Sr 87 & ambient \\
\hline out10 & $1 / 11 / 08$ 19:20 & 1/12/08 17:40 & 4 & Sr 86 & ambient \\
\hline out 10 & $1 / 12 / 087: 50$ & 1/12/08 17:40 & 4 & Ba 137 & ambient \\
\hline out10 & 1/12/08 18:20 & 1/13/08 7:30 & 4 & Sr 84 & ambient \\
\hline out11 & 1/11/08 7:00 & $1 / 11 / 0818: 50$ & 3 & Sr 87 & ambient \\
\hline out11 & 1/11/08 12:15 & 1/11/08 18:50 & 3 & Ba 134 & ambient \\
\hline out11 & 1/11/08 19:20 & $1 / 12 / 08$ 17:40 & 3 & Sr 86 & ambient \\
\hline out11 & $1 / 12 / 087: 50$ & 1/12/08 17:40 & 3 & Ba 137 & ambient \\
\hline out11 & 1/12/08 18:20 & 1/13/08 7:30 & 3 & Sr 84 & ambient \\
\hline out12 & 1/13/08 18:30 & 1/14/08 18:00 & 7 & Sr 87 & ambient \\
\hline out12 & $1 / 14 / 08$ 7:20 & 1/14/08 18:00 & 7 & Ba 137 & ambient \\
\hline out12 & $1 / 14 / 0818: 30$ & 1/15/08 8:00 & 7 & Sr 86 & ambient \\
\hline out12 & 1/15/08 8:20 & $1 / 15 / 08$ 19:40 & 7 & Sr 84 & ambient \\
\hline out12 & 1/15/08 12:15 & 1/15/08 19:40 & 7 & Ba 135 & ambient \\
\hline out13 & $1 / 18 / 087: 40$ & 1/18/08 16:20 & 7 & Ba 138 & ambient $/ \mathrm{CO}_{2}$ \\
\hline out13 & $1 / 18 / 0811: 55$ & 1/18/08 16:20 & 7 & Sr 86 & $\mathrm{CO}_{2} 11: 46$ \\
\hline out13 & $1 / 18 / 0816: 55$ & 1/19/08 15:30 & 7 & Ba 136 & ambient \\
\hline out13 & 1/19/08 8:00 & 1/19/08 15:30 & 7 & Sr 87 & ambient \\
\hline out13 & 1/19/08 15:56 & 1/19/08 20:00 & 7 & Sr 84 & $\mathrm{CO}_{2} 16: 00$ \\
\hline out13 & 1/19/08 20:00 & 1/20/08 6:30 & 7 & Sr 84 & ambient \\
\hline out13 & 1/19/08 20:00 & 1/20/08 6:30 & 7 & Ba 137 & ambient \\
\hline out14 & 1/20/08 8:00 & 1/20/08 16:30 & 7 & Ba 138 & ambient/ $\mathrm{CO}_{2}$ \\
\hline out14 & $1 / 20 / 0811: 58$ & $1 / 20 / 0816: 30$ & 7 & Sr 86 & $\mathrm{CO}_{2} 12: 03$ \\
\hline out14 & $1 / 20 / 0816: 50$ & $1 / 21 / 08$ 15:10 & 7 & Ba 136 & ambient \\
\hline out14 & 1/21/08 8:10 & 1/21/08 15:10 & 7 & Sr 87 & ambient \\
\hline out14 & $1 / 21 / 08$ 15:53 & 1/21/08 20:00 & 7 & Sr 84 & $\mathrm{CO}_{2} 15: 55$ \\
\hline out14 & 1/21/08 20:00 & 1/22/08 7:00 & 7 & Sr 84 & ambient \\
\hline out14 & 1/21/08 20:00 & 1/22/08 7:00 & 7 & Ba 137 & ambient \\
\hline out15 & $1 / 22 / 08$ 8:38 & 1/22/08 16:15 & 6 & Ba 138 & ambient $/ \mathrm{CO}_{2}$ \\
\hline out15 & $1 / 22 / 08$ 11:45 & $1 / 22 / 0816: 15$ & 6 & Sr 86 & $\mathrm{CO}_{2} 11: 53$ \\
\hline out15 & $1 / 22 / 08 \quad 16: 35$ & 1/23/08 16:40 & 6 & Ba 136 & ambient \\
\hline out15 & 1/23/08 9:06 & $1 / 23 / 0816: 40$ & 6 & Sr 87 & ambient \\
\hline out15 & $1 / 23 / 0816: 55$ & $1 / 23 / 08$ 19:45 & 6 & Sr 84 & $\mathrm{CO}_{2} 16: 59$ \\
\hline
\end{tabular}




\begin{tabular}{|l|l|l|l|l|l|}
\hline out15 & $1 / 23 / 0819: 45$ & $1 / 24 / 0810: 20$ & 6 & $\mathrm{Sr} 84$ & ambient \\
\hline out15 & $1 / 23 / 0819: 45$ & $1 / 24 / 0810: 20$ & 6 & $\mathrm{Ba} 137$ & ambient \\
\hline out16 & $1 / 22 / 088: 38$ & $1 / 22 / 0816: 15$ & 1 & $\mathrm{Ba} 138$ & ambient/CO \\
\hline out16 & $1 / 22 / 0811: 45$ & $1 / 22 / 0816: 15$ & 1 & $\mathrm{Sr} 86$ & $\mathrm{CO}_{2} 11: 53$ \\
\hline out16 & $1 / 22 / 0816: 35$ & $1 / 23 / 0816: 40$ & 1 & $\mathrm{Ba} 136$ & ambient \\
\hline out16 & $1 / 23 / 089: 06$ & $1 / 23 / 0816: 40$ & 1 & $\mathrm{Ba} 135$ & ambient \\
\hline out16 & $1 / 23 / 0816: 55$ & $1 / 23 / 0819: 45$ & 1 & $\mathrm{Sr} 84$ & $\mathrm{CO}_{2} 16: 59$ \\
\hline out16 & $1 / 23 / 0819: 45$ & $1 / 24 / 0810: 20$ & 1 & $\mathrm{Sr} 84$ & ambient \\
\hline out16 & $1 / 23 / 0819: 45$ & $1 / 24 / 0810: 20$ & 1 & $\mathrm{Ba} 137$ & ambient \\
\hline out17 & $1 / 25 / 089: 06$ & $1 / 25 / 0818: 25$ & 4 & $\mathrm{Ba} 135$ & ambient \\
\hline out18 & $1 / 25 / 089: 06$ & $1 / 25 / 0814: 35$ & 4 & $\mathrm{Ba} 135$ & $\mathrm{CO}_{2} 11: 25$ \\
\hline out18 & $1 / 25 / 0814: 35$ & $1 / 25 / 0818: 25$ & 4 & $\mathrm{Ba} 135$ & ambient \\
\hline
\end{tabular}


Table A-3. Collection sites for each coral, fragments obtained from each coral, treatments received by each fragment (per Table A-1, A-2), time at which each fragment was bleached and whether polyps were open or closed when the fragment was bleached.

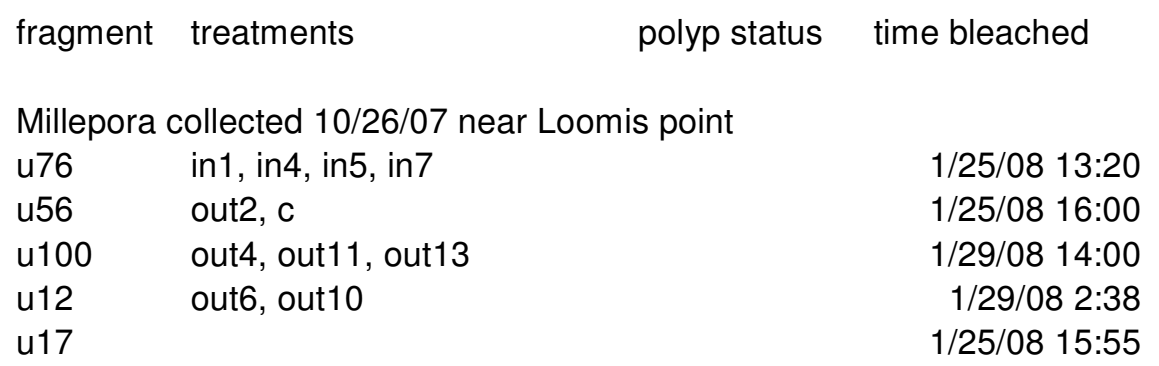


Acropora millepora collected 10/27/07 near seawater intake

$\begin{array}{lllr}\text { u81 } & \text { c } & \text { open } & 1 / 28 / 0816: 00 \\ \text { u68 } & \text { in4, in5, in7 } & & 1 / 25 / 080: 00 \\ \text { u58 } & \text { out2 } & \text { open } & 1 / 28 / 0814: 05 \\ \text { u69 } & \text { out3, out9, out13 } & \text { partly open } & 1 / 28 / 0823: 55 \\ \text { u83 } & \text { out3, out9, out13 } & \text { partly open } & 1 / 28 / 0823: 55\end{array}$

Tubastraea micranthus 10/28/07 6-9m depth near North Point

$\begin{array}{lllr}\text { u49 } & \text { in2, in5, in7 } & \text { open } & 1 / 28 / 0822: 40 \\ \text { u87 } & \text { out1, b } & \text { closed } & 1 / 17 / 0814: 30 \\ \text { u27 } & \text { out5, i } & \text { open } & 1 / 28 / 0822: 50 \\ \text { u9 } & \text { out5, out8 } & \text { closed } & 1 / 17 / 0814: 15 \\ \text { h93 } & & & 12 / 2 / 0723: 30 \\ \text { u46 } & & \text { open } & 12 / 21 / 0723: 00\end{array}$

Tubastraea micranthus 10/28/07 6-9m depth near North Point

$\begin{array}{lllr}\text { u32 } & \text { in2, in5, in7 } & & 1 / 25 / 0812: 55 \\ \text { u75 } & \text { out5, out8 } & \text { closed } & 1 / 17 / 0814: 15 \\ \text { u34 } & \text { out5, out8, h } & \text { open } & 1 / 29 / 082: 00 \\ \text { u91 } & & & 12 / 24 / 0713: 00 \\ \text { u97 } & & \text { open } & 12 / 18 / 0723: 30\end{array}$

Tubastraea micranthus 10/28/07 6-9m depth near North Point

$\begin{array}{lllr}\text { u61 } & \text { in2, in5, in7 } & \text { open } & 1 / 28 / 0822: 55 \\ \text { u30 } & \text { out3, out5, out8 } & \text { open } & 1 / 17 / 0821: 10 \\ \text { u7 } & \text { out5, out8, h } & \text { closed } & 1 / 28 / 0815: 25 \\ \text { u103 } & & \text { closed } & 1 / 17 / 0814: 15 \\ \text { u26 } & & & 12 / 22 / 0713: 30\end{array}$

Tubastraea micranthus 10/28/07 6-9m depth near North Point

$\begin{array}{lllr}\text { u31 } & \text { d } & \text { closed } & 1 / 17 / 08 ~ 14: 15 \\ \text { u23-vert } & \text { in4, in5, in7 } & \text { closed } & 1 / 28 / 0816: 45 \\ \text { u63 } & \text { out3, out5, out8 } & \text { open } & 1 / 17 / 0821: 10 \\ \text { u2 } & \text { out5, out8, i } & \text { closed } & 1 / 28 / 0813: 38 \\ \text { u36 } & & \text { open } & 12 / 18 / 0717: 10\end{array}$

Goniastrea edwardsi 10/29/07 near seawater intake

u40 in3, in5, in7 open $1 / 28 / 0822: 30$

u21 out7, out12, out15 closed 1/29/08 2:28

Porites 10/30/07 near seawater intake

$\begin{array}{lllr}\text { u98 } & \text { b } & \text { partly open } & 1 / 28 / 0816: 15 \\ \text { u25 } & \text { g } & \text { partly open } & 1 / 28 / 0815: 30 \\ \text { u38 } & \text { in4, in6 } & & 12 / 28 / 0720: 40 \\ \text { u72 } & \text { out4, out9, out13, out18 } & \text { partly open } & 1 / 29 / 0814: 30 \\ \text { u82 } & \text { out7, out12, out15, out17 } & \text { closed } & 1 / 29 / 082: 28 \\ \text { u22 } & & \text { closed } & 1 / 28 / 0816: 40\end{array}$


Porites 10/30/07 near seawater intake

$\begin{array}{lllr}\text { u80 } & \text { in3, in6, in7 } & \text { partly open } & 1 / 28 / 0822: 50 \\ \text { u50 } & \text { out2, d } & \text { partly open } & 1 / 29 / 0812: 10 \\ \text { u62 } & \text { out5, out8, } f & \text { partly open } & 1 / 28 / 0813: 25 \\ \text { u48 } & \text { out7, out12, out15, out18 } & \text { partly open } & 1 / 29 / 0814: 30 \\ \text { u47 } & & \text { open } & 12 / 18 / 0723: 30\end{array}$

Porites 10/30/07 near seawater intake

\begin{tabular}{|c|c|c|c|}
\hline u59 & $c$ & partly open & $1 / 28 / 0816: 10$ \\
\hline u90 & in2, in6 & & 12/28/07 16:20 \\
\hline u93 & out 4 , out 11 , out 14 , out 17 & open & $1 / 29 / 0814: 40$ \\
\hline 179 & out 4 , out 11 , out 14 , out 18 & partly open & $1 / 28 / 0823: 55$ \\
\hline 99 & & & 12/2/07 23:30 \\
\hline \multicolumn{4}{|c|}{ Goniastrea edwardsi 10/30/07 near seawater intake } \\
\hline 67 & in3, in6, in7 & closed & $1 / 28 / 0815: 40$ \\
\hline 173 & in5, in7 & & $1 / 25 / 0813: 05$ \\
\hline 2 & out 1 , out 5 , out 8, e & partly open & $1 / 28 / 0815: 20$ \\
\hline 11 & out 1 , out 6 , out 10 , out 14 & open & $1 / 29 / 0815: 00$ \\
\hline 10 & & open & 12/21/07 23:00 \\
\hline
\end{tabular}

Goniastrea edwardsi 10/30/07 near seawater intake

$\begin{array}{lllr}\text { u89 } & \text { b } & \text { closed } & 1 / 28 / 0815: 40 \\ \text { u45 } & \text { in2, in5, in7 } & \text { closed } & 1 / 28 / 0823: 15 \\ \text { u39 } & \text { out3, out9, out13 } & \text { partly open } & 1 / 28 / 0823: 10 \\ \text { u54 } & \text { out6, out10, out14 } & \text { open } & 1 / 29 / 082: 38 \\ \text { u85 } & & \text { closed } & 1 / 28 / 0815: 45\end{array}$

Goniastrea edwardsi 10/30/07 near seawater intake

$\begin{array}{lllr}\text { u65 } & \text { c } & \text { closed } & 1 / 28 / 08 ~ 15: 55 \\ \text { u52 } & \text { d } & \text { closed } & 1 / 28 / 08 ~ 23: 30 \\ \text { u13 } & \text { in3 } & \text { partly open } & 12 / 18 / 0723: 30 \\ \text { u86 } & \text { out2, out7, out12, out15 } & \begin{array}{l}\text { partly open } \\ \text { partly open }\end{array} & 1 / 29 / 0814: 00 \\ \text { u3 } & & \begin{array}{l}12 / 16 / 07 ~ 0: 30 \\ \text { partly open }\end{array} & 12 / 18 / 0717: 10 \\ \text { u44 } & & \\ & & \\ \text { Porites } & \text { 10/30/07 near seawater intake } & & 1 / 28 / 0815: 30 \\ \text { u5 } & \text { g } & \text { partly open } & 1 / 25 / 0812: 50 \\ \text { u42 } & \text { in3, in6, in7 } & & 1 / 28 / 0813: 30 \\ \text { u77 } & \text { out1, out5, out8, e } & \text { partly open } & 1 / 29 / 082: 00 \\ \text { u104 } & \text { out18 } & \text { open } & 1 / 29 / 0814: 45\end{array}$


Goniastrea edwardsi 10/30/07 near seawater intake

$\begin{array}{ll}\text { u43 } & \text { in2, in6, in7 } \\ \text { u96 } & \text { out2 } \\ \text { u19 } & \text { out5, f, out8 } \\ \text { u102 } & \text { out7, out12, out15, } \\ \text { u37 } & \\ \text { u78 } & \\ \text { Millepora } & \\ \text { u33 } & \text { in3, in5, in7 } \\ \text { u53 } & \text { out4, out9, e } \\ \text { u4 } & \text { out7, out12, f } \\ \text { u14 } & \\ \text { u18 } & \end{array}$

$\begin{array}{lr}\text { open } & 1 / 28 / 0823: 20 \\ \text { open } & 1 / 17 / 0821: 30 \\ \text { open } & 1 / 28 / 0813: 00 \\ \text { open } & 1 / 29 / 0813: 45 \\ & 12 / 15 / 0715: 30 \\ & 12 / 22 / 0713: 30\end{array}$

1/28/09 23:25

$1 / 28 / 0813: 43$

$1 / 28 / 0823: 45$

12/12/07 17:00

12/15/07 15:30 

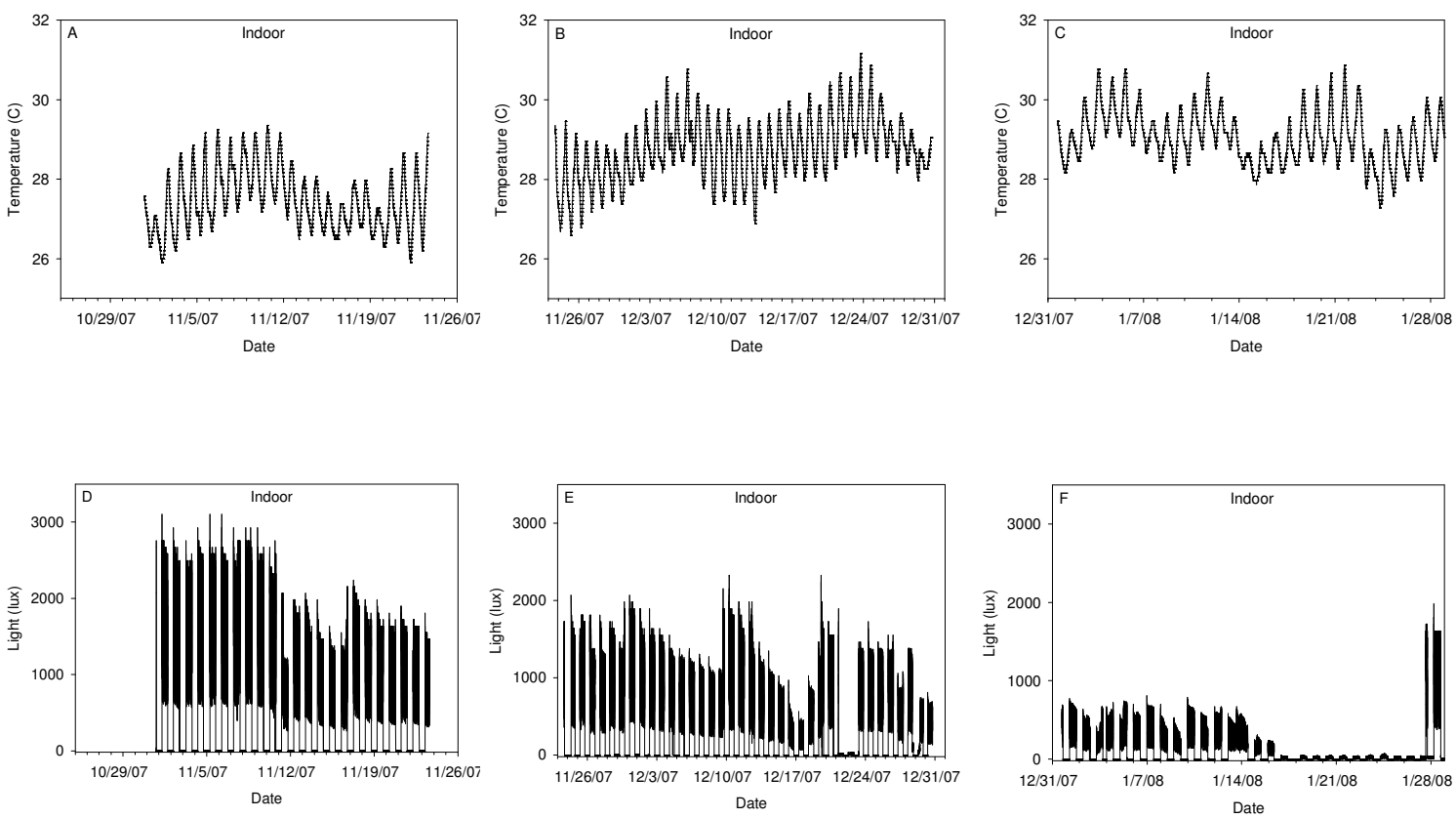

Figure A-1. Light and temperature logs for the indoor aquarium 

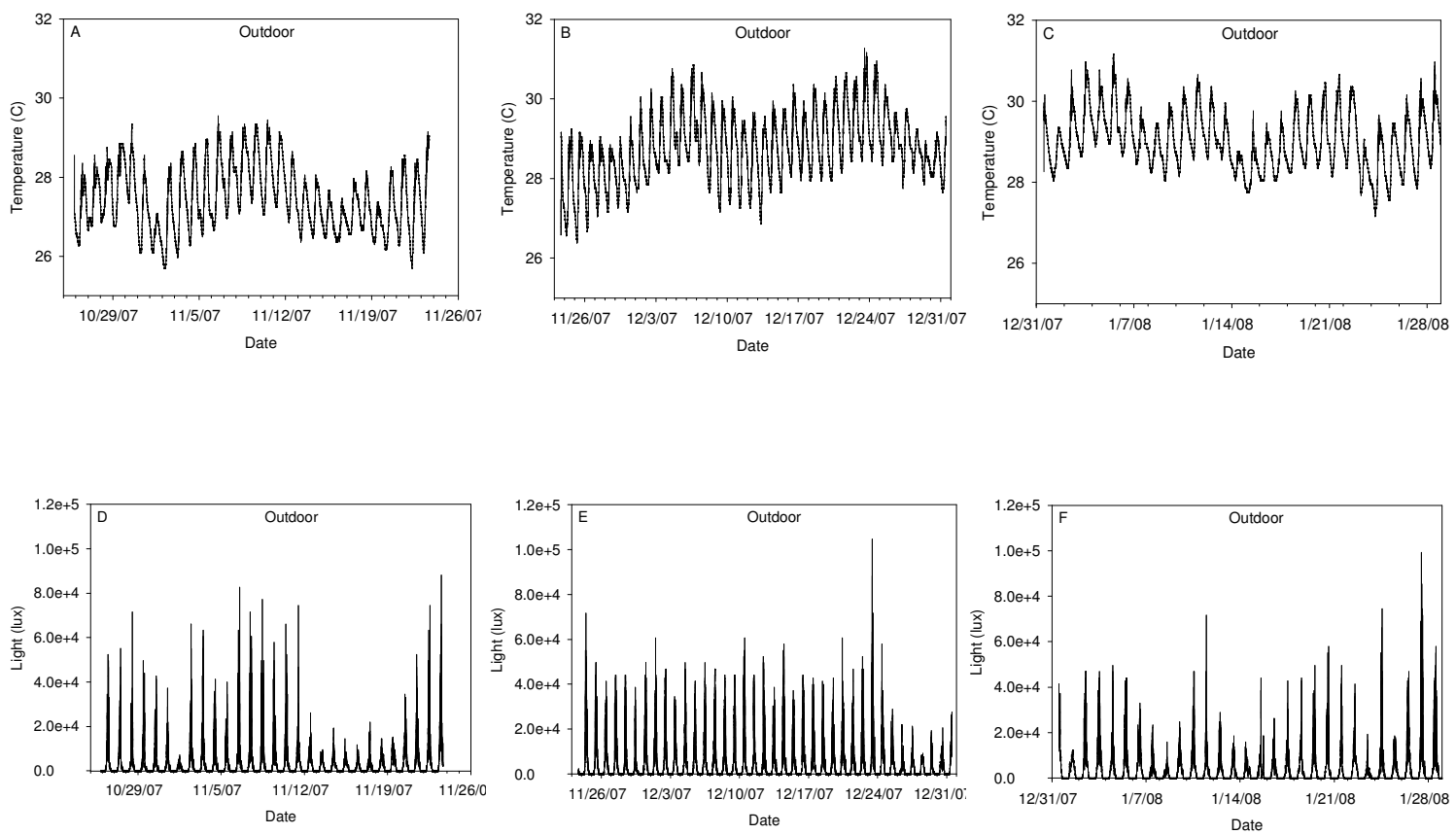

Figure A-2. Light and temperature logs for the Outdoor tank. 

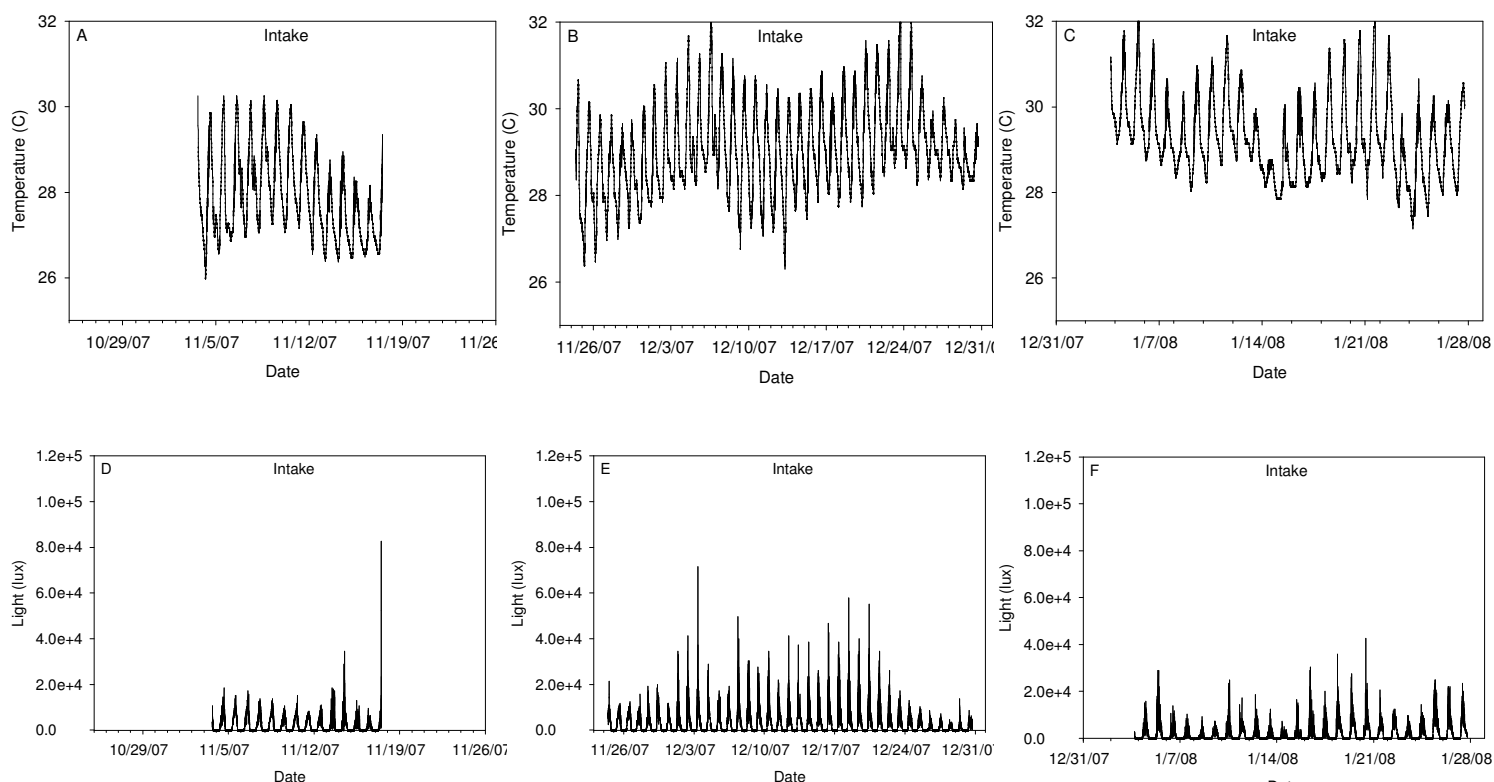

Figure A-3. Light and temperature logs for the intake. 


\section{Appendix 3: Supplemental data for Chapter 3}

In addition to the experiments described in Chapter 3, an additional component - one water bath held at $19^{\circ} \mathrm{C}$ was also part of the experiment. Each treatment included three replicate corals/containers, however treatments for two containers were switched part way through the experiment, thus insufficient replicates exist for statistical comparisons. Experiments were continued for an additional 6 weeks beyond the data presented, however nutrient levels rose during this time, so treatments were no longer comparable and thus are not presented. Both temperatures are included in Fig. A-1 for comparison with similar data presented in the Chapter 4 appendix (Fig. A-13). 

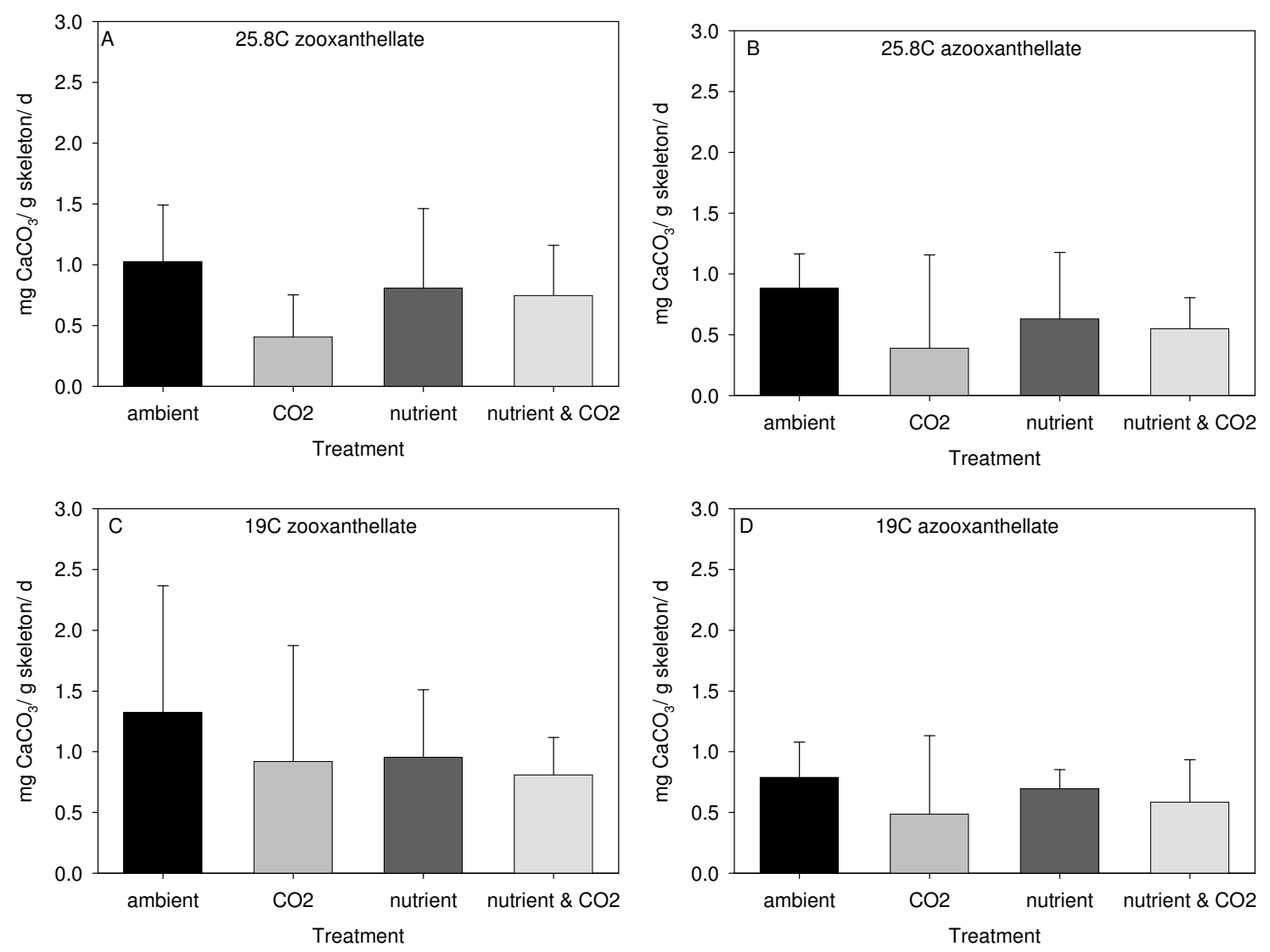

Figure A-1. Mass normalized growth rates for each treatment used in Chapter 3. Data are expressed as $\mathrm{mg} \mathrm{CaCO}_{3}$ deposited per day during the treatment phase per $\mathrm{g}$ of final skeletal dry weight. A. Growth rates for zooxanthellate corals maintained at $25.8^{\circ} \mathrm{C}$. B. Growth rates for originally azooxanthellate corals maintained at $25.8^{\circ} \mathrm{C}$. C. Growth rates for zooxanthellate corals maintained at $19^{\circ} \mathrm{C}$. D. Growth rates for originally azooxanthellate corals maintained at $19^{\circ} \mathrm{C}$. Bars represent means, error bars are standard deviation. Note: azooxanthellate colonies turned brown over the course of the experiment - data are included for comparison purposes with Chapter 4. 
Appendix A4: Chapter 4 appendix. Additional methods, results, aquarium conditions (Table A1 and Fig. A- 1), and physiological parameters measured on corals (Fig. A- 2$13)$.

\section{Additional methods}

Chemistry

All vials used for water sampling had polypropylene lids with foamed polyethylene liners. Vials and lids were soaked in distilled water, rinsed repeatedly and dried prior to use. Vials for cation samples were soaked in $10 \% \mathrm{HCl}$ prior to washing with distilled water.

\section{Alkalinity}

Alkalinity samples were taken in glass or plastic scintillation vials (Wheaton). Samples were stored refrigerated for no more than 1 month prior to measurement. Alkalinities were measured via titration with $0.01 \mathrm{~N} \mathrm{HCl}$ containing $40.7 \mathrm{~g} \mathrm{NaCl} / \mathrm{L}$ using a Metrohm Titrando 808 dosimat and 730 Sample Changer controlled by Tiamo software to perform automated normalized gran titrations of $1 \mathrm{ml}$ samples. Replicate samples were run and additional replicates run if measured values differed by more than $4 \mu \mathrm{mol} / \mathrm{kg}$. Certified seawater reference material supplied by the lab of Andrew Dickson (Scripps Institution of Oceanography) was run each time samples were run to determine acid normality. Monitoring data is presented in Figure A-1h.

$p H$ 
Prior to the treatment phase, $14 \mathrm{ml} \mathrm{pH}$ samples were collected in $15 \mathrm{ml}$ polypropylene conical tubes (Corning) which had previously been washed repeatedly with fresh water and soaked repeatedly with seawater (to leach out acidic compounds in the plastic). Thymol blue (Fisher lot\#990191) was added to each sample (160 or $320 \mu \mathrm{l}$ of a $2.05 \mathrm{mmol} / \mathrm{L}$ solution). Samples were allowed to temperature equilibrate in a water bath prior to transfer to a $1 \mathrm{~cm}$ cuvette for absorbance measurements. Spectra were collected using an Ocean Optics USB4000 spectrophotometer with a blue filtered tungsten light source (Ocean Optics LS-1-LL). For each sample, 150 spectra were averaged, seawater from one of the sampled tanks was used for establishing reference absorbance. $\mathrm{pH}$ (total scale) was calculated following Zhang and Byrne (1996) including the correction of Delvalls and Dickson (1998).

During the treatment phase, $\mathrm{pH}$ values in the $\mathrm{CO}_{2}$ treatments were too low to be measured with thymol blue, thus m-cresol purple (Acros 199250050, lot A014923001) was used instead. Initial m-cresol purple measurements were made as described above except dye additions were 50 or $100 \mu \mathrm{l}$ of a $9.4 \mathrm{mmol} / \mathrm{L}$ solution, however most were made using a $10 \mathrm{~cm}$ path length flow cell instead of a cuvette. For measurements with the flow cell, samples were collected in pre-cleaned $10 \mathrm{ml}$ polypropylene syringes (Becton Dickson), a small air bubble introduced (for mixing) along with 50 or $100 \mu \mathrm{l}$ of a $0.946 \mathrm{mmol} / \mathrm{L}$ solution of $\mathrm{m}$-cresol purple. Syringes were capped and placed in a constant temperature bath prior to injection into the flow cell. $\mathrm{pH}$ calculations followed DOE (1994). Values are plotted in Fig. A-1i. 
In addition to spectrophotometric measurements, $\mathrm{pH}$ (NBS scale) measurements were also made using an Orion Ross 8165BNWP electrode calibrated with NBS buffers (Ricca) and a pH meter (Orion). For some measurements, the $\mathrm{pH}$ electrode was placed directly in the container to be measured. For monitoring day/night trends in aquarium $\mathrm{pH}$, a siphon tube was inserted into the aquarium to be measured through the hole used for feeding. Water siphoned from the aquarium flowed through a small vial holding the electrode and a temperature probe. $\mathrm{pH}$ values are plotted in Figure $\mathrm{A}-1 \mathrm{j}$.

\section{$\delta^{8} \mathrm{O} \mathrm{H}_{2} \mathrm{O}$ and $\mathrm{DIC}$}

Samples for $\delta^{18} \mathrm{O} \mathrm{H}_{2} \mathrm{O}$ and $\delta^{13} \mathrm{C}$ DIC were collected in glass scintillation vials. For $\delta^{18} \mathrm{O} \mathrm{H}_{2} \mathrm{O}$, vials were pre-weighed, filled to near the top using a syringe, capped and then re-weighed. Vials were stored at room temperature. Prior to being sent for analysis, all vials were re-weighed to estimate evaporation rates and then sent to the laboratory of Dan Schrag (Harvard University) for $\delta^{18} \mathrm{O} \mathrm{H}_{2} \mathrm{O}$ analysis (Fig. A-11). DIC vials were filled to near the top of the vial, $10 \mu \mathrm{l}$ saturated $\mathrm{HgCl}_{2}$ added, and kept refrigerated until an aliquot could be sealed in glass ampoules. DIC measurements (manometer based) and $\mathrm{CO}_{2}$ extractions for $\delta^{13} \mathrm{C}$ DIC analysis were done by R. Belastock, $\delta^{13} \mathrm{C}$ measurements were made by NOSAMS (WHOI) (Fig. A-1m,n).

\section{Cations}

Cation samples were taken in pre-weighed HDPE vials. Each sample was filtered through a $0.45 \mu \mathrm{m}$ filter which had first been rinsed with at least $20 \mathrm{ml}$ of sample. 
Sample sizes were $\sim 20 \mathrm{ml}$. Each vial was weighed and then stored at room temperature (no measurements were made).

\section{Salinity}

Salinity was monitored using a conductivity probe (Hach) (Fig. A- 1k). Probe stability was checked periodically using 20 and $100 \mathrm{mS} \mathrm{NaCl}$ conductivity standards

(Ricca). Conductivity was converted to salinity values using equations given by Fofonoff (1985). From the reservoirs supplying the water for each of the aquaria, occasional samples were taken in $125 \mathrm{ml}$ glass vials with screw cap lids containing conical plastic seals for salinity measurement on a Guildline autosal model 8400B salinometer.

\section{Additional physiological parameters}

\section{Alkalinity depletion rates}

Before starting treatments and again after treatment levels for $\mathrm{CO}_{2}$ and nutrients had been reached, day and night growth rates for each coral were measured via alkalinity depletion. Pre-washed (with fresh and seawater) 1L PET food service containers with lids (SOLO) were weighed to $0.01 \mathrm{~g}, \sim 500 \mathrm{ml}$ of water with the same chemistry as being used for treatments was added to each, containers re-weighed, a coral, blank slide, or nothing was added to each, containers re-weighed and placed into a constant temperature bath. Airstones were added to each container and each container bubbled continuously with the appropriate treatment gas throughout the incubation. A syringe was used to collect initial alkalinity samples from each container and the amount of water removed 
was weighed. After 8-11 hr a second sample was taken, the lighting condition changed (lights on or off) and corals maintained for another 8-11 hr. Light levels (PAR) ranged from $20-30 \mu \mathrm{mol}$ photons $/ \mathrm{m}^{2} / \mathrm{s}$. At the end of the incubation, containers were removed from the bath, airstones removed, containers dried and weighed. Alkalinity samples were taken from each container, $\mathrm{pH}$ (electrode) and salinity measured (conductivity probe), and corals returned to their tanks. Aragonite deposition was assumed to be the only process affecting alkalinity (this under-estimates calcification due to ammonia release by the coral, preliminary data suggests up to a $15 \%$ underestimation), with 2 mol alkalinity consumed per mol of $\mathrm{CaCO}_{3}$ deposited. Alkalinity depletion rates were corrected for evaporation (based on the change in container mass, rates were assumed to be linear), and for background rates measured in containers without corals. Background alkalinity consumption rates were invariably low relative to coral rates.

\section{Oxygen consumption}

To measure oxygen, a luminescence-based dissolved oxygen probe (Hach) was used. Probe stability was assessed every few days by measuring nitrogen and compressed air. For oxygen consumption/evolution incubations, small aquarium pumps (AZOO) were sealed inside 1 L PET containers using Aquamend epoxy and silicone sealant. All unnecessary plastic components were removed from each of the pumps to reduce spaces for trapping air bubbles, flow rates were reduced to $\sim 90 \mathrm{~L} / \mathrm{h}$ by trimming the impeller. A PET tray was used to hold corals $\sim 2 \mathrm{~cm}$ off the bottom of the container, water flow from the pump passed through one hole in the tray, and several holes allowed 
water to return to the pump. Recessed PET lids were used for sealing the containers and the contact area between the container and lid was coated with silicone grease to exclude air. Total volume was $\sim 930 \mathrm{ml}$. Containers were filled to overflowing with treatment water, the pump started and stopped repeatedly and a pipet used to dislodge air bubbles. All corals/slides were carefully cleaned of algae, however algal growth on the cyanoacrylate surrounding the corals and on the control slides could not be effectively removed due to the roughness of the surface. A coral, blank slide, or nothing was added to each container, the container sealed, placed in a water bath and the pump started. Each container was opened $\sim 6 \mathrm{hr}$ later, $\mathrm{pH}$ (electrode), conductivity, temperature and oxygen measured. Samples for alkalinity and nutrient analysis were collected. Containers were then left open for $\sim 1 \mathrm{hr}, 90 \mathrm{ml}$ of treatment water added, bubbled with the appropriate gas, lighting state changed, and final $\mathrm{pH}$, conductivity, temperature, and oxygen partial pressure measured. Atmospheric pressure was recorded at the time of each set of measurements. Containers were sealed, incubated for another $\sim 6 \mathrm{hr}$, opened and sampled again prior to returning corals to the appropriate tank. Light levels were similar to those used for alkalinity depletion incubations. Oxygen concentrations at 1 atm were calculated using equation 8 of Garcia and Gordon (1992) and coefficients for Benson and Krause (1984), the first $\mathrm{A}_{3}$ term listed in equation 8 was not included in the calculation; oxygen concentration was assumed to scale linearly with atmospheric pressure. Background rates of oxygen production by control slides could account for up to $100 \%$ of the apparent oxygen production by corals, oxygen consumption by control slides could account for up to $50 \%$ of the total oxygen consumption. Coral rates were corrected for 
the average background consumption/production rate measured at the same time; however, such high background rates limit the utility of the data, especially for zooxanthellate corals.

\section{Photosynthetic parameters}

A diving pulse amplitude modulation fluorimeter (diving PAM, WALZ) was used to measure minimum fluorescence values $\left(\mathrm{F}_{\mathrm{o}}\right)$ and maximal fluorescence $\left(\mathrm{F}_{\mathrm{m}}\right)$ in the dark and light adapted minimum $(\mathrm{F})$ and maximum $\left(\mathrm{F}_{\mathrm{m}}\right)$ fluorescence and to perform rapid light curves. PAM settings were: measurement intensity 4, damping 2, gain 2, saturating intensity 6 , saturating width $0.6 \mathrm{~s}$, light curve initial value 1 , amplification 1 , light curve width 0:10 s. For all measurements, an acrylic holder was used to hold the end of the PAM fiber optics $3.6 \mathrm{~cm}$ from the surface of the slide. One side of the holder was opaque to block excitation light from reaching corals that had not been measured. Light adapted yield $\left(\Delta \mathrm{F} / \mathrm{F}_{\mathrm{m}}\right)$ measurements were performed at 3 spots across each coral during the late afternoon to early evening. Rapid light curves and dark adapted yields $\left(\mathrm{F}_{\mathrm{v}} / \mathrm{F}_{\mathrm{m}}\right)$ were performed at two spots on each coral (on different nights with the coral in a different orientation relative to the probe each night) starting at least $2.5 \mathrm{~h}$ after dark. Each spot used was the one with the highest fluorescence yield $\left(F_{0}\right)$ found with a brief search. A red LED bulb was used to provide light during positioning of the holder over each coral. The distance between the tip of the fiber optics and the coral surface varied, however the coral height has not been measured, thus PAR values measured at a typical coral distance $(1.5 \mathrm{~cm})$ using the PAM PAR sensor have been used for calculating parameters from 
rapid light curves. Relative electron transport rates (rETR) were calculated as follows: $\mathrm{rETR}=\Delta \mathrm{F} / \mathrm{F}_{\mathrm{m}}, \mathrm{x}$ PAR. PAR and rETR data were fit to the model of Platt et al. (1980) using Matlab to calculate values for the initial slope $(\alpha)$, photo-inhibition $(\beta)$, maximum relative electron transport rate $\left(\mathrm{rETR}_{\max }\right)$ saturating light intensity $\left(\mathrm{I}_{\mathrm{k}}\right)$, and irradiance at maximal rETR $\left(\mathrm{I}_{\mathrm{m}}\right)$. Measurements on azooxanthellate corals gave fluorescence values near zero and are not analyzed.

\section{Tissue Sampling}

Fragments for tissue sampling were placed in plastic bags with $5 \mathrm{ml}$ of cold 0.02 $\mu \mathrm{m}$ filtered seawater. Tissue was airbrushed from the skeleton using compressed air and a pipet tip to focus the air. The tissue slurry was homogenized briefly with a tissue grinder (Kontes). Samples were transferred to $15 \mathrm{ml}$ polypropylene conical tubes and centrifuged for $1 \mathrm{~min}$ at $4^{\circ} \mathrm{C}$ at a setting of 1 on an IEC Clinical Centrifuge with swinging bucket rotor to settle skeletal debris. An aliquot of the supernatant was transferred to a $1.5 \mathrm{ml}$ polypropylene tube and placed at $-80^{\circ} \mathrm{C}$. The remaining supernatant was centrifuged for 2 min at a setting of 2 . The supernatant was drawn off and placed at $80^{\circ} \mathrm{C}$, the pellet was resuspended in $0.5 \mathrm{ml}$ of filtered seawater and frozen. All samples are stored at $-80^{\circ} \mathrm{C}$ for future analysis. Tissue sampling will be repeated at the completion of the experiment.

\section{Photography}


Prior to taking buoyant weights, every coral was photographed. Photographs were taken using a Canon digital rebel XTi camera with macro lens hooked up to a computer running DSLR Remote pro software (Breeze Systems). The camera was placed at a constant distance from the side of an acrylic tank. Lighting was provided by 2 white LED bulbs attached to the front of the tank on either side of the camera lens. Corals were placed on a stand at the back of the tank, slides were aligned with the sides of the stand to allow positions to be reproduced. Six side view images were taken, four as stereo pairs. Six top view images were taken, four as stereo pairs. Over the course of the experiment corals overgrew their slides preventing alignment of slides with the stand (without risking damage to the coral), photographs were still taken, but not at precise orientations. Images provide documentation of growth, pigmentation, and tissue cover, which may be of interest for future analysis.

\section{Statistics}

Treatments $\left(\mathrm{CO}_{2}\right.$ and nutrient) were compared using a factorial ANOVA model, including the gender and the parent colony within gender as a blocking variable, each temperature and symbiotic state was analyzed separately. For ANOVA analysis, all data were expressed as differences between the treatment and pre-treatment rates relative to the pre-treatment rate (except for oxygen consumption and PAM data, which were analyzed as differences). ANOVAs were run using SAS, linear regressions were performed using Systat. All data are given as mean with standard deviation in parenthesis unless otherwise noted. 


\section{Results}

\section{Aquarium conditions}

Flow rates varied on a daily basis (Fig. A- 1a), with an average flow of 8 (1.5) $\mathrm{L} / \mathrm{h}$, which corresponds to a flow rate of $\sim 25 \mathrm{ml} / \mathrm{min}$ for $16^{\circ} \mathrm{C}$ tanks and $\sim 40 \mathrm{ml} / \mathrm{min}$ for $24^{\circ} \mathrm{C}$ tanks. Temperature was stable in the $24^{\circ} \mathrm{C}$ treatment, while it rose by $0.3^{\circ} \mathrm{C}$ during the summer for the $16^{\circ} \mathrm{C}$ bath (Fig. A- 1b). Water chemistry in each tank closely followed the nutrient and alkalinity chemistry in the source reservoir (Fig. A- 1c-f,h), suggesting adequate flow rates were usually maintained. Ammonia levels were consistently elevated inside the aquaria relative to the source water, likely as a result of coral metabolism (Fig. A- 1c). Silicate was generally lower in the aquaria relative to source water, likely as a result of diatom growth within the tanks (Fig. A- 1d). Phosphate (Fig. A-1e) and nitrate (Fig. A-1f) closely followed the source water chemistry. Alkalinity values within tanks are consistently below those of the source water, likely as a result of calcification. Substantial deviations from the source chemistry are seen in both the nutrient and alkalinity data, these tend to be associated with declines in flow rate to an individual tank and likely represent the maximum departure from source values experienced by the corals. Nutrient levels rose substantially during the summer in the ambient seawater (Fig. A- 1e,f), while alkalinity declined (Fig. A- 1h). The partial pressure of $\mathrm{CO}_{2}$ (Fig. A- 1g) in ambient air showed a small decline during the summer, with an average of 375(14) $\mu \mathrm{atm}$ (minimum 340, maximum 410). The $\mathrm{pCO}_{2}$ for the $\mathrm{CO}_{2}$ 
treatment showed greater variability due to periodic readjustment of the rotameter, averaging 795(26) $\mu \mathrm{atm}$ (minimum 740, maximum 850). Atmospheric pressure displayed no consistent trends (Fig. A- 1g). pH showed diurnal cycles with lower values at night and higher values during the day, however consistent offsets between $\mathrm{CO}_{2}$ treatments were maintained and daily ranges were smaller than differences between $\mathrm{CO}_{2}$ treatments (Fig. A- 1i,j) $d^{18} \mathrm{O}$ of the water remained relatively stable throughout the experiment, averaging -1.21(0.06) per mil (Fig. A- 11). Oxygen saturation was measured occasionally in each aquarium, values ranged from $95 \%$ to $102 \%$ of saturation (data not shown).

\section{Growth rates}

\section{Alkalinity depletion}

Figures 4 and 5 show rates of alkalinity uptake for light and dark alkalinity runs (Fig. A-4) and oxygen incubations (Fig. A- 5). Rates of alkalinity uptake are generally lower at $16^{\circ} \mathrm{C}$ than at $24^{\circ} \mathrm{C}$. Significant treatment effects $(\mathrm{p}<0.001)$ were found for $\mathrm{CO}_{2}$ in oxygen incubations of zooxanthellate corals at $24^{\circ} \mathrm{C}$ in the light, gender and individual parent colony were also significant $(\mathrm{p}<0.05)$. A significant $(\mathrm{p}<0.05) \mathrm{CO}_{2}$ and nutrient interaction was found for azooxanthellate corals at $16^{\circ} \mathrm{C}$ in the light for oxygen incubations, parent colony was also significant $(\mathrm{p}<0.05)$. Differences among treatments are all non-significant for alkalinity runs, however, consistent with buoyant weight data, males tend to calcify at higher rates than females (Fig. A- 4d-g, 5d-g).

Alkalinity depletion $v$ buoyant weight 
Coral calcification rates were measured via buoyant weight and via alkalinity depletion measurements. Alkalinity depletion measurements were made on two different types of incubations, one in which corals were actively bubbled throughout the incubation (alkalinity runs), the other in which corals were sealed in containers and water circulated without gas exchange $\left(\mathrm{O}_{2}\right.$ incubations). Buoyant weight calcification rates were compared with averaged (day and night) alkalinity depletion rates measured during the same time interval (Fig. A- 2a). Rates were significantly correlated $(\mathrm{p}<0.0001)$ and described by the linear regression equation: alkalinity depletion rate $(\mathrm{mg} / \mathrm{d})=$ $0.82 *$ buoyant weight rate $(\mathrm{mg} / \mathrm{d})+0.22\left(\mathrm{R}^{2}=0.76\right)$. Calcification rates measured during $\mathrm{O}_{2}$ incubations and alkalinity runs (Fig. A- 2b) were significantly correlated ( $<<0.0001$ ) and described by the equation: $\mathrm{O}_{2}$ incubation rate $=1.06 *$ alkalinity run rate +0.63 $\left(\mathrm{R}^{2}=0.54\right)$.

As shown previously (Holcomb et al. submitted), buoyant weight and alkalinity depletion measurements are significantly correlated (Fig. A- 2a). However, alkalinity depletion rates are consistently lower than rates measured via buoyant weights. Reduced alkalinity depletion rates are probably a combination of ammonia production by the coral, and a reduction in the alkalinity over the course of the incubation which can slow growth. Growth rates measured via alkalinity depletion in alkalinity runs were systematically lower than rates measured in oxygen incubations (Fig. A- 2b). Such differences may be the result of higher flow rates in oxygen incubations, however, the shorter incubation times and larger incubation volume used in oxygen incubations led to reduced alkalinity changes and less ammonia build up, which would be expected to increase measured rates. 
The relatively poor correlation between alkalinity uptake rates measured in oxygen incubations and alkalinity runs may result from the small alkalinity changes measured in oxygen incubations, such that analytical error in alkalinity determinations could amount to over $20 \%$ of the total alkalinity change.

\section{Oxygen production/consumption}

Net oxygen consumption/production rates were measured in the light and dark (Fig. A- 6). Zooxanthellate corals showed lower rates of oxygen consumption in the light, and at $16^{\circ} \mathrm{C}$, oxygen production exceeded consumption for most treatments. At $24^{\circ} \mathrm{C}$ in the light, zooxanthellate corals showed significant effects for nutrients $(\mathrm{p}<0.01)$ and gender $(\mathrm{p}<0.05)$. Nutrient enriched treatments tended to have lower oxygen consumption rates, as female corals. In the dark, gender $(\mathrm{p}<0.05)$ and $\mathrm{CO}_{2}(\mathrm{p}<0.01)$ were both significant, with male corals having higher respiration rates and $\mathrm{CO}_{2}$ treatments having higher respiration rates. At $24^{\circ} \mathrm{C}$ in the dark and in the light, azooxanthellate corals showed a significant $(\mathrm{p}<0.01) \mathrm{CO}_{2}$ nutrient interaction and a gender effect. Female corals tended to have lower respiration rates. At $16^{\circ} \mathrm{C}$, only azooxanthellate corals in the dark had a significant ANOVA showing a $\mathrm{CO}_{2}$ effect $(\mathrm{p}<0.05)$.

PAM

PAM data show no clear trends with respect to treatment variables (Fig. A- 7-12). A significant $(\mathrm{p}<0.01)$ interaction between $\mathrm{CO}_{2}$ and nutrients was found for light adapted yields (Fig. A- 9a,b) at both $16^{\circ} \mathrm{C}$ and $24^{\circ} \mathrm{C}$. At $16^{\circ} \mathrm{C}$, yields decreased relative to initial 
for both nutrient and $\mathrm{CO}_{2}$ treatments, while yields increased with the combined treatment. At $24^{\circ} \mathrm{C}$ at the start of treatment, yields increased for both nutrient and $\mathrm{CO}_{2}$ treatments, while yields decreased for the combined treatment. All other parameters derived from PAM data showed no significant treatment effects. 
Table A-1. PAR ( $\mu \mathrm{mol}$ photons $\left./ \mathrm{m}^{2} / \mathrm{s}\right)$ received by each treatment at both the surface of the aquarium and at $1.5 \mathrm{~cm}$ above the bottom of the aquarium (coral depth) as measured with the diving-PAM fiber optic quantum sensor (Walz).

\begin{tabular}{|l|l|l|}
\hline Treatment & PAR (tank surface) & PAR (coral depth) \\
\hline $24^{\circ} \mathrm{C}$ ambient & 80 & 17 \\
\hline $24^{\circ} \mathrm{C}$ nutrient & 90 & 20 \\
\hline $24^{\circ} \mathrm{C} \mathrm{CO}_{2}$ & 90 & 20 \\
\hline $24^{\circ} \mathrm{C}$ nutrient \& $\mathrm{CO}_{2}$ & 85 & 19 \\
\hline $16^{\circ} \mathrm{C}$ ambient & 90 & 20 \\
\hline $16^{\circ} \mathrm{C}$ nutrient & 70 & 15 \\
\hline $16^{\circ} \mathrm{C} \mathrm{CO} 2$ & 80 & 18 \\
\hline $16^{\circ} \mathrm{C}$ nutrient $\& \mathrm{CO}_{2}$ & 50 & 12 \\
\hline
\end{tabular}


Table A-2. ANOVA results for each temperature/zooxanthellae combination testing for effects of nutrients, $\mathrm{CO}_{2}$ and the interaction thereof.

\begin{tabular}{|c|c|c|c|c|c|c|}
\hline T/zoox & Source & DF & SS & Mean & $\mathrm{F}$ & $p$ \\
\hline \multicolumn{7}{|c|}{ 24C zooxanthellate } \\
\hline & Model & 6 & 3.317 & 0.553 & 5.32 & 0.0057 \\
\hline & Error & 13 & 1.351 & 0.104 & & \\
\hline & & & & & & \\
\hline & gender & 1 & 2.061 & 2.061 & 19.83 & 0.0007 \\
\hline & corals(gender) & 2 & 0.198 & 0.099 & 0.95 & 0.4106 \\
\hline & $\mathrm{co} 2$ & 1 & 0.932 & 0.932 & 8.96 & 0.0104 \\
\hline & nut & 1 & 0.122 & 0.122 & 1.18 & 0.2977 \\
\hline & nut*co2 & 1 & 0.003 & 0.003 & 0.03 & 0.8655 \\
\hline \multicolumn{7}{|c|}{ 24C azooxanthellate } \\
\hline & Model & 6 & 5.276 & 0.879 & 4.57 & 0.0105 \\
\hline & Error & 13 & 2.504 & 0.193 & & \\
\hline & & & & & & \\
\hline & gender & 1 & 4.358 & 4.358 & 22.63 & 0.0004 \\
\hline & corals(gender) & 2 & 0.188 & 0.094 & 0.49 & 0.6247 \\
\hline & co2 & 1 & 0.030 & 0.030 & 0.16 & 0.6977 \\
\hline & nut & 1 & 0.689 & 0.689 & 3.58 & 0.081 \\
\hline & nut $^{*}$ co2 & 1 & 0.010 & 0.010 & 0.05 & 0.8193 \\
\hline \multicolumn{7}{|c|}{ 16C zooxanthellate } \\
\hline & Model & 6 & 0.967 & 0.161 & 2.59 & 0.0961 \\
\hline & Error & 9 & 0.559 & 0.062 & & \\
\hline & aender & 1 & 0497 & 0497 & 801 & 00197 \\
\hline & corals(gender) & 2 & 0.192 & 0.096 & 1.54 & 0.2651 \\
\hline & $\mathrm{co} 2$ & 1 & 0.203 & 0.203 & 3.27 & 0.1042 \\
\hline & nut & 1 & 0.072 & 0.072 & 1.15 & 0.3108 \\
\hline & nut $^{*}$ co2 & 1 & 0.003 & 0.003 & 0.05 & 0.8246 \\
\hline \multicolumn{7}{|c|}{ 16C azooxanthellate } \\
\hline & Model & 6 & 2.569 & 0.428 & 4.47 & 0.0225 \\
\hline & Error & 9 & 0.862 & 0.096 & & \\
\hline & & & & & & \\
\hline & gender & 1 & 1.493 & 1.493 & 15.6 & 0.0034 \\
\hline & corals(gender) & 2 & 0.626 & 0.313 & 3.27 & 0.0857 \\
\hline & $\operatorname{co2}$ & 1 & 0.379 & 0.379 & 3.96 & 0.0778 \\
\hline & nut & 1 & 0.000 & 0.000 & 0 & 0.9693 \\
\hline & nut*co2 & 1 & 0.071 & 0.071 & 0.74 & 0.4124 \\
\hline
\end{tabular}


Table A-2 continued. ANOVA results for each temperature testing for effects of gender, $\mathrm{CO}_{2}$ and the interaction thereof (nutrient levels have been pooled).

\begin{tabular}{|c|c|c|c|c|c|c|}
\hline $\mathrm{T}$ & Source & DF & SS & $\begin{array}{l}\text { Mean } \\
\text { square }\end{array}$ & $\mathrm{F}$ & \multirow[t]{2}{*}{$p$} \\
\hline \multicolumn{6}{|c|}{ 24C pooling nutrients, zooxanthellate and azooxanthellate } & \\
\hline & Model & 9 & 8.880 & 0.987 & 7.11 & $<.0001$ \\
\hline & Error & 30 & 4.166 & 0.139 & & \\
\hline & & & & & & \\
\hline & $\mathrm{co} 2$ & 1 & 1.552 & 1.552 & 11.18 & 0.0022 \\
\hline & gender & 1 & 6.097 & 6.097 & 43.91 & $<.0001$ \\
\hline & $\begin{array}{l}\text { corals(gend } \\
\text { er) }\end{array}$ & 6 & 0.395 & 0.066 & 0.47 & 0.8217 \\
\hline & gender ${ }^{*} \mathrm{co} 2$ & 1 & 0.835 & 0.835 & 6.01 & 0.0202 \\
\hline \multicolumn{6}{|c|}{ 16C pooling nutrients, zooxanthellate and azooxanthellate } & \\
\hline & Model & 9 & 3.793 & 0.421 & 5.88 & 0.0003 \\
\hline & Error & 22 & 1.576 & 0.072 & & \\
\hline & & & 0 & $0-70$ & 705 & 001 \\
\hline & gender & 1 & 0.580 & $0.5 / 0$ & $\frac{1.95}{1.11}$ & 0.3027 \\
\hline & $\begin{array}{l}\text { corals(gend } \\
\text { er) }\end{array}$ & 6 & 3.128 & 0.521 & 7.27 & 0.0002 \\
\hline & gender ${ }^{*} \mathrm{co} 2$ & 1 & 0.016 & 0.016 & 0.22 & 0.6462 \\
\hline
\end{tabular}


Table A-3. Spawning observations. All coral fragments observed spawning are listed along with the parent colony from which the given fragment was derived (parent), whether or not the parent had zooxanthellae ( $\mathrm{n}=$ azooxanthellate, $\mathrm{y}=$ zooxanthellate), the treatment to which the coral had been exposed prior to the spawning observation (observations during the initial phase are indicated by pre-), whether the coral was observed to spawn as a male or a female, date of observation, and whether observations were made during buoyant weighing (bw) or alkalinity depletion (alk) incubations. All fragments listed were grown at $24^{\circ} \mathrm{C}$.

\begin{tabular}{|c|c|c|c|c|c|c|}
\hline zooxanthellae & parent & fragment & treatment & gender & date & observ. \\
\hline $\mathrm{n}$ & 1 & w32 & nutrient \&CO2 & $\mathrm{m}$ & $11 / 22 / 09$ & bw \\
\hline $\mathrm{n}$ & 1 & w39 & pre-CO2 & $\mathrm{m}$ & $6 / 12 / 09$ & bw \\
\hline $\mathrm{n}$ & 1 & w39 & $\mathrm{CO} 2$ & $\mathrm{~m}$ & 9/20/09 & bw \\
\hline $\mathrm{n}$ & 1 & w48 & ambient & $\mathrm{m}$ & $7 / 4 / 09$ & bw \\
\hline $\mathrm{n}$ & 1 & w85 & pre-ambient & $\mathrm{m}$ & $11 / 14 / 08$ & bw \\
\hline $\mathrm{n}$ & 1 & w85 & pre-ambient & $\mathrm{m}$ & $6 / 12 / 09$ & bw \\
\hline $\mathrm{n}$ & 1 & w85 & ambient & $\mathrm{m}$ & $7 / 4 / 09$ & bw \\
\hline $\mathrm{n}$ & 2 & w18 & pre-nutrient \& CO2 & $f$ & $6 / 12 / 09$ & bw \\
\hline $\mathrm{n}$ & 2 & w20 & pre-ambient & $\mathrm{f}$ & $11 / 14 / 08$ & bw \\
\hline $\mathrm{n}$ & 3 & w10 & pre-nutrient \& CO2 & $f$ & $6 / 12 / 09$ & bw \\
\hline $\mathrm{n}$ & 3 & w37 & $\mathrm{CO} 2$ & $f$ & 9/20/09 & bw \\
\hline $\mathrm{n}$ & 3 & w37 & $\mathrm{CO} 2$ & $f$ & $10 / 22 / 09$ & bw \\
\hline $\mathrm{n}$ & 3 & w6 & $\mathrm{CO} 2$ & $f$ & $9 / 20 / 09$ & bw \\
\hline $\mathrm{n}$ & 4 & w14 & pre-nutrient \& CO2 & $\mathrm{m}$ & $2 / 25 / 09$ & bw \\
\hline $\mathrm{n}$ & 4 & w14 & pre-nutrient \& CO2 & $\mathrm{m}$ & $4 / 9 / 09$ & bw \\
\hline $\mathrm{n}$ & 4 & w14 & pre-nutrient \& CO2 & $\mathrm{m}$ & $6 / 12 / 09$ & bw \\
\hline $\mathrm{n}$ & 4 & w14 & nutrient \&CO2 & $\mathrm{m}$ & $11 / 22 / 09$ & bw \\
\hline $\mathrm{n}$ & 4 & w26 & pre-ambient & $\mathrm{m}$ & $11 / 14 / 08$ & bw \\
\hline $\mathrm{n}$ & 4 & w26 & ambient & $\mathrm{m}$ & $7 / 4 / 09$ & bw \\
\hline $\mathrm{n}$ & 4 & w5 & $\mathrm{CO} 2$ & $\mathrm{~m}$ & $7 / 12 / 09$ & alk \\
\hline $\mathrm{n}$ & 4 & w5 & $\mathrm{co2}$ & $\mathrm{m}$ & $7 / 12 / 09$ & alk \\
\hline $\mathrm{n}$ & 4 & w5 & $\mathrm{CO} 2$ & $\mathrm{~m}$ & $9 / 20 / 09$ & bw \\
\hline$y$ & 5 & w13 & pre-ambient & $\mathrm{m}$ & $4 / 28 / 09$ & alk \\
\hline$y$ & 5 & w13 & pre-ambient & $\mathrm{m}$ & $6 / 12 / 09$ & bw \\
\hline $\mathrm{y}$ & 5 & w23 & ambient & $\mathrm{m}$ & $7 / 11 / 09$ & alk \\
\hline $\mathrm{y}$ & 5 & w77 & nutrient & $\mathrm{m}$ & 7/9/09 & alk \\
\hline $\mathrm{y}$ & 5 & w79 & pre-nutrient \& CO2 & $\mathrm{m}$ & $6 / 12 / 09$ & bw \\
\hline$y$ & 5 & w79 & nutrient \&CO2 & $\mathrm{m}$ & $7 / 10 / 09$ & alk \\
\hline $\mathrm{y}$ & 5 & w9 & pre-co2 & $\mathrm{m}$ & $4 / 22 / 09$ & alk \\
\hline $\mathrm{y}$ & 6 & w22 & pre-ambient & $\mathrm{m}$ & $11 / 14 / 08$ & bw \\
\hline $\mathrm{y}$ & 6 & w92 & pre-nutrient \& CO2 & $\mathrm{m}$ & $6 / 12 / 09$ & bw \\
\hline $\mathrm{y}$ & 6 & w92 & nutrient \&CO2 & $\mathrm{m}$ & $7 / 4 / 09$ & bw \\
\hline $\mathrm{y}$ & 7 & w49 & $\mathrm{CO} 2$ & $f$ & $10 / 22 / 09$ & bw \\
\hline $\mathrm{y}$ & 8 & w45 & pre-nutrient \& CO2 & $\mathrm{m}$ & $2 / 25 / 09$ & bw \\
\hline$y$ & 8 & w45 & pre-nutrient \& CO2 & $\mathrm{m}$ & $6 / 12 / 09$ & bw \\
\hline$y$ & 8 & w45 & nutrient \&CO2 & $\mathrm{m}$ & $7 / 4 / 09$ & bw \\
\hline $\mathrm{y}$ & 8 & w45 & nutrient \&CO2 & $\mathrm{m}$ & $7 / 10 / 09$ & alk \\
\hline $\mathrm{y}$ & 8 & w45 & nutrient \&CO2 & $\mathrm{m}$ & $8 / 13 / 09$ & bw \\
\hline
\end{tabular}


Table A-3 continued

\begin{tabular}{|c|c|c|c|c|c|c|}
\hline zooxanthellae & parent & fragment & treatment & gender & date & observ. \\
\hline$y$ & 8 & w57 & pre-ambient & $\mathrm{m}$ & $11 / 14 / 08$ & bw \\
\hline$y$ & 8 & w57 & pre-ambient & $\mathrm{m}$ & $4 / 28 / 09$ & alk \\
\hline$y$ & 8 & w57 & pre-ambient & $\mathrm{m}$ & $6 / 12 / 09$ & bw \\
\hline$y$ & 8 & w57 & ambient & $\mathrm{m}$ & $7 / 11 / 09$ & alk \\
\hline$y$ & 8 & w57 & ambient & $\mathrm{m}$ & $8 / 13 / 09$ & bw \\
\hline$y$ & 8 & w58 & pre-co2 & $\mathrm{m}$ & $4 / 9 / 09$ & bw \\
\hline$y$ & 8 & w58 & $\mathrm{CO} 2$ & $\mathrm{~m}$ & $9 / 20 / 09$ & bw \\
\hline$y$ & 8 & w58 & $\mathrm{CO} 2$ & $\mathrm{~m}$ & $10 / 22 / 09$ & bw \\
\hline
\end{tabular}



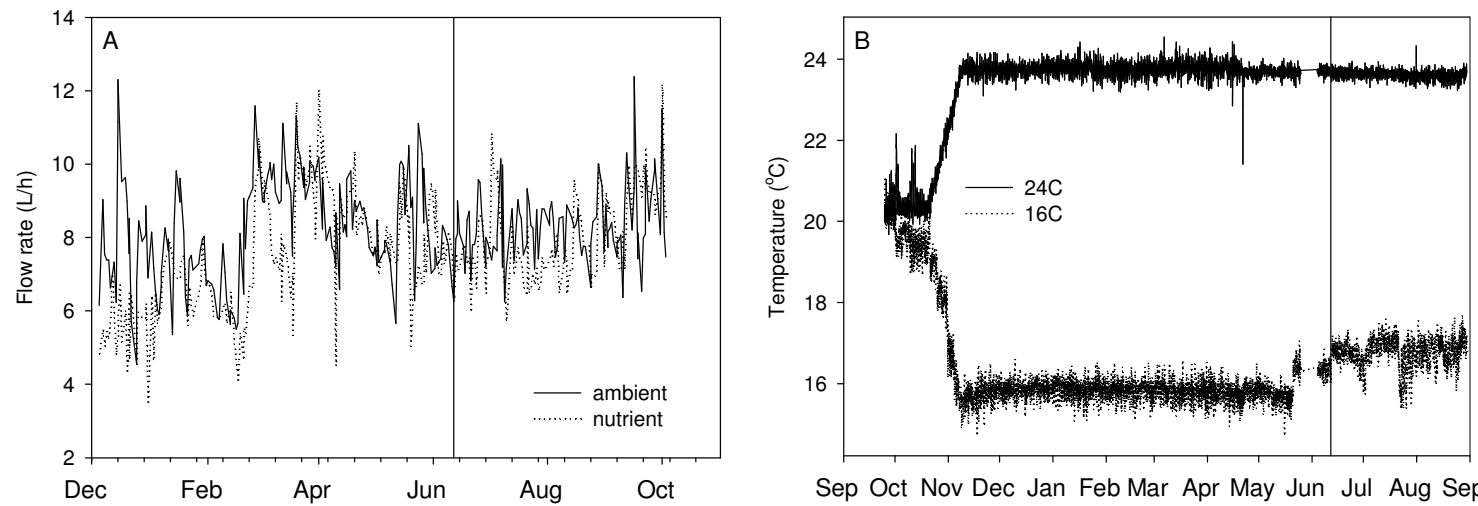

Sep Oct Nov Dec Jan Feb Mar Apr May Jun Jul Aug Sep
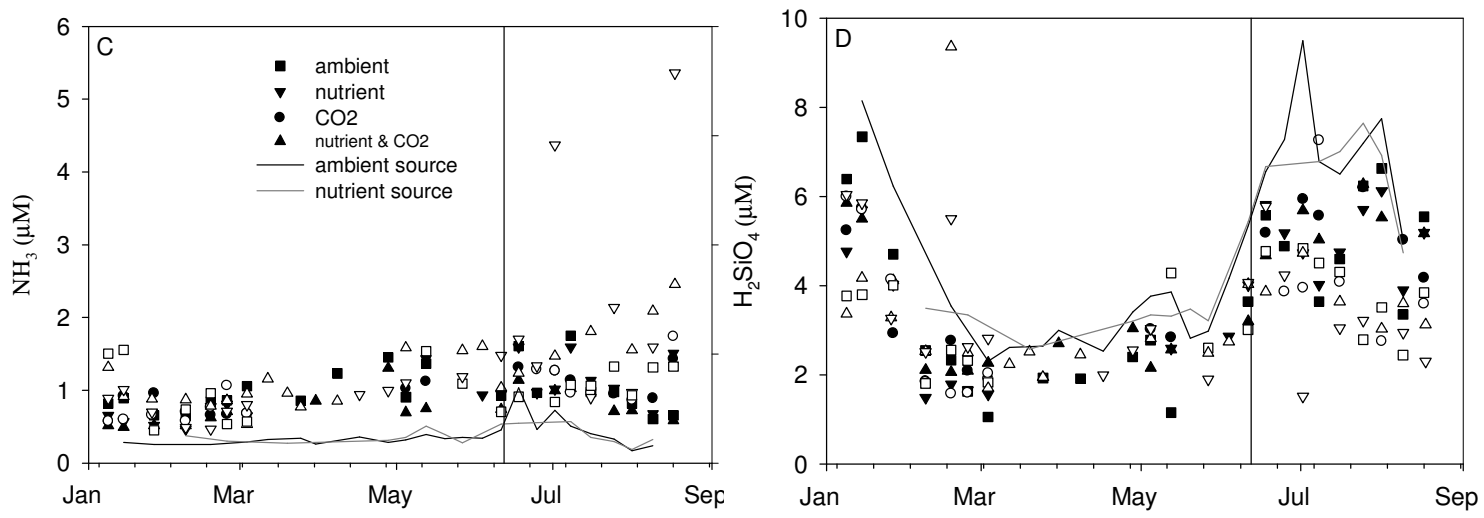

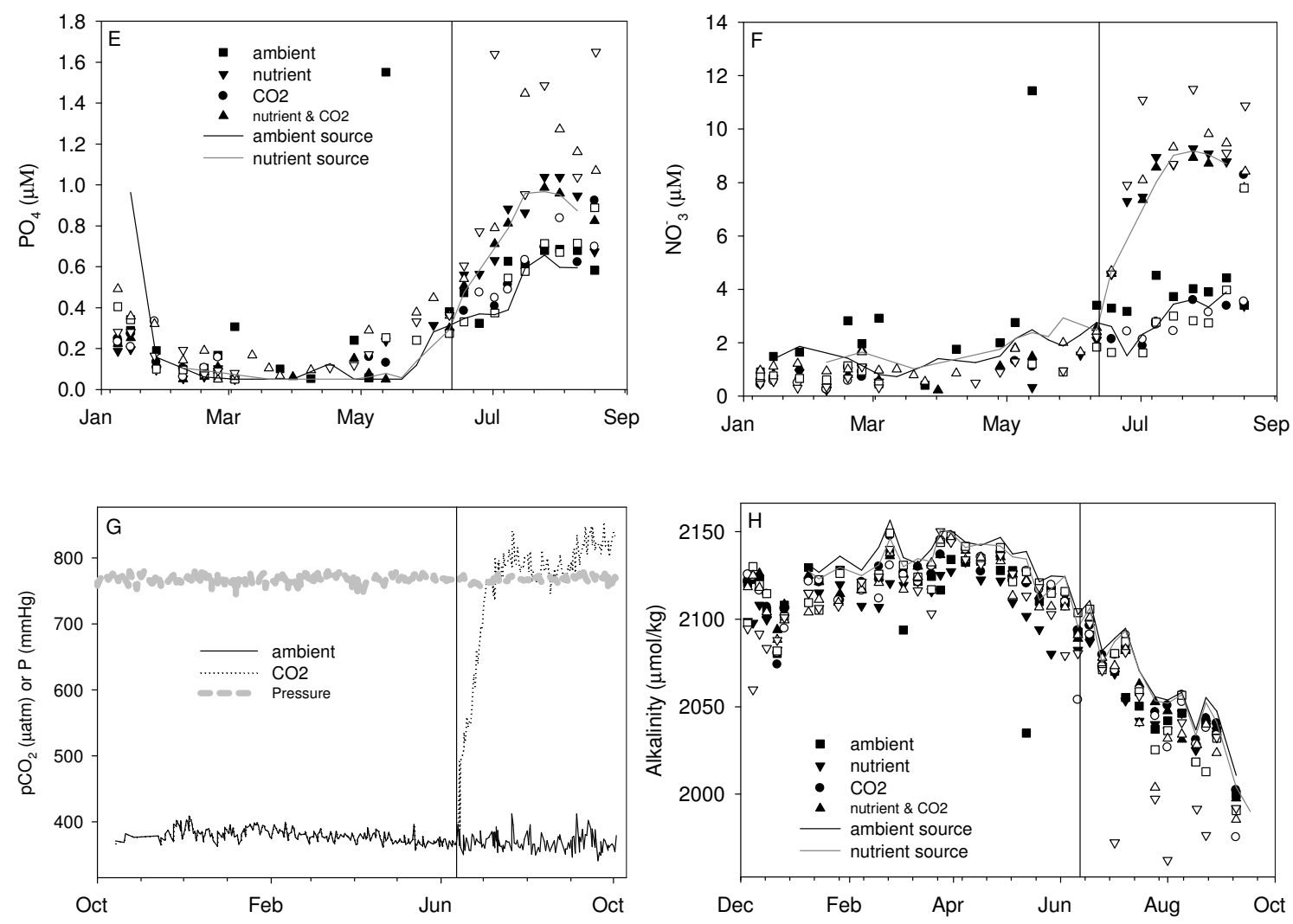

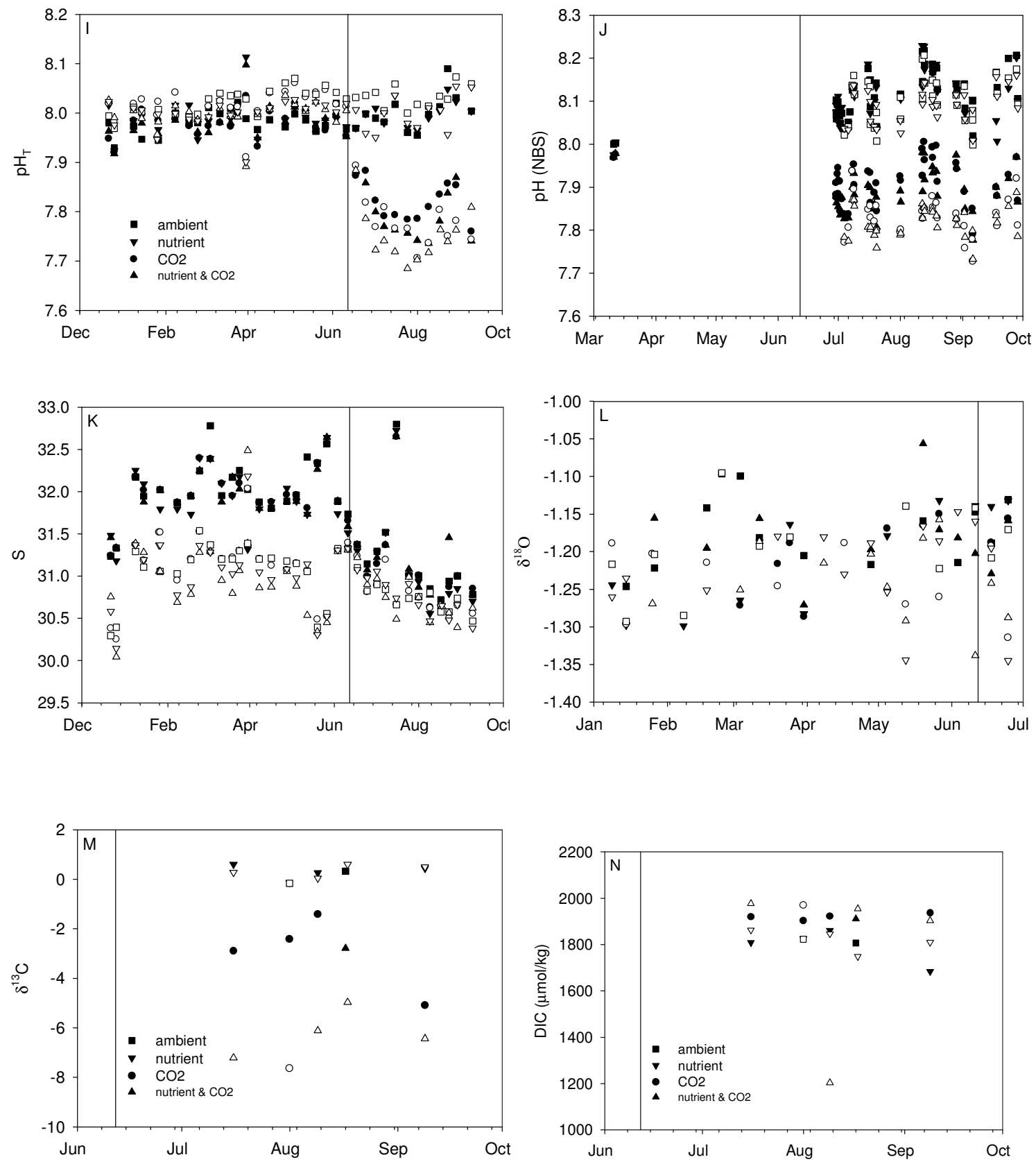
Figure A-1. Aquarium conditions over time. A. Water in each of the two reservoirs supplying the aquarium system was replenished on a daily basis and the volume used between fillings measured, this is plotted as an hourly flow rate versus time starting in Dec. 2008. B. A temperature logger recorded temperature every $12 \mathrm{~min}$ throughout the experiment starting before corals were added in September 2008. Plotted values are hourly averages. Data were averaged to reduce artificial variability caused by electrical interference generated by fluorescent lighting. C. Ammonia concentrations measured both within each aquarium and in the reservoirs supplying the aquaria. Open symbols are used for $16^{\circ} \mathrm{C}$ treatments, closed for $24^{\circ} \mathrm{C}$ treatments. D. Silicate concentrations measured within each aquarium and in the reservoirs, symbols are as in Fig. A- 1C. E. Phosphate concentrations measured within each aquarium and in the reservoirs, symbols are as in Fig. A- 1C. F. Nitrate/nitrite concentrations measured within each aquarium and in the reservoirs, symbols are as in Fig. A- 1C. G. Gas $\mathrm{CO}_{2}$ partial pressures ( $\left.\mu \mathrm{atm}\right)$ and total atmospheric pressure $(\mathrm{mmHg})$ measured on a daily basis on the supply lines for both the ambient and elevated $\mathrm{pCO}_{2}$ treatments. H. Alkalinity concentrations measured within each aquarium and in the reservoirs, symbols are as in Fig. A- 1C. I. pH values measured spectrophotometrically within each aquarium, symbols are as in Fig. A- 1C. J. $\mathrm{pH}$ values measured with an electrode within each aquarium on light/dark cycles, symbols are as in Fig. A- 1C. K. Salinity values measured within each aquarium. L. $\mathrm{d}^{18} \mathrm{O}$ values measured within each aquarium, symbols are as in Fig. A- $1 \mathrm{C} . \mathrm{M} \cdot \delta^{13} \mathrm{C}$ DIC values measured within each aquarium. N. DIC concentrations measured within each aquarium. Data points are individual measurements, except for alkalinity data for which 
averages of replicate measurements are plotted, standard deviation for each point is less than $5 \mu \mathrm{mol} / \mathrm{kg}$. The vertical line indicates the start of treatments. 

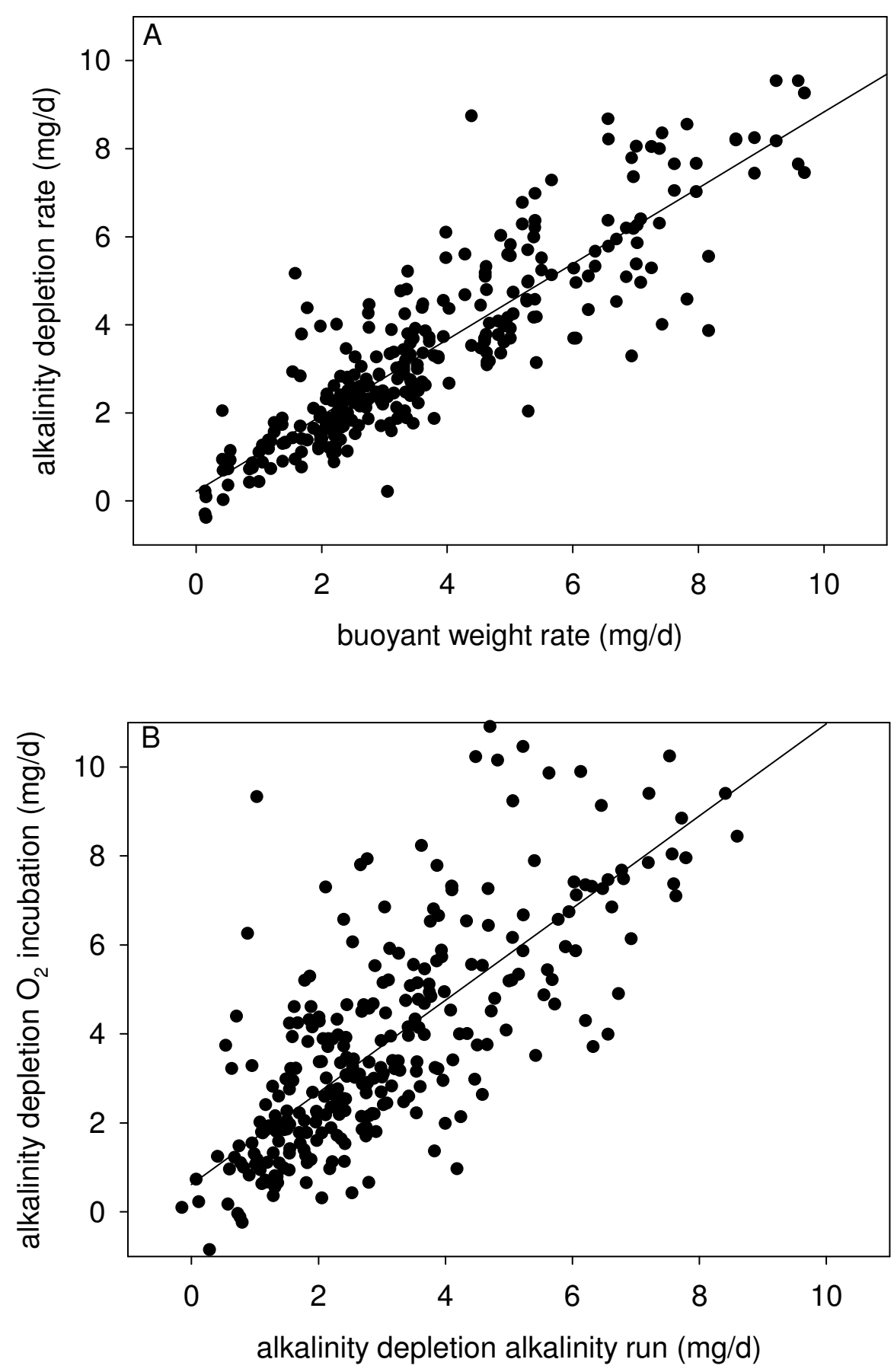
Figure A-2. Comparisons of calcification rate measurement techniques. A. Relationship between buoyant weight measurements and daily averaged alkalinity depletion rate measurements. B. Relationship between alkalinity depletion rate measurements from alkalinity runs and alkalinity depletion rate measurements from oxygen incubations. 

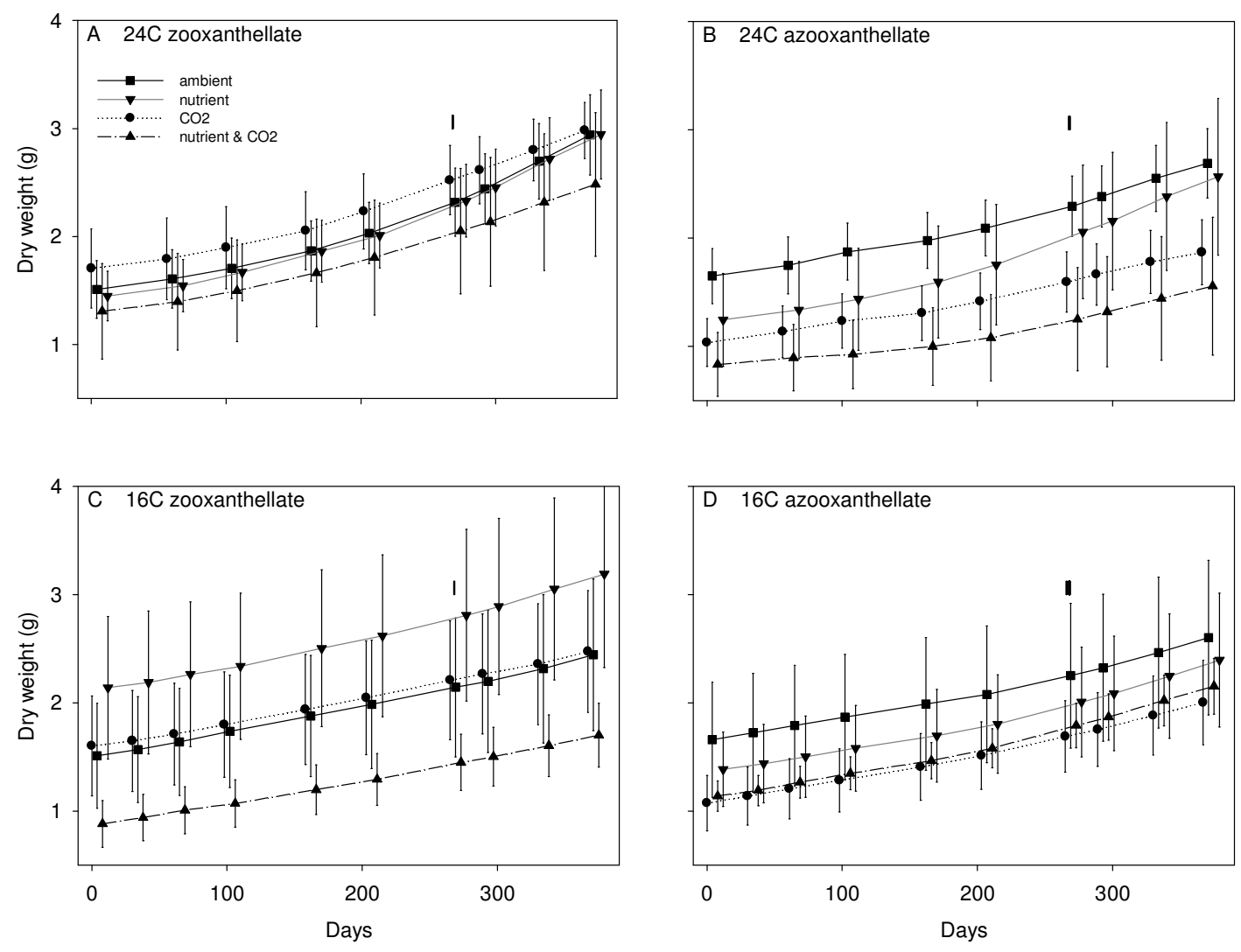

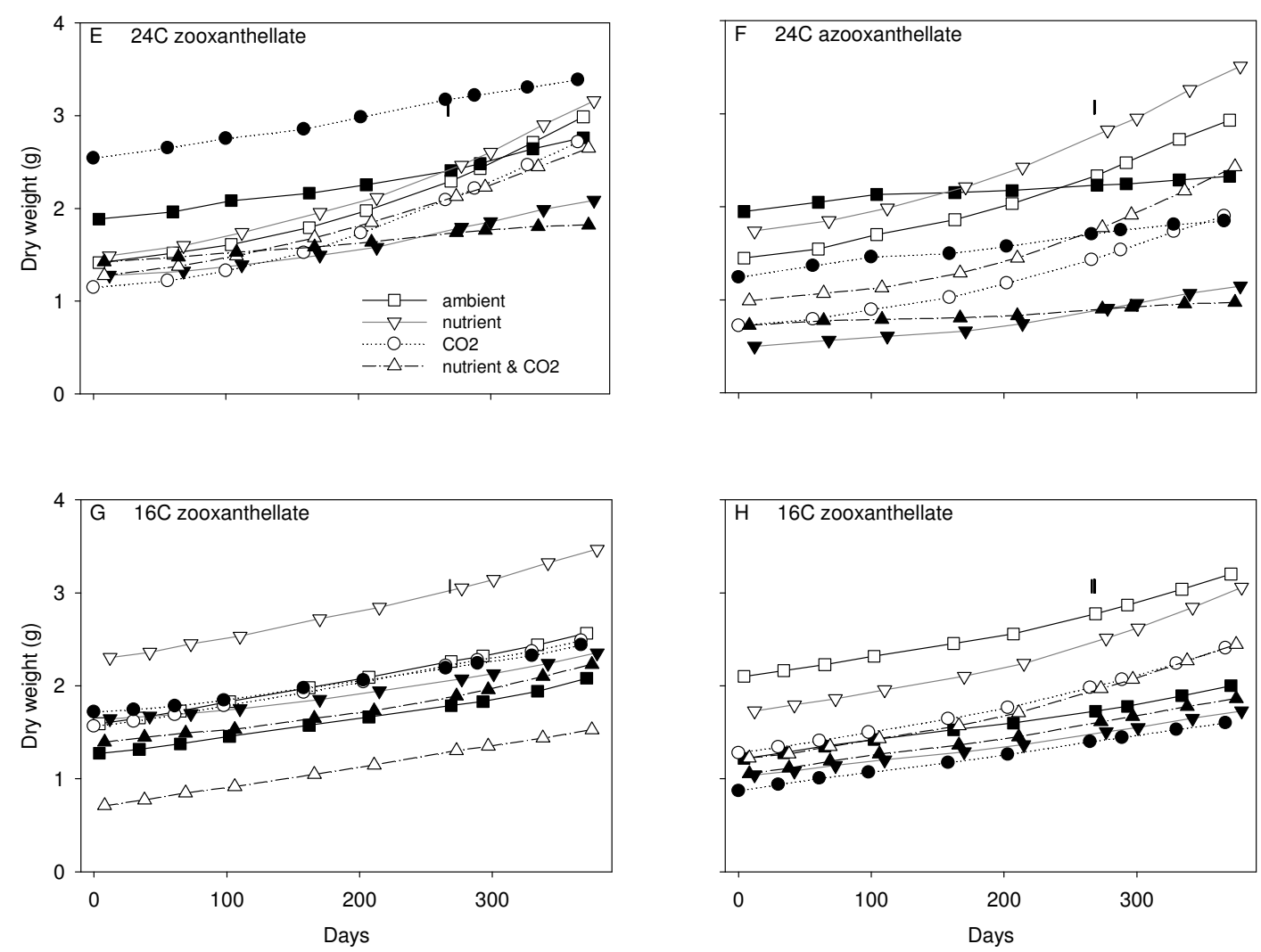

Figure A-3. Calculated coral dry weights measured over the course of the experiment, AD show average weights for all corals, E-H show averages by gender. A. Dry weights for zooxanthellate corals maintained at $24^{\circ} \mathrm{C}$. B. Dry weights for azooxanthellate corals maintained at $24^{\circ} \mathrm{C}$. C. Dry weights for zooxanthellate corals maintained at $16^{\circ} \mathrm{C}$. D. Dry weights for azooxanthellate corals maintained at $16^{\circ} \mathrm{C}$. E. Dry weights for zooxanthellate corals maintained at $24^{\circ} \mathrm{C}$ separated by gender. Average weights for males are shown with open symbols, females are shown with filled symbols. F. Dry weights for azooxanthellate corals maintained at $24^{\circ} \mathrm{C}$ separated by gender, symbols as used in 3E. G. Dry weights for zooxanthellate corals maintained at $16^{\circ} \mathrm{C}$ separated by gender, symbols as used in 3E. H. Dry weights for azooxanthellate corals maintained at 
$16^{\circ} \mathrm{C}$ separated by gender, symbols as used in 3E. A vertical bar on each plot move to $\mathbf{y}$ axis marks the start of treatments. Error bars are standard error and are omitted on plots E-H for clarity. Weights are offset on the time axis to separate treatment symbols, at each time measurements were made, all corals in all treatments were weighed. Day 0 marks the day corals were collected and initial weight measurements made. 

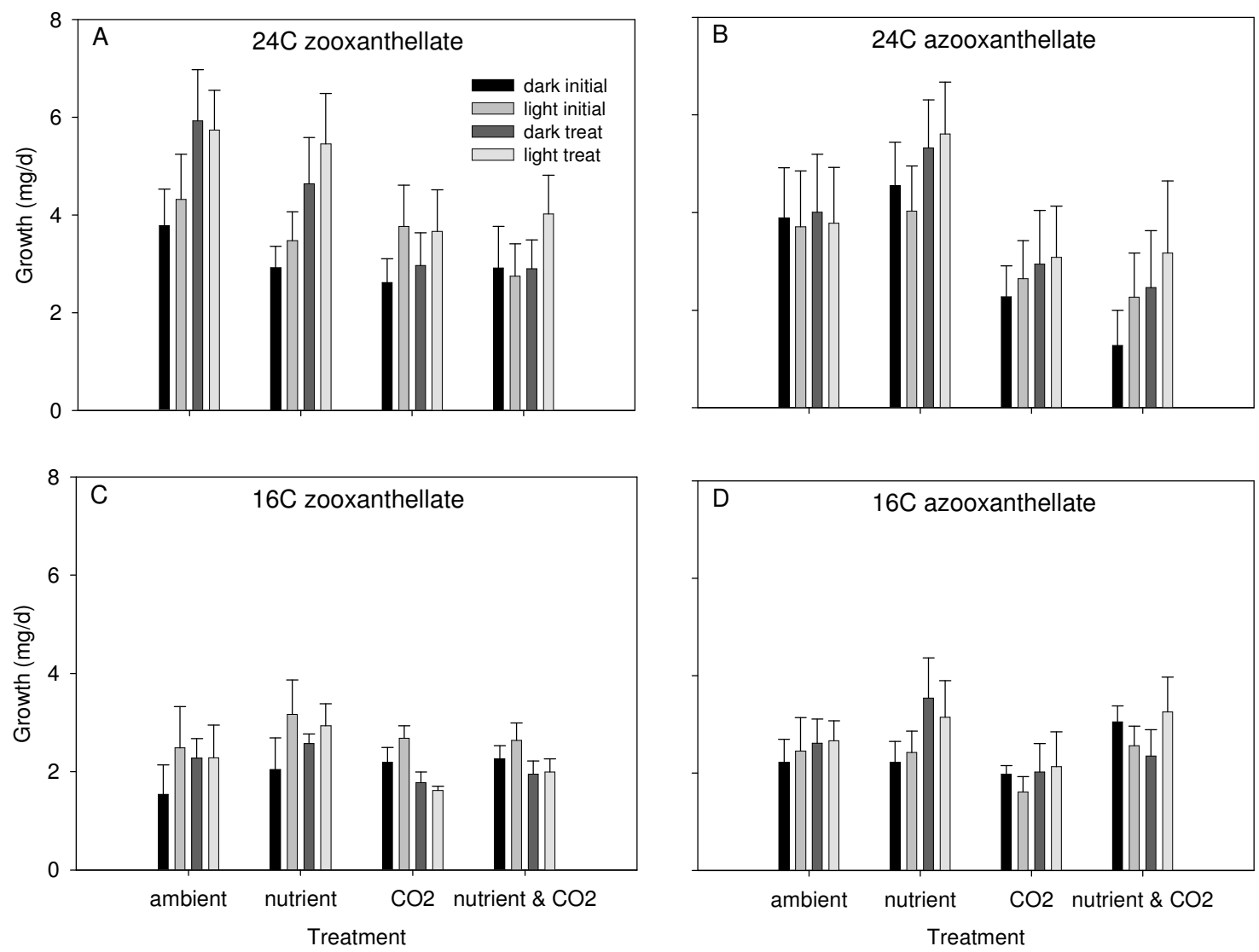

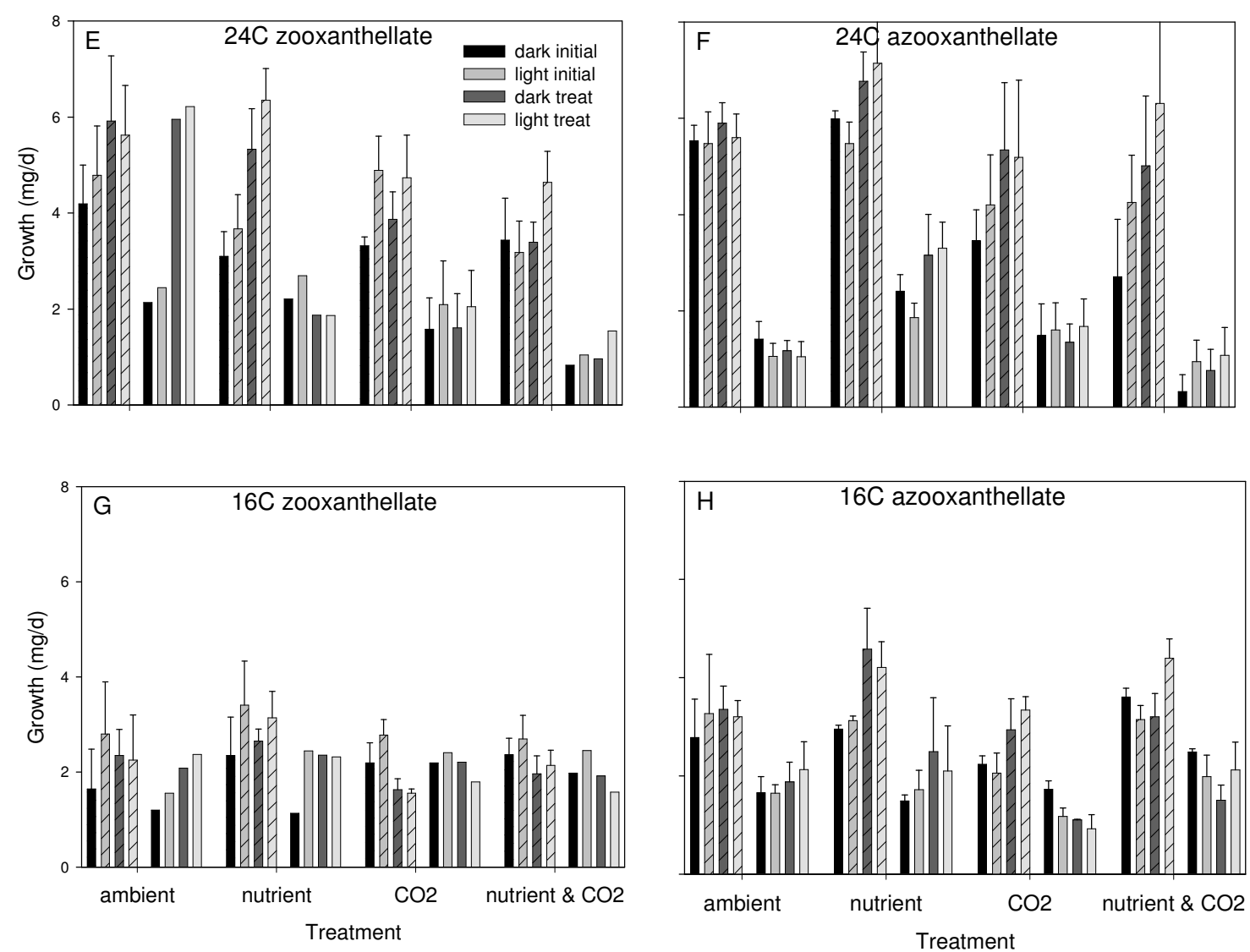

Figure A-4. Coral calcification rates for each treatment estimated from alkalinity uptake measurements before (initial) and after the start of treatments (treat) in the light and dark for alkalinity depletion runs. A-D show average rates for all corals, E-H show averages by gender. A. Growth rates for zooxanthellate corals maintained at $24^{\circ} \mathrm{C}$. B. Growth rates for azooxanthellate corals maintained at $24^{\circ} \mathrm{C}$. C. Growth rates for zooxanthellate corals maintained at $16^{\circ} \mathrm{C}$. D. Growth rates for azooxanthellate corals maintained at $16^{\circ} \mathrm{C}$. E. Growth rates for zooxanthellate corals maintained at $24^{\circ} \mathrm{C}$ separated by gender. Average growth rates for males are shown with bars with hatch marks (left set of bars for each treatment), females are shown with solid bars (right set of bars for each treatment). 
F. Growth rates for azooxanthellate corals maintained at $24^{\circ} \mathrm{C}$ separated by gender, symbols as used in $3 \mathrm{E}$. G. Growth rates for zooxanthellate corals maintained at $16^{\circ} \mathrm{C}$ separated by gender, symbols as used in 3E. H. Growth rates for azooxanthellate corals maintained at $16^{\circ} \mathrm{C}$ separated by gender, symbols as used in 3E. Within each treatment group, the left-most bar (black) is the initial dark rate, the next bar is the initial rate in the light (grey), then next bar (dark grey) is treatment rate in the dark, and the right-most bar (light grey) is the treatment rate in the light. Bars are means and error bars are standard error. 

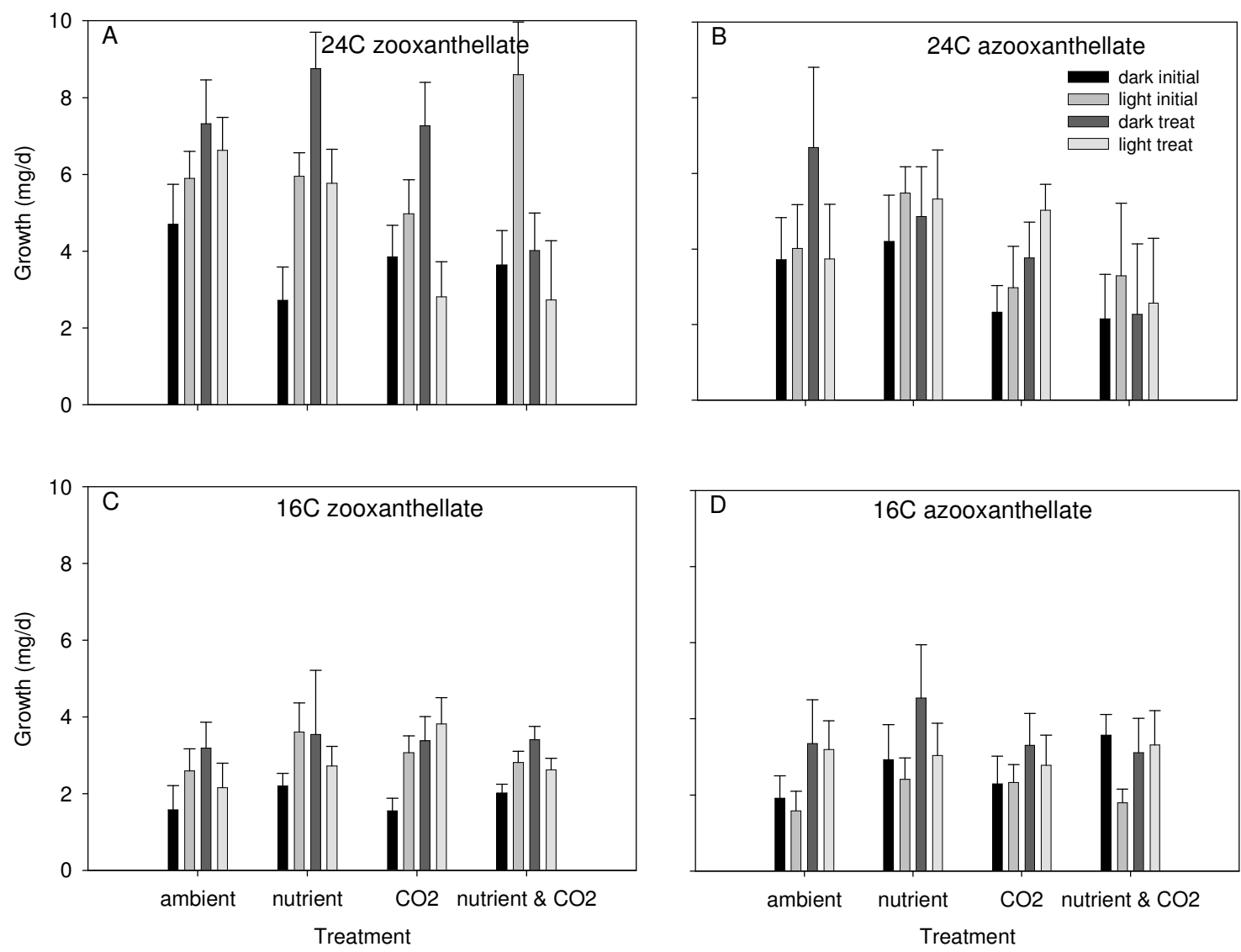

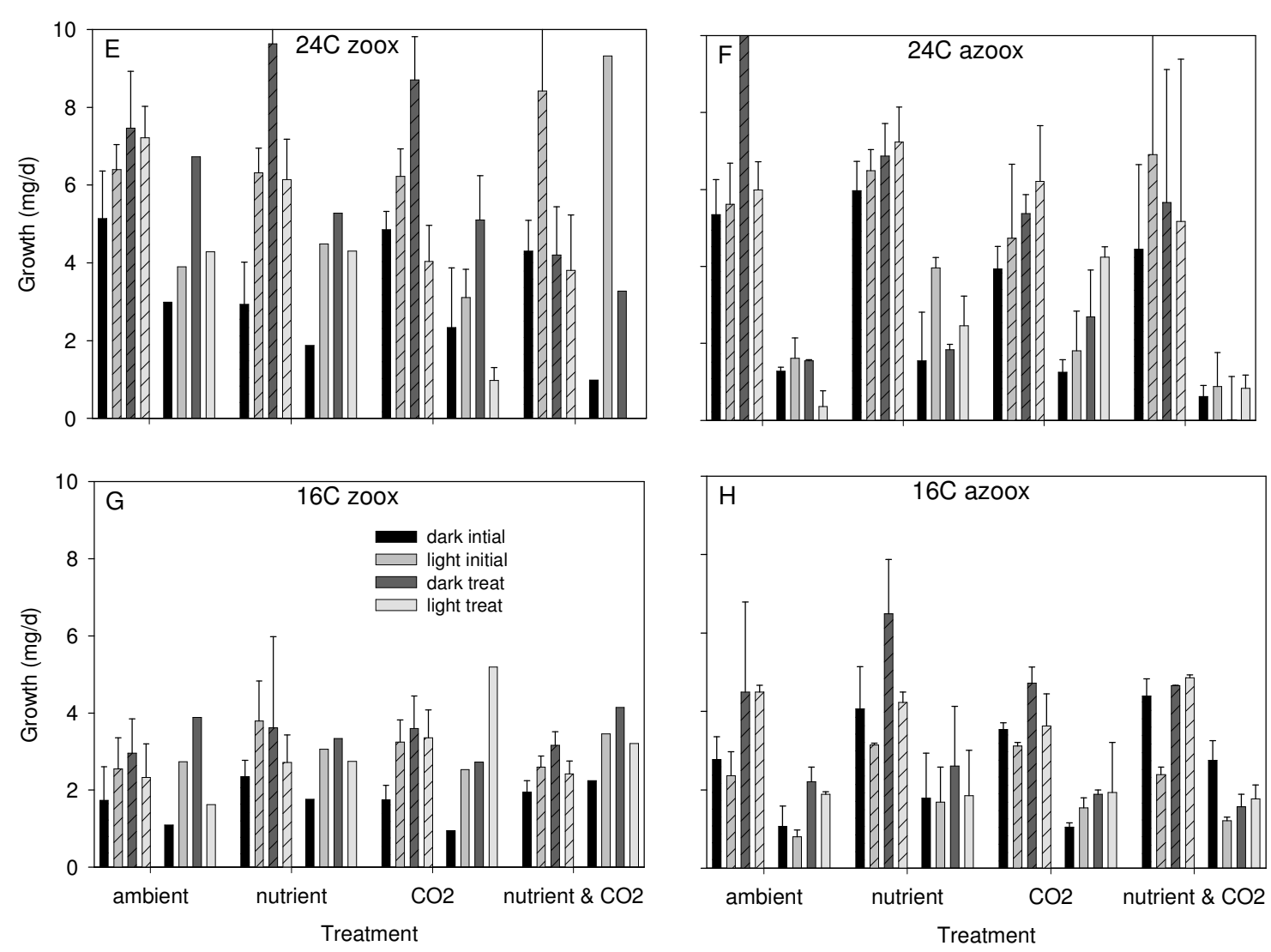

Figure A-5. Coral calcification rates for each treatment estimated from alkalinity uptake measurements before (initial) and after the start of treatments (treat) in the light and dark for oxygen incubations. A-D show average rates for all corals, E-H show averages by gender. A. Growth rates for zooxanthellate corals maintained at $24^{\circ} \mathrm{C}$. B. Growth rates for azooxanthellate corals maintained at $24^{\circ} \mathrm{C}$. C. Growth rates for zooxanthellate corals maintained at $16^{\circ} \mathrm{C}$. D. Growth rates for azooxanthellate corals maintained at $16^{\circ} \mathrm{C}$. E. Growth rates for zooxanthellate corals maintained at $24^{\circ} \mathrm{C}$ separated by gender. Average growth rates for males are shown with bars with hatch marks (left set of bars for each treatment), females are shown with solid bars (right set of bars for each treatment). F. Growth rates for azooxanthellate corals maintained at $24^{\circ} \mathrm{C}$ separated by gender, symbols 
as used in $3 \mathrm{E}$. G. Growth rates for zooxanthellate corals maintained at $16^{\circ} \mathrm{C}$ separated by gender, symbols as used in 3E. H. Growth rates for azooxanthellate corals maintained at $16^{\circ} \mathrm{C}$ separated by gender, symbols as used in 3E. Within each treatment group, the leftmost bar (black) is the initial dark rate, the next bar is the initial rate in the light (grey), then next bar (dark grey) is treatment rate in the dark, and the right-most bar (light grey) is the treatment rate in the light. Bars are means and error bars are standard error. 

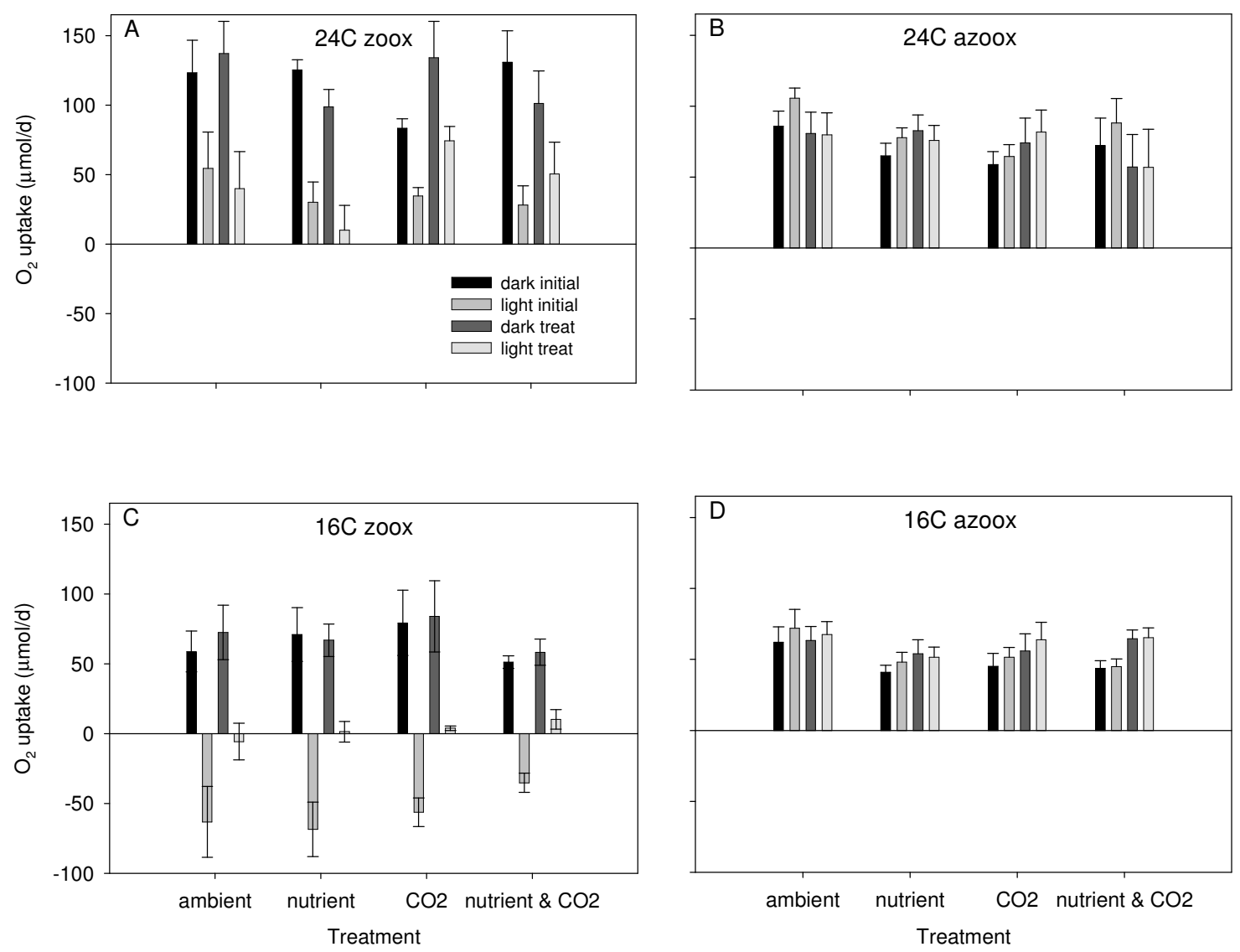

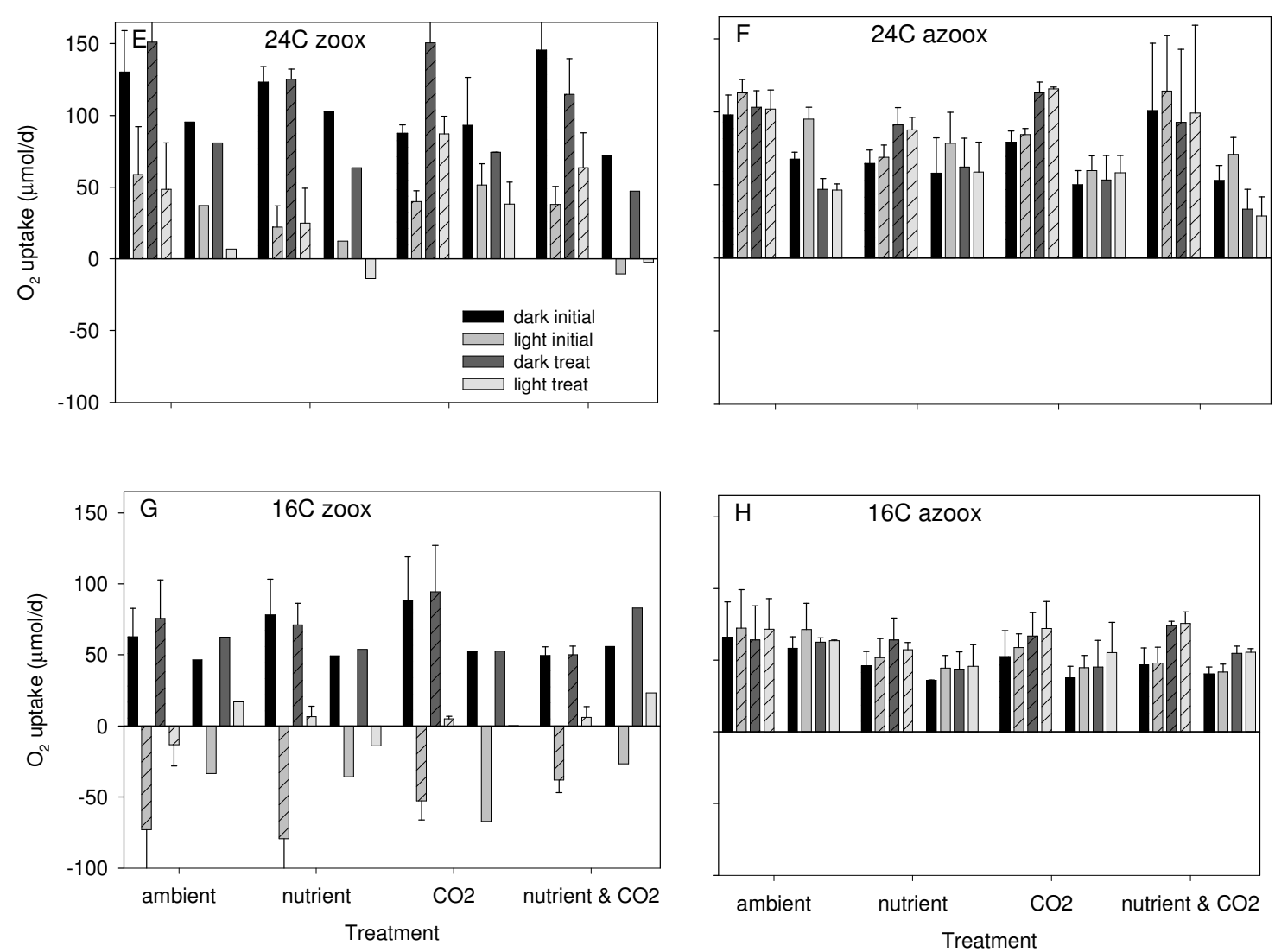

Figure A-6. Coral oxygen consumption rates for each treatment before (initial) and after the start of treatments (treat) in the light and dark. A-D show average rates for all corals, E-H show averages by gender. A. Oxygen consumption rates for zooxanthellate corals maintained at $24^{\circ} \mathrm{C}$. B. Oxygen consumption rates for azooxanthellate corals maintained at $24^{\circ} \mathrm{C}$. C. Oxygen consumption rates for zooxanthellate corals maintained at $16^{\circ} \mathrm{C}$. D. Oxygen consumption rates for azooxanthellate corals maintained at $16^{\circ} \mathrm{C}$. E. Growth rates for zooxanthellate corals maintained at $24^{\circ} \mathrm{C}$ separated by gender. Average Oxygen consumption rates for males are shown with bars with hatch marks (left set of bars for each treatment), females are shown with solid bars (right set of bars for each treatment). F. Oxygen consumption rates for azooxanthellate corals maintained at $24^{\circ} \mathrm{C}$ separated by 
gender, symbols as used in 3E. G. Oxygen consumption rates for zooxanthellate corals maintained at $16^{\circ} \mathrm{C}$ separated by gender, symbols as used in 3E. H. Oxygen consumption rates for azooxanthellate corals maintained at $16^{\circ} \mathrm{C}$ separated by gender, symbols as used in 3E. Within each treatment group, the left-most bar (black) is the initial dark rate, the next bar is the initial rate in the light (grey), then next bar (dark grey) is treatment rate in the dark, and the right-most bar (light grey) is the treatment rate in the light. Bars are means and error bars are standard error. 

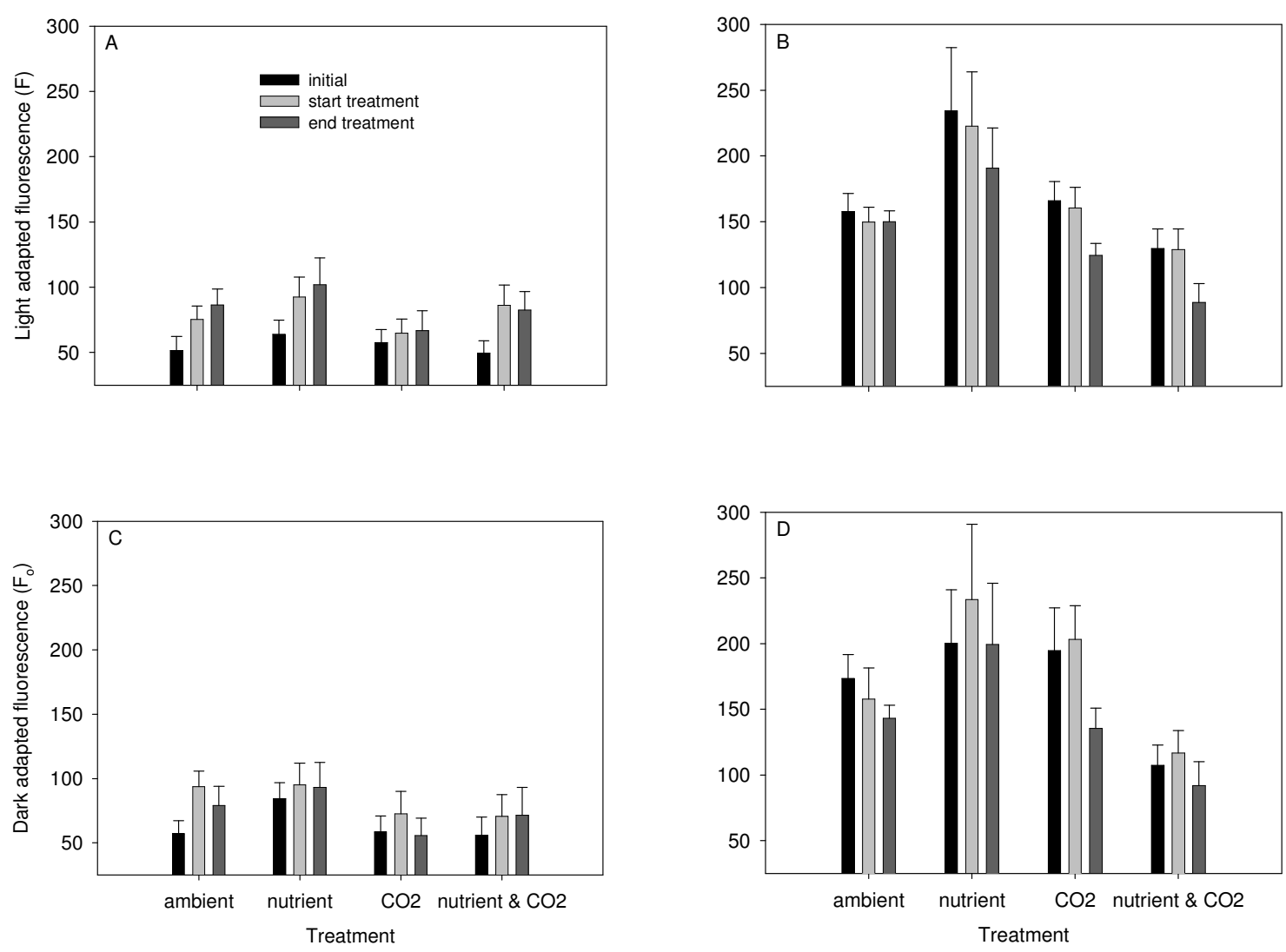

Figure A-7. Average light (A,B) and dark (C,D) adapted coral fluorescence levels measured in each treatment before the start of treatments (initial), at the start of treatment conditions (start treatment), and two months later at the end of the experiment (end treatment). A. Light adapted fluorescence levels for zooxanthellate corals maintained at $24^{\circ} \mathrm{C}$. B. Light adapted fluorescence levels for zooxanthellate corals maintained at $16^{\circ} \mathrm{C}$. C. Dark adapted fluorescence levels for zooxanthellate corals maintained at $24^{\circ} \mathrm{C}$. D. Dark adapted fluorescence levels for zooxanthellate corals maintained at $16^{\circ} \mathrm{C}$. Bars are average values, error bars are standard error. 

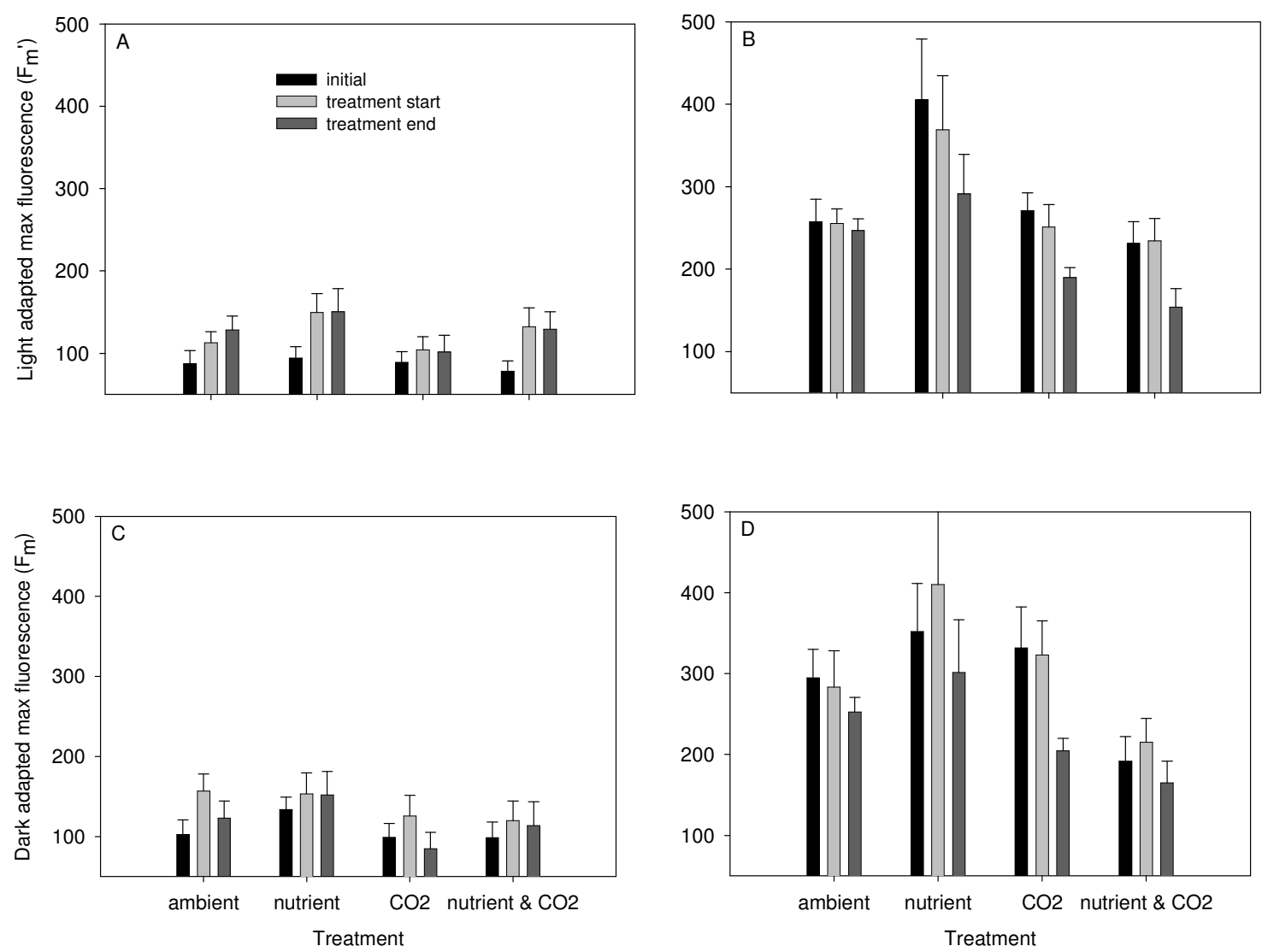

Figure A-8. Average light (A,B) and dark (C,D) adapted coral maximal fluorescence levels measured in each treatment before the start of treatments (initial), at the start of treatment conditions (start treatment), and two months later at the end of the experiment (end treatment). A. Light adapted fluorescence levels for zooxanthellate corals maintained at $24^{\circ} \mathrm{C}$. B. Light adapted fluorescence levels for zooxanthellate corals maintained at $16^{\circ} \mathrm{C}$. C. Dark adapted fluorescence levels for zooxanthellate corals maintained at $24^{\circ} \mathrm{C}$. D. Dark adapted fluorescence levels for zooxanthellate corals maintained at $16^{\circ} \mathrm{C}$. Bars are average values, error bars are standard error. 

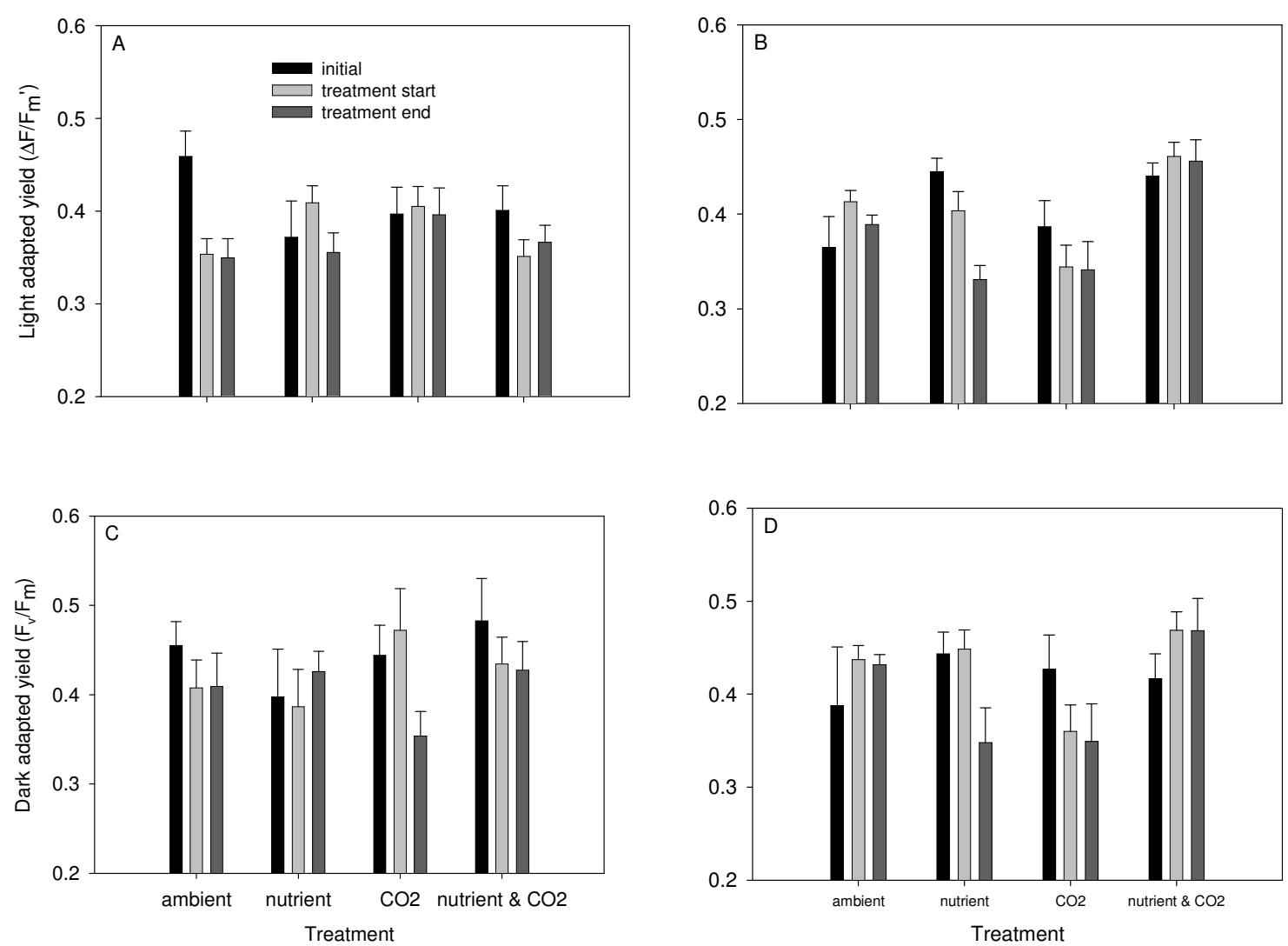

Figure A-9. Average light (A,B) and dark (C,D) adapted quantum yields measured in each treatment before the start of treatments (initial), at the start of treatment conditions (start treatment), and two months later at the end of the experiment (end treatment). A. Light adapted quantum yields for zooxanthellate corals maintained at $24^{\circ} \mathrm{C}$. B. Light adapted quantum yields for zooxanthellate corals maintained at $16^{\circ} \mathrm{C}$. C. Dark adapted quantum yields for zooxanthellate corals maintained at $24^{\circ} \mathrm{C}$. D. Dark adapted quantum yields for zooxanthellate corals maintained at $16^{\circ} \mathrm{C}$. Bars are average values, error bars are standard error. 

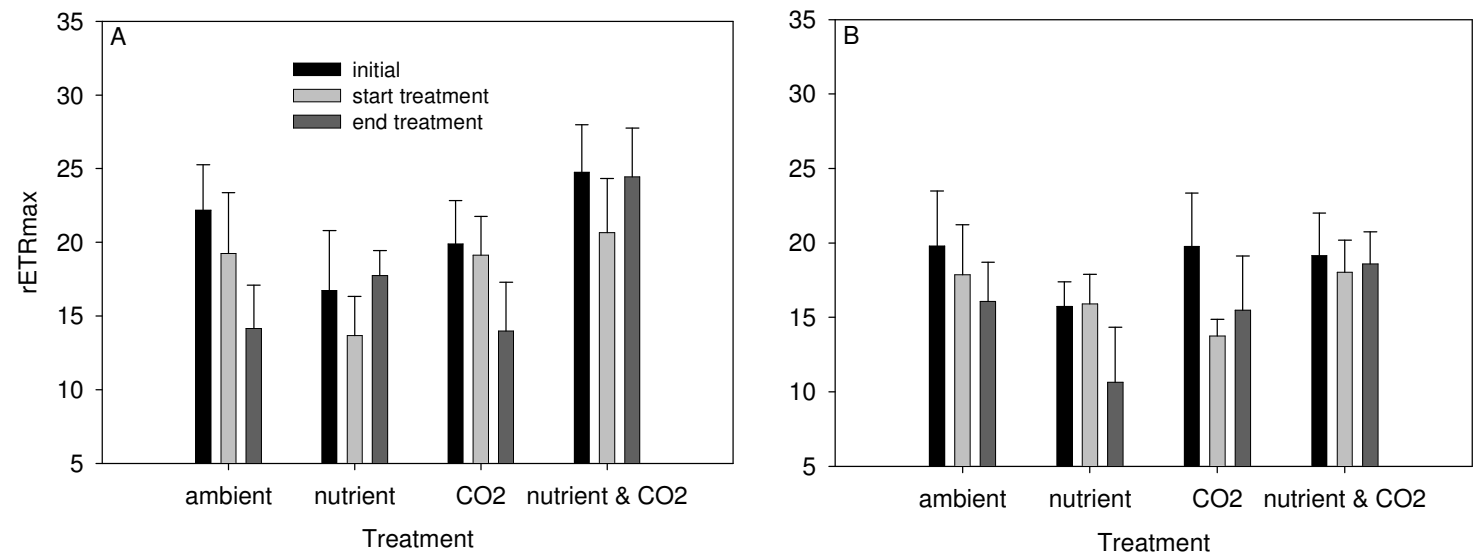

Figure A-10. Average maximum relative electron transport rates (rETRmax) for zooxanthellate corals maintained at $24^{\circ} \mathrm{C}(\mathrm{A})$ and $16^{\circ} \mathrm{C}(\mathrm{B})$ measured in each treatment before the start of treatments (initial), at the start of treatment conditions (start treatment), and two months later at the end of the experiment (end treatment). Bars are average values, error bars are standard error. 

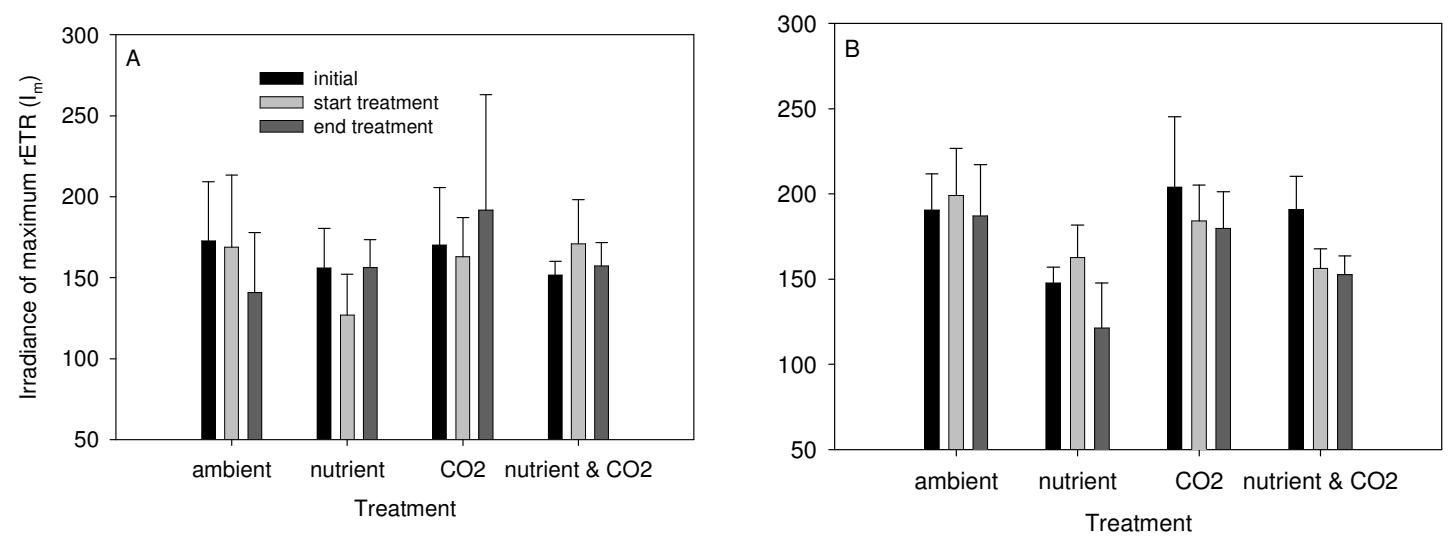

Figure A-11. Average irradiance of maximal relative electron transport rate (Im) for zooxanthellate corals maintained at $24^{\circ} \mathrm{C}(\mathrm{A})$ and $16^{\circ} \mathrm{C}(\mathrm{B})$ measured in each treatment before the start of treatments (initial), at the start of treatment conditions (start treatment), and two months later at the end of the experiment (end treatment). Bars are average values, error bars are standard error. 

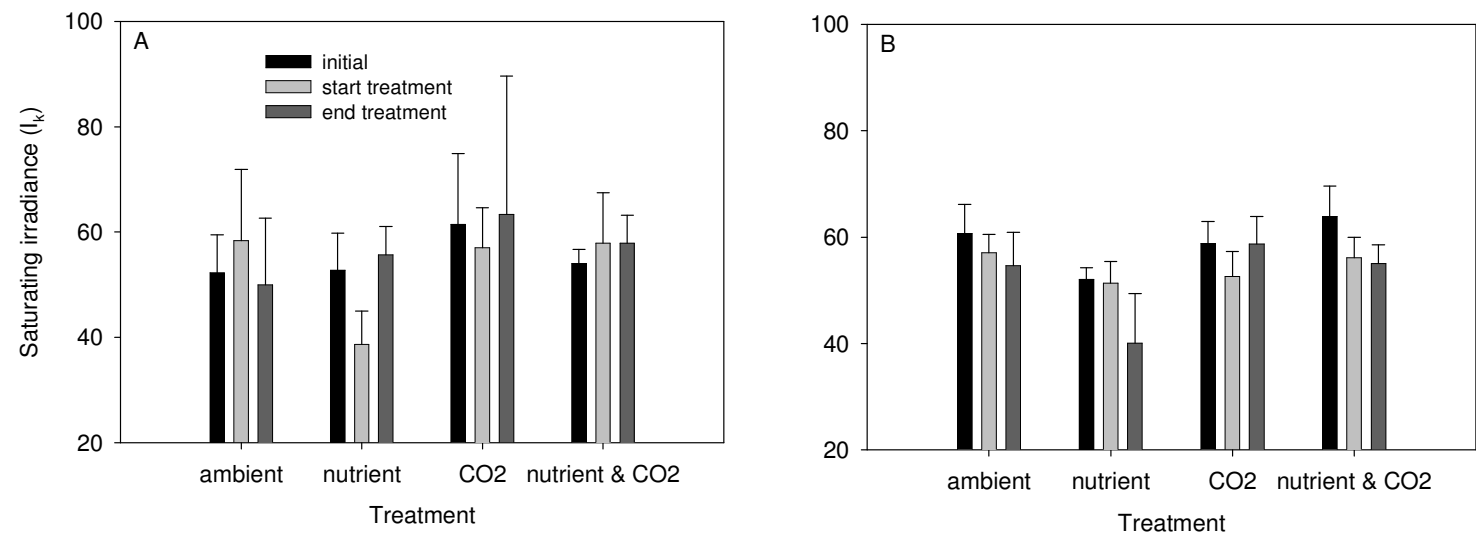

Figure A-12. Average saturating irradiance (Ik) for zooxanthellate corals maintained at $24^{\circ} \mathrm{C}(\mathrm{A})$ and $16^{\circ} \mathrm{C}(\mathrm{B})$ measured in each treatment before the start of treatments (initial), at the start of treatment conditions (start treatment), and two months later at the end of the experiment (end treatment). Bars are average values, error bars are standard error. 

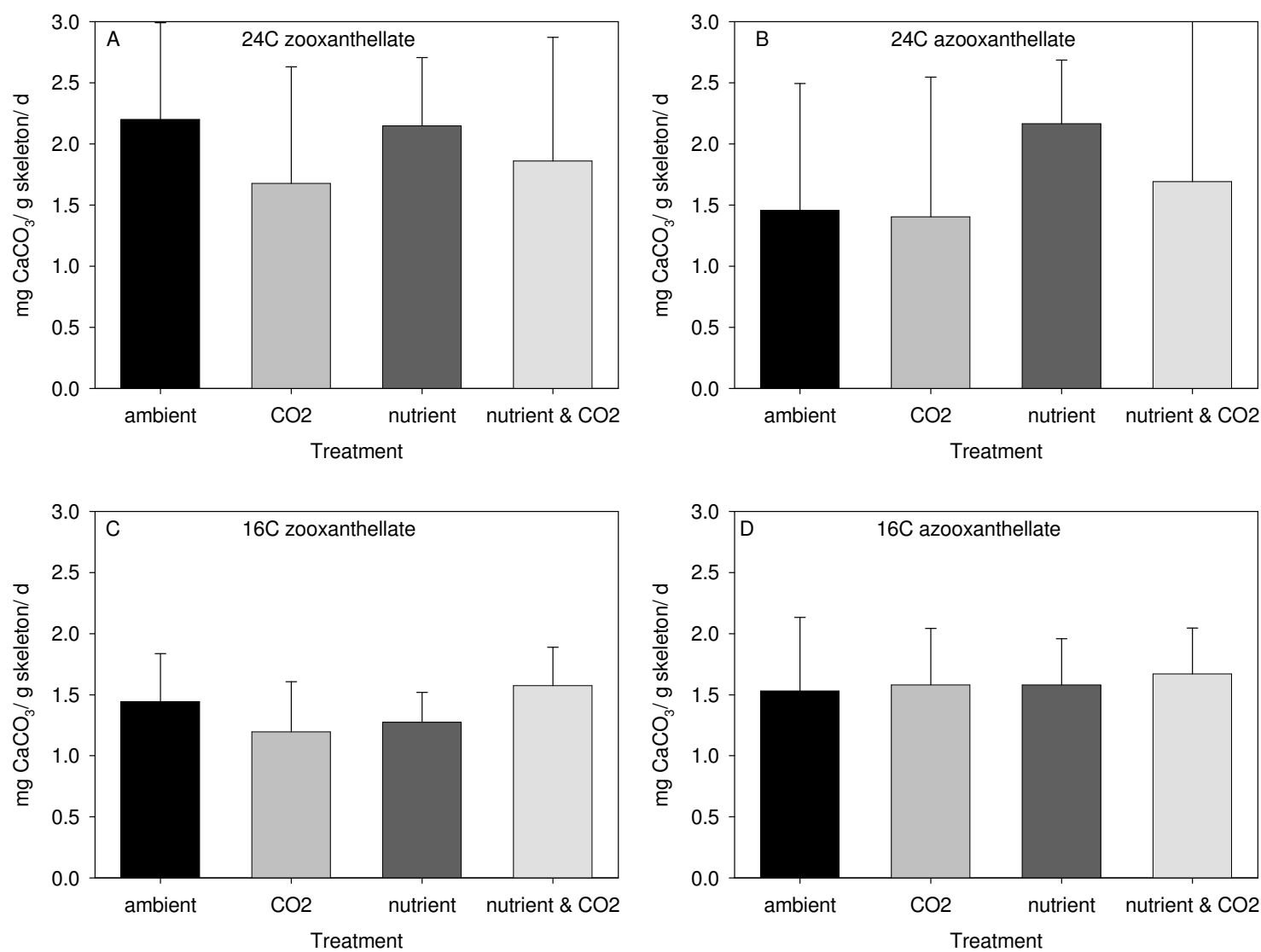

Figure A-13. Mass normalized calcification rates for each treatment. Data are expressed as $\mathrm{mg} \mathrm{CaCO}_{3}$ deposited per day during the treatment phase per $\mathrm{g}$ of final skeletal dry weight. A. Growth rates for zooxanthellate corals maintained at $24^{\circ} \mathrm{C}$. B. Growth rates for azooxanthellate corals maintained at $24^{\circ} \mathrm{C}$. C. Growth rates for zooxanthellate corals maintained at $16^{\circ} \mathrm{C}$. D. Growth rates for azooxanthellate corals maintained at $16^{\circ} \mathrm{C}$. Bars represent means, error bars are standard deviation. 


\title{
Appendix 5: paper based on Chapter 4
}

Gender bias in the coral response to ocean acidification

\begin{abstract}
Ocean acidification reduces coral calcification and threatens coral communities worldwide. Here, spawning female colonies of the coral Astrangia poculata are shown to be more sensitive to ocean acidification than males. The heightened sensitivity of female coral calcification appears linked to spawning. Whereas ocean acidification had similar effects on calcification rates of non-spawning males and females, a pronounced gender difference was observed in spawning corals, with female calcification showing a significant reduction under elevated $\mathrm{pCO}_{2}$. The observed gender difference in spawning corals may be linked to the energy requirements of egg production, which could limit the energy available for calcification.
\end{abstract}

Submitted to Nature as: Holcomb, M., Cohen, A.L., McCorkle, D.C., Gender bias in the coral response to ocean acidification. 
Anthropogenic production of $\mathrm{CO}_{2}$ is associated with rising levels of $\mathrm{CO}_{2}$ in the atmosphere and in-turn increased $\mathrm{CO}_{2}$ concentrations in the oceans ${ }^{1}$. As $\mathrm{CO}_{2}$ concentrations rise, $\mathrm{pH}$ declines, this decline in $\mathrm{pH}$ (called ocean acidification) represents one of the single greatest concerns for the survival of marine organisms world-wide ${ }^{2}$. Coral communities in particular have been a source of much concern as ocean acidification reduces the aragonite saturation state and in-turn reduces coral growth (calcification) rates ${ }^{3,4}$. Despite considerable research on the effects of ocean acidification on numerous organisms, no study has thus far addressed whether the effects differ between genders. Here, spawning female colonies of the coral Astrangia poculata are shown to be more sensitive to ocean acidification than males. The heightened sensitivity of female coral calcification appears linked to spawning. Whereas ocean acidification had similar effects on calcification rates of non-spawning males and females, a pronounced gender difference was observed in spawning corals, with female calcification showing a significant reduction under elevated $\mathrm{pCO}_{2}$. The observed gender difference in spawning corals may be linked to the energy requirements of egg production, which could limit the energy available for calcification. These findings suggest gender based differences may be a significant and as yet unexplored factor determining how the population structure of marine organisms will change in response to ocean acidification. Ocean acidification may alter gender ratios, potentially making organisms more vulnerable to ocean acidification than suggested by changes in total abundance alone. 
Though experimental investigations have established that coral calcification declines with decreasing saturation $\operatorname{state}^{3,4}(\Omega)$ associated with elevated $\mathrm{pCO}_{2}$, the sensitivity to $\Omega$ differs substantially both between species and within species, limiting our ability to predict how coral communities will respond to ocean acidification. Differences in energy availability for calcification have been suggested to be one reason for differences in the coral calcification response to ocean acidification ${ }^{3}$. Here we show that spawning female corals are more sensitive to ocean acidification than males or non-spawning females. This suggests that the energetically-costly process of egg production may cause gender-linked differences in coral sensitivity to ocean acidification.

Eight colonies of the facultatively symbiotic, gonochoric coral Astrangia poculata were collected from the Woods Hole Oceanographic Institution pier (Woods Hole, MA USA). Fragments from each parent colony, 72 in total, were assigned to 8 flow-through aquaria. During the first month, temperatures were changed gradually from ambient to two treatment values $\left(16^{\circ} \mathrm{C}\right.$ or $24^{\circ} \mathrm{C}, 4$ aquaria per temperature $)$. Corals were held at these temperatures and ambient $\mathrm{pCO}_{2}$ for 9 months (initial phase), after which $\mathrm{CO}_{2}$ levels for two aquaria per temperature were increased by $\sim 400 \mathrm{ppmv}$ (treatment phase). At $24^{\circ} \mathrm{C}$, corals spawned repeatedly, allowing identification of colony gender (egg vs. sperm release). Specimens from a given parent colony spawned as the same gender and gender did not change over time. Gender assignments for $16^{\circ} \mathrm{C}$ aquaria are based on the gamete type released by fragments from the same parent colony grown at $24^{\circ} \mathrm{C}$, since no 
spawning occurred at $16^{\circ} \mathrm{C}$. Calcification rates were estimated via buoyant weighing. Detailed methods are provided online, water chemistry as supplementary materials. The impact of elevated $\mathrm{pCO}_{2}$ on calcification was similar for both male and female corals at $16^{\circ} \mathrm{C}$ (Fig. 1). At $24^{\circ} \mathrm{C}$, however, the impact was significantly different between genders: female corals had significantly reduced calcification rates whereas male corals were not significantly affected by elevated $\mathrm{pCO}_{2}$.

Greater female sensitivity to elevated $\mathrm{pCO}_{2}$ appears to be linked to spawning, since spawning corals $\left(24^{\circ} \mathrm{C}\right)$ showed a pronounced gender difference, while non-spawning corals $\left(16^{\circ} \mathrm{C}\right)$ did not. This gender effect on the calcification response to $\mathrm{CO}_{2}$ could reflect greater energy investment in egg production relative to sperm production. A significant portion of a coral's energy is devoted to reproduction ${ }^{3}$; in females of some coral species, reproduction is delayed until a larger colony size is reached ${ }^{4,5}$, suggesting a trade off between reproduction and growth. If spawning females have less energy available for calcification, they may be more sensitive to ocean acidification. Reduced calcification rates could make female corals more vulnerable to damage and reduce their ability to re-grow. Over time, environmental impacts could lead to a shift in gender ratios, as suggested by high male:female ratios on polluted reefs ${ }^{6}$. A decline in the proportion of female corals within a population could compromise sexual reproduction, making corals more vulnerable to ocean acidification than suggested by changes in community calcification alone. 


\section{References and notes}

1. J. A. Kleypas et al., Science 284, 118 (1999).

2. O. Hoegh-Guldberg et al., Science 318, 1737 (2007).

3. S. Leuzinger, K. R. N. Anthony, B. L. Willis, Oecologia (Berl) 136, 524 (2003).

4. Y. Benayahu, Y. Loya, Biol. Bull. 170, 32 (1986).

5. E. A. Chornesky, E. C. Peters, Biol. Bull 172, 161 (1987).

6. P. L. Harrison, C. C. Wallace, in Coral Reefs Z. Dubinsky Ed. (Elsevier, Amsterdam, 1990) pp.133-207.

7. This work benefited from the assistance of Nobumichi Shimizu, Dave Wellwood, Paul Henderson, Scott Gallager, Ann Tarrant, Jason Smith, Amber York, Fred Keller, Kevin Thompson, Michael Brosnahan, Nan Trowbridge, and Terry Rioux. This material is based upon work supported under a National Science Foundation Graduate Research Fellowship, the WHOI Ocean Life Institute, NSF OCE-0648157, and an International Society for Reef Studies / Ocean Conservancy Fellowship. 

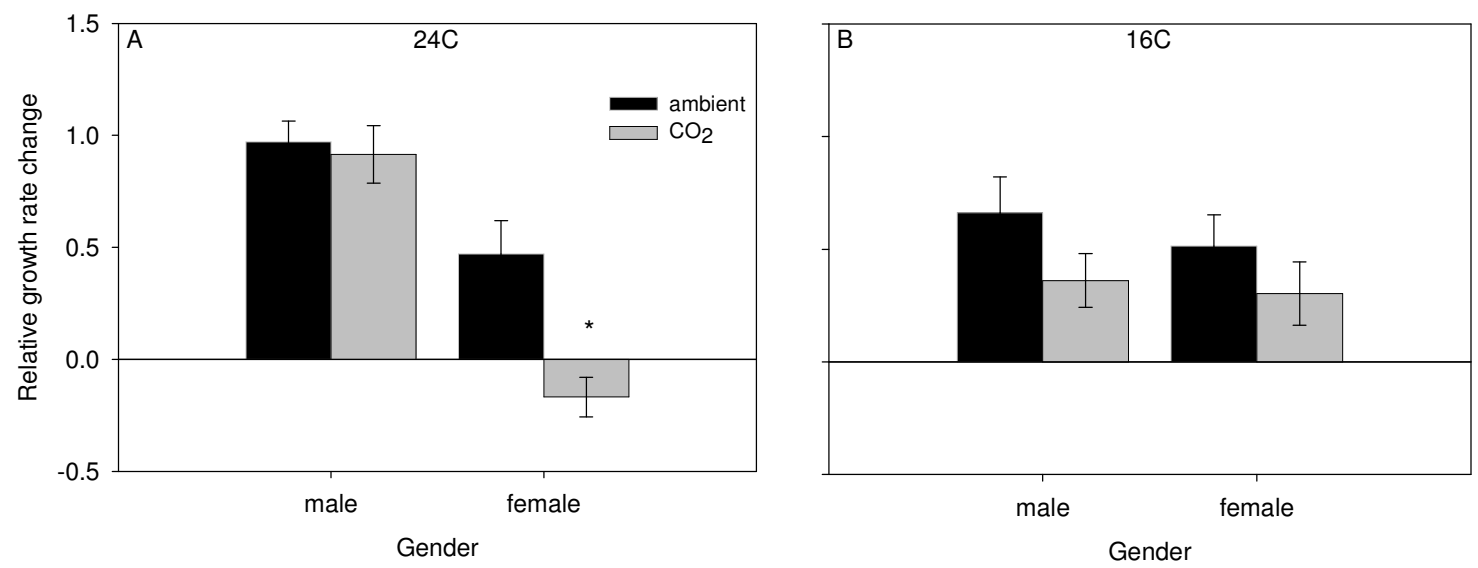

Figure 1. Relative change in growth rate (treatment growth rate minus initial growth rate divided by initial rate) for corals grown at $24^{\circ} \mathrm{C}(\mathrm{A})$ and $16^{\circ} \mathrm{C}(\mathrm{B})$. Corals were reared under ambient $\mathrm{pCO}_{2}$ (black bars), or elevated $\mathrm{pCO}_{2}$ (grey bars) during the treatment phase. Bars represent means, error bars are standard error. Significant differences $(\mathrm{p}<0.01)$ between ambient and $\mathrm{CO}_{2}$ treatments for each gender are indicated with an asterisk. 
Appendix 6: Supplemental materials for Nature paper

\section{Methods}

Coral collection

Four azooxanthellate (white) and four zooxanthellate (brown) colonies of Astrangia poculata were collected September 2008 at a depth of 3-6 m from beneath the Woods Hole Oceanographic Institution pier (Woods Hole, MA USA). Corals were fragmented into 9 pieces and bored regions removed. Each fragment was weighed and attached to individual $\sim 2.5 \mathrm{~cm}$ by $\sim 5 \mathrm{~cm}$ acrylic slides with cyanoacrylate adhesive (EZ

bond). The cyanoacrylate cured for 1day before fragments were re-weighed and assigned to individual aquariums. At least one fragment from each parent colony was assigned to each aquarium.

Aquarium set up

Coral fragments were maintained in a flow through aquarium system provided with $20 \mu \mathrm{m}$ filtered seawater. Water fed into gas mixing chambers to equilibrate the water with a $\mathrm{CO}_{2}$ /air mixture, and from there into valve manifolds used to deliver water to each aquaria. Prior to entering each aquarium, water first passed through a coil of tubing held within the water bath holding the aquaria to allow for temperature equilibration, then entered a small gas mixing chamber held within the water bath to allow for $\mathrm{CO}_{2}$ equilibration at the experimental temperature.

Individual aquaria consisted of $43 \times 5.5 \mathrm{~cm}$ PETG containers filled to $8 \mathrm{~cm}$ depth. Water inlets and outlets were placed at each end of the container and the flow direction altered every $\sim 8 \mathrm{~d}$ such that half the time water entered at the front of the tank and exited 
at the back, while the remainder of the time water entered at the back and exited at the front. Air inlets supplied air to aquarium air-stones placed at the front, middle, and back of each aquarium.

Aquaria were held in water baths with heat controlled by an Omega CN1504 controller using aquarium heaters and thermistor temperature probes, thermo-electric chillers (Ice-probe, Coolworks) cooled the $24^{\circ} \mathrm{C}$ temperature bath, and a compressor based chiller (Prime, Current USA) cooled the $16^{\circ} \mathrm{C}$ bath. Lighting was provided by two T5-HO bulbs (2 10000K bulbs, 48" 54w), on a 12hr 1/d cycle; PAR levels ranged from 0$90 \mu \mathrm{mol}$ photons $/ \mathrm{m}^{2} / \mathrm{s}$.

\section{Feeding}

Corals were fed daily with freshly hatched brine shrimp. A total of $6 \mathrm{ml}$ of shrimp suspension was added to the $16^{\circ} \mathrm{C}$ aquaria, $9 \mathrm{ml}$ to the $24^{\circ} \mathrm{C}$ aquaria. Periodically samples of shrimp suspension were taken, filtered through $0.7 \mu \mathrm{m}$ filter, and dried at $40^{\circ} \mathrm{C}$. The average dry weight of shrimp suspension was $8 \mathrm{mg} / \mathrm{ml}$ (standard deviation: $3 \mathrm{mg}, \mathrm{N}=15$ ).

$\mathrm{CO}_{2}$

During the treatment phase of the experiment $\mathrm{CO}_{2}$ levels were increased for one set of gas mixing chambers and their corresponding aquaria using ambient air (provided by a Porter Cable oil free air-compressor) and $\mathrm{CO}_{2}$. Flow rates for $\mathrm{CO}_{2}$ and air were controlled by rotameters (Alborg Instruments), to provide an air/ $\mathrm{CO}_{2}$ mixture with $\mathrm{CO}_{2}$ levels $400 \mathrm{ppm}$ above ambient. A Qubit s151 $\mathrm{CO}_{2}$ analyzer and a commercially 
prepared $\mathrm{CO}_{2}$ standard (Corp Brothers) were used daily to assess the stability of $\mathrm{CO}_{2}$ levels. A dial barometer (Fisher) was used to measure atmospheric pressure at the time of each $\mathrm{CO}_{2}$ measurement. Gas flow rates to all aquaria were $\sim 1 \mathrm{~L} / \mathrm{min}$.

\section{Chemistry}

Water samples for nutrients, alkalinity, $\mathrm{pH}$ and salinity were collected every $\sim 8$ days throughout the experiment. All vials used for water sampling had polypropylene lids with foamed polyethylene liners. Vials and lids were soaked in distilled water, rinsed repeatedly and dried prior to use. Following sampling, tanks were cleaned of algal growth using a credit card to scrape algae from the sides of the tanks and a pipet to remove algae and detritus from the tank, flow directions were switched and rates adjusted if needed.

\section{Nutrients}

Nutrient levels were monitored both within reservoirs and within individual tanks. Nutrient $\left(\mathrm{NO}_{3}{ }^{-}, \mathrm{PO}_{4}{ }^{3-}, \mathrm{Fe}\right)$ levels were elevated in half of the tanks during the treatment phase, however ambient nutrient levels were high during the treatment phase relative to initial, so data were pooled. Each sample was filtered through a syringe filter $(0.45 \mu \mathrm{m})$ into HDPE scintillation vials with foamed polyethylene lid liners (Wheaton) and stored frozen until measured. Samples were analyzed for ammonium, silicate, phosphate, and nitrate/nitrite (expressed as $\mathrm{NO}_{3}$ ) by the WHOI Nutrient Analytical Facility (using a Lachat Instruments QuickChem 8000 flow injection system). 
Alkalinity

Alkalinity samples were taken in scintillation vials (Wheaton). Samples were stored refrigerated for no more than 1 month prior to measurement. Alkalinities were measured via titration with $0.01 \mathrm{~N} \mathrm{HCl}$ containing $40.7 \mathrm{~g} \mathrm{NaCl} / \mathrm{L}$ using a Metrohm Titrando 808 dosimat and 730 Sample Changer controlled by Tiamo software to perform automated normalized gran titrations of $1 \mathrm{ml}$ samples. Certified seawater reference material supplied by the lab of Andrew Dickson (Scripps Institution of Oceanography) was run each time samples were run to determine acid normality.

$p H$

Prior to the treatment phase, $14 \mathrm{ml} \mathrm{pH}$ samples were collected in $15 \mathrm{ml}$ polypropylene conical tubes (Corning) which had previously been washed repeatedly with fresh water and soaked repeatedly with seawater (to leach out acidic compounds in the plastic). Thymol blue (Fisher lot\#990191) was added to each sample (160 or $320 \mu \mathrm{l}$ of a $2.05 \mathrm{mmol} / \mathrm{L}$ solution). Samples were allowed to temperature equilibrate in a water bath prior to transfer to a $1 \mathrm{~cm}$ cuvette for absorbance measurements. Spectra were collected using an Ocean Optics USB4000 spectrophotometer with a blue filtered tungsten light source (Ocean Optics LS-1-LL). For each sample, 150 spectra were averaged, seawater from one of the sampled tanks was used for establishing reference 
absorbance. pH (total scale) was calculated following Zhang and Byrne (1996) including the correction of Delvalls and Dickson (1998).

During the treatment phase, $\mathrm{pH}$ values in the $\mathrm{CO}_{2}$ treatments were too low to be measured with thymol blue, thus m-cresol purple (Acros 199250050, lot A014923001) was used instead. Initial m-cresol purple measurements were made as described above except dye additions were 50 or $100 \mu \mathrm{l}$ of a $9.4 \mathrm{mmol} / \mathrm{L}$ solution, however most were made using a $10 \mathrm{~cm}$ path length flow cell instead of a cuvette. For measurements with the flow cell, samples were collected in pre-cleaned $10 \mathrm{ml}$ polypropylene syringes (Becton Dickson), a small air bubble introduced (for mixing) along with 50 or $100 \mu \mathrm{l}$ of a $0.946 \mathrm{mmol} / \mathrm{L}$ solution of $\mathrm{m}$-cresol purple. Syringes were capped and placed in a constant temperature bath prior to injection into the flow cell. $\mathrm{pH}$ calculations followed DOE (1994).

In addition to spectrophotometric measurements, $\mathrm{pH}$ (NBS scale) measurements were made on day/night cycles using an Orion Ross 8165BNWP electrode calibrated with NBS buffers (Ricca). Daily cycles in $\mathrm{pH}$ were $\sim 0.1$, with lower values at night. Salinity

Salinity was monitored using a conductivity probe (Hach). Conductivity was converted to salinity values using equations given by Fofonoff (1985).

\section{Buoyant Weight}

Buoyant weight measurements were made every 1-2 months. A Sartorius GC803S balance (resolution $0.2 \mathrm{mg}$, reproducibility of standards was better than $5 \mathrm{mg}$ ) 
with weigh-below hook was used to weigh corals. Weights were used to estimate water density. Mass changes are assumed to reflect changes in the mass of aragonite, an aragonite density of $2.9 \mathrm{~g} / \mathrm{cm}^{3}$ was used to calculate the dry weight of calcium carbonate deposited. Buoyant weight measurements were corrected for the contribution of the acrylic slide and cyanoacrylate.

\section{Gender identification}

Genders were established either by direct observation of gamete release, or by verifying the presence of sperm in incubations of individual specimens in which the water had become cloudy. Direct observation of spawning was made when corals were weighed. During most weighing sessions, some corals spawned allowing for the gamete type released by individual polyps to be identified. Spawning males were readily identified as specimens tend to release sperm in several pulses providing several opportunities to observe gamete release. Female identification was difficult as fewer eggs were released, generally in one or two pulses. Only those specimens observed as they were releasing eggs could be identified as female. In individual incubations carried out for alkalinity depletion and oxygen consumption measurements (not discussed), spawning males often released sperm in sufficient quantity to cloud the water and microscopic examination of water samples from individual incubations which had become cloudy allowed for the identification of sperm and establishment of gender. Not all specimens were observed spawning. Gender assignments for fragments which were 
not observed spawning were based on the gamete type released by other fragments from the same parent colony which were observed spawning.

\section{Statistics}

Growth rates were expressed as differences between the treatment and pretreatment rates relative to the pre-treatment rate, differences between treatments were detected using a paired t-test (Zar 1983). 
Table S1. Day-time water chemistry during initial and treatment phases. Average alkalinity ( $\mu \mathrm{mol} / \mathrm{kg}), \mathrm{pH}$ (total scale, value at experimental temperature), aragonite saturation state $\left(\Omega_{\mathrm{Arag}}\right), \mathrm{CO}_{3}{ }^{2-}(\mu \mathrm{mol} / \mathrm{kg}), \mathrm{HCO}_{3}{ }^{-}(\mu \mathrm{mol} / \mathrm{kg}), \mathrm{pCO} 2(\mu$ atm $)$, salinity, $\mathrm{NH}_{4}{ }^{+}, \mathrm{H}_{2} \mathrm{SiO}_{4}, \mathrm{PO}_{4}{ }^{3-}$, and $\mathrm{NO}_{3}{ }^{-}(\mu \mathrm{M})$. Measured alkalinity, temperature, salinity, nutrient, and $\mathrm{pH}_{\mathrm{T}}$ values were used to calculate saturation state and carbon speciation using a Matlab implementation of CO2SYS ${ }^{\mathrm{S} 6, \mathrm{~S} 7, \mathrm{~S} 8, \mathrm{S9}}$ which incorporated the contribution of ammonia ${ }^{\mathrm{S} 10}$. Values are means, standard deviation in parenthesis.

\begin{tabular}{|c|c|c|c|c|c|c|c|c|c|c|c|}
\hline Initial & Alk & $\mathrm{pH}_{\mathrm{T}}$ & $\Omega_{\text {Arag }}$ & $\mathrm{CO}_{3}{ }^{2-}$ & $\mathrm{HCO}_{3}^{-}$ & $\mathrm{pCO}_{2}$ & $\mathrm{~S}$ & $\mathrm{NH}_{4}^{+}$ & $\mathrm{H}_{2} \mathrm{SiO}_{4}$ & $\mathrm{PO}_{4}^{3-}$ & $\mathrm{NO}_{3}$ \\
\hline $24^{\circ} \mathrm{C}$ ambient & $\begin{array}{l}2110 \\
(20)\end{array}$ & $\begin{array}{l}7.99 \\
(0.03)\end{array}$ & $\begin{array}{l}2.5 \\
(0.1)\end{array}$ & $\begin{array}{l}160 \\
(8)\end{array}$ & $\begin{array}{l}1720 \\
(30)\end{array}$ & $\begin{array}{l}440 \\
(40)\end{array}$ & $\begin{array}{l}32 \\
(0.4)\end{array}$ & $\begin{array}{l}0.9 \\
(0.3)\end{array}$ & $\begin{array}{l}3 \\
(2)\end{array}$ & $\begin{array}{l}0.2 \\
(0.3)\end{array}$ & $\begin{array}{l}2 \\
(2)\end{array}$ \\
\hline $24^{\circ} \mathrm{C} \mathrm{CO}_{2}$ & $\begin{array}{l}2120 \\
(20)\end{array}$ & $\begin{array}{l}7.98 \\
(0.03)\end{array}$ & $\begin{array}{l}2.5 \\
(0.1)\end{array}$ & $\begin{array}{l}155 \\
(8)\end{array}$ & $\begin{array}{l}1740 \\
(20)\end{array}$ & $\begin{array}{l}450 \\
(40)\end{array}$ & $\begin{array}{l}32 \\
(0.3)\end{array}$ & $\begin{array}{l}0.7 \\
(0.2)\end{array}$ & $\begin{array}{l}3 \\
(1)\end{array}$ & $\begin{array}{l}0.13 \\
(0.08)\end{array}$ & $\begin{array}{l}1 \\
(0.6)\end{array}$ \\
\hline $16^{\circ} \mathrm{C}$ ambient & $\begin{array}{l}2120 \\
(20)\end{array}$ & $\begin{array}{l}8.01 \\
(0.03)\end{array}$ & $\begin{array}{l}2.1 \\
(0.1)\end{array}$ & $\begin{array}{l}130 \\
(10)\end{array}$ & $\begin{array}{l}1790 \\
(20)\end{array}$ & $\begin{array}{l}420 \\
(30)\end{array}$ & $\begin{array}{l}31 \\
(0.4)\end{array}$ & $\begin{array}{l}0.9 \\
(0.4)\end{array}$ & $\begin{array}{l}3 \\
(1)\end{array}$ & $\begin{array}{l}0.2 \\
(0.1)\end{array}$ & $\begin{array}{l}0.8 \\
(0.5)\end{array}$ \\
\hline $16^{\circ} \mathrm{C} \mathrm{CO}_{2}$ & $\begin{array}{l}2120 \\
(20)\end{array}$ & $\begin{array}{l}8.01 \\
(0.03)\end{array}$ & $\begin{array}{l}2.1 \\
(0.1)\end{array}$ & $\begin{array}{l}130 \\
(10)\end{array}$ & $\begin{array}{l}1790 \\
(20)\end{array}$ & $\begin{array}{l}420 \\
(40)\end{array}$ & $\begin{array}{l}31 \\
(0.4)\end{array}$ & $\begin{array}{l}1 \\
(0.3)\end{array}$ & $\begin{array}{l}3 \\
(2)\end{array}$ & $\begin{array}{l}0.2 \\
(0.1)\end{array}$ & $\begin{array}{l}1 \\
(0.5)\end{array}$ \\
\hline Treatment & & & & & & & & & & & \\
\hline $24^{\circ} \mathrm{C}$ ambient & $\begin{array}{l}2040 \\
(20)\end{array}$ & $\begin{array}{l}8.00 \\
(0.03)\end{array}$ & $\begin{array}{l}2.5 \\
(0.2)\end{array}$ & $\begin{array}{l}160 \\
(10)\end{array}$ & $\begin{array}{l}1650 \\
(30)\end{array}$ & $\begin{array}{l}410 \\
(40)\end{array}$ & $\begin{array}{l}31 \\
(0.6)\end{array}$ & $\begin{array}{l}1 \\
(0.4)\end{array}$ & $\begin{array}{l}5 \\
(1)\end{array}$ & $\begin{array}{l}0.8 \\
(0.2)\end{array}$ & $\begin{array}{l}6 \\
(3)\end{array}$ \\
\hline $24^{\circ} \mathrm{C} \mathrm{CO}_{2}$ & $\begin{array}{l}2050 \\
(20)\end{array}$ & $\begin{array}{l}7.79 \\
(0.04)\end{array}$ & $\begin{array}{l}1.7 \\
(0.1)\end{array}$ & $\begin{array}{l}110 \\
(10)\end{array}$ & $\begin{array}{l}1790 \\
(30)\end{array}$ & $\begin{array}{l}720 \\
(70)\end{array}$ & $\begin{array}{l}31 \\
(0.6)\end{array}$ & $\begin{array}{l}1 \\
(0.3)\end{array}$ & $\begin{array}{l}5.5 \\
(0.6)\end{array}$ & $\begin{array}{l}0.7 \\
(0.2)\end{array}$ & $\begin{array}{l}6 \\
(3)\end{array}$ \\
\hline $16^{\circ} \mathrm{C}$ ambient & $\begin{array}{l}2030 \\
(40)\end{array}$ & $\begin{array}{l}8.02 \\
(0.04)\end{array}$ & $\begin{array}{l}2.0 \\
(0.2)\end{array}$ & $\begin{array}{l}130 \\
(10)\end{array}$ & $\begin{array}{l}1710 \\
(30)\end{array}$ & $\begin{array}{l}390 \\
(30)\end{array}$ & $\begin{array}{l}31 \\
(0.2)\end{array}$ & $\begin{array}{l}2 \\
(1.5)\end{array}$ & $\begin{array}{l}3 \\
(1)\end{array}$ & $\begin{array}{l}1 \\
(0.4)\end{array}$ & $\begin{array}{l}6 \\
(4)\end{array}$ \\
\hline $16^{\circ} \mathrm{C} \mathrm{CO}_{2}$ & $\begin{array}{l}2040 \\
(30)\end{array}$ & $\begin{array}{l}7.75 \\
(0.04)\end{array}$ & $\begin{array}{l}1.2 \\
(0.1)\end{array}$ & $\begin{array}{l}75 \\
(6)\end{array}$ & $\begin{array}{l}1850 \\
(30)\end{array}$ & $\begin{array}{l}790 \\
(70)\end{array}$ & $\begin{array}{l}30.7 \\
(0.2)\end{array}$ & $\begin{array}{l}1.5 \\
(0.5)\end{array}$ & $\begin{array}{l}4 \\
(1)\end{array}$ & $\begin{array}{l}0.9 \\
(0.3)\end{array}$ & $\begin{array}{l}6 \\
(3)\end{array}$ \\
\hline
\end{tabular}


References

S1 H. Zhang, R. H. Byrne, Mar. Chem. 52, 17 (1996).

S2 T. A. Delvalls, A. G. Dickson, Deep-Sea Res 45, 1541 (1998).

S3 DOE, A. G. Dickson, C. Goyet, Eds. ORNL/CDIAC-74 (1994).

S4 N. Fofonoff, J. Geophys. Res. 90, 3332 (1985).

S5 J.H. Zar. Biostatistical Analysis (Prentice Hall, Englewood Cliffs, NJ 1984)

S6 van Heuven, S., D. Pierrot, E. Lewis, D. W. R. Wallace, Carbon Dioxide Information

Analysis Center, Oak Ridge National Laboratory, U.S. Department of Energy, Oak

Ridge, Tennessee (2009)

S7 C. Mehrbach, C. H. Culberson, J. E. Hawley, R. M. Pytkowicz, Limnol.

Oceanography 18, 897 (1973).

S8 A. G. Dickson, F. J. Millero. Deep-Sea Res. 34:1733 (1987).

S9 A. G. Dickson, Deep-Sea Res 37, 755 (1990).

S10 S. L. Clegg, M. Whitfield, Geochim Cosmochim Acta 59, 2403 (1995). 